\title{
Combination therapy in rheumatoid arthritis
}

Citation for published version (APA):

Verhoeven, A. C. (2002). Combination therapy in rheumatoid arthritis. [Doctoral Thesis, Maastricht University]. Universiteit Maastricht. https://doi.org/10.26481/dis.20020322av

Document status and date:

Published: 01/01/2002

DOI:

10.26481/dis.20020322av

Document Version:

Publisher's PDF, also known as Version of record

\section{Please check the document version of this publication:}

- A submitted manuscript is the version of the article upon submission and before peer-review. There can be important differences between the submitted version and the official published version of record.

People interested in the research are advised to contact the author for the final version of the publication, or visit the DOI to the publisher's website.

- The final author version and the galley proof are versions of the publication after peer review.

- The final published version features the final layout of the paper including the volume, issue and page numbers.

Link to publication

\footnotetext{
General rights rights.

- You may freely distribute the URL identifying the publication in the public portal. please follow below link for the End User Agreement:

www.umlib.nl/taverne-license

Take down policy

If you believe that this document breaches copyright please contact us at:

repository@maastrichtuniversity.nl

providing details and we will investigate your claim.
}

Copyright and moral rights for the publications made accessible in the public portal are retained by the authors and/or other copyright owners and it is a condition of accessing publications that users recognise and abide by the legal requirements associated with these

- Users may download and print one copy of any publication from the public portal for the purpose of private study or research.

- You may not further distribute the material or use it for any profit-making activity or commercial gain

If the publication is distributed under the terms of Article $25 \mathrm{fa}$ of the Dutch Copyright Act, indicated by the "Taverne" license above, 


\section{Combination therapy in rheumatoid arthritis}

A.C. Verhoeven 
The randomised controlled trial (COBRA; combinatietherapie bij vroege reumatoïde artritis) presented in this thesis was funded by grant $92-045$, Ontwikkelingsgeneeskunde, Ziekenfondsraad, Amstelveen, The Netherlands.

Patients, rheumatologists and research personnel from the Rheumatology Departments of the following clinical centres participated in the COBRA trial: the University Hospitals of Maastricht, Leiden, and Leuven (Belgium); Daniël den Hoed Kliniek, Rotterdam; Medisch Spectrum Twente en Twenteborg, Enschede and Almelo; Jan van Breemen Instituut, Amsterdam; Bronovo Hospital, The Hague; Reinier de Graaf Hospital, Delft; Laurentius Hospital, Roermond; Medical Centre Alkmaar. The coordinating centre and pharmacy were in the Maastricht University Hospital.

Verhoeven, A.C. $\odot 2002$

Combination therapy in rheumatoid arthritis

Ph.D. thesis, University Maastricht.

ISBN 90-9015585-6

Cover: La piramide de Kukulkan, Chichén Itzá; John C. Mureiko @, USA, 1996.

Illustrations by Corneille, and Babette Rosman. Production: Print Partners Ipskamp, Enschede. Financial support by the Reumafonds is gratefully acknowledged. 


\section{Combination therapy in rheumatoid arthritis}

\section{PROEFSCHRIFT}

ter verkrijging van de graad van doctor aan de Universiteit Maastricht

op gezag van de Rector Magnificus

Prof. dr A.C. Nieuwenhuijzen Kruseman, volgens het besluit van het College van Decanen in het openbaar te verdedigen op vrijdag, 22 maart 2002 om 12 uur

door

Adrianus Comelis Leonardus Paulus Josef Verhoeven 


\section{Promotores}

Prof. dr J.MJ.P. van der Linden

Prof. dr M. Boers (Vrije Universiteit Medisch Centrum, Amsterdam)

\section{Beoordelingscommissie}

Prof. dr ir P.A. van den Brandt (voorzitter)

Prof. dr J.M.A.van Engelshoven

Prof. dr HJ.M. Hazes (Erasmus MC, Rotterdam)

Dr J.L. Severens 
Sun come up it was blue and gold Sun come up it was blue and gold Sun come up it was blue and gold ever since I put your picture

in a frame

I come calling in my Sunday best I come calling in my Sunday best I come calling in my Sunday best ever since I put your picture in a frame

I'm gonna love you till the wheels come off oh yea

I love you baby and I always will I love you baby and I always will I love you baby and I always will ever since I put your picture

in a frame

Tom Waits / Kathleen Brennan from Mule Variations ㄷ 1999 



\section{CONTENTS}

1 Combination therapy in rheumatoid arthritis: introduction to thesis

2 Combination therapy in rheumatoid arthritis: up-dated systematic review

3 Randomised comparison of combined step-down prednisolone, methotrexate and sulphasalazine with sulphasalazine alone in early rheumatoid arthritis

4 Cost-effectiveness and cost-utility of combination therapy in early rheumatoid arthritis: randomised comparison of combined step-down prednisolone, methotrexate and sulphasalazine with sulphasalazine alone

5 Limited bone loss due to corticosteroids; a systematic review of prospective studies in rheumatoid arthritis and other diseases

6 Reliability of spot samples for assessment of urinary excretion of pyridinoline in patients with rheumatoid arthritis

7 Bone turnover, joint-damage and bone mineral density in early rheumatoid arthritis treated with combination therapy including high-dose corticosteroids

8 Factors predicting response to treatment in rheumatoid arthritis: the importance of disease duration

9 Responsiveness of the core-set, response criteria and utility measures in rheumatoid arthritis

10 American College of Rheumatology criteria for improvement in rheumatoid arthritis should only be calculated from scores that decrease on improvement

11 Validity of the MACTAR questionnaire as functional index in a rheumatoid arthritis clinical trial

12 Summary \& General discussion 
Samenvatting

Dankwoord

Publicaties

190

Curriculum vitae 


\section{CHAPTER 1}

\section{INTRODUCTION TO THIS THESIS}

Arco Verhoeven 


\section{Rheumatoid arthritis}

RHEUMATOID ARTHRTTS (RA) is a disease of unknown aetiology, characterised by inflammation of multiple joints and systemic manifestations [1]. The inflammation is primarily located in the synovia and tendon sheaths of peripheral joints of hands, wrists and feet, and is usually symmetrical. RA is a common disease with prevalence between 1 and $1.5 \%$ in people above 40 years of age [2]. The peak incidence is in the fifth decade and women are affected approximately twice as often as men are. Where in a minority of RA patients the disease has a relatively mild course with non-erosive disease, the majority has an exacerbating and remitting arthritis [3]. In these patients, RA usually leads to progressive disability due to destruction of joints and surrounding structures [4-9]. Structural and irreversible damage to the joints is radiographically demonstrated early in the course of the disease $[10,11]$. Progression of joint damage is associated with disease activity and most prominent during the first years after onset $[11,12]$. The disease and its therapy can cause organ dysfunction. There is an excess mortality among patients with RA; estimated life expectancies are 3 to 10 years less than persons without RA [11-15]. To date, the therapy is palliative and directed at decreasing symptoms, slowing joint destruction and optimising physical function [16].

\section{The treatment of RA}

Traditionally the treatment comprised rest, life style rules, and non-steroidal anti-inflammatory drugs (NSAIDs, such as ibuprofen). NSAIDs relieve symptoms such as pain and stiffness, but do not retard long-term joint damage and physical disability [17].

Patients who show none or insufficient response to the initial therapy receive - after some time that is needed for evaluation of the treatment response - another pharmaceutical drug; a socalled disease-modifying anti-rheumatic drug (DMARD). Unlike NSAIDs, these types of drugs do have the potential to retard long-term joint damage and associated physical disability. Examples of DMARDS are anti-malarials, gold salts, sulphasalazine, methotrexate, leflunomide, cyclosporin, cyclophosphamide, and penicillamine. There is extensive documentation of their clinical effectivity as well as their toxicity [18-25]. Moreover, evidence is accumulating that many of these (for example, sulphasalazine and methotrexate) slow radiographic progression in RA [26,27]. Felson and co-workers showed in two meta-analyses that of the individual second-line drugs methotrexate and sulphasalazine had the best efficacy/toxicity profiles [28,29]. Not all of these data were available at the time in which the COBRA trial described in this thesis was designed; nevertheless some indications already existed to favour sulphasalazine and methotrexate as effective agents with relatively quick onset (compared with traditional "first choice" intramuscular gold salts) [30]. In fact, it seems that in Europe a situation has developed in which sulphasalazine is DMARD of first choice whereas in the New World this is methotrexate [31].

DMARDs are also called SAARDs [32]; slow-acting anti-rheumatic drugs as it may take several months before the beneficial effects appear. Various adverse drug effects may occur and give reason to switch to treatment with another DMARD [33]. A part of the patients that started DMARD therapy will show insufficient response, and this also may give reason to switch to another DMARD. Most properly this is a DMARD with a putatively more effective but also more 
toxic profile. Thus patient groups with insufficient treatment response or adverse drug events climb the steps of the so-called 'therapeutic pyramid' [34]. As time passes without adequate control of disease activity the consequences in terms of joint damage and disability accumulate.

During the last decades there was a call for earlier and more aggressive intervention directed at suppression of the disease's activity; the inflammation of the synovia as well as the systemic effects; some even speak of shift of paradigm [35-40]. A more aggressive approach may be treatment with DMARDs earlier in the course of the disease, using combinations of DMARDs, or treatment with corticosteroids early in the course of the disease [41,42].

\section{Combination therapy}

Combining anti-rheumatic drugs has theoretical appeal as it could result in more efficacy when the mechanism of action of the drugs is different and possibly less toxic when the combined drugs can be given at lower dose [43]. Interest in combination therapy was evident as early as 1951, when means were sought to maintain the remission induced with corticosteroids at a lower dose of these drugs [44]. Treatment with drug combinations appears quite common as revealed by several surveys [45]. There are many ways to combine drugs; most frequently, a second drug is added when the patient has insufficient response to the first. This can be seen as a variation of the classical step by step approach in which a patient 'climbs the pyramid' of treatment with ever more toxic and putatively more effective drugs. According to the call to 'remodel the pyramid', patients with early RA can also be treated with multiple drugs to suppress activity before the irreversible joint damage occurs. This means aggressive as well as early treatment. Alongside the COBRA study described in this thesis [46], some empirical evidence has come available to support early intervention with second-line anti-rheumatic drugs [47]. However, results from these and other studies in terms of prevention of radiographic evident joint damage are modest.

Critical appraisal of previous studies that evaluated the advantage of anti-rheumatic drug combinations over single drug treatment concluded that such advantage remained to be proven as many of the trials hampered by insufficient quality of design and reporting [48]. Notably, a metaanalysis of all kind of investigated combinations taken together failed to demonstrate efficacy of combination therapy [49].

\section{Corticosteroids}

Corticosteroids are divided in glucocorticoids and mineralocorticoids. Both are hormones produced by the adrenal cortex with broad anti-inflammatory and immunosuppressive effects of which the underlying mechanisms are incompletely understood. Glucocorticoids are preferred to mineralocorticoids for their smaller mineralocorticoid effect. Prednisone and prednisolone are glucocorticoids that are widely employed for treatment of RA, but mostly in established disease [34]. Despite dramatic short-term effects, physicians and patients became disillusioned soon after the introduction of this medication during the fifties when the long-term side effects became apparent [50-52]. Since then corticosteroids are widely regarded as agents of last resort, with nearly unacceptable benefit-to-harm ratio [34,53]. Although the evidence to the contrary is building up [54], the dogma holds that corticosteroids' action is merely symptomatic and not diseasemodifying [53]. Evidence from RA as well as other diseases treated with corticosteroids suggests 
that long-term treatment with $7.5 \mathrm{mg} /$ day or less is associated with low toxicity [55-57]. Saag et al concluded from a meta-analysis summarising data from sparsely available controlled studies, that corticosteroids appear to be approximately as effective as the traditional second-line antirheumatic drugs [58]. The therapeutic value of corticosteroids in addition or synergy with (other) second-line drugs remains uncertain. There is great concern about adverse effects that corticosteroids can bring. Obviously, the well-known and extensively reviewed adverse effects associated with long-term and supraphysiological doses of corticosteroids should never be neglected. They include suppression of the hypothalamic axis, increased susceptibility to infection, gastrointestinal ulceration and perforation (due to combination with NSAIDs), osteopenia and osteoporosis, aseptic necrosis of bone, skin changes, myopathia, psychosis, cataract, glaucoma, fluid retention, hypertension, enhanced atherosclerosis, and insulin resistance. Controversy remains around prevalence, magnitude, clinical relevance and minimum safe dose with almost every glucocorticoid induced side effect. Attention is often focused at osteoporosis, as this is a highly prevalent disorder in the general population of elderly. Furthermore, also RA itself is associated with bone loss through functional impairment and active disease [59].

THIS THESIS contains three parts with chapters that all correspond to papers that are published [46, 60-68]. THE FIRST PART - with chapters 2,3 , and 4 - respectively, describes a systematic review on combination therapy, and two reports from the randomized controlled trial named COBRA after the Dutch acronym "combinatietherapie bij vroege reumatoïde artritis" (combination therapy in early rheumatoid arthritis).

The systematic review in chapter 2 [60] fits in a sequence of systematic reviews; it is an update of earlier publications $[69,70]$. The flow of new studies on combination therapy in RA gave reason and opportunity to re-investigate the potential efficacy and synergy of various combinations.

Chapter 3 [45] describes the randomised clinical trial performed to compare the clinical outcomes of combined step-down prednisolone, methotrexate and sulphasalazine with sulphasalazine alone in patients with early and active RA. The purpose of this large, and state-ofthe-art study was to clarify the till that point in time unproven value of intensive combination therapy in early RA. The main outcomes were a pooled index (weighted change score of five disease activity measures) [71] and Sharp/van der Heijde radiographic damage score in hands and feet $[72,73]$. Independent health-care professionals assessed the main outcomes without knowledge of treatment allocations.

Monetary costs of RA are high due to increased use of medical services (such as medication and hospitalisation), and work disability developing during the course of the disease $[8,9,74,75]$. Chapter 4 [61] evaluates the cost-effectiveness and cost-utility properties of the combination therapy described in chapter 3 . The study comprised full economic analysis of all direct costs, and also assessed patient-derived utilities (quality of life) by rating scale and standard gamble measurement techniques [76]. These have a theoretical advantage of allowing comparisons across different diseases [77,78]. 
THE SECOND PART OF THIS THESIS - with chapters 5, 6, and 7 - focuses at deleterious effects of corticosteroids in RA. Adverse effects of corticosteroids especially on bony tissues are the topic of -justified- concern. In chapter 5 [62] corticosteroid induced bone loss is reviewed. Initially, we sought for a quantification (dose-response relation) on the basis of data derived from randomised controlled trials in this field. When it appeared that there were only two publications of such studies (both with RA patients) [79,80], we broadened our scope to all prospective research that was adequately performed and documented, i.e. all reports with reliable and serial bone density measurements. Thus, we were able to shed some light on the relation between changes in bone density, the disease treated, and the prescribed dose of corticosteroids.

In RA, there is inflammation at the site of joints, associated with typical juxtaarticular osteoporosis. Apart from this, there is general bone loss associated with disease activity [59]. As a result of pathologic destruction of collagen in bone and cartilage, crosslinks between mature collagen fibers are resorbed more rapidly than normal. This causes a rise in circulating collagen crosslink levels and their urinary excretion [81]. The concise report of the study described in chapter 6 [63] was initiated to determine how well a urinary spot sample of patients with active RA can predict 24-hours urinary excretion of collagen crosslinks (pyridinoline and deoxypyridinoline).

Chapter 7 [64] is focused on changes in bone metabolism at different levels of disease activity, both with and without oral corticosteroid therapy. The data were derived from the COBRA study described in chapter 3; alongside with assessment of clinical measures, radiographic outcomes and bone density, were samples of serum and urine stored for investigation.

THE LAST PART OF THIS THESIS - with chapters 8, 9, 10, and 11 - is on assessment of outcomes in rheumatoid clinical trials. This is particularly difficult, as there are so many measures of such wide variety to assess treatment efficacy in RA in and outside the context of a clinical trial [82-84]. The OMERACT consensus on endpoint measures in RA facilitates comparison of results from different trials in treatment evaluation $[85,86]$. Many have called for further validation of the measures included in the core set adopted by the World Health Organization/ International League of Associations for Rheumatology (WHO/ILAR) and American College of Rheumatology (ACR) [87-90]. This WHO/ILAR core set to be used as a minimum in every RA clinical trial, comprises an acute phase reactant, disability, pain, patient's and physician's global assessment, swollen and tender joint counts, and radiographic studies of joints in any trial with a follow-up of 1 year or longer [87].

An important factor in the benefits that may be achieved with pharmaceutical treatment is the duration of the disease. Seemingly, the odds for prevention of damage are better in the earlier phases of the disease. On the other hand it remains fairly unknown why some patients with RA respond better to treatment than others. It has been suggested that patients with more severe disease may be less likely to show a response, and that patients who have previously been treated with second-line drugs also may have more recalcitrant disease [16]. In addition, there are indications that the biologic process of RA changes early in the disease, so that patients may be less responsive to treatment over time $[47,91,92]$. It would also appear that patients with especially active disease are more likely to respond to treatment, particularly in the context of a clinical trial. 
Better response in these patients would then - partially - represent regression to the mean. Our understanding of which types of patients with RA are most likely to respond to treatment has implications for the aggressiveness of treatment of individual patients. In addition, there may be implications for the design of clinical trials, in that the anticipated power of a trial may be a function of the particular mix of patients that participate in the trial. To address these questions patient data from 14 diverse clinical trials of second-line drugs and devices in RA conducted since the early 1980s have been used. The COBRA trial described chapter 3 was one of these trials and the one with the earliest intervention [46]. The analysis is presented in chapter 8 [65].

Chapter $9[66]$ fits in the process of the ongoing validation of the responsiveness of the core set, of the ACR improvement and the European League Against Rheumatism (EULAR) response criteria $[93,94]$, and of other single measures and indices. Again, we used data from the COBRA trial that allowed us to create one "gold" and one "silver" standard for relevant response against which to validate performance of end point measures: we proposed the hypothesis a priori that patients on combined treatment would on average show large and, certainly, relevant improvements at week 16 of follow-up owing to the corticosteroid pulse (gold standard). Also, we assumed on the basis of the well known effects of sulphasalazine on disease activity that patients in the sulphasalazine-only group would also show relevant improvements, but to a lesser degree (silver standard). Subsequently, we ranked all comprised end point measures and indices by their relative responsisveness, and also by their ability to discriminate between treatment groups.

The measurement of relative changes in some of the core set outcome measures made us aware of unforeseen piffalls that urged us to formulate explicit recommendations as to the calculation of relative changes in chapter 10 [67].

Physical function is an important outcome in RA and included in the WHO/ILAR core set for assessment of this disease's outcomes [87]. Examples of widely used questionnaires to measure physical function are the Stanford Health Assessment Questionnaire (HAQ) and Arthritis Impact Measurement Scale (AIMS) $[95,96]$. However, these measures do not include patient priorities. Including such priorities may lead to better face validity and responsiveness. An example of a functional ability scale that takes account of patient priorities is the McMaster Toronto Arthritis Patient Preference Disability Questionnaire (MACTAR) [97]. Its developers noted good responsiveness in RA patients in a controlled trial that demonstrated clinically important change [97]. In the MACTAR, the interviewer elicits which 5 impaired activities are most important to an individual patient, and follows their changes over time. The MACTAR also includes questions on social and emotional functioning and probes whether any problems are due to arthritis. Because of this format, the MACTAR concept of function may be more comprehensive than that of traditional fixed-item questionnaires such as the HAQ, and reveal issues that really matter to the patient. Chapter 11 [68] presents a critical evaluation of the feasibility and validity of this instrument [98]. The data came from the same randomised trial of combined treatment in early RA patients, described in detail in chapter 3 (COBRA study). 


\section{References}

1. Harris ED Jr. Rheumatoid arthritis. Pathophysiology and implications for therapy. N Engl J Med 1990; 322:1277-89.

2. Dieppe P, Klippel JH Rheumatic diseases, Rheumatology. London, Mosby, 1994.

3. Pinals RS, Masi AT, Larsen RA, and the Subcommittee for Criteria of Remission in Rheumatoid Arthritis of the American Rheumatism Association Diagnostic and Therapeutic Criteria Committee: Preliminary criteria for remission in rheumatoid arthritis. Arthritis Rheum 1981; 24:1308-1315.

4. Brook A, Corbett M. Radiographic changes in early RA. Ann Rheum Dis 1977; 36:71-73.

5. Pincus T, Callahan LF, Sale WG, Brooks AL, Payne LE, Vaughn WK. Severe functional declines, work disability, and increased mortality in seventy-five rheumatoid arthritis patients studied over nine years. Arthritis Rheum 1984; 27:864-72.

6. Scott DL, Symmons DPM, Coulton BL, Popert AJ. Long-term outcome of treating rheumatoid arthritis: Results after 20 years. Lancet 1987; i:1108-11.

7. Harrison BJ, Symmons DP, Brennan CR, Barrett EM, Scott DG, Silman AJ. Inflammatory arthritis in the community is not a benign disease: predicting functional disability one year after presentation. J Rheumatol 1996; 23: 1326-31.

8. Yelin E, Meenan R, Nevitt M, Epstein W. Work disability in rheumatoid arthritis: effects of disease, social, and work factors. Ann Intern Med 1980; 93:551-6.

9. Jäntti J, Ahol K, Kaarela K, Kautiainen $\mathrm{H}$. Work disability in an inception cohort of patients with seropositive rheumatoid arthritis: a 20 year study, Rheumatology 1999; 38: 1138-41.

10. Fuchs HA, Kaye JJ, Callahan LF, Nance EP, Pincus T. Evidence of significant radiographic damage in rheumatoid arthritis within the first 2 years of disease. J Rheumatol 1989; 16:585-9.

11. van der Heijde DMFM, van Leeuwen MA, van Riel PLCM, Koster AM, van ' $t$ Hof MA, Rijswijk MH, van de Putte LBA. Biannual radiographic assessments of hands and feet in a three-year prospective followup of patients with early rheumatoid arthritis. Arthritis Rheum 1992; 35:26-34.

12. Möttönen TT. Prediction of erosiveness and rate of development of new erosions in early rheumatoid arthritis. Ann Rheum Dis 1988; 47:648-53.

13. Rasker JJ, Cosh JA. Cause and age at death in a prospective study of 100 patients with rheumatoid arthritis. Ann Rheum Dis 1981; 40:115-20.

14. Wolfe F, Mitchell DM, Sibley JT, et al. The mortality in rheumatoid arthritis. Arthritis Rheum 1994; 37:481-94.

15. Gabriel SE, Crowson CS, OFallon WM. Mortality in rheumatoid arthritis: Have we made an impact in 4 decades? J Rheumatol 1999; 26:2529-33.

16. American College of Rheumatology Ad Hoc Committee on Clinical Guidelines. Guidelines for the management of rheumatoid arthritis. Arthritis Rheum 1996; 39: 713-722.

17. Cash JM, Klippel JH. Second-line drug therapy for rheumatoid arthritis. N Engl J Med 1994; 330:1368-1375.

18. Suarez-Almazor ME, Belseck E, Shea B, Homik J, Wells G, Tugwell P. Antimalarials for treating rheumatoid arthritis. Cochrane Database Syst Rev 2000; 4: CD000959.

19. Cark P, Tugwell P, Bennet K, Bombardier C, Shea B, Wells G, Suarez-Almazor ME. Injectable gold for rheumatoid arthritis. Cochrane Database Syst Rev 2000; 2: CD000520.

20. Suarez-Almazor ME, Belseck E, Shea B, Wells G, Tugwell P. Sulfasalazine for theumatoid arthritis. Cochrane Database Syst Rev 2000; 2: CD000958.

21. Suarez-Almazor ME, Belseck E, Shea B, Wells G, Tugwell P. Methotrexate for rheumatoid arthritis. Cochrane Database Syst Rev 2000; 2: CD000957. 
22. Strand V, Cohen S, Schiff M, Weaver A, Fleischmann R, Cannon G, Fox R, Moreland L, Olsen N, Furst D, Caldwell J, Kaine J, Sharp J, Hurley F, Loew-Friedrich I. Treatment of active heumatoid arthritis with leflunomide compared with placebo and methotrexate. Leflunomide Rheumatoid Arthritis Investigators Group. Arch Intern Med 1999; 159:2542-50.

23. Wells G, Haguenauer D, Shea B, Suarez-Almazor ME, Welch VA, Tugwell P. Cyclosporin for rheumatoid arthritis. Cochrane Database Syst Rev 2000; 2: CD001083.

24. Suarez-Almazor ME, Belseck E, Shea B, Wells G, Tugwell P. Cyclophosphamide for treating rheumatoid arthritis. Cochrane Database Syst Rev 2000; 4: CD001157.

25. Suarez-Almazor ME, Spooner C, Belseck E. Penicillamine for treating rheumatoid arthritis. Cochrane Database Syst Rev 2000; 4: CD001460.

26. Lopez-Mendez A, Daniel WW, Reading JC, Ward JR, Alarcon GS. Radiographic assessment of disease progression in rheumatoid arthritis patients enrolled in the cooperative systematic disease program randomized clinical trial of methotrexate, auranofin or a combination of the two. Arthritis Rheum 1993;36:1364-9.

27. van der Heijde DM, van Riel PL, Nuver-Zwart IH, Gribnau FW, van de Putte LB: Effects of hydroxychloroquine and sulfasalazine on progression of joint damage in rheumatoid arthritis. Lancet 1989; i:1036-8.

28. Felson DT, Anderson J, Meenan RF. Use of short-term efficacy/toxicity tradeoffs to select second-line drugs in rheumatoid arthritis. A metaanalysis of published trials. Arthritis Rheum 1992; 35:1117-25.

29. Felson DT, Anderson J, Meenan RF. The comparative efficacy and toxicity of second-line drugs in rheumatoid arthritis. Results of two metaanalysis. Arthritis Rheum 1990; 33:1449-61.

30. Haagsma CJ, Russel FGM, Vre TB, van Riel PLCM, van de Putte LBA. Combination of methotrexate and sulphasalazine in patients with rheumatoid arthritis: $\mathrm{Pharmacokinetic}$ analysis and relationship to clinical reponse. Br J Cin Pharmacol 1996; 42:195-200.

31. Moreland LW, Kimberley RP, Alarcon GS. European and US rheumatologists agree in triple but not on double or single early DMARD choice for different types of RA. Arthritis Rheum 1997; 40:S218.

32. Scott DL, Dacre JE, Greenwood A, Treasure L, Huskisson EC Can we develop simple response criteria for slow acting antirheumatic drugs? Ann Rheum Dis 1990; 49:196-8.

33. Maetzel A, Wong A, Strand V, Tugwell P, Wells G, Bombardier C. Meta-analysis of treatment termination rates among rheumatoid arthritis patients receiving disease-modifying anti-rheumatic drugs. Rheumatology 2000; 39:975-81.

34. Smythe CJ. Therapy of rheumatoid arthritis. A pyramidal plan. Postgrade Med 1972; 51:31-9.

35. Wilske KR, Healey LA. Remodelling the pyramid - a concept whose time has come. J Rheumatol $1989 ; 16: 565-7$.

36. Pincus T, Callahan LF: Remodelling the pyramid or remodelling the paradigms concerning rheumatoid arthritis: lessons from Hodgkin's disease and coronary artery disease. J Rheumatol 1990; 17:1582-1585.

37. Pincus T, Wolfe F. Treatment of rheumatoid arthritis: challenges to traditional paradigms. Ann Intern Med 1991; 115:825-7.

38. Kuhn TS. The structure of scientific revolutions, $2^{\text {nd }}$ ed. Chicago: University of Chicago Press, 1970.

39. Bensen WG, Bensen W, Adachi JD, Tugwell PX. Remodelling the pyramid: the therapeutic target of rheumatoid arthritis. J Rheumatol 1990; 17:987-9.

40. Wilke WS, Cogh JD. Therapy for rheumatoid arthritis: combinations of disease-modifying drugs and new paradigms of treatment. Semin Arthritis Rheum 1991; 21:21-34.

41. Emery P, Salmon M. Early rheumatoid arthritis: time to aim for remission? Ann Rheum Dis 1995; 54:944-7.

42. Pincus T, Marcum SB, Callahan LF. Long-term drug therapy for rheumatoid arthritis in seven rheumatology private practices: II Second line drugs and prednisone. J Rheumatol 1992; 19:1885-94.

43. Münster T, Furst DE. Pharmacotherapeutic strategies for disease-modifying antirheumatic drug combinations to treat rheumatoid arthritis. Cin Exp Rheumatol 1999; 17 (suppl 18): 29-36. 
44. Joint committee of the Medical Research Council and Nuffield Foundation. A comparison of prednisolone with aspirin or other analgesics in the treatment of rheumatoid arthritis. Ann Rheum Dis 1959; 18:173-87.

45. Hawley DJ, Wolfe F, Pincus T. Use of combination therapy in the routine care of patients with rheumatoid arthritis: physician and patient surveys. Cin Exp Rheumatol 1999; 17 (suppl 18):78-82.

46. Boers M, Verhoeven AC, Markusse HM, van de Laar MAFJ, Westhovens R, van Denderen JC, van Zeben D, Dijkmans BAC, Peeters AJ, Jacobs P, van den Brink HR, Schouten HJA, van der Heijde DMFM, Boonen A, van der Linden S. Randomised comparison of combined step-down prednisolone, methotrexate and sulphasalazine with sulphasalazine alone in early rheumatoid arthritis. Lancet 1997; 350:309-18.

47. van der Heide A, Jacobs JWG, Bijlsma JWJ, Heurkens AHM, van Booma-Frankfort C, van der Veen MJ, Haanen HCM, Hofman DM, van Albada-Kuipers GA, ter Borg EJ, Brus HL, Dinant HJ, Kruize AA, Schenk Y. The effectiveness of early treatment with "second-line" antirheumatic drugs: a randomized, controlled trial. Ann Intern Med 1996; 124:699-707.

48. Tugwell P. Combination therapy in rheumatoid arthritis: metaanalysis. J Rheumatol 1996; 44 suppl: 43-6.

49. Felson DT, Anderson JJ, Meenan RF: The efficacy and toxicity of combination therapy in rheumatoid arthritis: a meta-analysis. Arthritis Rheum 1994; 37:1487-91.

50. Lukert BP, Raisz LG. Glucocorticoid-induced osteoporosis: Pathogenesis and management. Ann Intern Med 1990: 112:352-64.

51. Olbricht T, Benker G. Glucocorticoid-induced osteoporosis: Pathogenesis prevention and treatment, with special regard to the rheumatic diseases. J Intern Med 1993: 234:237-44.

52. Saag KG, Koehnke R, Caldwell JR, Brasington R, Burmeister LF, Zimmerman B, Kohler JA, Furst DE. Low dose long-term corticosteroid therapy in rheumatoid arthritis: an analysis of serious adverse events. Am J Med 1994; 96:115-23.

53. Morrison $\mathrm{E}$, Capell $\mathrm{H}$. Corticosteroids in the management of rheumatoid arthritis. $\mathrm{Br} \mathrm{J}$ Rheumatol 1996; 35:1-4.

54. Kirwan JR, Arthritis and Rheumatism Low-dose Glucocorticoid Study Group. The effect of glucocorticoids on joint destruction in rheumatoid arthritis. N Engl J Med 1995; 333:142-6.

55. Adinoff $\mathrm{AD}$. Hollister JR. Steroid-induced fractures and bone loss in patients with asthma. NEngl J Med 1983: 309:265-8.

56. Verstraeten A, Dequeker J. Vertebral and peripheral bonemineral content and fracture evidence in postmenopausal patients with rheumatoid artritis; effect of low dose corticosteroids. Ann Rheum Dis 1986; 28:361-8.

57. American College of Rheumatology Task Force on Osteoporosis Guidelines. Recommendation for the prevention and treatment of glucocorticoid-induced osteporosis. Arthritis Rheum 1996; 39:1791-1801.

58. Saag KG, Criswell LA, Sems KM, Nettleman MD, Kolluri S. Low-dose corticosteroids in theumatoid arthritis. Arthritis Rheum 1996; 39: 1818-25.

59. Gough AK, Lilley J, Eyre S, Holder RL, Emery P. Generalised bone loss in patients with early rheumatoid arthritis. Lancet 1994; 344:23-7.

60. Verhoeven AC, Boers M, Tugwell P. Combination therapy in rheumatoid arthritis: updated systematic review. Br J Rheumatol 1998; 37:612-9.

61. Verhoeven AC, Bibo JC, Boers M, Engel GL, van der Linden S. Cost-effectiveness and cost-utility of combination therapy in early rheumatoid arthritis. Randomized comparison of combined step-down prednisolone, methotrexate and sulphasalazine with sulphasalazine alone. Br J Rheumatol 1998; 37:1102-9.

62. Verhoeven AC, Boers M. Limited bone loss due to corticosteroids; a systematic review of prospective studies in rheumatoid arthritis and other diseases. J Rheumatol 1997; 24:1495-503.

63. Verhoeven AC, Boers M, te Koppele JM, van der Laan WH, de Roos J, van der Linden S. Reliability of spot samples for assessment of urinary excretion of pyridinoline in patients with rheumatoid arthritis. Clin Exp Rheumatol 2001; 19:78-80. 
64. Verhoeven AC, Boers M, te Koppele JM, van der Laan WH, Markusse HM, Geusens P, van der Linden S. Bone turnover, joint damage and bone mineral density in early rheumatoid arthritis treated with combination therapy including high-dose prednisolone. Rheumatology 2001; 40:1231-7.

65. Anderson J, Wells G, Verhoeven AC, Felson DT. Factors predicting response to treatment in rheumatoid arthritis: the importance of disease duration. Arthritis Rheum 2000; 43:22-9.

66. Verhoeven AC, Boers M, van der Linden S. Responsiveness of the core-set, response criteria and utility measures in early rheumatoid arthritis. Ann Rheum Dis 2000; 59:966-74.

67. Boers M, Verhoeven AC, van der Linden S. ACR improvement criteria should only be calculated from scores that decrease on improvement. Arthritis Rheum 2001; 44:1052-5.

68. Verhoeven AC, Boers M, van der Linden S. Validity of the MACTAR as functional index in a rheumatoid arthritis trial. J Rheumatol 2000; 27:2801-9.

69. Boers M, Ramsden M. Longacting drug combinations in rheumatoid arthritis: a formal overview. J Rheumatol 1991; 18:316-24.

70. Tugwell $\mathrm{P}$, Boers M. Longacting drug combinations in rheumatoid arthritis: Updated overview. In: Wolfe F, Pincus T, eds. Rheumatoid arthritis: Pathogenesis, assessment, outcome and treatment. New York: Marcel Dekker 1994: 357-71.

71. Smythe HA, Helewa A, Goldsmith CH. Independent assessor' and 'pooled index' as techniques for measuring treatment effects in rheumatoid arthritis. J Rheumatol 1977; 4:144-52.

72. Sharp JT, Lidsky MD, Collins LC, Moreland J. Methods of scoring the progression of radiologic changes in rheumatoid arthritis: Correlation of radiologic, clinical an d laboratory abnormalities. Arthritis Rheum 1971;14:706-20.

73. van der Heijde D. How to read radiographs according to the Sharp/van der Heijde method. J Rheumatol 2000; 27:261-3.

74. Carke AE, Zowall H, Levinton C, Assimakopoulos H, Sibley JT, Haga M, Shiroky J, Neville C, Lubeck DP, Grover SA, Esdaile JM. Direct and indirect medical costs incurred by Canadian patients with rheumatoid arthritis; a 12 year study. J Rheumatol 1997; 24:1051-60.

75. Barrett EM, Scott DGI, Wiles NJ, Symmons DPM. The impact of rheumatoid arthritis on employment status in the early years of disease: a UK community-based study. Rheumatology 2000; 39:1403-1409.

76. Bennett K, Torrance GW, Tugwell P. Methodological challenges in the development of utility measure of health-related quality of life in rheumatoid arthritis. Control Cin Trials 1991; 12 suppl:118-28.

77. Torrance GW. Utility approach to measuring health-related quality of life. J Chronic Dis 1987; 40:593603.

78. Von Neumann J, Morgenstern O. Theory of games and economic behaviour, Princeton: Princeton University Press, 1947, $2^{\text {nd }}$ ed.

79. Laan RF, van Riel PL, van de Putte LB, van Eming LJ, van ' $t$ Hof MA, Lemmens JA. Low dose prednisone induces rapid reversible axial bone loss in patients with rheumatoid arthritis: A randomized, controlled trial. Ann Int Med 1993; 119:963-8.

80. van Schaardenburg D, Valkema R, Dijkmans BAC, Papapoulos S, Zwinderman AH, Han $\mathrm{KH}$, Pauwels EK, Breedveld FC. Prednisone treatment of elderly-onset rheumatoid arthritis; disease activity and bone mass in comparison with chloroquine treatment. Arthritis Rheum 1995; 38:334-42.

81. Gough AKS, Peel NFA, Eastell R, Holder RL, Lilley J, Emery P. Excretion of pyridium crosslinks correlates with disease activity and appendicular bone loss in early rheumatoid arthritis. Ann Rheum Dis 1994; 53:14-7.

82. Tugwell $\mathrm{P}$, Bombardier C. A methodological framework for developing and selecting endpoints in clinical trial. J Rheumatol 1982; 9:758-62.

83. Felson DT. Choosing a core set of disease activity measures for rheumatoid arthritis clinical trials. J Rheumatol. 1993; 20:531-4.

84. Boers M, van Riel PL, Felson DT, Tugwell P. Assessing the activity of rheumatoid arthritis. Baillières Cin Rheumatol 1995; 9:305-17. 
85. Fried BJ, Boers M, Baker PR. A method for achieving consensus on rheumatoid arthritis outcome measures: the OMERACT conference process. J Rheumatol 1993; 20:548-51.

86. Goldsmith CH, Boers M, Bombardier C, Tugwell P, for the Omeract Committee: Criteria for clinically important changes in outcomes: development, scoring, and evaluation of the rheumatoid arthritis patient and trial profiles. J Rheumatol 1993; 20:561-565.

87. Boers M, Tugwell P, Felson DT, van Riel PL, Kirwan JR, Edmonds JP, Smolen JS, Khaltaev N, Muirden KD. World Health Organisation and International League of Associations for Rheumatology core endpoints for symptom modifying antirheumatic drugs in rheumatoid arthritis clinical trials. J Rheumatol 1994; 41 suppl:86-9.

88. Felson DT, Anderson J, Boers M, Bombardier C, Chernoff M, Fried B, Furst D, Goldsmith C, Kieszak S, Lightfoot R, et al. The American College of Rheumatology preliminary core set of disease activity measures for rheumatoid arthritis clinical trials. The Committee on Outcome Measures in Rheumatoid Arthritis Cinical Trials. Arthritis Rheum 1993; 36:729-40.

89. Buchbinder R, Bombardier C, Yeung M, Tugwell P. Which outcome measures should be used in rheumatoid arthritis clinical trials? Cinical and quality-of-life measures' responsiveness to treatment in a randomized controlled trial. Arthritis Rheum. 1995; 38:1568-80.

90. Felson DT, Anderson J, Lange ML, Wells G, LaValley MP. Should improvement in rheumatoid arthritis clinical trials be defined as fifty percent or seventy percent improvement in core set measures, rather than twenty percent? Arthritis Rheum 1998; 41:1564-70.

91. Van der Heide A, Remme CA, Hofman DM, Jacobs JWG, Bijlsma JWJ. Prediction of progression of radiologic damage in newly diagnosed rheumatoid arthritis. Arthritis Rheum 1995; 38:1466-74.

92. Egsmose C, Lund B, Borg G, Pettersson H, Berg E, Brodin U, Trang L. Patients with rheumatoid arthritis benefit from early 2 nd line therapy: 5 year follow up of a prospective double-blind placebo controlled study. J Rheumatol 1995; 22:2208-13.

93. Felson DT, Anderson JJ, Boers M, Bombardier C, Furst D, Goldsmith C, Katz LM, Lightfoot R Jr, Paulus H, Strand V, et al. American College of Rheumatology. Preliminary definition of improvement in rheumatoid arthritis. Arthritis Rheum 1995; 38:727-35.

94. van Gestel AM, Prevoo ML, van ' $t$ Hof MA, van Rijswijk MH, van de Putte LB, van Riel PL. Development and validation of the European League Against Rheumatism response criteria for rheumatoid arthritis. Comparison with the preliminary American College of Rheumatology and the World Health Organisation/ International League Against Rheumatism Criteria. Arthritis Rheum 1996; 39:34-40.

95. Fries JF, Spitz P, Kraines RG, Holman HR. Measurement of patient outcome in arthritis. Arthritis Rheum 1980; 23:137-145.

96. Meenan RF, Gertman PM, Mason JH. Measuring health status in arthritis: The Arthritis Impact Measurement Scales. Arthritis Rheum 1980; 23:146-152.

97. Tugwell P, Bombardier C, Buchanan WW, Goldsmith CH, Grace E, Hanna B. The MACTAR patient preference disability questionnaire: an individualized functional priority approach for assessing improvement in physical disability in clinical trials in rheumatoid arthritis. J Rheumatol 1987; 14:446-51.

98. Boers M, Brooks P, Strand CV, Tugwell P. The OMERACT filter for outcome measures in rheumatology. J Rheumatol 1998; 25:198-9. 


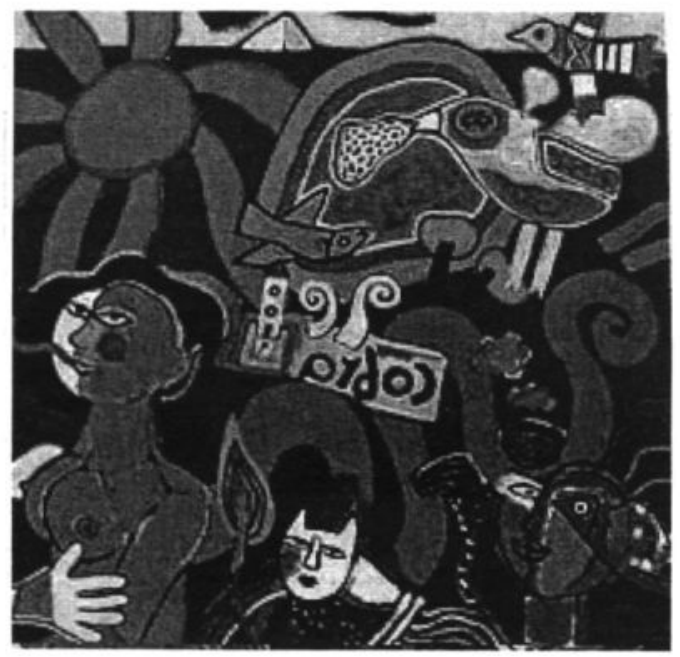




\section{CHAPTER 2}

\section{COMBINATION THERAPY}

IN RHEUMATOID ARTHRITIS:

UPDATED SYSTEMATIC REVIEW

Arco Verhoeven, Maarten Boers, Peter Tugwell

British Journal of Rheumatology 1998; 37: 612-9. 


\title{
Combination Therapy in Rheumatoid Arthritis: Updated Systematic Review
}

\begin{abstract}
In a second update of a systematic review, many new developments in the combined drug treatment of rheumatoid arthritis (RA) are highlighted. In early RA patients, step-down bridge therapy that includes corticosteroids leads to much enhanced efficacy at acceptable or low toxicity. The effects on joint damage may be persistent, but the symptomatic effects are probably dependent on continued corticosteroid dosing. In late patients, cyclosporin improves a suboptimal clinical response to methotrexate, and the triple combination of methotrexate, sulphasalazine, and hydroxychloroquine appears to be clinically better than the components. Other combinations are either untested, tested at low sample size, or show negative interaction. In view of the low volume of evidence, most studies need confirmation by replication.
\end{abstract}




\section{Introduction}

There is a trend among rheumatologists to treat rheumatoid arthritis (RA) patients earlier and more aggressively. New scientific evidence supports early intervention with disease-modifying antirheumatic drug (DMARD) therapy [1]. Rapid and adequate control of disease activity is aimed at prevention of structural joint damage and subsequent loss of function and quality of life. In this setting, combining of so-called DMARDs might lead to additive effects. Alternatively, doses might be reduced, and perhaps some of the toxicity avoided. Many rheumatologists already prescribe combination therapy, although until recently scientific evidence to support this policy was lacking. Over the last years, an increasing number of high-quality trials has been published. We present a second update of a systematic review of combination therapy in RA [2,3].

In combining DMARDs, three main strategies can be distinguished. In this review, the label 'step-up strategy' is reserved for trials in which patients with insufficient clinical benefit from one second-line agent continued the use of this first drug and had another (or placebo) added to this. The label 'parallel' was assigned to trials in which the patients started with a combination of new drugs, and 'step-down' to trials with sequential withdrawal of simultaneously started drugs, prescribed by protocol.

\section{Methods}

\section{Study identification}

The MEDLINE database was searched from August 1992 (the closing date of the previous review) to July 1997 using the MeSH headings: 'arthritis, rheumatoid'; and 'drug therapy, combination'. The bibliographies of all retrieved articles were scrutinized for additional studies. The first authors of studies published only in abstract were contacted. Such studies were eligible for inclusion if a full manuscript was available. Titles and abstracts (when available) were screened by one author (MB up to August 1992, AV subsequently) and any article in English, French, German, or Dutch that appeared potentially relevant was retrieved.

\section{Study selection and validity assessment}

First, the quality of the studies and, thus the strength of evidence was scored on a three-point scale on the basis of two primary criteria: randomisation and blinding. Accordingly, strong evidence came from randomised, double-blind studies; moderately strong evidence from studies that were randomised, but open or partially blinded; and weak evidence from all other studies. This score specified the maximum strength we felt a study could yield. A second set of criteria, modified from Sackett et al [4] was then applied. These were: (a) adequate outcome assessment (blind and comprising toxicity); (b) adequate description of study patients (report of at least age and sex, some record on the previous disease severity and concurrent medication); (c) adequate description of the therapeutic maneuver (i.e. minimal potential of bias, with blinding, contamination, cointervention and compliance properly addressed); (d) complete accounting of study patients in the results. To obtain the final quality score, points were subtracted for each of these criteria not met. 


\section{Data extraction and analysis}

The results of the trials yielding moderately strong or strong evidence in the previous reviews (original and update) were added to the information from the new search. Data extracted from the selected studies included baseline patient characteristics, study and concomitant treatment, outcome measures, and details on toxicity, withdrawals and eligibility criteria for disease activity.

Cinical efficacy, i.e. improvement in clinical outcome measures, was compared between the combined-treatment group and the single-treatment group. In the case of more than one control group, comparisons were made between the combined-treatment group and each control group, but eventually the comparison with the best performing control group was decisive. The WHO/ ILAR core set measures [5] assessed efficacy. These measures comprise tender and swollen joint count (or a score), pain assessment, patient and physician (or observer) global assessment, physical function index [here, in every case Health Assessment Questionnaire (HAQ) or a modification thereof], and acute-phase reaction [i.e. erythrocyte sedimentation rate (ESR) or G-reactive protein (CRP)]. When less than four of these measures had been assessed, first grip strength and second morning stiffness were selected as well. Four levels of efficacy were distinguished based on differences in improvement in the selected measures:

- combined treatment 'substantially more effective' ('++' in the summary table): significantly greater improvement in the combined-treatment group in at least half of the selected measures (minimum two out of four), plus improvement at least $150 \%$ that of the control group;

- combined-treatment 'more effective' ('+): significantly greater improvement in at least half of the selected measures;

- 'positive trend' ('+?): significantly greater improvement in at least $25 \%$ of the measures, or significantly greater improvement only in a predefined summary index of measures;

- 'no difference' ('=): the remainder.

When the total number of core set measures (plus grip strength and morning stiffness) was less than four, only trends were scored. Toxicity was rated as increased ('+) when significantly more patients from the combined treatment group were withdrawn from the study medication because of adverse events. Likewise, it was rated as decreased ('-) when significantly less patients from the combined treatment group were withdrawn from the study medication because of adverse events. A significant difference (or trend) in numbers of adverse events not leading to withdrawal was rated as 'trend of more toxicity' ('+?') or 'trend of less toxicity' ('-?). Where possible, results of statistical tests comparing the effect or toxicity of the different treatments were calculated or recalculated using the reported data. 


\section{Results}

Previous work had yielded eight relevant studies [2,3]. Six of these eight provided 'strong' or 'moderate strong evidence' and are included in the final selection $[6,12,14,19,20,24]$. The current search, covering the interval between August 92 and July 97, yielded 231 new citations. Together with previous reviews, this brings the total to 611 titles scanned, 100 were retrieved and 18 selected for review. Of the screened abstracts and titles, 38 were linked to a possibly relevant article. Three articles in Japanese [26-28] were not rated. Not selected articles were case reports, editorials, observational or non-randomised studies. Three studies that described adjuvant treatment with oral corticosteroids, androgens and oestrogens, respectively, were not included because they failed to meet the criterion of a one-type single DMARD control group [29-31]. Two studies described an extended follow-up or radiological assessments of an already selected article [32,33]; the data from these publications were added to those of the original study $[19,20]$. Two articles were found in the reference list of selected articles $[9,10]$. Four possibly relevant reports in abstract form were found in abstract book supplements. The corresponding manuscripts of two recently published articles were obtained $[21,22]$.

The total of 20 (six old, 14 new) included trials are listed in Table 2.1. The total number of patients included in these trials is 1952 . All trials used a more or less strict criterion to verify the presence of active disease. The studies are ranked according to treatment strategy as well as the DMARDs of choice. Six studies describe a step-up strategy; two of these used cyclosporin, three used i.m. gold as anchor drug, and two methotrexate. Ten studies describe a parallel strategy; of these, six used methotrexate (all but one as anchor drug), six studies used antimalarials (one as anchor drug), three used sulphasalazine, one i.m. gold, dapsone or D-penicillamine (as anchor drugs); also used were auranofin and azathioprine (as additional drug; total more than 10, due to combinations). The studies with a step-down strategy (four in total) all used steroids (i.m. methylprednisone pulses or predniso(lo)ne orally). Steroids were added to i.m. gold (in two studies) or sulphasalazine (also in two studies; in one study prednisolone was added together with methotrexate).

\section{Studies with step-up strategy}

Smyth et al [6] added $75 \mathrm{mg} / \mathrm{d}$ cyclophosphamide or placebo to a stable and continued pretrial dose of prednisone varying between 3 and $15 \mathrm{mg} /$ day in 29 patients with established disease. After 6 months, outcomes in the combined-treatment group were significantly more improved in grip strength and an inflammatory index comprising swelling, redness, pain on motion, heat and tenderness, but not in ESR. Only one case of alopecia was reported in the combined-treatment group with no withdrawal due to toxicity in either group. Given the paucity of outcomes, this suggests a trend of increased efficacy with no increase of toxicity, but the disease activity at baseline was less in the placebo group. 
Table 2.1: Reviewed trials clustered by drug combination and combination strategy.

first publ. $n \quad n \quad \begin{gathered}\text { compared } \\ \text { drugs or }\end{gathered}$ therapy $\begin{gathered}\text { strength disease assess } \\ \text { of }\end{gathered}$ duration ment ' $\mathrm{O}$ ' effi toxi author ref. year pts groups combinations strategy evidence (yrs) (mo) score cacy city

\begin{tabular}{|c|c|c|c|c|c|c|c|c|c|c|c|c|}
\hline Smyth & [6] & 75 & 29 & 2 & $\begin{array}{l}\text { (Pred CyP) } \\
\text { vs Pred }\end{array}$ & step-up & moderate & $>2$ & 6 & $2 / 3$ & + & $=$ \\
\hline Moreland & [7] & ‘95 & 64 & 4 & $\begin{array}{c}\text { (MTX aCD4) } \\
\text { vs MTX }\end{array}$ & step-up & strong & 9 & 3 & $0 / 6$ & $=$ & $=$ \\
\hline Tugwell & [8] & '95 & 148 & 2 & $\begin{array}{l}\text { (MTX CyA) } \\
\text { vs MTX }\end{array}$ & step-up & strong & 10 & 6 & $6 / 7$ & ++ & $=$ \\
\hline Bendix & [9] & ‘96 & 40 & 2 & $\begin{array}{c}(\mathrm{AU} \mathrm{CyA}) \\
\text { vs } \mathrm{AU}\end{array}$ & step-up & strong & 11 & 6 & $1 / 7 *$ & $=$ & $+?$ \\
\hline Yasuda & [10] & ‘94 & 24 & 2 & $\begin{array}{l}\text { (AU Buc) } \\
\text { vs } \mathrm{AU}\end{array}$ & step-up & strong & 8 & 3 & $3 / 4$ & + & $+?$ \\
\hline Porter & [11] & '93 & 142 & 2 & $\begin{array}{c}\text { (AU Hcq) } \\
\text { vs } \mathrm{AU}\end{array}$ & step-up & moderate & 6 & 6 & $0 / 4$ & $=$ & $=$ \\
\hline Scott & [12] & '88 & 101 & 2 & $\begin{array}{c}\text { (AU Hcq) } \\
\text { vs } \mathrm{AU}\end{array}$ & parallel & strong & 2 & 12 & $1 / 4^{\dagger}$ & $+?$ & $+?$ \\
\hline Faarvang & [13] & 93 & 91 & 3 & $\begin{array}{c}(\mathrm{SSZ} \mathrm{Hcq}) \\
v s \mathrm{SSZ} \text { vs Hcq }\end{array}$ & parallel & strong & 7 & 6 & $0 ; 2 / 5$ & $=$ & $=$ \\
\hline Gibson & [14] & '87 & 72 & 3 & $\begin{array}{c}\text { (Dpen Cq) } \\
v s \text { Dpen } v s \mathrm{Cq}\end{array}$ & parallel & moderate & 2 & 12 & $0 ; 1 / 5$ & $=$ & $=$ \\
\hline Haar & [15] & '93 & 80 & 3 & $\begin{array}{l}\text { (Daps Hcq) } \\
\text { vs Daps vs Hcq }\end{array}$ & parallel & strong & 2 & 6 & $1 ; 1 / 4$ & $=$ & $=$ \\
\hline Trnavsky & [16] & '93 & 40 & 2 & $\begin{array}{c}\text { (Heq MTX) } \\
\text { vs Hcq }\end{array}$ & parallel & strong & $>2$ & 6 & $2 / 5$ & $+?$ & $+?$ \\
\hline Ferraz & [17] & '94 & 82 & 2 & $\begin{array}{l}\text { (MTX Cq) } \\
\text { vs MTX }\end{array}$ & parallel & strong & 8 & 6 & $2 / 4$ & + & $+?$ \\
\hline O’Dell & [18] & '96 & 102 & 3 & $\begin{array}{l}\left(\mathrm{MTX} \mathrm{SSZ}^{\ddagger} \mathrm{Hcq}\right) \\
\left(\mathrm{SSZ}^{\ddagger} \mathrm{Hcq}\right) \text { vs MTX }\end{array}$ & $\mathrm{X}^{\text {parallel }}$ & strong & 9 & 9 & $2 ; 2 / 5$ & $+?$ & $=$ \\
\hline Williams & [19] & '92 & 335 & 3 & $\begin{array}{c}\text { (MTX AUR) } \\
\text { vs MTX vs AUR }\end{array}$ & parallel & strong & 5 & 12 & $0 ; 0 / 5$ & $=$ & $+?$ \\
\hline Willkens & [20] & ${ }^{\prime} 92$ & 209 & 3 & $\begin{array}{c}\text { (MTX AZA) } \\
v s \text { MTX vs AZA }\end{array}$ & parallel & strong & 8 & 12 & $0 ; 2 / 6$ & $=$ & $+?$ \\
\hline Haagsma & [21] & '97 & 105 & 3 & $\begin{array}{c}\text { (MTX SSZ) } \\
v s \mathrm{MTX} v s \mathrm{SSZ}\end{array}$ & parallel & strong & $<1$ & 6 & $0 ; 0 / 7$ & $=$ & $=$ \\
\hline Boers & [22] & '97 & 155 & 2 & $\begin{array}{c}\text { (SSZ MTX Predl) } \\
\text { vs SSZ }\end{array}$ & step-down & strong & $<1$ & 6 & $6 / 7$ & ++ & - \\
\hline van Gestel & [23] & '95 & 40 & 2 & $\begin{array}{c}\text { (AU Pred) } \\
\text { vs } \mathrm{AU}\end{array}$ & step-down & strong & 2 & 3 & $3 / 6$ & ++ & $=$ \\
\hline Corkill & [24] & ${ }^{\circ} 90$ & 59 & 2 & $\begin{array}{c}\text { (AU MP) } \\
v s \mathrm{AU}\end{array}$ & step-down & strong & 6 & 3 & $3 / 4$ & ++ & $+?$ \\
\hline Ciconelli & [25] & '96 & 38 & 2 & $\begin{array}{c}\left(\mathrm{SSZ} \mathrm{MP}^{5}\right) \\
\text { vs } \mathrm{SSZ}\end{array}$ & step-down & moderate & 6 & 6 & $0 / 4$ & $=$ & $=$ \\
\hline
\end{tabular}


' $O$ ' score is the number of clinical WHO/ILAR core set outcome measures (see Methods) significantly better in the combined-treatment group in comparison with control group(s). Results from comparisons with two control groups are separated by a semicolon (;). Behind the slash (/) is the total number of assessed core set measures. NB. The efficacy rating is derived from the $\mathrm{O}$ score, and in some cases also from improvement in 2 non-core set measures (grip strength and morning stiffness) or from improvement in a predefined primary outcome index.

AU, i.m. gold salts; AUR, auranofin; AZA, azathioprine; Buc, bucillamine; aCD4, monoclonal anti-CD4 antibodies (three doses); $\mathrm{Cq}$, chloroquine; CyA, cyclosporin; CyP, cyclophosphamide; Daps, dapsone; Dpen, D-penicillamine; Hcq, hydroxychloroquine; MP, methylprednisone pulses intravenously; MTX, methotrexate; Pred, prednisone; Predl, prednisolone; SSZ, sulphasalazine.

*; Non-core set measures patient's and (non-blind) clinician's assessment of overall efficacy did show significantly more improvement in the combined-treatment group.

$\dagger$; The combined-treatment group showed significantly better scores for a validated disease activity index based on five variables in a flow chart.

\$. Low sulphasalazine doses in the combined-treatment groups; $1 \mathrm{~g} / \mathrm{d}$.

§; Low methylprednisone doses: $5 \mathrm{mg} / \mathrm{Kg} /$ pulse.

Moreland et al [7] performed a dose-finding study of monoclonal anti-CD4 antibody cMT412 in three different doses or placebo added to stable treatment with methotrexate ( $\leq 15$ $\mathrm{mg} /$ week) in 64 patients with refractory RA. Assessments after 3 months treatment and 4 i.v. pulses anti-CD4 did not show any relevant between-group difference in clinical efficacy or toxicity.

Tugwell et al [8] added cyclosporin or placebo to methotrexate in 148 patients with established disease and insufficient response to methotrexate alone. After 6 months, all outcomes with exception of ESR were substantially and significantly better in the combined-treatment group (HAQ and global assessments $P<0.001$ ). Expressed in percentages, improvement as compared with placebo varied between 19 and $26 \%$. The frequency of adverse effects was similar to prior trials of methotrexate and cyclosporin used alone. A threshold of $30 \%$ increase in serum creatinine for dose reduction resulted in a relatively low mean cyclosporin dose $(3 \mathrm{mg} / \mathrm{kg})$. Eighty per cent of the included patients had stable co-medication with low-dose corticosteroids ( $\leq 10 \mathrm{mg})$.

Bendix and Bjelle [10] added cyclosporin or placebo to i.m. gold treatment in 40 patients. After 6 months, the combined treatment showed increased efficacy only in patient's global assessments of overall health and clinical efficacy, and non-blind assessments by a treating physician $(P<0.01$ and $<0.05)$; other core set measures, including blinded observer's global assessment, showed no difference. No serious adverse effects were noted. Higher blood pressure and signs of renal function impairment were found more often in the cyclosporin-treated group, also dose reduction was required significantly more often in the cyclosporin group. Adverse events requiring symptomatic treatment occurred only in eight patients; four in each treatment group. Six months after the end of combination therapy, all differences had disappeared. 
Yasuda et al [10] added bucillamine, a drug developed in Japan, or placebo to i.m. gold treatment in 24 patients. After 3 months, the combined-treatment group had significantly better outcomes in swollen joint count, physician's global assessment and CRP $(P<0.05)$, and similar outcomes (trend) in tender joint count and ESR. Withdrawal for lack of efficacy only occurred in the control group (five patients), and withdrawal due to toxicity occurred more often in the combined-treatment group ( 5 vs 3).

Porter et al [11] added hydroxychloroquine or placebo to i.m. gold treatment in 142 patients. After 6 months, no differences were evident between the groups. Withdrawal (for all reasons) was comparable in both trial groups (approximately 28\%). Owing to the lack of description of previous medication and patient compliance, the strength of evidence was rated as moderate.

\section{Studies with parallel strategy}

Scott et al [12] also tested the combination hydroxychloroquine and i.m. gold, but in a parallel strategy against i.m. gold alone in 101 patients. After 12 months of treatment the combination showed a positive trend in all of the outcomes, but only CRP $(P=0.01)$ and the a priori defined composed summary index of disease activity were significantly better $(P<0.05)$. There was less progression of joint damage on radiographs in the combined-treatment group, although this did not reach significance. The total withdrawal rate was high: $42 \%$. The authors report that toxicity might be enhanced as 18 patients in the combined-treatment group vs 10 in the control group were withdrawn for adverse effects.

Faarvang et al [13] compared the combination of sulphasalazine and hydroxychloroquine with each of these agents alone in 91 patients. Analysis of study completers after 6 months treatment showed no difference between combined treatment and single sulphasalazine treatment. However, combined treatment did show better outcomes in swollen joint count and patient's global compared to hydroxychloroquine alone $(P<0.05)$. In our view, this only confirms that sulphasalazine is a more effective drug than hydroxychloroquine. Both groups showed similar progression of joint damage on radiographs. Withdrawal, for adverse effects as well as other reasons, in this trial was frequent in all treatment groups (32\%).

Gibson et al [14] compared the combination of D-penicillamine and chloroquine in comparison with each of these alone in 72 patients. After 12 months, the decreases in ESR in the combined-treatment group were significantly larger compared to chloroquine, but not compared to D-penicillamine. Improvements in morning stiffness, joint tenderness and swollen joint score and grip strength did not show significant contrasts between treatment groups. There were significantly more adverse effects in the combined-treatment and the D-penicillamine groups compared with chloroquine.

Haar et al [15] compared the combination of hydroxychloroquine and dapsone with each of these drugs alone in 80 patients. After 6 months of treatment, the combination showed a positive trend with a significant difference only in one measure: ESR, but the baseline values for ESR were also better in this treatment group. Patients treated with combined dapsone and hydroxychloroquine showed less progression of joint damage, but this result was weakened by serious disbalance between groups in baseline values. Withdrawals for toxicity were more numerous in the combined-treatment group but this difference was not significant ( 8 vs 3 and $4 ; P=0.11$ ). 
Trmavsky et al [16] compared the combination of hydroxychloroquine and methotrexate with hydroxychloroquine and placebo in 40 patients. After 6 months, the combined treatment showed a positive trend with significantly better outcomes in two of five core set measures; patient's global and ESR $(P<0.05)$. The combined-treatment group contained more patients without progression of joint damage, but the report allows no conclusion on wether this difference was significant. Withdrawal for adverse effects was rare (one case).

Ferraz et al [17] compared the combination of methotrexate and chloroquine to methotrexate alone in 82 patients. After 6 months, the combination was more effective in tender joint count $(P$ $=0.04)$ and HAQ $(P=0.04)$. The authors state that combined treatment was slightly more toxic (and effective), although only three patients were withdrawn due to adverse effects (two of whom had combined treatment). The percentage loss to follow-up (partially related to non-compliance) was quite high $(9 \%)$ in this study.

O'Dell et al [18] compared the combination of methotrexate, sulphasalazine and hydroxychloroquine to the combination of sulphasalazine and hydroxychloroquine, and to methotrexate alone, in 102 patients. The dose of sulphasalazine ( $1 \mathrm{~g} /$ day) was low. Every 3 months, dose adjustments of methotrexate were allowed, guided by assessments of the effect of therapy. The main assessment of efficacy was after 9 months when no further opportunity was offered to adjust the methotrexate dose in case of insufficient therapy response (by definition: $<50 \%$ improvement in modified Paulus criteria). At this time, 27/31, 23/35 and 28/36 patients were considered responders $\left(\chi^{2}\right.$ test: triple therapy $\imath$ sulphasalazine-hydroxychloroquine $P=0.04 ; \tau$ methotrexate $P=0.32$; overall $P=0.12$ ). Based on survival analyses, the authors report significantly more patients with a response to triple therapy after 9 months and conclude that triple therapy results in enhanced efficacy with no increase of toxicity. Non-responders were considered therapy failures and further report on follow-up was restricted to responders. At 9 months of follow-up and according to the rules of this review, triple therapy only showed a positive trend: significantly better swollen and tender joint counts compared to methotrexate, and significantly better swollen joint count and ESR compared to hydroxychloroquine-sulphasalazine. At $2 \mathrm{yr}$ follow-up (with $38 \%$ patients still in follow-up), the between-treatment group contrasts were larger (and highly significant), but this concerns only the patients who had a sufficient response according to the modified Paulus criteria at month 9. Withdrawal for toxicity was rare at year 2, but data on withdrawals due to adverse events at month 9 were not available; however, overall withdrawal at month 9 was about equal.

Williams et al [19] compared the combination of methotrexate and oral gold $(n=106)$ against treatment with each of these agents alone in 335 patients. After 48 weeks, none of the five assessed core set measures showed more benefit in the combined-treatment group. Withdrawals for toxicity occurred somewhat more frequently in the combined-treatment group ( $21 \%$ vs $15 \%$ and 14\%; trend, not significant). Subsequently, Lopez-Mendez et al [32] reported no differences between the groups in progression of radiographic scores at week 48 . 
Willkens et al [20] compared the combination of methotrexate and azathioprine with each of these drugs alone in 209 patients. Data data on ESR and HAQ were subsequently added in a letter [33], and data on 48 weeks of follow-up and radiologic progression were published later [34]. The combination was not better in the between-group comparisons (withdrawals considered as treatment failures), except for ESR when combined treatment was compared to single azathioprine $(P=0.03)$. The authors also report a trend of less radiographic progression in the methotrexate group. Adverse effects occurred primarily in the combined-treatment and azathioprine group (trend). Numbers on withdrawn patients per treatment group due to toxicity are not available, but therapeutic interventions related to adverse events were reported more often in the combined treatment group ( $48 \%$ vs $25 \%$ and $21 \%$ ).

Haagsma et al [21] compared the combination of methotrexate and sulphasalazine with each of these drugs alone in 105 patients. After 52 weeks, the combination was not more effective in any of the four core set or other measures. Response to treatment was exceptionally good in all groups: $74 \%$ met the preliminary ACR criteria for improvement [35]. Fewer patients were withdrawn for toxicity reasons in the single methotrexate treatment group compared to the combined-treatment group $(P=0.025)$. In contrast with most other trials, the patients included in this study had early disease. These results agree with those of a trial only published in abstract form [36], but contrast with the results of an open trial (not included in this review) in which patients with insufficient reaction to sulphasalazine first stopped this drug and afterwards were randomised to combined methotrexate-sulphasalazine, or methotrexate alone. Here, the combined-treatment group showed significantly better outcomes [37].

\section{Studies with step-down strategy}

Boers et al [22] compared the combination of sulphasalazine $(2 \mathrm{~g} /$ day), methotrexate $(7.5$ $\mathrm{mg} /$ week), and prednisolone (initially $60 \mathrm{mg} /$ day, tapered in 6 weekly steps to $7.5 \mathrm{mg} /$ day) with sulphasalazine alone in 155 patients. The last assessment of therapy effect of combined treatment was at week 28 of follow-up, as prednisolone and methotrexate were tapered and stopped after 28 and 40 weeks, respectively. At week 28 , significant better outcomes in the combined-treatment group were seen in all composite measures and all but one of the core set measures $(P<0.002)$. In these measures, the improvement in the combined-treatment group was twofold or almost twofold that of the single sulphasalazine group. Seventy-two per cent of the patients in the combined-treatment group is $49 \%$ in the sulphasalazine group had improved according to the ACR criteria $(P=0.006)$. The clinical difference between the groups decreased and was no longer significant after prednisolone was stopped, and there were no further changes after methotrexate was stopped. Withdrawal for toxicity during the first 28 weeks was significantly less in the combined-treatment group ( 1 vs 7; $P=0.04$ ). The frequency of adverse events not resulting in withdrawal was similar in both groups. In addition to the clinical results, progression of joint damage in the combined-treatment group was one-third that in the sulphasalazine group. This effect persisted until week 80 , i.e. $1 \mathrm{yr}$ after the withdrawal of prednisolone which started at week 28 of follow-up. 
Van Gestel et al [23] compared the combination of i.m. gold (50 mg/week) and prednisone (initially $10 \mathrm{mg} /$ day for 12 weeks, then tapered to zero in 2-weekly steps) with i.m. gold alone in 40 patients. The main assessment was at week 12 , just before prednisone was gradually withdrawn. At this time, all five assessed core set measures showed significantly greater improvement in the combined-treatment group; the magnitude of this improvement is not reported. The improvement in a composite index , the disease activity score (DAS) [38], in the combined-treatment group was more then twofold that with single gold treatment. Progression of joint damage was similar in both groups. Withdrawal due to toxicity was the same in both groups after 20 weeks (four patients in each group). The authors report troublesome rebound effects in the combined-treatment group after withdrawal of prednisone. This appears to be based on a single significant between-group comparison in an array of 13: in week 20 of follow-up, the DAS in the combined-treatment group was worse than in the control group. However, at this moment (and up to week 44), patients in both groups were still better than at baseline. After 44 weeks ( 32 weeks after the beginning of tapering prednisone), no between-group difference remained.

Corkill et al [24] compared the addition of three pulses of $120 \mathrm{mg}$ i.m. methylprednisone (at week 0,4 and 8) to i.m. gold in 59 patients. After 12 weeks, the combined treatment was significantly better in three of four core set measures; improvement in pain and physical function was more than twice as high, and joint count almost twice as high. After 24 weeks, the betweengroup difference had almost disappeared. Progression of joint damage was similar in both groups. Withdrawal due to toxicity during 24 weeks was more frequent in the combined-treatment group, but not significantly so.

Finally, Ciconelli et al [25] compared the addition of three low-dose i.v. methylprednisone pulses $(5 \mathrm{mg} / \mathrm{kg})$, at baseline, month 1 and 2, with sulphasalazine alone in 38 patients. Eighty per cent of the patients in both groups had a prescription of oral corticosteroids. In the 6 month study period, no differences between treatment groups in efficacy or toxicity were found. The relatively low dose of methylprednisone in a population already treated with corticosteroids may have decreased the possible contrast. This important co-intervention with oral corticosteroids was the reason to rate the strength of evidence from this trial as moderate.

Figure 2.1 summarizes heterogeneity of the findings of this systematical review graphically. Except for corticosteroids, there appears to be no trend for an overall beneficial effect of a particular drug in a combination. The figure also shows the lack of data: low sample size in most trials, and many untested combinations. 


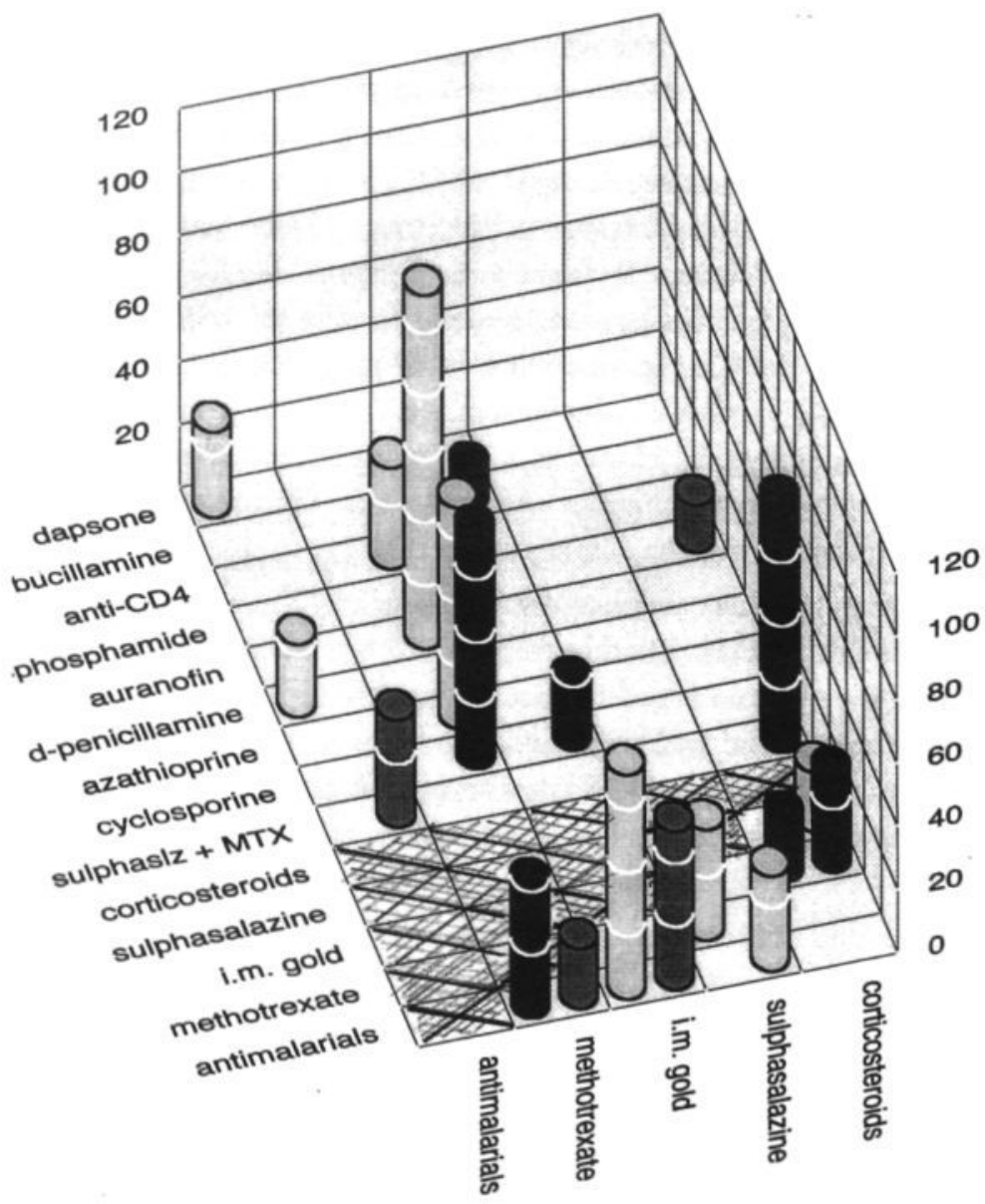

Figure 2.1: Three-dimensional summary of efficacy of combination therapy.

From the perspective of the five most used drugs, the matrix describes the combinations of all single drugs reviewed, and one two-drug combination. Each bar describes a specific combination trial; its length reflects the sample size of the combined-treatment group, its shade reflects the evidence that combined treatment is better than the single drug(s). Dark grey, strong evidence that combination is better or much better, light grey, moderate evidence that combination is better (any evidence or trend); white, no evidence that combination is better. Because the five primary drugs are repeated on the long axis, a dark area in the matrix indicates overlap. 


\section{Discussion}

In its second update since 1991, this review highlights exciting new developments in the combined drug treatment of RA. In early disease, step-down bridge therapy with corticosteroids appears safe and, in the right dose and duration, truly disease modifying; however, the effect on disease activity (not damage) appears dependent on continuation of low-dose corticosteroids. This strengthens Kirwan et al's [29] finding of the damage control resulting from corticosteroid adjuvant therapy, a study not included in this review because anti-rheumatic therapy was not uniformly applied in the control group. In late disease, patients with a suboptimal response to methotrexate clinically improve with cyclosporin, and some patients on triple therapy with methotrexate, sulphasalazine and hydroxychloroquine appear clinically better off than patients on a two-drug combination or methotrexate alone. This lifts the gloom from the other studies compiled so far, where negative interaction (i.e. results of the combination are the same or only slightly better than the single drugs) prevails, often at the cost of somewhat increased toxicity. Interestingly enough, rheumatologists have not waited for these first positive results or heeded the many negative trials; according to two recent surveys published in abstract $[39,40]$ they almost universally embrace combination therapy.

Felson et al [41] recently published a meta-analysis on combination therapy in which he pooled the available data. He found a negative answer to the question: 'Does combination therapy on average make a difference compared to average single therapy?' In our view, the heterogeneity in combinations, strategies and patient material makes this a less interesting research question. As shown in Figure 2.1, each combination needs careful study of its potential in several trials, which can subsequently be pooled.

Despite the results of 'Tugwell et al's study, we feel a step-down or parallel strategy in general shows more potential than a step-up strategy. The reason is that step-up trials select patients that have demonstrated less responsiveness to therapy, thus a priori decreasing the chance of future response. Also, if non-compliance is the basis for lack of efficacy, non-compliant patients are more prone to being selected in a trial with non-responders, and the subsequent therapy will again be more prone to fail.

Although methodology has improved significantly in recent years, we still found a number of problems. Most selected studies had small patient numbers, and notably in the studies with negative results, post hoc sample size calculations were often lacking. In theory, type II errors can be minimized by sufficiently large sample sizes. In practice, it is often hard to find eligible patients. Reliable and responsive measurements can also help to record an actual contrast between groups. For example, with joint score assessments, reliability can be improved by frequent training of the assessors. 
Co-intervention and contamination are important issues in clinical trials and, obviously, any type of co-intervention should be reported in a transparent way. Co-intervention with low-dose corticosteroids was very common in many of the trials selected for this review. Steroids quickly reduce disease activity. With less room for improvement induced by the investigated treatment, demonstration of contrast between treatment groups becomes harder. Corticosteroids are generally known as symptom-relieving drugs. The data summarized here, make it clear that systemic corticosteroids should be considered as disease-controlling anti-rheumatic therapy (DCART) [42]. Accordingly, uncontrolled co-intervention with corticosteroids in RA clinical trials needs reconsideration.

In conclusion, in early RA patients, step-down bridge therapy that includes corticosteroids leads to much enhanced efficacy at acceptable or low toxicity. The effects on joint damage may be persistent, but the symptomatic effects are probably dependent on continued corticosteroid dosing. In late patients, cyclosporin improves a suboptimal clinical response to methotrexate, and the triple combination of methotrexate, sulphasalazine, and hydroxychloroquine appears clinically better than the components. Other combinations are either untested, tested at low sample size, or show negative interaction. In view of the low volume of evidence, most studies need confirmation by replication. 


\section{References}

1. van der Heide A, Jacobs JWG, Bijlsma JWJ, et al. The effectiveness of early treatment with 'secondline' antirheumatic drugs; a randomized, controlled trial. Ann Intern Med 1996; 124:699-707.

2. Boers M, Ramsden M. Long-acting drug combinations in rheumatoid arthritis; a formal overview. J Rheumatol 1991; 18:316-24.

3. Tugwell $P$, Boers M. Long-acting drug combinations in rheumatoid arthritis. Updated overview. In: Wolfe F, Pincus T eds. Rheumatoid arthritis: pathogenesis, assessment, outcome and treatment. New York: Marcel Dekker 1994: 357-71.

4. Sackett DL, Haynes RB, Tugwell P: Deciding on the best therapy. In: Cinical Epidemiology. A basis science for clinical medicine. Boston: Little Brown 1985: 176.

5. Boers M, Tugwell P, Felson DT, et al. World Health Organisation and International League of Associations for Rheumatology core endpoints for symptom modifying antirheumatic drugs in rheumatoid arthritis clinical trials. J Rheumatol 1994; 41 suppl:86-9.

6. Smyth CJ, Bartholomew BA, Mills DM, Steigerwald JC, Strong SJ, Recart S. Cyclophosphamide therapy for rheumatoid arthritis. Arch Intern Med 1975; 135:789-93.

7. Moreland LW, Pratt PW, Mayes MD, et al. Double-blind placebo-controlled multicenter trial using chimeric monoclonal, anti-CD4 antibody, cM-T412, in rheumatoid arthritis patients receiving concomitant methotrexate. Arthritis Rheum 1995; 38:1581-8.

8. Tugwell P, Pincus T, Yocum D, et al. Combination with cyclosporin and methotrexate in severe rheumatoid arthritis. NEngl J Med 1995; 333:137-41.

9. Bendix G, Bjelle A. Adding low-dose cyclosporin A to parental gold therapy in rheumatoid arthritis: a double blind placebo-controlled study. Br J Rheumatol 1996; 35:1142-9.

10. Yasuda M, Sakai K, Oribe M, et al. Efficacy of additive DMARD therapy in patients with rheumatoid arthritis. Double-blind controlled trial using bucillamine and placebo with maintenance doses of gold sodium thiomalate. J Rheumatol 1994; 21:44-50.

11. Porter DR, Capell HA, Hunter J. Combination therapy in rheumatoid arthritis - no benefit of addition of hydroxychloroquine to patients with a suboptimal response to intramuscular gold therapy. J Rheumatol 1993; 20:645-9.

12. Scott DL, Pawes PT, Tunn E, et al. Combination therapy with gold and hydroxychloroquine in rheumatoid arthritis; a prospective, randomized placebo-controlled study. Br J Rheumatol 1989; 28:128-33.

13. Faarvang KL, Egsmose C, Kryger P, Pødenfandt J, Ingeman-Nielsen M, Hansen TM. Hydroxychloroquine and sulphasalazine alone and in combination in rheumatoid arthritis: a randomised double blind trial. Ann Rheum Dis 1993; 32:711-4.

14. Gibson T, Emery P, Amstrong RD, Crisp AJ, Panayi GS. Combined D-penicillamine and chloroquine treatment of rheumatoid arthritis - a comparative study. Br J Rheumatol 1987; 27:279-84.

15. Haar D, Sølvkjær M, Unger B, Rasmussen KJ, Christensen L, Hansen TM A double-blind comparative study of hydroxychloroquine and dapsone, alone and in combination, in rheumatoid arthritis. Scand J Rheumatol 1993; 22:113-8.

16. Trnavsky K, Gatterová J, Lindusková M, Pelísková Z. Combination therapy with hydroxychloroquine and methotrexate in rheumatoid arthritis. Z Rheumatol 1993; 52:292-6.

17. Ferraz MB, Pinheiro GR, Helfenstein M, et al. Combination therapy with methotrexate and chloroquine in rheumatoid arthritis. A multicenter randomized placebo-controlled trial. Scand J Rheumatol 1994; 23:231-6.

18. O'Dell JR, Haire CE, Erikson N, et al. Treatment of rheumatoid arthritis with methotrexate alone, sulfasalazine and hydroxychloroquine, or a combination of all three medications. N Engl J Med 1996; 334:1287-91. 
19. Williams HJ, Ward JR, Reading JC, et al. Comparison of auranofin, methotrexate, and the combination of both in the treatment of rheumatoid arthritis, a controlled clinical trial. Arthritis Rheum 1992; 35:259-69.

20. Willkens RF, Urowitz MB, Stablein DM, et al. Comparison of azathioprine, methotrexate, and the combination of both in the treatment of rheumatoid arthritis. A controlled clinical trial. Arthritis Rheum 1992; 35:1799-806.

21. Haagsma CJ, van Riel PLCM, de Jong AJL, van de Putte LBA. Combination of sulphasalazine and methotrexate versus the single components in early rheumatoid arthritis. $\mathrm{Br}$ J Rheumatol 1997; 36:1082-8.

22. Boers M, Verhoeven AC, Markusse HM, et al. Randomised comparison of combined step-down prednisolone, methotrexate and sulphasalazine with sulphasalazine alone in early rheumatoid arthritis. Lancet 1997; 350:309-18.

23. van Gestel AM, Laan RFJM, Haagsma CJ, van de Putte LBA, van Riel PLCM. Oral steroids as bridge therapy in RA patients starting with parental gold. A randomized double-blind placebo-controlled trial. Br J Rheumatol 1995; 34:347-51.

24. Corkill M, Kirkham BW, Chikanza IC, Gibson T, Panayi GS. Intramuscular depot methylprednisolone induction of chrysotherapy in rheumatoid arthritis: A randomised clinical trial. Br J Rheumatol 1990; 29:274-9.

25. Giconelli RM, Ferraz MB, Visioni RA, Oliveira LM, Atra E. A randomized double-blind controlled trial of sulphasalazine combined with pulses of methylprednisolone or placebo in the treatment of rheumatoid arthritis. Br J Rheumatol 1996; 35:150-4.

26. Fujii T, Suwa A, Yosida T, Mimori T, Akizuki M. Study on combined of auranofin, salazosulfapyridine and methotrexate in rheumatoid arthritis. Ryumachi. 1994; 34:571-82.

27. Minami M, Kaneda K. A long-term clinical analysis of the rheumatoid patients treated by a combination of gold salt therapy and OCA. Ryumachi 1995; 35:780-91.

28. Kashiwazaki S, Akizuki M, Ichikawa Y, et al. Prospective clinical study of the combination therapy of auranofin and methotrexate for rheumatoid arthritis; a multi-center study. Ryumachi 1996; 36:528-44.

29. Kirwan JR, Arthritis and Rheumatism Low-dose Glucocorticoid Study Group. The effect of glucocorticoids on joint destruction in rheumatoid arthritis. N Engl J Med 1995; 333:142-6.

30. Booij A, Biewenga-Booij CM, Huber-Bruning O, Cornelis C, Jacobs JW, Bijlsma JW. Androgens as adjuvant treatment in postmenopausal female patients with rheumatoid arthritis. Ann Rheum Dis 1996; 55:811-5.

31. van den Brink $H R$, van Everdingen AA, van Wijk MJ, Jacobs JW, Bijlsma JW. Adjuvant oestrogen therapy does not improve disease activity in postmenopausal patients with rheumatoid arthritis. Ann Rheum Dis 1993; 52:862-5.

32. Lopez-Mendez A, Daniel WW, Reading JC, Ward JR, Alarcon GS. Radiographic assessment of disease progression in rheumatoid arthritis patients enrolled in the cooperative systematic disease program randomized clinical trial of methotrexate, auranofin or a combination of the two. Arthritis Rheum 1993; 36:1364-9.

33. Willkens RF. Comment to letter of R Madhok and N Menon. Arthritis Rheum 1993; 36:1183-4.

34. Willkens RF, Sharp JT, Stablein D, Marks C, Wortmann R. Comparison of azathioprine, methotrexate, and the combination of the two in the treatment of rheumatoid arthritis. A forty-eight-week controlled clinical trial with radiologic outcome assessment. Arthritis Rheum 1995; 38:1799-806.

35. Felson DT, Anderson JJ, Boers M, et al. American College of Rheumatology preliminary definition of improvement in rheumatoid arthritis. Arthritis Rheum 1995; 38:727-35.

36. Dougados M, Cantagrel A, Goupille P, et al. Sulfasalazine, methotrexate, and the combination in early rheumatoid arthritis: a double blind randomized study. Arthritis Rheum 1996; 39:S103.

37. Haagsma CJ, van Riel PLCM, de Rooij DJRAM, et al. Combination of methotrexate and sulphasalazine versus methotrexate alone. A randomised open clinical trial in rheumatoid arthritis patients resistant to sulphasalazine alone. Br J Rheumatol 1994; 33:1049-55. 
38. van der Heijde DMFM, van ' $t$ Hof MA, van Riel PLCM, et al. Judging disease activity in clinical practice in rheumatoid arthritis; a first step in the development of a disease activity score. Ann Rheum Dis 1990; 49:916-20.

39. Moreland LW, Kimberley RP, Alarcon GS. European and US rheumatologists agree in triple but not on double or single early DMARD choice for different types of RA. Arthritis Rheum 1997; 40:S218.

40. O'Dell J. Combination therapy for rheumatoid arthritis: apparent universal acceptance. Arthritis Rheum 1997; 40:S119.

41. Felson DT, Anderson JJ, Meenan RF. The efficacy and toxicity of combination therapy in rheumatoid arthritis. A meta-analysis. Arthritis Rheum 1994; 37:1487-91.

42. Edmonds JP, Scott DL, Furst DE, Brooks P, Paulus HE. Antirheumatic drugs: a proposed new classification. Arthritis Rheum 1993; 36:336-9. 


\section{Reply to comments on our systematic review on combination therapy in rheumatoid arthritis.}

Rheumatology 1999; 38: 789-90.

SIR, We thank Drs O'Dell, Moore and Klassen for their reaction to our systematic review on combined treatment with second-line anti-rheumatic drugs in rheumatoid arthritis [1]. This allows us to discuss their study [2] in more detail than was possible in our original review [3]. Any review contains arbitrary elements. Therefore, it is essential that all choices in the process of data aggregation be explicit. In this way, the final evaluation is up to the readers. With Dr O'Dell c.s. we recognize the importance of long-term follow-up. Considering the small number of studies with extended follow-up, we chose to compare data on outcomes from the selected high-quality studies after the most extended period of treatment, with (1) patients in the intervention group(s) still on their intended regimen and (2) before any selection of patients with regard to therapy efficacy or other criteria had taken place (intention-to-treat principle).

We agonized over the best interpretation of the results of O'Dell et al's study, but finally decided that we did not agree with their own assessment. The key difference in interpretation lies at the 9 month point. O'Dell et al made a legitimate but arbitrary choice to regard any patient not meeting the $50 \%$ Paulus criteria for improvement at 9 months as a therapy failure. Unfortunately, these patients were not followed up further and no data is available on them. Notably, until 9 months, a suboptimal response could be handled by increases in methotrexate dose, so that most drop-outs during this period would be for other causes, e.g. toxicity. In contrast, the majority of drop-outs at or after 9 months would be for lack of efficacy.

In essence, the procedure results in 2 studies, reported as one: (1) a 9 month randomised controlled trial with the $50 \%$ criterion as endpoint (overall results according to intention-to-treat, $P$ $=0.12$, reported in our review as trend); (2) a further follow-up study of the responders. This has the potential to magnify the (trend) differences at 9 months, e.g. when the mean level of Paulus response in other groups would be slightly below $50 \%$. In addition, after selection of responders at the 9-month time point, the distribution of prognostic factors in the three groups (other than treatment) cannot be assumed to be comparable in the way they were at baseline, immediately after randomisation. In our view, any comparison of the three groups in such a follow-up study should be viewed with caution, and certainly not be pooled with the primary results under (1).

To support O'Dell et al's conclusion that triple therapy is more effective than the other modalities tested, we would need 2-yr data of all randomised patients. 
For instance, we would have liked to see 'sensitivity analyses' of the full dataset (i.e. all randomised patients) using different cut-off points for response. This is pertinent in view of the proven greater discriminative capacity of the ACR20 criterion [4]. It would also be nice to know which patients actually started alternative therapy after they were judged to be treatment failures according to the trial definition. Also, survival analyses are more suited for 'one-off' events such as recurrence of cancer, and less for disease activity, which is essentially a continuous variable. Thus, an analysis of the area under the curve of response (or another index) of all randomised patients might have added valuable information.

With O'Dell, we do believe that their results indicate that responders on triple therapy are more likely to maintain response on follow-up than patients in the other groups.

In conclusion, the final interpretation depends on whether one accepts the $50 \%$ Paulus criterion as sole judge of efficacy and, in addition, whether one is willing to assume that the selection of patients for follow-up after 9 months based on response did not influence the distribution of prognostic factors in the treatment groups (other than treatment) present at baseline.

We thank Dr Hurst for his reaction [5]. Pharmacokinetic explanations of differences in efficacy of various drug combinations are outside the scope of our review. Nevertheless, we agree with Dr Hurst that investigators should try to understand benefits that are due to altered pharmacokinetics. On the other hand, we do not think that complete understanding is a 'conditio sine qua non' for testing combinations, let alone a review on combination therapy. A recent study shows there is no interaction between methotrexate and sulphasalazine with usual doses for treatment of rheumatoid arthritis [6]. With regard to an interaction between methotrexate and cyclosporin, data presented at the FDA Arthritis Advisory Panel Hearing show that although some metabolites of methotrexate are slightly increased, these are not likely to have any antiinflammatory activity.

\section{A.C. VERHOEVEN, M. BOERS, P. TUGWELL}

1. ODell J, Moore GF, Klassen LW. Combination therapy in rheumatoid arthritis: a comment. Rheumatol 1999; 38:577.

2. O'Dell J, Haire G, Erikson N, et al. Treatment of rheumatoid arthritis with methotrexate alone, sulfasalazine and hydroxychloroquine, or a combination of all three medications. N Engl J Med 1996; 334:1287-91.

3. Verhoeven AC, Boers M, Tugwell P. Combination therapy in rheumatoid arthritis: updated systematic review. Br J Rheumatol 1998; 37:612-9.

4. Felson DT, Anderson JJ, Lange ML, Wells G, LaValley MP. Should improvement in rheumatoid arthritis clinical trials be defined as fifty percent or seventy percent improvement in core set measures, rather than twenty percent? Arthritis Rheum 1998; 41:1564-70.

5. Hurst NP. Combination therapy in rheumatoid arthritis: a comment. Rheumatol 1999; 38:789.

6. Haagsma CJ, Russel FGM, Vree T, van Riel PLCM van de Putte LBA. Combination of methotrexate and sulphasalazine in patients with rheumatoid arthritis: Pharmacokinetic analysis and relationship to clinical response. Br J Cin Pharm 1996; 42:195-200. 


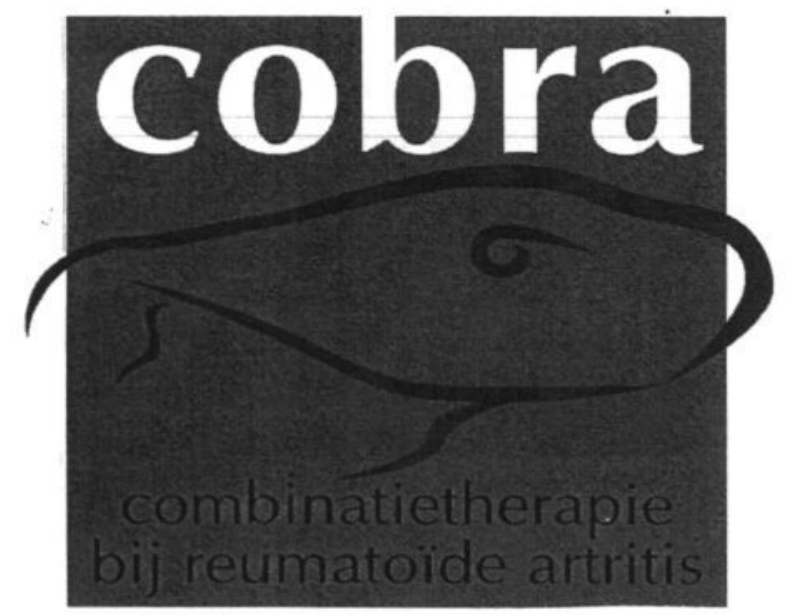




\section{CHAPTER 3}

\section{RANDOMISED COMPARISON OF}

COMBINED STEP-DOWN

PREDNISOLONE, METHOTREXATE AND

\section{SULPHASALAZINE WITH}

SULPHASALAZINE ALONE

\section{IN EARLY RHEUMATOID ARTHRITIS}

Maarten Boers, Arco Verhoeven, Harry Markusse, Mart van de Laar, René Westhovens, Christiaan van Denderen, Jendé van Zeben, Ben Dijkmans, André Peeters, Piet Jacobs, Hans van den Brink, Hubert Schouten, Désirée van der Heijde, Annelies Boonen, Sjef van der Linden

The Lancet 1997; 350: 309-18. 


\section{Randomised Comparison of Combined Step-Down \\ Prednisolone, Methotrexate and Sulphasalazine \\ with Sulphasalazine alone in Early Rheumatoid Arthritis}

\section{Abstract}

Background: The value of intensive combination therapy in early rheumatoid arthritis is unproven. In a multicentre, double-blind, randomised trial (COBRA), we compared the combination of sulphasalazine $(2 \mathrm{~g} /$ day), methotrexate $(7.5 \mathrm{mg} /$ week), and prednisolone (initially $60 \mathrm{mg} /$ day, tapered in 6 weekly steps to $7.5 \mathrm{mg} /$ day) with sulphasalazine alone.

Methods: 155 patients with early rheumatoid arthritis (median duration 4 months) were randomly assigned combined treatment (76) or sulphasalazine alone (79). Prednisolone and methotrexate were tapered and stopped after 28 and 40 weeks, respectively. The main outcomes were the pooled index (a weighted change score of five disease activity measures) and the Sharp/van der Heijde radiographic damage score in hands and feet. Independent health-care professionals assessed the main outcomes without knowledge of treatment allocation.

Findings: At week 28, the mean pooled index was 1.4 (95\% CI 1.2-1.6) in the combinedtreatment group and $0.8(0.6-1.0)$ in the sulphasalazine group $(P<0.0001)$. At this time, $55(72 \%)$ and $39(49 \%)$ patients, respectively, were improved according to the American College of Rheumatology criteria. The clinical difference between the groups decreased and was no longer significant after prednisolone was stopped, and there were no further changes after methotrexate was stopped. At 28 weeks, the radiographic damage score had increase by a median of 1 (range 0 $28)$ in the combined-treatment group and $4(0-44)$ in the sulphasalazine group $(P<0.0001)$. The increases at week $56(2[0-43]$ vs $6[0-54], P=0.004)$, and at week $80(4[0-80]$ vs 12 [0-72], $P=$ 0.01 ) were also significant. Further analysis suggests that combined therapy immediately suppressed damage progression, whereas sulphasalazine did less effectively and with a lag of 6 to 12 months. There were fewer withdrawals in the combined therapy than the sulphasalazine group (6 [8\%] vs $23[29 \%]$, and they occurred later.

Interpretation: This combined-therapy regimen offers additional disease control over and above that of sulphasalazine alone and persists for up to a year after corticosteroids are stopped. Although confirmatory studies and long-term follow-up are needed, this approach may prove useful in the treatment of early rheumatoid arthritis. 


\section{Introduction}

The treatment of rheumatoid arthritis (RA) is traditionally characterised by escalation. The first step is non-steroidal anti-inflammatory drugs (NSAIDs), and then if necessary a sequence of progressively toxic second-line drugs (disease-modifying antirheumatic drugs) is introduced [1]. There is evidence that some of these disease-modifying drugs provide a degree of disease control [2] - i.e. decrease disease activity but also maintain or improve physical function and retard radiographic joint damage [3]. However, both patients and physicians are dissatisfied with the long-term results of traditional therapy. A 1996 study suggested that early introduction of diseasemodifying drugs may be more beneficial than delayed introduction for patients with recently diagnosed RA [4]. Research is focused towards finding new, more effective drugs, reassessment and earlier use of existing drugs (such as corticosteroids [5]), and treatment with drug combinations [6].

The COBRA trial (COmbinatietherapie Bij Reumatoïde Artritis) is an adaptation of the latter two options - step-down bridge therapy with corticosteroids in early RA [1]. Our intention was to rapidly bring the disease under control in a very early stage, with agents that have overlapping windows of efficacy onset; and then, after 6 months to taper and stop the more toxic components while retaining disease control. We devised a regimen comprising a short period of high-dose oral prednisolone, rapidly tapered to a low maintenance dose. As the other components we chose methotrexate, commonly used as disease-modifying drugs of first choice in the USA, and sulphasalazine as the anchor drug to remain after the other two drugs were withdrawn. In Europe, sulphasalazine is commonly used as disease-modifying drugs of first choice.

We carried out a 56-week multicenter, randomised controlled trial among patients with early, active RA to study the degree of disease control afforded by a combination of sulphasalazine, methotrexate and high/low oral prednisolone given in the first 28 weeks, compared with that achieved with sulphasalazine alone; and to find out whether control could be maintained on sulphasalazine alone, after sequentially tapering and withdrawal of prednisolone and methotrexate in the second 28 weeks. 


\section{Patients and Methods}

\section{Patients}

We recruited patients between May, 1993, and May, 1995, in ten centres (nine in the Netherlands, one in Belgium). To optimise the benefit/risk ratio in line with the study purpose, we applied strict eligibility criteria to include patients with early RA who had very active disease and were most likely to benefit from this intensive treatment, in whom effects could be easily measured, and in whom we believed adverse effects would be least likely. The inclusion criteria were: a diagnosis of RA (American College of Rheumatology (ACR) criteria [7]) with onset of disease at or after 16 years of age; active disease of the joints and inadequate control of arthritis (due to lack of efficacy or toxicity of treatment); and treatment with NSAIDs in adequate doses for at least 3 months. Such treatment could already have been initiated at the start of symptoms, not necessarily at the time of diagnosis. We defined disease activity as the presence of six or more actively inflamed joints, located at three or more different sites (a site is defined as either one large joint or a group of small joints: the joints of the wrist, the metacarpophalangeals, the proximal hand interphalangeals, the distal hand interphalangeals, ankles, the tarsometatarsals, the metatarsophalangeals, and the proximal and distal foot interphalangeals) and presence of at least two of the following: nine or more tender joints (irrespective of site), morning stiffness of 45 minutes or longer, and a Westergren's erythrocyte sedimentation rate (ESR) of $28 \mathrm{~mm}$ or more in the first hour.

We excluded patients who had had RA for longer than 2 years, those previously or currently treated with any disease-modifying anti-rheumatic drug except anti-malarials (e.g., gold, Dpenicillamine, azathioprine, or cyclophosphamide) or corticosteroids (for arthritis or another disease), serious comorbidity or recent (within the 3 months before enrolment) major surgery, or inability to comply with the protocol. Adequate contraception was required. Further exclusion criteria were age below 18 or over 70; hypersensitivity to study medication, sulpha-containing compounds or aspirin; hypersensitivity to three or more drugs; active infectious disease; a history of tuberculosis, recurrent infections, recent ( $<3$ months) gastritis or gastrointestinal ulceration; any history of gastrointestinal bleeding or neoplasia; diabetes mellitus; hypertension treated with more than one antihypertensive drug; significant cardiovascular disease; liver disease; cataract; glaucoma; haematological disorders; partial or total colectomy; reduced renal function (creatinine clearance $<50 \mathrm{~mL} / \mathrm{h}$ ); proteinuria ( $>0.5 \mathrm{~g} /$ day); hypoalbuminemia; chronic dermatitis; treatment with phenytoin, phenylbutazone, salicylates, barbiturates, cholestyramine, probenecid, oral anticoagulants (dicoumarol derivatives); and a history of alcohol or substance abuse (i.e. inability to limit alcohol intake to a maximum of $70 \mathrm{~g}$ weekly) or use of any experimental drug less than 2 months before inclusion.

The study protocol was approved by research and medical ethics committees in all participating hospitals. The patients were fully informed about the potential side-effects of all the drugs. To maintain allocation concealment, they were told that response to treatment was variable in onset and efficacy with all three drugs. All patients gave written informed consent. 


\section{Intervention}

Both groups received sulphasalazine (Salazopyrine enteric-coated tablets of $500 \mathrm{mg}$, Pharmacia \& Upjohn, Uppsala, Sweden) $500 \mathrm{mg} /$ day, increased to $2000 \mathrm{mg} /$ day over a period of 3 weeks. In addition, the combination therapy group received prednisolone and methotrexate; the control group received matching placebo tablets and capsules identical in appearance and taste. The daily prednisolone dose was $60 \mathrm{mg}$ in week $1,40 \mathrm{mg}$ in week $2,25 \mathrm{mg}$ in week $3,20 \mathrm{mg}$ in week $4,15 \mathrm{mg}$ in week $5,10 \mathrm{mg}$ in week 6 , and $7.5 \mathrm{mg}$ thereafter (week 1-6 - one gelatine capsule containing the daily dose, capsule compound by Bufa, Uitgeest, Netherlands; week 7 and later - 5 $\mathrm{mg}$ tablets by CentraFarm Nederland bv, Etten-Leur, Netherlands; some of these tablets were broken by the pharmacy so that $7.5 \mathrm{mg}$ could be taken daily). The cumulative dose over the first 6 weeks was $1190 \mathrm{mg}$; over the first 28 weeks it was $2345 \mathrm{mg}$, corresponding to a mean of $12 \mathrm{mg}$ daily. The methotrexate prescription was $7.5 \mathrm{mg}$ in a single weekly dose (PharmaChemie bv, Haarlem, Netherlands). If an adverse event occurred, the drugs were temporarily withdrawn, and reintroduced at lower doses according to a fixed protocol where possible.

Prednisolone and methotrexate were stopped after 28 and 40 weeks, respectively. Both drugs were gradually withdrawn to decrease the chance of a disease flare. Thus, from week 29 to 35 , a day of zero prednisolone dose was introduced each week: first week, no prednisolone on Wednesday; second week, no prednisolone on Tuesday and Saturday; third week, no prednisolone on Monday, Wednesday, and Friday; until after 6 weeks, the prednisolone had been stopped. After 40 weeks of treatment, methotrexate was tapered: the drug was given at $5 \mathrm{mg}$ per week for 3 weeks, then at $2.5 \mathrm{mg}$ per week for 3 weeks, then stopped. If there was a flare in disease activity, the last medication stopped was reintroduced. A flare was defined per protocol as an increase of five in active joint count or an increase from zero to three compared to the situation at week 28 (an active joint is swollen or tender on pressure; counting of joint groups in one hand or foot as above). If the research medication had to be stopped for any reason and a consecutive diseasemodyfing antirheumatic drug was started, the protocol recommended that a drug not in the combination should be given, preferably intramuscular gold salts. After 56 weeks, the protocol ended, and the treating physician was at liberty to change second-line therapy, or to attempt a second tapering of methotrexate or prednisolone in those patients still on combination therapy. Where possible, blinded protocol treatment was continued. To maintain allocation concealment for other patients still in the protocol, the treatment code was revealed only for those patients still on combination therapy after 80 weeks.

\section{Concurrent therapy}

NSAIDs and simple analgesics were allowed; discontinuation was actively pursued. A maximum of two intra-articular steroid injections was allowed in two periods after week 28 of the protocol, but not in the 6-week period preceding independent assessment. Any other intervention with parenteral or oral corticosteroids was not permitted. All patients received folic acid (1 $\mathrm{mg} /$ day) during methotrexate or placebo prescription and elemental calcium $(1 \mathrm{~g} /$ day, carbonate composite) during prednisolone or placebo prescription. Vitamin D deficiency apparent at the laboratory screening before inclusion was corrected. 


\section{Treatment allocation}

Patients who met the eligibility criteria were entered into the study and assigned a unique study identification number by telephone. This number implied random allocation to one of the two treatments with stratification by centre. For each centre, a separate randomisation list was generated by an adaptive biased urn algorithm. In contrast to fixed blocks, this algorithm ensured that the rheumatologists would have no clue to the allocation of each subsequent patient in a setting where unblinding was possible; yet it also guaranteed an approximately equal distribution over the groups even in the centres with smaller numbers of patients [8]. The assignment was only known by the employees of the Maastricht Hospital pharmacy who prepackaged the medication; it was disclosed to the treating physician only in case of an emergency. Primary analysis was done with coded group allocation after entry of all study data. The full randomisation codes remained concealed until completion of the primary analysis.

\section{Organisation}

Each centre was staffed by a rheumatologist, a research nurse and an independent assessor. The rheumatologist was responsible for the identification and inclusion of the patients, and for all medical policy decisions. The research nurse monitored patient safety through regular follow-up schedules (first weekly, then every 4 weeks) and also measured disease activity. Independent assessors (mostly physiotherapists) applied the outcome measures at baseline and at week 16, 28, 40, and 56; in almost every instance a patient was seen by the same assessor. These health professionals were not involved in care of patients; they were also asked not to discuss disease activity or the treatment with the patients. Independent assessment ensured optimum concealment of primary outcome assessments, especially important in the first 6 weeks of the protocol, when potential effects and side-effects of high-dosed prednisolone would be most apparent. These assessments included all primary and core-set outcome measures except pain score, grip strength, and ESR. The follow-up schedule is continuing; all outcomes of the first 56 weeks are reported here. In response to criticism about the follow-up period for the radiographs, we read and analysed the 80 -week radiographs at a later stage.

Before the study and then once a year, all study personnel trained together to maintain assessment quality and agreement between observers. A specially designed manual of procedures and assessment techniques was available in each study centre. The trial was coordinated and data managed in the University Hospital Maastricht. Safety and toxicity were monitored by a safety committee of two independent rheumatologists, the Maastricht University pharmacist, and a statistician (HS). The pharmacy centre of the University Hospital Maastricht was responsible for drug production, packaging, and distribution.

\section{Assessment of endpoints}

The primary endpoint of the therapeutic intervention was a pooled index summarising the change in five measures after 28 weeks of treatment. Pooling is a validated method to increase sensitivity of separate measures [9]. To obtain the pooled index of one of the groups at week 28, we calculated a standardised change score of that group by dividing the mean change in one measure by its pooled standard deviation of change at week 28 . This procedure was repeated for 
each of the five measures; the pooled index is the mean of the standardised scores. To obtain pooled index values for another time point, change scores at that point were standardised through division by the same pooled standard deviation at week 28 . Finally, a constant was added to all index values so that the value at baseline was zero. We selected five measures for maximum sensitivity to change [10]: Tender joint count (68 joints [11); overall assessment by the independent assessor (on a $100 \mathrm{~mm}$ visual analogue scale, worst and best imaginable health status at the left and right anchor, respectively); grip strength (by vigorimetry; Martin, Tottlingen, Germany, range 0-150 kPa, mean of medians of three measurements in both hands [12]); ESR (Westergren method); and McMaster Toronto arthritis questionnaire [13], which follows improvement in five impaired activities elicited and priority-ranked by the patient during a baseline interview, together with change scores for quality of life, psychological, social and emotional wellbeing. The scores of this questionnaire reflect change, increase as disability improves, and vary from 10 (maximum deterioration) to 40 (maximum improvement). In its original format, the baseline questionnaire score differs from the follow-up scores because the change items are not available. To make these scores directly comparable, we added mock change items at baseline and scored them as "unchanged". Grip strength and ESR were assessed by the research nurses every week at the start of the protocol, later at least every 4 weeks.

We assessed all remaining disease activity measures of the World Health Organisation/ International League of Associations for Rheumatology core set as secondary endpoints [14]. As well was tender joint count, assessor's overall assessment, and acute phase reactant (ESR) included in the pooled index, these measures are swollen joint count (48 joints: modified from American College of Rheumatology 66-joint count [11]; small foot joints as one joint site and no midfoot joints), pain (assessed by patient on a $100 \mathrm{~mm}$ visual analogue scale; worst imaginable pain at the right anchor), and patient's global assessment (on a $100 \mathrm{~mm}$ visual analogue scale, worst and best imaginable health status at the left and right anchor, respectively). To facilitate comparison with other studies, the highly patient-specific MacMaster Toronto arthritis questionnaire was complemented with the more generic health assessment questionnaire (HAQ; Dutch validated version; scores 0-3, 3 indicating a poor functional state) [15].

We expressed improvement in individual patients by the American College of Rheumatology preliminary criteria for remission [16] (occurrence and duration; because no inquiry on fatigue was made we used the concept of a 'probable remission' for instances in which a patient would be in remission when absence of fatigue was assumed). Furthermore, we used the American College of Rheumatology preliminary criteria for improvement in RA [17] (i.e. minimum $20 \%$ improvement in tender and swollen joint counts plus a similar improvement in at least three of five remaining core-set measures). Before calculating improvement percentages, we ensured (by recoding if necessary) that all scales decreased on improvement. We also report improvement with application of a $50 \%$ threshold instead of $20 \%$ in the American College of Rheumatology criteria.

To facilitate comparison with other European studies, we report the disease activity score (DAS) [18], a composite outcome measure containing the Ritchie tender joint index (RI), swollen joint count (SJC), ESR and patient's overall assessment (POA);

$\mathrm{DAS}=0.54 \cdot \sqrt{\mathrm{RI}}+0.065 \cdot \mathrm{SJC}+0.33 \cdot \ln \mathrm{ESR}+0.007 \cdot \mathrm{POA}$. 
The term disease-controlling has been suggested for antirheumatic treatment regimens that improve disease activity, retain or improve physical function, and decrease progression of radiographic damage [3]. A priori, we expected our study to be too small to detect small differences in radiographic progression between the two groups, since both were treated with the disease-controlling drug sulphasalazine. Nevertheless, two trained observers (AB \& AV) assessed radiographic damage, unaware of the identity of the patients. They separately scored radiographs of hands and feet according to van der Heijde's modification of Sharp's method [2]. This method reflects erosions and joint-space narrowing in 44 joints in the hands and feet. The principal measure, the total score, is the sum of erosion and narrowing scores, and ranges from 0 to 448 . The method requires radiographs to be presented in ordered fashion (baseline, and weeks 28, 56 and 80). Scores can either be stable or increase; decrease (indicating improvement) is not possible. We report the mean of the two observers' erosion, narrowing, and total scores.

As an exploratory analysis, we also report the cumulative number of joints free of erosions at baseline in which at least one erosion developed during follow-up. For this purpose, joints were grouped into four areas on each side: wrist (six joints), metacarpophalangeal (five joints); proximal interphalangeal (four joints); and foot joints (six joints). The first erosion in each area was counted. Furthermore, we explored the rate of radiographic change in each of the measurement periods by calculating not only the change scores from baseline, but also the change scores between week 28 and 56 and between week 56 and 80 .

\section{Toxicity and monitoring}

Laboratory monitoring at every control visit comprised complete blood count, measurement of serum total bilirubin, aminotransferases, alkaline phosphatase, creatinine, blood urea nitrogen, and electrolytes, and urinalysis for glucose and albumin. Toxicity was assessed by counting each adverse event reported and possible subsequent changes to treatment (e.g. withdrawal). Each patient underwent pulmonary function tests (expiratory volume and carbon monoxide diffusion capacity) at baseline and twice yearly thereafter. Bone densitometry was done by an operator unaware of treatment assignment, in all centres where a dual-energy X-ray absorptiometer was available (Lunar, Hologic or Norland, in eight centres). We report changes in bone density for lumbar spine and femoral neck (mean of right and left hip).

We assessed IgM rheumatoid factor serostatus in a time-resolved fluoroimmunoassay (rabbit IgG antigen; Nordic, Tilburg, Netherlands); values over $20 \mathrm{kU} / \mathrm{L}$ classified patients as positive for rheumatoid factor [19]. Class II human leukocyte antigen (HLA) genotype was identified by serological typing (Tissue Typing Laboratory, Maastricht University Hospital). 


\section{Compliance}

We assessed compliance by tablet counts at every control visit, by questioning (including a quantification of the number of tablets missed), and by measurement of sulphapyridine (a sulphasalazine metabolite) in urine samples taken at weeks 16,28 , and 40 . We classified as noncompliant all patients on protocol treatment who were negative for sulphapyridine once or who failed to return tablet boxes at control visits more than once. In the first 28 weeks, we made judgements at every control visit, and classified as 'probably non-compliant' patients who in the first 28 weeks on average missed more than one daily dose a week of sulphasalazine or prednisolone, or more than one weekly dose of methotrexate over a period of 6 weeks.

\section{Analysis}

The target sample size was 168 patients. This number yields a power of at least $90 \%$ to detect a difference of 0.33 or greater in the pooled index (SD 0.45) between the treatment groups at twosided $\alpha=0.05$, given a maximum dropout rate of $50 \%$. Enrolment stopped at 156 patients for practical reasons, because the actual drop-out rate was $20 \%$. All analyses were based on intention to treat as initially assigned. All available data were used. Data missing due to loss to follow-up were handled by a last observation carried forward approach. For other missing data, values were interpolated if actual assessments were available at least every 28 weeks. No interim analyses were done.

Measures with a Gaussian distribution are expressed at baseline as mean and SD, and as mean change with $95 \%$ confidence intervals. The main endpoint was initially analysed by two-way ANOVA with treatment, centre and their interaction as factors. The latter two factors were not significant (centre $P=0.07$; interaction $P=0.79$ ). In view of the large effect of treatment, further analyses ignored centre as a factor, with exception of the multivariable analysis. Measures with a non-Gaussian distribution are expressed as median and median change (minimum-maximum) and analysed with Mann-Whitney tests; measures with a discrete distribution are expressed as counts (\%) and analysed by continuity-corrected $\chi^{2}$ tests or Fisher's exact tests where appropriate. The level of significance was set at $P<0.05$, two-sided. No adjustment was made for multiple testing.

For the main clinical and radiological outcome, multivariable analyses tested whether imbalance in important prognostic factors between the two groups remaining despite randomisation affected the study results. The dependent variables were the pooled index and the progression in total radiological damage score at 28 weeks. The latter was log-transformed (new variable $=\log$ [total change score +1$]$ because of a skewed distribution. Full and parsimonious models were constructed: the full models contained the predictive variables: treatment group, centre, sex, age, disease duration, rheumatoid factor status, presence of HLA genotype DR4 or DR2, number of years education, marital status, and baseline scores for DAS, HAQ, and radiological damage. The parsimonious models contained only those variables selected in forward stepwise regression analysis $(F$ to enter $>4.0$ ). Plots of residuals versus fitted and versus predictor values were inspected for departure of regression analysis assumptions. Because there was still substantial skewness after log transformation on the total radiological damage score, the results of the regression analysis on this variable must be interpreted with caution. 


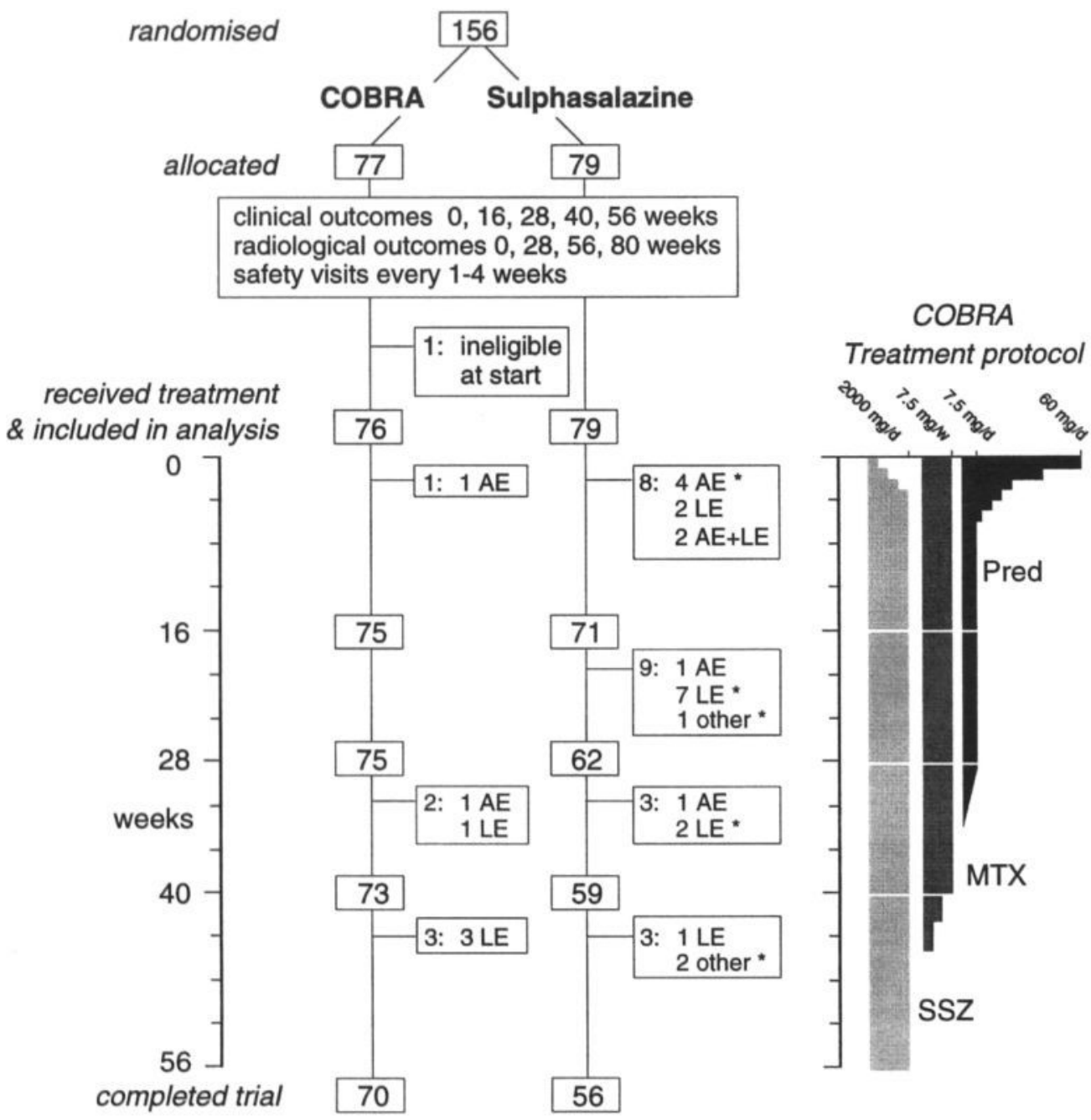

Figure 3.1: Trial profile.

* One patient lost to follow-up.

$\mathrm{AE}$, adverse event; $\mathrm{LE}$, loss of efficacy; 'other' includes protocol violations. 


\section{Results}

The trial included 156 patients (Figure 3.1). In one patient the protocol medication was stopped within 1 week because his disease was in spontaneous remission at baseline. Data of this patient are not reported. 76 patients received combination treatment, 79 sulphasalazine only. Five patients ( $3 \%$, all in the sulphasalazine group) were lost to follow-up before week 56 . In six patients (all withdrawn) the treatment assignment had to be revealed before week 56 for medical reasons.

The two treatment groups were similar in terms of baseline disease activity, radiographic damage, demographic and other prognostic variables (Table 3.1). Except for one Asian patient in each group, all patients were white. Our eligibility criteria resulted in a study group with very early, active, and severe RA; in $77 \%$ the trial medication comprised the first disease-modifying antirheumatic drug. High rates of rheumatoid-factor positivity, HLA-DR4 genotype, and presence of radiographic damage all indicate a poor a-priori prognosis (Table 3.1). In $21 \%$ of all patients (i.e. $32 \%$ of those with baseline erosive disease) erosions were initially found only in the feet.

Table 3.1: Baseline characteristics of study patients.

$$
\begin{array}{cc}
\text { Combined treatment } \\
(n=76)
\end{array} \quad \begin{gathered}
\text { Sulphasalazine } \\
(n=79)
\end{gathered}
$$

Demography

Age in years, mean (SD)

$49.5 \pm 11.9$

$49.4 \pm 12.3$

Female

Educational years, mean (SD)

$50 \quad(66 \%)$

$41 \quad(52 \%)$

$10.0 \pm 3.0$

$9.6 \pm 3.3$

Cinical characteristics

Disease duration in months, median (min-max)

$\begin{array}{rr}4 & (1-24) \\ 16 & (21 \%) \\ 9 & (12 \%) \\ 59 & (78 \%) \\ 44 & (60 \%) \\ 16 & (22 \%) \\ 55 & (74 \%)\end{array}$

4

19

Previous treatment with antimalarials

Hospitalized for RA at baseline

Positive IgM rheumatoid factor

HLA-DR4 positive *

HLA-DR2 positive *

Erosions on hand or foot radiographs ${ }^{+}$

$s^{+}$

* Human leukocyte antigen; homozygotic or heterozygotic DR type, assessed in 143 patients (92\%).

$\dagger$ Patients with available baseline radiographs; combined-treatment $n=74$, sulphasalazine group $n=75$. 


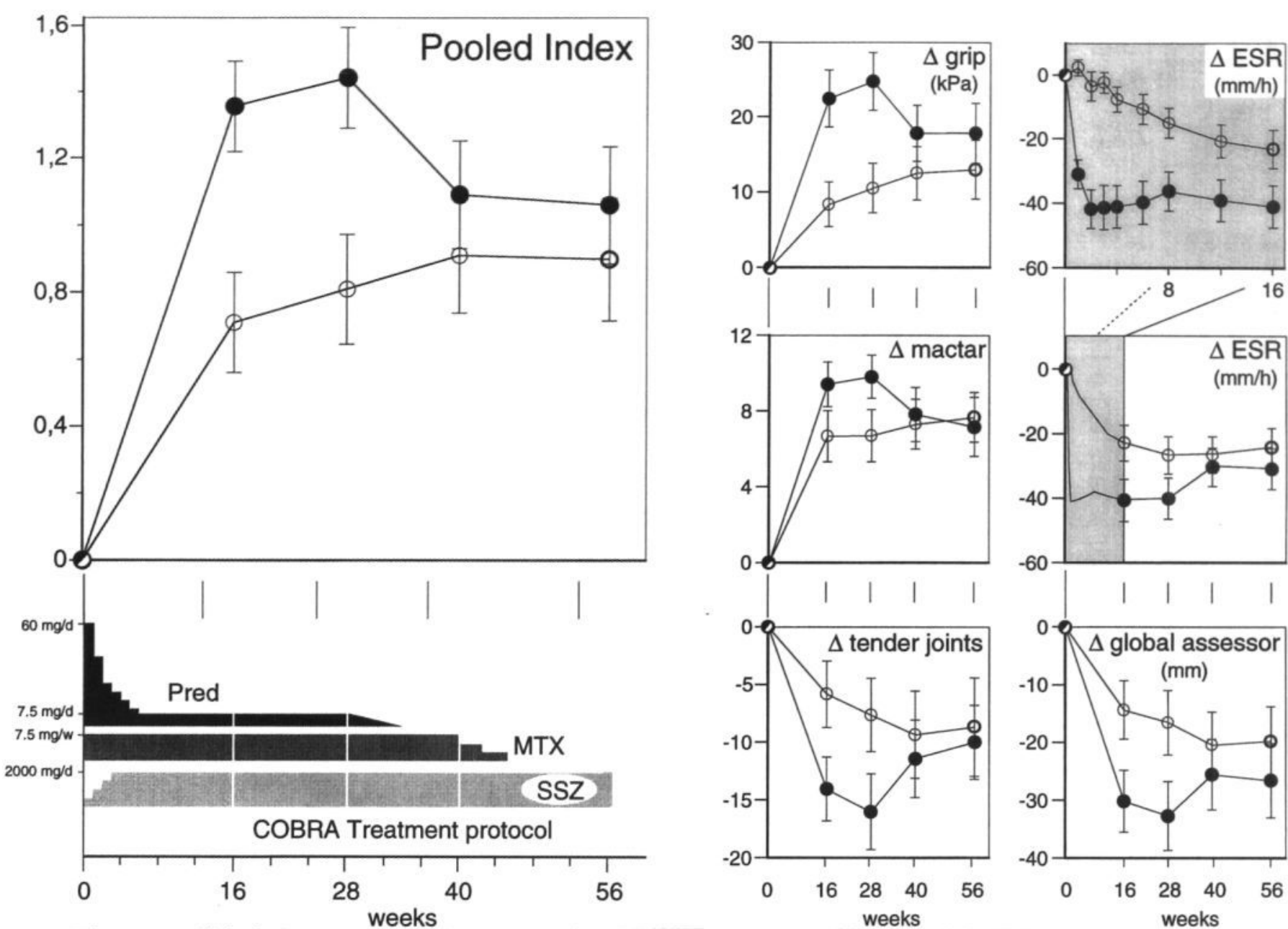

Figure 3.2: Cinical outcome of treatment, expressed as mean $(95 \% \mathrm{CI})$ pooled index and changes in its components parts.

Positive values indicate improvement in pooled index, grip strength, and MACTAR questionnaire, negative values in the remaining measures. Changes in ESR in first 16 weeks are shown in graph in upper right corner (note different time scale). 


\section{Clinical outcome}

Within a few weeks, combined therapy greatly improved disease activity in most patients (Figure 3.2, Table 3.2). Sulphasalazine also improved disease activity substantially, although less than combined treatment. There was an almost immediate response to combined treatment in all other frequently assessed measures (e.g. grip strength, pain, ESR, the latter shown in Figure 3.2). Despite a daily prednisolone dose of only $7.5 \mathrm{mg}$ from week 7 onwards, further improvement continued up to week 28. At this time, the clinical efficacy of combined treatment was almost double that of sulphasalazine (Figure 3.2, Table 3.2). 55 (72\%) of the combined-treatment group compared with $39(49 \%)$ of the sulphasalazine group had improved according to "20\% ACR" criteria $(P=0.006)$, and $37(49 \%)$ compared with $21(27 \%)$ had improved under the "50\%" criteria $(P=0.007)$.

In the combined-treatment group 16 patients had probable remissions and five definite remissions during the first 28 weeks (total $28 \%$ ). In the sulphasalazine group, the corresponding numbers were nine and four (total $16 \% ; P=0.14$ ). In the combined-treatment group only, remissions clustered near the beginning and end of the first 28-week period. Almost all of these ended when prednisolone was stopped, and in the second 28 weeks, only a few additional patients had remissions. For the total study period, there were 18 probable and six definite remissions $(32 \%)$ in the combined-treatment group, compared with 14 and five in the sulphasalazine group $(24 \% ; P=0.38)$. Of these, only one patient in the combined-treatment group and three in the sulphasalazine group had persisting remission at 56 weeks.

The difference in clinical efficacy between the treatment groups decreased and was no longer significant after withdrawal of prednisolone, and there were no further changes when methotrexate was withdrawn (Figure 3.2). However, differences in two of three measures of physical function (HAQ, grip strength) remained near significance (Table 3.2). Prednisolone was restarted (for disease flares) in six patients in the combined-treatment group. Methotrexate was restarted in 13 patients in the combined-treatment group, and methotrexate placebo in three patients in the sulphasalazine group.

Adjustment for prognostic variables did not change the difference in efficacy between treatments (crude coefficient for the additional effect of combined treatment $v$ sulphasalazine on the pooled index at week 28: 0.63 [SE 0.12], $P<0.0001$; adjusted coefficient 0.59 [0.11], $P<$ 0.0001 ). In stepwise regression analysis, baseline physical disability score and disease duration, as well as centre, significantly affected the pooled index at week 28 ; patients with high initial disability and shorter disease duration were more likely to improve. The effect of treatment was not changed in the model incorporating these factors. 
Table 3.2: Main clinical outcomes of treatment after 28 and 56 weeks of combined $(n=76)$ and sulphasalazine $(n=79)$ treatment

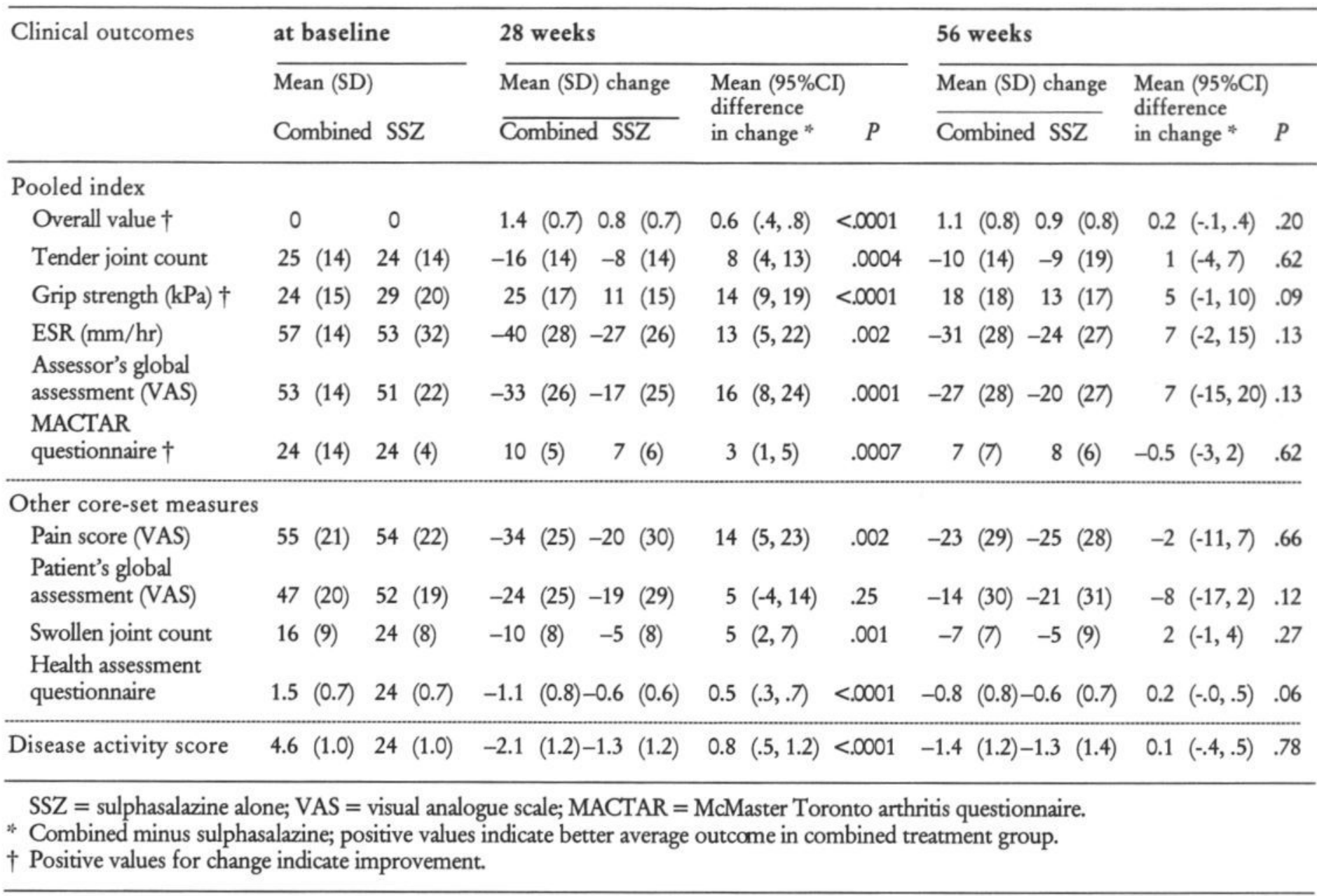




\section{Joint destruction on radiographs}

Radiographs of 147 patients (73 combined treatment, 74 sulphasalazine) were available for assessment (baseline and at least one follow-up). The between-observer reliability of the assigned total scores was satisfactory (within-class correlation coefficient of absolute scores 0.91 , and of change scores, 0.88 . Because of skewness in the data we also calculated Spearman rank correlations (absolute scores 0.90, change scores 0.89).

The groups were well balanced in terms of damage at baseline (Figure 3.3, Table 3.3). 26\% vs $22 \%$ of patients had no erosions; $23 \%$ vs $19 \%$ had a total score of 0 . The total score had increased significantly more in the sulphasalazine group than in the combined-treatment group at 28 weeks $(P<0.0001)$, 56 weeks $(P=0.004)$; and 80 weeks $(P=0.01$; Table 3.3, Figure 3.3$)$. The differences between the groups were greatest for increases in the erosion score (Table 3.3). At 28 weeks, $31 \%$ of the combined-treatment group vs $13 \%$ of the sulphasalazine group had stable scores $(P=$ $0.009)$; at 56 weeks, $19 \%$ vs $10 \%(P=0.23)$; at 80 weeks, $10 \%$ vs $7 \%(P=0.75)$. In both groups, patients without erosions at baseline showed little progression over 80 weeks: median increase 2 (0-25).

The findings on new erosive damage appearing in one of eight areas previously free of erosions $s$ were similar. After 28 weeks such damage had appeared in median zero areas (0-5) in the combined-treatment group, compared with one (0-6) in the sulphasalazine group $(P<0.0001)$. The corresponding numbers at 56 weeks were zero (0-6) and one $(0-7, P<0.0001)$, and at 80 weeks one (0-7) and two $(0-8, P=0.0004)$.

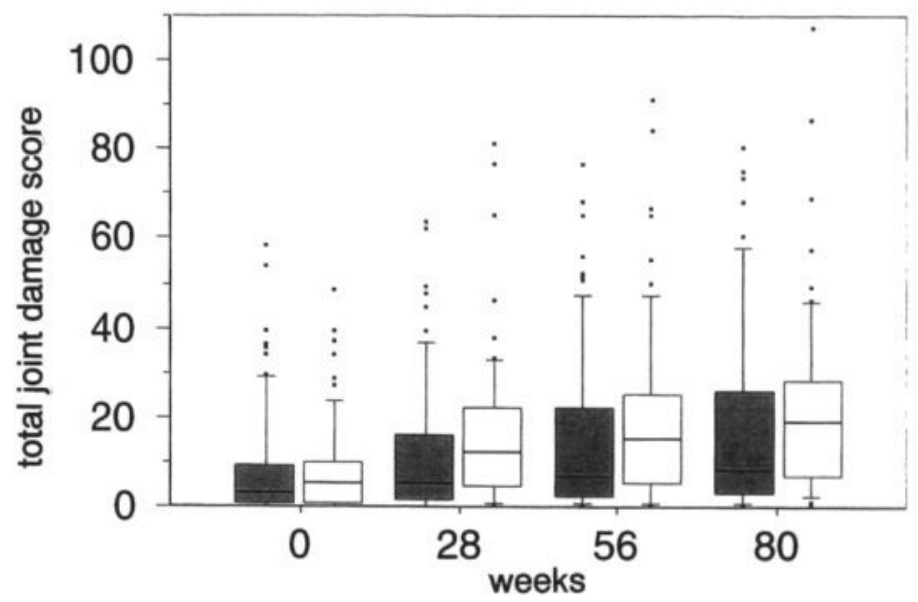

Figure 3.3: Effect of treatment on total radiographic damage score

Box-whisker plots of absolute radiographic damage scores (Sharp/van der Heijde method; summed total scores for erosions and joint-space narrowing in the hands and feet). Horizontal line in box is the median; limits of box are 25th and 75th percentiles; whiskers indicate 10th and 90th percentiles; values below and above these are plotted seperately. 
The damage rates calculated per study period showed slow progression of damage in the combined-treatment group in the first period (baseline to 28 weeks), whereas progression in the sulphasalazine group was more rapid (median increase in total score 0-28 weeks 1 [0-28] in the combined-treatment group vs 4 [0-44] in the sulphasalazine group; $P<0.0001$ ). In the second period, in which prednisolone and methotrexate were stopped (28-56 weeks), the rate of progression was again lower in the combined-treatment group than in the sulphasalazine group (median increase 1 [0-36] vs 2.5 [0-27], $P=0.04$ ), but during weeks 56-80 the rate of progression did not differ significantly $(1.5[0-36]$ vs $2.5[0-32], P=0.37)$.

Adjustment for prognostic variables did not change the difference in efficacy between treatments (crude coefficient for the additional effect of combined treatment $v$ sulphasalazine on the log-transformed total score at week $28-0.302$ [SE 0.075], $P<0.0001$; adjusted coefficient 0.297 [0.062], $P<0.0001$ ). In stepwise regression analysis, baseline values for radiological damage, DAS, HLA-DR4 genotype, and rheumatoid factor as well as centre significantly affected the progression of radiological damage at week 28. Patients who had higher baseline values for radiological damage and DAS and who were positive for HLA-DR4 and rheumatoid factor, had higher rates of progression. Again, the effect of treatment was not changed in the model incorporating these factors.

Table 3.3: Radiographic outcome of treatment; median scores (min-max) [2]

\begin{tabular}{|c|c|c|c|}
\hline & Combined treatment & Sulphasalazine & $P^{*}$ \\
\hline Baseline $^{\dagger}(n)$ & (73) & (74) & \\
\hline Erosion Score & $2 \quad(0-36)$ & $3 \quad(0-48)$ & \\
\hline Narrowing Score & $1 \quad(0-26)$ & $1 \quad(0-22)$ & \\
\hline Total Damage Score & $3 \quad(0-58)$ & $5 \quad(0-48)$ & \\
\hline 28 Weeks & $(71)$ & (71) & \\
\hline Erosion Score & $0 \quad(0-24)$ & $4 \quad(0-26)$ & $<0.0001$ \\
\hline Narrowing Score & $0 \quad(0-11)$ & $1 \quad(0-20)$ & 0.04 \\
\hline Total Damage Score & $1 \quad(0-28)$ & $4 \quad(0-44)$ & $<0.0001$ \\
\hline 56 Weeks & $(70)$ & $(65)$ & \\
\hline Erosion Score & $2 \quad(0-32)$ & $5 \quad(0-32)$ & 0.001 \\
\hline Narrowing Score & $1 \quad(0-28)$ & $1 \quad(0-30)$ & 0.53 \\
\hline Total Damage Score & $2 \quad(0-43)$ & $6 \quad(0-54)$ & 0.004 \\
\hline 80 Weeks & $(65)$ & $(56)$ & \\
\hline Erosion Score & $4 \quad(0-46)$ & $7 \quad(0-52)$ & 0.004 \\
\hline Narrowing Score & $2 \quad(0-35)$ & $2(0-34)$ & 0.40 \\
\hline Total Damage Score & $4 \quad(0-80)$ & $12(0-72)$ & 0.01 \\
\hline
\end{tabular}

* Mann-Whitney rank-sum test.

$\dagger$ Patients with baseline and at least one follow-up radiographic assessment. 


\section{Toxicity}

Significantly fewer patients stopped combined treatment than stopped sulphasalazine (Table 3.4, $P=0.0008$ ), and combination therapy patients dropped out later. Differences were apparent for both toxic effects and lack of efficacy. For instance, all four withdrawals from the combinedtreatment group because of lack of efficacy occurred after week 28, when prednisolone and methotrexate were stopped; by contrast, most of the 19 withdrawals for lack of efficacy in the sulphasalazine group occurred before week 28 (Figure 3.1). The adverse reactions that led to withdrawal of two patients in the combined-treatment group were gastrointestinal-tract complaints and dyspnoea (final diagnosis exacerbation of chronic bronchitis). In the sulphasalazine group the adverse reactions leading to withdrawal were rashes in four patients, gastrointestinal-tract complaints in two (one with concurrent proteinuria), granulopenia with a concurrent increase in aminotransferases in one patient, and thrombocytopenia (diagnosis preleukemic disease) in one.

The study medication was discontinued and restarted at an adjusted dose according to protocol in five patients. Three of these five patients (one in the combined-treatment group) had low granulocyte counts, the other two patients (both combined-treatment group) had high aminotransferases concentrations and gastrointestinal complaints, respectively. The remaining adverse events were not followed by withdrawal of study medication. These included 18 cases (12 combined treatment) of infection, treated as outpatients, 17 patients (nine combined treatment) with gastrointestinal complaints (no ulcer or bleeding); ten (six combined treatment) with cardiovascular disorders; including one myocardial infarction; and eleven (five combined treatment) with skin disorders. Various other complaints and transient laboratory abnormalities were reported in 37 cases ( 20 combined treatment).

Table 3.4: Reasons for withdrawal of patients from study

\begin{tabular}{|c|c|c|c|c|c|c|}
\hline & \multicolumn{3}{|c|}{ Combined treatment } & \multicolumn{3}{|c|}{ Sulphasalazine } \\
\hline & $n$ & $\begin{array}{l}\text { median time } \\
\text { (weeks) to } \\
\text { withdrawal }\end{array}$ & $(\min -\max )$ & $n$ & $\begin{array}{l}\text { median time } \\
\text { (weeks) to } \\
\text { withdrawal }\end{array}$ & $(\min -\max )$ \\
\hline \multicolumn{7}{|l|}{ Reason } \\
\hline Lack of efficacy & 4 & 42 & $(36-48)$ & 12 & 17 & $(16-40)$ \\
\hline Adverse reaction & 2 & 16 & (3-29) & 6 & 5 & $(2-31)$ \\
\hline Both & - & & & 2 & 9.5 & $(9-10)$ \\
\hline Other * & - & & & 2 & 28 & $(16-40)$ \\
\hline Protocol violation ${ }^{\dagger}$ & - & & & 1 & 50 & \\
\hline Total $\ddagger$ & 6 & 38.5 & $(3-48)$ & 23 & 16 & $(2-50)$ \\
\hline \multicolumn{7}{|c|}{$\begin{array}{l}\text { * One patient emigrated in week } 40 \text { of follow-up, one became pregnant. } \\
\dagger \text { Treated with parental corticosteroids for pulmonary disease. } \\
\ddagger \text { Difference in total number of withdrawals: } P=0.0008 \text {. }\end{array}$} \\
\hline
\end{tabular}


Expected adverse effects such as weight gain and hypertension were monitored at every control visit (i.e., at least every 4 weeks); osteoporosis was assessed by twice-yearly bone densitometry where possible (64 combined treatment, 62 sulphasalazine patients assessed). Patients in both the combined-treatment and sulphasalazine groups gained weight (mean gain at 28 weeks $2.5 \mathrm{~kg}[95 \% \mathrm{CI} 1.8,3.2]$ vs $0.7 \mathrm{~kg}[-0.2,2.2], P=0.002$; at 56 weeks $1.7 \mathrm{~kg}[0.8,2.6]$ vs $1.2 \mathrm{~kg}[0.2,2.2]$ respectively, $P=0.49)$. Blood pressure remained stable in both groups. Baseline characteristics and prognostic factors for osteoporosis were balanced and mean initial bone density was normal (1.134 [SD 0.19] g/ $\mathrm{cm}^{2}$ in lumbar spine and $0.920[0.14] \mathrm{g} / \mathrm{cm}^{2}$ in femoral neck). Eight women in the combined-treatment group and one in the sulphasalazine group were using hormone replacement therapy. During the first 28 weeks, the mean changes in lumbar bone density in the combined-treatment and sulphasalazine group $(n=64,62)$ were $-1.2 \%(-2.0,-0.3)$ and $0 \%(-0.9,0.9)(P=0.06)$. At 56 weeks, the changes were $-1.3 \%(-2.3,-0.4)$ and $-0.3 \%(-1.4$, $0.8)$ respectively $(P=0.15)$. In the femoral neck the corresponding bone density changes were -0.6 $(-2.1,0.9)$ vs $-0.7(-2.1,0.7)$ over 28 weeks, and $-1.9(-3.1,-0.7)$ vs $-1.3(-2.5,-0.1)$ over 56 weeks (both $P>0.2$ ). Eight $v$ six patients lost more than $5 \%$ (mean $8 \%$ ) of spinal bone; $14 \tau$ nine lost more than $5 \%$ (mean $8 \%$ ) of femoral neck bone. These losses typically occurred in the first six months, with stabilisation or improvement thereafter.

Lung function measurements showed no important changes during the first 56 weeks (data not shown).

\section{Compliance and protocol violations}

Of patients who completed the 56-week treatment protocol, nine (six combined treatment, three sulphasalazine) were classified as non-compliant - eight were negative for sulphapyridine in at least one of the urine samples taken at weeks 16,28 , and 40 ; and one did not return medication for pill counts more than once. Fifteen other patients (six combined treatment, nine sulphasalazine) were classified as probably non-compliant. Thus, compliance was deemed satisfactory in 131 patients (85\%). Eight patients received intra-articular injections with corticosteroids outside the permitted periods (two of them had been withdrawn; three were in the combined-treatment group, five in the sulphasalazine group). Eight patients in the sulphasalazine group started treatment with oral corticosteroids after they were withdrawn from the trial. In addition, one patient in the sulphasalazine group was withdrawn because she needed corticosteroids to treat pulmonary disease. 


\section{Discussion}

The combination of an extended oral pulse of corticosteroids, methotrexate, and sulphasalazine led to an immediate, substantial, and highly significant improvement of disease activity and physical function in most patients with severe, early RA. On a low daily maintenance dose of $7.5 \mathrm{mg}$ prednisolone from week 7 onwards, this improvement continued at a slower pace up to week 28 , and was almost double that of conventional treatment with sulphasalazine alone in all clinical measures. The study did not have sufficient power to show statistical significance for differences in remission rates. Combined therapy also had beneficial effects on joint damage as shown on hand and foot radiographs. This degree of clinical efficacy could not be maintained by sulphasalazine alone - most of the clinical differences between the groups decreased and were no longer significant after prednisolone was withdrawn, and there were no further changes after methotrexate was stopped. However, from inspection of an area under the curve we suggest that the patients who received combined treatment had major clinical benefit throughout the year. This view is supported by the low withdrawal rate for any reason during the study period. The benefits of combined therapy on radiography persisted up to week 80 . We believe, therefore, that combined therapy can be classified as a disease-controlling anti-rheumatic therapy [3].

The differences between COBRA and sulphasalazine are all the more striking because sulphasalazine alone also performed well as a disease-controlling anti-rheumatic drug - the onset of action was rapid, the withdrawal rate low (observed 29\%, expected $27-40 \%$ ), and the radiological progression rate was similar to that in another study in early RA [2]. The true clinical relevance of the effects of the combined treatment must be proven by follow-up studies. However, since demonstration of any effect on radiological progression in RA in difficult, we believe that the decrease, to a third of that of sulphasalazine - and possibly a sixth that of "symptom-modifying anti-rheumatic" drugs such as hydroxychloroquine - will prove clinically relevant.

Withdrawal rates for both toxicity and lack of efficacy were much lower with combined therapy than with sulphasalazine, and expected side-effects (especially of prednisolone such as osteoporosis) were of minor importance. The low withdrawal rate may by itself contribute to the differences in efficacy by preventing the loss of anti-rheumatic effect during the time when treatment is switched from one agent to another. There was a slight increase in infections with combined therapy but none led to (even temporary) protocol interruptions and all could be treated without hospital admission. Such toxicity figures must be interpreted with caution; with its small sample size and short follow-up, a clinical trial is not suited to reliably detection of side-effects that may be important. Given the limited period and dose of the combination, we do not expect relevant late morbidity. Nevertheless, the study cohort is being followed up to so that long-term benefits and risks of the therapy can be assessed. Thus we were able to read, analyse and report the 80 -week radiograph data ahead of time in response to criticism that 56 weeks of follow-up was too short. 
The randomisation procedure of our trial created prognostically similar treatment groups. Protocol violations, contamination and co-interventions were minor and did not affect the conclusions from intention-to-treat analysis. Compliance was satisfactory, in an admittedly crude assessment. However, concealment of allocation might raise some concerns. The treating rheumatologists responsible for recruitment (but not assessment) could potentially become aware of treatment allocation. However, the randomisation procedure guaranteed that they had no clue to the treatment allocation of subsequent patients. Outcome assessment was fully delegated to independent assessors: they were unaware of the rapid effects of corticosteroids because they were not involved in the care of these patients, and assessed them only at baseline and at weeks 16,28 , 40 , and 56 . They were asked not to discuss the disease or its treatment with the patients. Since they were health professionals but not physicians, they had less experience with corticosteroid sideeffects. The patients were fully informed about the potential side-effects of all the drugs. To protect against unblinding, they were told that response to treatment was variable in onset and efficacy with all three drugs. Although some unblinding due to large differences in efficacy cannot be ruled out, the effects of the subjective clinical measures were consistent, and reflected those in the objective measures (e.g. ESR).

Another concern that might be raised is the method of reading radiographs. To improve precision, these were read without knowledge of the patient's identity or group allocation, but they were ordered in sequence. This approach might be criticised as leading to bias. In general, opinions are divided on the value of having previous information available when a judgement is made. In the case of radiographs in studies of RA, and especially in early disease, random-sequence reading introduces a lot of noise, because small changes in positioning can temporarily hide erosions. In any case, both Larsen's original method [20] and the van der Heijde modification of Sharp's method [2] require sequential reading; these are the methods used in the majority of published studies (although some - e.g. Kirwan [5] - did score randomly). In our trial, any bias would work in the same direction in both groups, and would not alter the conclusions based on the differences between the groups.

The eligibility criteria in our trial selected RA patients with poor a-priori outlooks. For instance, a high proportion of patients had erosive disease at baseline (77\%), partly because we included of foot radiographs: on the basis of hand radiographs only the proportion of patients with erosive disease would have been $47 \%$. Nevertheless, some patients included may not have needed such an intensive approach as our combined treatment, since predictive variables never provide $100 \%$ accuracy [21]. On the other hand, such patients would probably show good clinical response, and withdrawal of prednisolone and methotrexate would be possible; thus the risks involved in their overtreatment are limited. We arbitrary excluded patients older than 70 , and further study is needed before this therapy can be advised to patients over 70, or for patients with a longer duration of RA or concurrent disease. Thus, our results apply to otherwise healthy patients with early and active RA treated in a specialist setting. The opportunity to intervene early with second-line antirheumatic drugs relies heavily on the early diagnosis of RA and rapid referral of patients; health-care systems should be specifically organised to facilitate this process. 
Combination therapy can be applied in many different ways. We chose a step-down strategy with rapidly acting drugs. This approach optimised the chance of efficacy in a potentially limited window of opportunity. Our results supports the view that corticosteroids are among the most effective and rapidly working disease-controlling antirheumatic drugs. A meta-analysis has confirmed that low-dose corticosteroids can be beneficial [22]. The toxic effects of corticosteroids may be comparable to those of some other disease-modifying anti-rheumatic drugs and even NSAIDs; and their use in established RA is widespread. However, doubts over the longevity of the effects, and fears of cumulative morbidity have limited their use in early RA. The optimal dosing schedule is also unclear.

In 1959 a trial of prednisolone at daily doses of $10-20 \mathrm{mg}$ indicated anti-rheumatic properties (both clinical and radiological), but with substantial side-effects [24]. Kirwan and colleagues [5] showed an effect of $7.5 \mathrm{mg}$ prednisone daily on the development of radiological damage together with only temporary effect on disease activity measures, and no side-effects. These investigators have since reported that joint destruction recommences after corticosteroids are withdrawn [25]. By contrast, the effect of our combined treatment on the progression of joint destruction persisted up to 1 year after corticosteroids were stopped. The initially more rapid progression in the sulphasalazine group slowed and approached that of the combined-treatment group with a lag of 1 year. These differences between our study and that of Kirwan might the result of more effective treatment not only in our experimental group but also our control group.

Van Schaardenburg and colleagues [26] found only temporary clinical effects of a prednisone regime starting with $15 \mathrm{mg}$ daily. Other studies with step-down strategies and oral or parental steroid pulses showed limited benefit and troubling rebound effects [27-29]. The limited clinical efficacy of corticosteroids in other trials may be due to the use of doses inadequate to bring the disease under control from the outset. Our results support the concept of step-down bridge therapy, and suggest that immediate and intensive suppression of high damage progression rates by a rapidly acting regimen may be sustained by another regimen with a slower mode of onset (e.g., sulphasalazine).

$60 \mathrm{mg}$ of prednisolone is a high daily dose for RA. However, the rapid tapering schedule resulted in a mean daily dose of $12 \mathrm{mg}$ overall, and $7.5 \mathrm{mg}$ daily from week 7 onwards. Also, the initial dose is not high compared with standard (but not always proven) therapy for most other severe autoimmune diseases (e.g., myositis, vasculitis, lupus nephritis) and is much lower than the intravenous methylprednisolone pulses that have been tried with limited success for many indications, including RA. The tapering schedule after week 28 proved to be too rapid, resulting in partial loss of benefit and some disease flares. This factor may have contributed to the relatively low numbers of lasting remission. We look forward to further studies with slower tapering schedules. 
We chose methotrexate as second drug in the combination because the onset of effect is rapid. However, in this and another trial, the response to sulphasalazine was as rapid as that to methotrexate [30]. In the design phase, we set a fixed, low dose of methotrexate because little was known about the toxicity of our combination. We would, with the data in available, now probably try dose intensification of methotrexate or sulphasalazine in case of suboptimum response (with the risk of additional toxic effects). The value of the combination of methotrexate and sulphasalazine is uncertain [30-32]. In our study, tapering of methotrexate had little impact on the mean results, although some patients had disease flares, which might be attributable to the withdrawal of prednisolone in the preceding period. The contribution of $7.5 \mathrm{mg}$ methotrexate weekly to efficacy and toxicity was probably relatively small in the second 6 months of treatment. However, whether the efficacy of the full combination in the first 6 months can be equalled by prednisolone/sulphasalazine or prednisolone/methotrexate combinations remains to be seen. In view of the high benefit-risk ratio of our combined treatment in the first 6 months, we believe maintenance of this ratio in the subsequent period is more desirable than reduction of the already low risk in the first 6 months.

To date, most drug combination trials have not shown addition or synergy, (i.e. benefits equal or better than the sum of benefits attributed to the single drugs). At best, investigators showed some enhanced efficacy at the expense of some extra toxic effects. However, Tugwell and colleagues [33] found that patients with long-standing RA who had only a partial response to methotrexate show clinically important improvement when cyclosporin was added to their regimen. O'Dell and colleagues achieved 50\% improvement in composite symptoms of arthritis with methotrexate, sulphasalazine and hydroxychloroquine [34]. $80 \%$ and $50 \%$, respectively, of the patients in these two studies were on low-dose corticosteroid maintenance therapy. Both studies show that substantial improvement is possible in established disease, and that more study on the merits of combination therapy is necessary.

Our study supports the view that corticosteroids in the proper regimen are among the most powerful disease-controlling anti-rheumatic drugs available. Early and intensive intervention in RA with this combination step-down schedule offers additional disease control over and above that of sulphasalazine alone. Damage control persists up to 1 year after corticosteroids are stopped, but to maintain optimum clinical efficacy after 28 weeks, another tapering schedule is probably necessary. 


\section{References}

1. Harris ED Jr. Management of rheumatoid arthritis. In: Kelley WN, Harris ED, Ruddy S, Sledge CB, eds. Textbook of rheumatology. Philadelphia: WB Saunders, 1993; 912-23.

2. van der Heijde DMFM, van Riel PL, Nuver-Zwart IH, Gribnau FW, van de Putte LBA. Effects of hydroxychloroquine and sulphasalazine on progression of joint damage in rheumatoid arthritis. Lancet 1989; i:1036-8.

3. Edmonds JP, Scott DL, Furst DE, Brooks P, Paulus HE. Antirheumatic drugs: a proposed new classification. Arthritis Rheum 1993; 36:336-9.

4. van der Heide A, Jacobs JWG, Bijlsma JWJ, et al. The effectiveness of early treatment with 'secondline' antirheumatic drugs, a randomized, controlled trial. Ann Intern Med 1996; 124:699-707.

5. Kirwan JR. The effect of glucocorticoids on joint destruction in rheumatoid arthritis. The Arthritis and Rheumatism Council Low-dose Glucocorticoid Study Group. N Engl J Med 1995; 333:142-6.

6. Tugwell P, Boers M. Long acting drug combinations in rheumatoid arthritis: updated overview. In: Wolfe F, Pincus T, eds. Rheumatoid arthritis: pathogenesis, assessment, outcome, and treatment. New York: Marcel Dekker, 1994:357-71.

7. Arnett FC, Edworthy SM, Bloch DA, et al. The American Rheumatism Association revised criteria for the classification of rheumatoid arthritis. Arthritis Rheum 1988; 31:315-24.

8. Schouten HJA. Adaptive biased urn randomization in small strata when blinding is impossible. Biometrics 1995; 51:1529-35.

9. Smythe HA, Helewa A, Goldsmith $\mathrm{CH}$. Independent assessor' and 'pooled index' as techniques for measuring treatment effects in rheumatoid arthritis. J Rheumatol 1977; 4:144-52.

10. Anderson JJ, Felson DT, Meenan RF, Williams HJ. Which traditional measures should be used in rheumatoid arthritis clinical trials? Arthritis Rheum 1989; 32:1093-9.

11. The cooperating clinics committee of the American Rheumatism Association. A seven-day variability study of 499 patients with peripheral rheumatoid arthritis. Arthritis Rheum 1965; 8:302-34.

12. Jones E, HanlyJG, Mooney R et al. Strength and function in the normal and rheumatoid hand. J Rheumatol 1991; 18:1313-8.

13. Tugwell P, Bombardier C, Buchanan WW, Goldsmith CH, Grace E, Hanna B. The MACTAR patient preference disability questionnaire: an individualized functional priority approach for assessing improvement in physical disability in clinical trials in rheumatoid arthritis. J Rheumatol 1987; 14:446-51.

14. Boers M, Tugwell P, Felson DT, et al. World Health Organisation and International League of Associations for Rheumatology core endpoints for symptom modifying antirheumatic drugs in rheumatoid arthritis clinical trials. J Rheumatol 1994; 41 suppl:86-9.

15. Siegert CEH, Vleming LJ, Vanderbroucke JP, Cats A. Measurement of disability in Dutch rheumatoid arthritis patients. Cin Rheumatol 1984; 3:305-9.

16. Pinals RS, Masi AT, Larsen RA. Preliminary criteria for clinical remission in rheumatoid arthritis. Arthritis Rheum 1981; 24:1308-15.

17. Felson DT, Anderson JJ, Boers M, et al. American College of Rheumatology preliminary definition of improvement in rheumatoid arthritis. Arthritis Rheum 1995; 38:727-35.

18. van der Heijde DMFM, van ' $t$ Hof MA, van Riel PLCM, et al. Judging disease activity in clinical practice in rheumatoid arthritis: first step in the development of a disease activity score. Ann Rheum Dis 1990; 49:916-20.

19. van der Sluys Veer G, Soons JWPH. A time-resolved fluoroimmunoassay of the IgM-rheumatoid factor. Eur J Cin Chem Cin Biochem 1992; 30:301-5.

20. Larsen A, Dale K, Eck M. Radiographic evaluation of rheumatoid arthritis and related conditions by standard reference films. Acta Radiol 1977; 18:481-91. 
21. van Zeben D, Hazes JMW, Z winderman AH, Vandenbroucke JP, Breedveld FC. Factors predicting outcome of rheumatoid arthritis: results of a follow-up study. J Rheumatol 1993: 20:1288-96.

22. Saag KG, Criswell LA, Sems KM, Nettleman MD, Kolluri S. Low-dose corticosteroids in rheumatoid arthritis. Arthritis Rheum 1996; 39:1818-25.

23. Fries JF, Williams CA, Ramey D, Bloch DA. The relative toxicity of disease-modifying antirheumatic drugs. Arthritis Rheum 1993; 36: 297-306.

24. Joint committee of the Medical Research Council and Nuffield Foundation. A comparison of prednisolone with aspirin or other analgesics in the treatment of rheumatoid arthritis. Ann Rheum Dis 1959; 18:173-87.

25. Kirwan JR, Hickling P, Jacoby R. Joint destruction recommences after glucocorticoids are withdrawn in early rheumatoid arthritis. Arthritis Rheum 1996; 39 suppl:S218.

26. van Schaardenburg D, Valkema R, Dijkmans BAC, et al. Prednisone treatment of elderly-onset rheumatoid arthritis; disease activity and bone mass in comparison with chloroquine treatment. Arthritis Rheum 1995; 38:334-42.

27. Giconelli RM, Ferraz MB, Visioni RA, Oliviera, Atra E. A randomized double-blind controlled trial of sulphasalazine combined with pulses of methylprednisolone or placebo in the treatment of rheumatoid arthritis. BrJ Rheumatol 1996; 35:150-4.

28. van Gestel AM, Laan RFJM, Haagsma CJ, et al. Oral steroids as bridge therapy in RA patients starting with parental gold. A randomized double blind placebo controlled trial. Br J Rheumatol 1995; 34:347-51.

29. Weusten BLAM, Jacobs JWG, Bijlsma JWJ. Corticosteroid pulse therapy in active rheumatoid arthritis. Semin Arthritis Rheum 1993; 23:183-92.

30. Haagsma C, van de Putte L, van Riel P. Sulfasalazine, methotrexate and combination in early rheumatoid arthritis, a double blind randomized study. Arthritis Rheum 1995; 38 suppl:S368.

31. Haagsma CJ, van Riel PLCM, de Rooij DJRAM, et al. Combination of methotrexate and sulphasalazine versus methotrexate alone. A randomised open clinical trial in rheumatoid arthritis patients resistant to sulphasalazine therapy. Br J Rheumatol 1994; 33:1049-55.

32. Dougados M, Cantagrel A, Goupille $\mathrm{P}$, et al. Sulfasalazine, methotrexate, and the combination in early rheumatoid arthritis: a double blind randomized study. Arthritis Rheum 1996; 39 suppl:S103.

33. Tugwell P, Pincus T, Yocum D, et al. Combination with cyclosporine and methotrexate in severe rheumatoid arthritis. NEngl J Med 1995; 333:137-41.

34. O'Dell JR, Haire CE, Erikson N, et al. Treatment of rheumatoid arthritis with methotrexate alone, sulfasalazine and hydroxychloroquine, or a combination of all three medications. NEngl J Med 1996; $334: 1287-91$. 


\section{CHAPTER 4}

\section{COST-EFFECTIVENESS AND \\ COST-UTILITY OF COMBINATION \\ THERAPY IN EARLY RA:}

RANDOMISED COMPARISON OF

COMBINED STEP-DOWN

PREDNisolone, Methotrexate

AND SULPHASALAZINE WITH

SULPHASALAZINE ALONE

Arco Verhoeven, Jack Bibo, Maarten Boers, Gerard Engel and Sjef van der Linden for the COBRA trial group

British Journal of Rheumatology 1998; 37: 1102-9. 


\section{Cost-Effectiveness and Cost-Utility of Combination Therapy in Early Rheumatoid Arthritis: Randomised Comparison of Combined Step-Down Prednisolone, Methotrexate and Sulphasalazine with Sulphasalazine Alone}

\section{Abstract}

Objective: Assessment of the cost-effectiveness and cost-utility of early intervention in rheumatoid arthritis (RA) patients, with combined step-down prednisolone, methotrexate, and sulphasalazine, compared to sulphasalazine alone.

Methods: Multicentre 56 week randomized double-blind trial with full economic analysis of direct costs and utility analysis with rating scale and standard gamble measurement techniques.

Results: The combined-treatment group included 76 and the sulphasalazine group 78 patients. The mean total costs per patient in the first 56 weeks of follow-up were $\$ 5519$ for combined treatment and $\$ 6511$ for treatment with sulphasalazine alone $(P=0.37)$. Out-patient care, inpatient care and non-health care each contributed about one-third to the total costs. The combined-treatment group appeared to generate savings in length of hospital stay for RA, nonprotocol drugs and costs of home help, but comparisons were not statistically significant. Protocol drugs and monitoring were slightly more expensive in the combined-treatment group. Clinical, radiographic and functional outcomes significantly favoured combined treatment at week 28 (radiography also at week 56). Utility scores also favoured combined treatment.

Conclusion: Combined treatment is cost-effective due to enhanced efficacy at lower or equal direct costs.

\section{Introduction}

Rheumatoid arthritis (RA) is a systemic disease with symmetrical inflammation of joints in upper and lower extremities as most important feature. Patients suffer from pain, stiffness, impaired function in daily life and at work, increased dependence on family and friends, and decreased participation in leisure activities. RA is associated with morbidity, worsening of quality of life and mortality $[1,2]$.

The monetary cost of RA is high due to increased use of out-patient medical services, increased hospitalization rates and major work disability in the course of the disease [3]. Intervention studies that include an economic evaluation in RA are rare [4] and, to our knowledge, nonexistent in early RA.

A combination therapy regimen of step-down prednisolone, methotrexate, and sulphasalazine, tested against sulphasalazine alone in early RA patients, demonstrated excellent clinical response, low toxicity and slowing of radiographic progression [5] (COBRA trial; OOmbinatietherapie Bij Reumatoïde Artritis). The current full economical analysis addresses the question whether this combined treatment is also cost-effective. 


\section{Patients and methods}

\section{Study design}

The methods of the COBRA study have been reported extensively [5]. Briefly, in a 1-year double-blind randomized clinical trial, $156 \mathrm{RA}$ patients (ACR criteria [6]) aged 18-70 yr, were randomly assigned to two treatment groups. All patients had early, active disease. No prior treatment with second-line anti-rheumatic medication, apart from antimalarials, was allowed. One group was treated with a combination of sulphasalazine, methotrexate and prednisolone; the other group was treated with sulphasalazine and double placebo. Prednisolone and methotrexate (or the placebos) were tapered and stopped after week 28 and week 40, respectively, while sulphasalazine was continued. All patients had calcium supplementation $(1 \mathrm{~g} /$ day) prescribed for as long as they used prednisolone, and folic acid ( $1 \mathrm{mg} /$ day) for as long as they used methotrexate. The primary clinical outcome was a pooled index [7]; a composite measure that reflects each patient's clinical improvement. This measure is suited for the comparison of clinical benefits between groups. In this report, the benefits are also expressed in terms of improved physical function and utility. All utility assessments were performed by trained independent assessors who contacted the patients only at baseline and four times thereafter. This way, the assessors stayed blind for effects of highdose prednisolone during the first 6 weeks of the protocol. The study protocol was approved by research and medical ethics committees in all participating hospitals (nine clinical centres in The Netherlands and one in Belgium). All patients gave informed consent in writing before they entered the study protocol between May 1993 and May 1995.

Costs

Costs were elicited from a societal perspective. To evaluate the economic consequences of combined treatment, only direct costs were considered. Direct costs are costs that are directly related to the intervention. These are detailed below in 5 parts: (1) costs of the intervention (protocol drugs and monitoring), (2) costs of non-protocol drugs, (3) other costs of out-patient care, (4) costs of in-patient care, (5) direct non-medical costs. The period of follow-up comprised 56 weeks in all patients.

Costs were primarily expressed in 1995 Dutch guilders and subsequently converted into US dollars (\$) at the 1994 Purchasing Power Parities rate of $2.143: 1$ [8]. Where possible, specific cost prices were derived from cost research performed in the University Hospital Maastricht (Financial Control Group internal report, February 1996). These cost prices were generalized to all participating clinical centres. Data on health care utilization were obtained from patient diaries specifically developed for this study. Patients were asked to complete forms for 56 weeks; these weekly forms were collected at every control visit. Additional data was collected from hospital records and biannual structured interviews. In case of doubt, the hospital record was decisive (e.g. to determine the exact duration of hospitalization). Patients were asked to report every health care utilization, regardless of whether it was related to their disease, because the symptoms and signs as well as the side-effects of treatment in RA are very heterogeneous, and possibly unknown [9]. 
Total intervention costs comprise costs of the therapeutic intervention itself and costs related to the necessary monitoring of adverse effects. The costs of protocol medication were calculated per patient by multiplying prices ( 1995 Dutch pharmacy standards, handling costs included) by volume of protocol medication in the first 56 weeks. The costs of calcium and folic acid supplements were only attributed to the combined-treatment group, although patients in both treatment groups had these prescribed. The costs of monitoring were based on a post hoc consensus among the trial rheumatologists who were knowledgeable on the prevalence of toxicity that had occurred during the trial. They set the frequency of out-patient rheumatology consultation at seven in the first year for either treatment, the frequency of laboratory monitoring for sulphasalazine treatment at seven and that for combined treatment at eight. With respect to the extensiveness of laboratory monitoring, we referred to the program that was used during the trial. This is open to discussion, and will be dealt with in the sensitivity analysis. Annual radiography of hands and feet to assess joint damage was considered to be part of normal clinical practice in patients with early RA and the integral monitoring schedule of both treatment groups. These costs were included. In addition, bone density measurement and thorax radiography were considered mandatory in patients before starting combined treatment to assess preexisting osteopenia and to exclude pulmonary tuberculosis; these costs were attributed to combined treatment only. Remaining costs strictly related to the execution of the trial were not included; e.g. costs of outcome assessments and extra visits.

Costs of non-protocol medication (prescription and 'over the counter' drugs) were assessed by records from the diaries. Four different classes of medication were distinguished: Non-steroidal anti-inflammatory drugs (NSAIDs) and analgetics, gastroprotective agents, disease-modifying antirheumatic drags (DMARDs) (apart from protocol medication) and miscellaneous.

Apart from medication, health care utilization included visits to the general practitioner, specialists, physiotherapists, and any diagnostic and all therapeutic procedures ordered as a consequence of these visits. The costs of consultation of a general practitioner (\$15) or physiotherapist (\$16) were based on charges. The costs of out-patient department specialist consultations (ranging from $\$ 22$ to 55 per consultation) were based on records of $26(17 \%)$ trial patients in the University Hospital Maastricht and generalized per treatment group to patients from other participating clinical centres. Although registered, costs for any aids (protheses and ortheses) and adjustments to the patients' homes are not reported here (the volume of different aids and adjustments is relatively small, and the reimbursement systems both in The Netherlands and Belgium vary substantially by municipality).

Costs of hospitalization were priced at $\$ 400 /$ day in a university or non-university hospital (based on cost-research) and $\$ 110 /$ day in a rehabilitation center (charge derived from the literature [10]). 
The direct non-medical costs comprise costs for professional or non-professional help at home, costs related to loss of leisure time of the patient and an accompanying person where necessary, transportation costs, and out-of-pocket expenses for disease-related activities and purchases. Costs of paid and unpaid help were derived from the time estimates registered in the weekly patient diary. The price for qualified house keeping was set at \$11. Unpaid help was valued at $80 \%$ of the price for professional help. Loss of leisure time due to visiting health care-providing institutes was valued at $\$ 2.12 /$ hour, a price derived from a literature survey [10]. Travel costs were calculated from the number of visits (excluding those for trial purposes only) and costs per visit. Kilometre distances were gathered from a route-planning computer package that uses postal area codes. The kilometre price was set at $\$ 0.27$, an amount also in use for reimbursement purposes. Out-of-pocket expenses include such costs as swimming in extra heated pools and costs of alternative health care (calculated from patients' reports in the biannual interviews).

As the time frame of follow-up in this report is only little over $1 \mathrm{yr}$, no discounting of future costs (or benefits) back to current value was carried through. Arthritis-related indirect costs, such as sick leave or occupational disability, are not reported here, but will be elucidated in a separate article with a follow-up period of $>1 \mathrm{yr}$.

\section{Efficacy measures; clinical effects}

The primarily clinical outcome was a pooled index. This composite measure comprised each patient's standardized improvement in erythrocyte sedimentation rate (ESR), grip strength, tender joint count, observer's global assessment and improvement in functional ability (scored from the MacMaster Toronto Arthritis patient preference interview [11]. A secondary endpoint was radiographic damage of joints in hands and feet, expressed in a quantitative score for erosions and joint-space narrowing [12]. Many clinicians feel that this type of radiological damage score represents cumulative disease activity [13]. Radiographs were read by two trained independent observers unaware of treatment allocation. Physical function was evaluated by a validated Dutch version of the Health Assessment Questionnaire $[14,15]$ filled out by the patients at baseline, and at weeks $16,28,40$, and 56 .

Interpolation of each patient's scores at five time points yielded a time-integrated score reflecting average clinical benefit and disability during the 56 week follow-up period. Direct costs in both treatment groups were related to the clinical benefits; between-group differences in timeintegrated units of the pooled index, progression of radiographic damage and time-integrated functional disability scores may be used in cost-effectiveness ratios.

\section{Efficacy measures; utilities}

In addition to traditional measures of efficacy, utilities by standard gamble and rating scale methods were measured at baseline, and weeks 28 and 56 . A utility is a single comprehensive outcome measure that reflects the value or preference that respondents assign to a particular health state. This value is expressed on a scale ranging from 1 (perfect health) to 0 (death), and takes into account both the positive treatment effects and the negative side-effects. We elicited utilities from patients participating in the trial by means of the Maastricht Utility Measurement Questionnaire, a reliable and validated adaptation in Dutch of the MacMaster Utility Measurement 
Questionnaire [16,17]. It is administered as an interview. Utility measurement using this questionnaire comprises the rating scale and the standard gamble methods. The rating scale measures utilities directly by asking the patients to place health states on a thermometer scale (i.e. vertical visual analogue scale). The standard gamble method derives utilities from the patients' responses to decision situations under risk. A chance board with a probability wheel was used as a visual aid to facilitate the standard gamble questions.

The quality adjusted life year (QALY) is a measure that expresses effects in terms of both quality of life and survival. A calculation of the area under the curve of the baseline and biannual assessments of utility yielded an approximation of QALY gained in each treatment group. We restricted our time window to the first 56 weeks of the protocol in every patient, i.e. we did not extrapolate by multiplying the result with a life expectancy approximation. Direct costs in both treatment groups were related to the gained QALYs to yield cost-utility ratios.

\section{Sensitivity analysis}

Sensitivity analysis tested the robustness of the cost estimates obtained. Volumes, as well as prices, especially those solely based on assumptions, were varied to evaluate the resulting change in the costs. The focus was on the influence of set prices of hospital admissions and help at home, as in this study other volumes were based on empirical data. Each of these two prices were varied by adding and subtracting $25 \%$. The relative price for help by non-professionals was also reduced from the original $80 \%$ to $0 \%$ of professional charges. Monitoring costs were set at a fixed level in the primary analysis. The monitoring schedule is the consensus of the trial rheumatologists achieved in completion of the study. The economic consequences of different monitoring schedules (i.e. a $25 \%$ increase) were considered, as not only the frequency, but also the extensiveness of the monitoring schedule for combined treatment is open to discussion. The monitoring costs for treatment with sulphasalazine alone were derived from a published ACR recommendation [18]. Here, we did not change the frequency of laboratory checks as our protocol agreed with this standard.

\section{Statistical analysis}

To analyse the difference in costs between the combined-treatment and sulphasalazine group, costs per patient-year (of 56 weeks) were calculated and expressed as means per patient per group. As costs are cumulative, the mean is a useful statistic because of its direct relation to the sum. Although the distribution of costs was skewed, the number of patients in our trial permitted parametric (Student's $t$ ) testing for between-group differences in mean aggregate costs (central limit theorem). In the secondary analyses on the differences in volumes, nonparametric MannWhitney tests were preferred. All differences in volume were tested per semester because a priori maximal contrast was expected in the first semester considering the withdrawal of combined treatment after week 28. No adjustments were made for multiple testing. All analyses were performed on an intention-to-treat basis. 


\section{Results}

In one patient (randomized to combined treatment), protocol medication was stopped within one week because his disease was in remission at baseline. Another patient (randomized to sulphasalazine treatment) dropped out in week 2 due to adverse effects (skin rash) and was lost to follow-up for most cost parameters. Data from these two patients were excluded from the analysis. Consequently, the combined-treatment group included 76 patients and the sulphasalazine group 78 patients. Data on costs were complete for all these patients. Both groups were balanced in most important demographic and prognostic variables (Table 4.1).

Table 4.1: Baseline characteristics of the study patients, according to treatment group*

\begin{tabular}{|c|c|c|}
\hline & $\begin{array}{c}\text { Combined } \\
\text { treatment } \\
(n=76)\end{array}$ & $\begin{array}{l}\text { Sulpha- } \\
\text { salazine } \\
(n=78)\end{array}$ \\
\hline Age (yr) & $49 \pm 12$ & $49 \pm 12$ \\
\hline Female & $50(66 \%)$ & $40(51 \%)$ \\
\hline Educational years & $10 \pm 3$ & $10 \pm 3$ \\
\hline Married & $69(91 \%)$ & $71(91 \%)$ \\
\hline Professional occupation $>2$ days/week & $25(33 \%)$ & $26(33 \%)$ \\
\hline Disease duration (months) & $4(1-24)$ & $4(1-23)$ \\
\hline Previous treatment with antimalarials & $16(21 \%)$ & $19(24 \%)$ \\
\hline Hospitalized for RA at baseline & $9(12 \%)$ & $11(14 \%)$ \\
\hline Positive IgM rheumatoid factor & $59(78 \%)$ & $57(72 \%)$ \\
\hline Erosions on hand or foot radiographs ${ }^{\dagger}$ & $55(74 \%)$ & $59(79 \%)$ \\
\hline Number of tender joints $(0-68)$ & $25 \pm 14$ & $24 \pm 14$ \\
\hline Grip strength $(\mathrm{kPa})$ & \pm 15 & $29 \pm 20$ \\
\hline Erythrocyte sedimentation rate $(\mathrm{mm} / \mathrm{h})$ & \pm 34 & \pm 32 \\
\hline Global assessment observer (mm; 0-100) & \pm 24 & $51 \pm 22$ \\
\hline MACTAR functional ability questionnaire & \pm 4 & $24 \pm 4$ \\
\hline Health assessment questionnaire (0-3) & \pm 0.7 & $1.4 \pm 0.7$ \\
\hline
\end{tabular}

* mean \pm S.D., count (\%) or median (minimum-maximum).

$\dagger$ patients with available baseline radiographs; combined treatment $n=74$, sulphasalazine group $n=75$.

The mean total costs per patient in the first 56 weeks of follow-up were $\$ 5519$ for combined treatment and $\$ 6511$ for treatment with sulphasalazine alone $(P=0.37$; Table 4.2). Out-patient care, in-patient care and non-health care each contributed about one-third to the total costs. In the first semester, total costs were almost twice those in the second semester. By definition, combined treatment (protocol drug cost) was more expensive than sulphasalazine: $\$ 326$ vs \$181 per patient. Likewise, the monitoring schedules cost $\$ 839$ per year in the combined-treatment group vs $\$ 559$ in the sulphasalazine group (Table 4.3). The costs of drugs outside the protocol were significantly lower in the combined-treatment group ( $\$ 237$ vs $\$ 329 ; P<0.001)$. This was mainly due to lower use of NSAIDs, analgetics and gastroprotective drugs in the combined-treatment group, especially during the first semester. 
Table 4.2: Direct costs of treatment in 56 weeks of follow-up

\begin{tabular}{|c|c|c|c|c|c|c|c|c|c|c|c|c|c|c|c|}
\hline \multirow[b]{2}{*}{ Direct costs (US\$) } & \multicolumn{5}{|c|}{ 1st semester (week 0-28) } & \multicolumn{5}{|c|}{ 2nd semester (week 29-56) } & \multicolumn{5}{|c|}{ 1st year (week 0-56) } \\
\hline & $\begin{array}{l}\text { Combined } \\
\text { treatment } \\
\text { mean (SEM) }\end{array}$ & $\begin{array}{l}\text { Sulpha- } \\
\text { salazine } \\
\text { mean (SEM) }\end{array}$ & Diff & $\begin{array}{l}\text { fference } \\
95 \% \text { C.I. }\end{array}$ & $P$ & $\begin{array}{l}\text { Combined } \\
\text { treatment } \\
\text { mean (SEM) }\end{array}$ & $\begin{array}{l}\text { Sulpha- } \\
\text { salazine } \\
\text { mean (SEM) }\end{array}$ & Diff & $\begin{array}{l}\text { ference } \\
95 \% \text { C.I. }\end{array}$ & $P$ & $\begin{array}{c}\text { Combined } \\
\text { treatment } \\
\text { mean }\end{array}$ & $\begin{array}{l}\text { d Sulpha } \\
\text { salaz. } \\
\text { mean }\end{array}$ & Differe & $\begin{array}{l}\text { rence } \\
95 \% \text { C.I. }\end{array}$ & $P$ \\
\hline \multicolumn{16}{|l|}{$\begin{array}{l}\text { Health care } \\
\text { Intervention: }\end{array}$} \\
\hline Protocol drug(s) & 190 & 88 & 102 & & * & 137 & 93 & 44 & & * & 326 & 181 & 146 & & * \\
\hline Monitoring & 652 & 409 & 243 & & * & 187 & 150 & 37 & & * & 839 & 559 & 280 & & $*$ \\
\hline Non-protocol drugs & 130 & 186 & -56 & & 0.003 & 108 & 143 & -35 & & 0.06 & 237 & 329 & -92 & & $<0.001$ \\
\hline Other & 364 & 444 & -80 & & 0.08 & 378 & 414 & -36 & & 0.03 & 742 & 858 & -116 & & 0.38 \\
\hline Out-patient care; total & $1336(39)$ & $1127(34)$ & 208 & $113 ; 303<$ & $<0.001$ & $810(25)$ & $800(32)$ & 10 & $-70 ; 91$ & 0.79 & 2,146 & 1927 & 219 & $62 ; 375$ & 0.007 \\
\hline In-patient care & $1002(478)$ & $1868(390)$ & -866 & $-2076 ; 343$ & 0.16 & $532(304)$ & $582(286)$ & -50 & $-874 ; 772$ & 0.90 & 1,534 & 2451 & -917 & $-2573 ; 739$ & 0.27 \\
\hline Non-health care & $1018(205)$ & $1223(178)$ & -205 & $-739 ; 329$ & 0.45 & $821(182)$ & $911(134)$ & -90 & $-534 ; 356$ & 0.69 & 1,840 & 2133 & -293 & $-1232 ; 645$ & 0.54 \\
\hline Total & $3356(600)$ & $4218(457)$ & -863 & $-2343 ; 617$ & 0.25 & $2163(407)$ & $2293(330)=$ & -130 & $-1161 ; 902$ & 0.80 & 5,519 & 6511 & -992 & $-3204 ; 1220$ & 0.37 \\
\hline
\end{tabular}

Combined treatment: $n=76$; Sulphasalazine: $n=78$. Positive values indicate higher costs for the combined-treatment group. SEM; standard error of the mean.

*; Costs fixed per protocol. 
Apart from intervention under study and non-protocol drugs, the costs of ambulant care were slightly lower in the combined-treatment group. Patients in the combined-treatment group consulted their general practitioners more often, but paid less visits to physiotherapists and ergotherapists (Table 4.4). Taken together, the effect of protocol treatment predominated to make combined treatment $\$ 219$ more expensive than sulphasalazine in out-patient costs $(P=0.007)$.

Table 4.3: Costs of possible monitoring schedules during first 56 weeks of treatment (US\$)

For combined treatment (post hoc consensus rheumatologists, see Methods):

$8 \times$ laboratory tests: ESR, hemoglobin, hematocrit, white blood cell count (WBC) including differentiation, platelet count and mean corpuscular volume, ALAT, ASAT, bilirubin, alkaline phosphatase, glucose, creatinine, sodium, potassium, phosphate, calcium, albumin and total protein, urinalysis (albumin and glucose) 292

$1 \mathrm{x}$ hands and feet radiographs ........................................................................................................ 124

$1 \times$ thorax radiographs .................................................................................................................... 36

$1 \mathrm{x}$ bone densitometry lumbar spine and one hip .......................................................................... 207

$7 \mathrm{x}$ consultation rheumatologist .......................................................................................................... 180

Total 839

For sulphasalazine treatment (post hoc consensus rheumatologists):

$7 \mathrm{x}$ times laboratory tests (see above) 256

$1 \mathrm{x}$ hands and feet radiographs 124

$7 \times$ consultation rheumatologist 180

Total 559

For sulphasalazine treatment according to $A C R$ recommendations [18]:

$7 \times$ laboratory tests: hemoglobin, WBC including differentiation and platelet count 53

$1 \mathrm{x}$ hands and feet radiographs

$7 \mathrm{x}$ consultation rheumatologist 180

Total 357

Costs of in-patient care were mean $\$ 917$ lower for combined treatment compared to sulphasalazine $(P=0.27)$. This was mainly due to a significantly lower number of in-hospital days in admitted patients during the first semester: $515 \approx s 190$ days $(P=0.05)$. In the first semester, the number of hospital admissions was 18 in the combined-treatment group vs 24 in the sulphasalazine group, in the second semester, there were 7 admissions in both groups. Admissions were shorter in the combined-treatment group; mean 11.6 rs 20.1 days.

Total direct non-medical costs were a mean $\$ 1840$ for patients in the combined-treatment group and $\$ 2133$ in the sulphasalazine group $(P=0.54)$. Home help accounted for $94 \%$. Not all of these costs were really spent, as not only professional but also voluntary help by spouse, family or friends was appraised. The demand decreased slightly in the second semester. Patients in the combined-treatment group reported less help than patients in the sulphasalazine group, but this difference was not significant. 
The clinical effects have been fully reported elsewhere [5]. Briefly, at week 28 the mean improvement in terms of the pooled index was 1.4 for the combined-treatment group and 0.8 for the sulphasalazine group $(P<0.0001)$. At week 56 , these values were 1.1 vs 0.9 . Comparison of time-integrated indices (with four follow-up measurements and baseline by definition equal to zero) shows mean improvement of 1.1 vs $0.7(P=0.0001)$. Radiographic damage scores of both group were comparable at baseline. At this time, $23 \%$ of the patients in the combined-treatment group and $19 \%$ in the sulphasalazine group showed no damage. After 56 weeks, the median progression in the radiographic damage score was two points in the combined-treatment group $v s$ six in the sulphasalazine group $(P=0.004)$. Baseline and follow-up data on disability of 154 patients were available (76 combined-treatment and 78 sulphasalazine). Baseline scores of both group were comparable. At week 28, the improvement in the disability score was 1.1 for the combined-treatment group $v s \quad 0.6$ for the sulphasalazine group $(P<0.0001)$. At week 56 , improvement compared to baseline was 0.8 vs $0.6(P=0.06)$. Comparison of 56 weeks timeintegrated scores showed mean improvement scores of 0.83 vs $0.50(P=0.0003)$.

Table 4.4: Volumes of out-patient care. Number of visits to health care professionals

\begin{tabular}{|c|c|c|c|c|c|c|}
\hline & \multicolumn{2}{|c|}{ 1st semester (wk 0-28) } & \multicolumn{2}{|c|}{ 2nd semester (wk 29-56) } & \multicolumn{2}{|c|}{ 1st year (wk 0-56) } \\
\hline & $\begin{array}{l}\text { combined } \\
\text { treatment }\end{array}$ & $\begin{array}{l}\text { sulpha- } \\
\text { salazine }\end{array}$ & $\begin{array}{l}\text { combined } \\
\text { treatment }\end{array}$ & $\begin{array}{l}\text { sulpha- } \\
\text { salazine }\end{array}$ & $\begin{array}{l}\text { combined } \\
\text { treatment }\end{array}$ & $\begin{array}{l}\text { sulpha- } \\
\text { salazine }\end{array}$ \\
\hline General practitioners & 104 & 76 & 108 & 97 & 212 & 173 \\
\hline Physiotherapists & 428 & 706 & 318 & 407 & 746 & 1113 \\
\hline Ergotherapists & 31 & 85 & 11 & 48 & 42 & 133 \\
\hline
\end{tabular}

Utility assessments including baseline and two follow-up assessments were available for 67 patients in the combined-treatment group and 75 in the sulphasalazine group. Baseline utility assessment resulted in similar scores for both treatment groups; scores with the rating scale method were 0.55 (S.D. 0.18) and $0.55(0.20)$ for the combined-treatment and sulphasalazine group, respectively; scores with standard gamble were 0.78 (S.D. 0.16 ) and 0.76 (0.19). At week 28, rating scale utility scores increased by 0.24 (S.E.M. 0.02$)$ in the combined-treatment and $0.15(0.02)$ in the sulphasalazine group $(P=0.006)$. Standard gamble utility scores increased by $0.10(0.02)$ in the combined-treatment and $0.06(0.02)$ in the sulphasalazine group $(P=0.05)$. At week 56 , most of the between-group contrast seen after the first semester was lost: mean improvement with rating scale $0.18(0.03)$ in the combined-treatment $v s 0.16(0.02)$ in the sulphasalazine group, and with standard gamble $0.07(0.02)$ vs $0.07(0.02)$. The area-under-the-curve calculation of the utility scores demonstrated a significantly better gain of $0.06 \mathrm{QALY}$ in the combined-treatment group assessed by rating scale $(P=0.01 ; 95 \% \mathrm{Cl}: 0.02-0.10)$. Assessed by standard gamble, the difference was 0.02 gained QALY $(P=0.33$; $95 \%$ CI: $-0.02-0.06)$. 
The efficacy of the combined treatment in clinical outcomes is superior to sulphasalazine alone. The total costs are slightly lower, although not significantly so in the combined group. Accordingly, relative cost-efficacy favours combined treatment; it will be cost-effective when implemented in patients comparable to those included in this COBRA trial. Likewise, significantly better utility scores and equal costs result in better cost-utility ratios in the combined-treatment group.

No extra toxicity due to the extra medication in the combined-treatment group occurred. On the contrary, significantly fewer patients in the combined-treatment group stopped protocol medication due to adverse events or lack of therapy effect; 6 rs 23 in the sulphasalazine group $(P$ $=0.0008$ ). The drop-outs in the combined-treatment group also occurred later. Loss of bone mineral density in spine and hips was modest, and not significantly different in both groups; in the combined-treatment group, bone density in the lumbar spine decreased by a mean $1.3 \%$ in 56 weeks.

In the sensitivity analysis, when the price of hospitalization was reduced by $25 \%$, the mean total cost remained $\$ 849$ lower in the combined-treatment group. The same adjustment in the charge for help at home resulted in a smaller between-group contrast of $\$ 900$. When the price for help at home by non-professionals was set at zero, mean total costs of combined treatment remained $\$ 443$ lower than costs of treatment with sulphasalazine. Finally, the consequences of adjustments to the monitoring schedule were tested (Table 4.3). The adjusted monitoring costs were $\$ 1049(+25 \%)$ and $\$ 357(-36 \%)$ for combined treatment and sulphasalazine, respectively; this still resulted in $\$ 580$ lower total costs for combined treatment. 


\section{Discussion}

In the setting of a randomized trial, this full economic analysis revealed combined treatment with step-down prednisolone, methotrexate and sulphasalazine to be more effective than sulphasalazine alone at equal or lower costs. The combined-treatment group had lower expenses for non-protocol medication and in-patient care, and lower costs outside the health care system that offset the higher costs for protocol medication and monitoring.

The efficacy of the combined treatment in clinical as well as radiological outcomes is superior to sulphasalazine alone. As the total direct costs in the combined-treatment group were equal or lower, relative cost-efficacy favours combined treatment. Utility scores, as a generic measure of therapy benefits, also favoured combined treatment. Concordant with other reported studies, the scores derived with the standard gamble method were at an absolute higher level and least responsive to change. This has been attributed to risk-aversive attitude of patients with a putatively non-fatal or chronic disease like RA [19].

The time frame of this study is long compared to most other studies, but still relatively short for a chronic disease like RA, and definitely too short to evaluate the incidence of late effects. Continuing follow-up of the included patients will provide important answers on costs and outcomes in the long run. Prednisolone might have induced modest (non-significant, mean <1\%) and partially reversible bone loss, but this did not result in symptomatic fractures or complaints in any of the patients.

Like most clinical trials, this trial was primarily designed to study clinical benefits and thus probably underpowered to prove cost benefits. A post hoc power calculation reveals minimum group sizes of 374 necessary for the between-group difference found to reach the level of significance $(P<0.05$, two-sided). The only significant difference in cost found favoured the sulphasalazine group; i.e. $\$ 219$ lower costs for out-patient care. However, this can be largely attributed to the per protocol (fixed) difference in intervention costs.

Sensitivity analysis showed the robustness of the conclusions in the economic analysis. The direct costs of combined treatment are not significantly lower (and must thus be presumed to be similar to the costs of treatment with sulphasalazine alone). On the other hand, calculations with considerable alternations in the assumptions of charges and extensiveness of monitoring schedules consistently showed lower costs for combined treatment. As the primary analysis of the trial showed significantly less drop-out due to therapy failure and adverse events in the combined treatment group, a less frequent and extensive monitoring schedule than that performed during the trial might be appropriate in normal clinical practice. Also, the necessity of initial bone densitometry - in this analysis still regarded as mandatory for combined treatment - is questionable with regard to the observed modest effects on bone. 
The generalizability of our findings is principally restricted to the health care systems in The Netherlands and Belgium. The health care system in these countries is characterized by universal access and equal facilities for all inhabitants, and small distances between patients' homes and clinical centers. From a North American perspective, patients were frequently hospitalized [20]. Notably, at baseline all included patients had active disease, and restrictions to the number or duration of hospitalizations would most likely have worsened the outcomes in the more frequently hospitalized sulphasalazine group [21]. Moreover, the costs of health care found in this study do not seem to be 'out of range' in comparison with figures from an American health care setting $[9,20]$.

In comparison with sulphasalazine, combined treatment is the dominant therapeutic option, due to equal or lower costs and enhanced efficacy. This full economic analysis confirms that combined treatment with step-down prednisolone, methotrexate and sulphasalazine may be a rational choice in early and active RA. 


\section{References}

1. Gordon DA, Hastings DE. Rheumatoid arthritis. Cinical features: early, progressive and late disease. In: Klippel JH, Dieppe PA, eds. Rheumatology: St. Louis: Mosby, 1994:1-14.

2. Wolfe F, Hawley DJ, Cathey MA. Cinical and health status measures over time: prognosis and outcome assessment in rheumatoid arthritis. J Rheumatol 1991; 18:1290-7.

3. McIntosh $\mathrm{E}$. The cost of rheumatoid arthritis. Br J Rheumatol 1996; 3:781-90.

4. Bosi Ferraz M, Maetzel A, Bombardier C Critical appraisal of economic evaluation published in the field of rheumatology and related disciplines. Arthritis Rheum 1997; 40:1587-93.

5. Boers M, Verhoeven AC, Markusse HM, et al. Randomised comparison of combined step-down prednisolone, methotrexate and sulphasalazine with sulphasalazine alone in early rheumatoid arthritis. Lancet 1997; 350:309-18.

6. Arnett FC, Edworthy SM, Bloch DA, et al. The American Rheumatism Association revised criteria for the classification of rheumatoid arthritis. Arthritis Rheum 1988; 31:315-24.

7. Smythe HA, Helewa A, Goldsmith CH. Independent assessor' and 'pooled index' as techniques for measuring treatment effects in rheumatoid arthritis. J Rheumatol 1977; 4:144-52.

8. Centraal Economisch Plan: The Hague: SDU, 1996.

9. Gabriel SE, Crowson CS, Campion ME, O'Fallon WM. Direct medical costs unique to people with arthritis. J Rheumatol 1997; 24:719-25.

10. Rutten FFH, van Ineveld BM, van Ommen R, van Hout BA, Huijsman R. Directe kosten binnen de gezondheidszorg. In: Kostenberekening bij gezondheidszorgonderzoek; richtlijnen voor de praktijk. Utrecht: van Arkel, 1993.

11. Tugwell P, Bombardier C, Buchanan WW, Goldsmith CH, Grace E, Hanna B. The MACTAR patient preference disability questionnaire: an individualized functional priority approach for assessing improvement in physical disability in clinical trials in rheumatoid arthritis. J Rheumatol 1987; 14:446-51.

12. van der Heijde DMFM, van Riel PL, Nuver-Z wart IH, Gribnau FW, van de Putte LBA. Effects of hydroxychloroquine and sulphasalazine on progression of joint damage in rheumatoid arthritis. Lancet 1989; i:1036-8.

13. van Leeuwen MA, van der Heijde DMFM, van Rijswijk MH, et al. Interrelationship of outcome measures and process variables in early rheumatoid arthritis. A comparison of radiologic damage, physical disability, joint counts, and acute phase reactants. J Rheumatol 1994; 21:425-9.

14. Fries JF, Spitz PW, Young DY. The dimensions of health outcome: the Health Assessment Questionnaire, disability and pain scales. J Rheumatol 1982; 9:789-93.

15. Siegert CEH, Vleming LJ, Vanderbroucke JP, Cats A. Measurement of disability in Dutch rheumatoid arthritis patients. Cin Rheumatol 1984; 3:305-9.

16. Bennett K, Torrance GR, Tugwell P: Methodological challenges in the development of utility measure of health-related quality of life in rheumatoid arthritis. Control Cin Trials 1991 (suppl); 12:118-28.

17. Bakker CH, Rutten-van Mölken MPMH, van Doorslaer EKA, Bennet $\mathrm{K}$, van der Linden $\mathrm{S}$. Health related utility assessment by rating scale and standard gamble in patients with ankylosing spondylitis or fibromyalgia. Patient Educ Counsel 1993; 20:145-52.

18. American College of Rheumatology ad hoc committee on clinical guidelines. Guidelines for monitoring drug therapy in rheumatoid arthritis. Arthritis Rheum 1996; 39:723-31.

19. Goossens MEJB, Rutten-van Mölken MPMH, Leidl RMJ, Bos SGPM, Vlaeyen JWS, Teeken-Gruben NJG. Cognitive-educational treatment of fibromyalgia: Randomized clinical trial. II Economic evaluation. J Rheumatol 1996; 23:1246-54.

20. Lanes SF, Lanza LL, Radensky PW, et al. Resource utilization and cost of care for rheumatoid arthritis and osteoarthritis in a managed care setting. The importance of drug and surgery costs. Arthritis Rheum 1997; 40:1475-81.

21. Vliet Vlieland TPM, Zwinderman AH, Vandenbroucke JP, Breedveld FC, Hazes JMW. A randomised clinical trial of in-patient multidisciplinary treatment versus routine outpatient care in active rheumatoid arthritis. Br J Rheumatol 1996; 35:475-82. 


\section{CHAPTER 5}

\section{LIMITED BONE LOSS DUE TO}

CORTICOSTEROIDS; A SYSTEMATIC

REVIEW OF PROSPECTIVE STUDIES

\section{IN RHEUMATOID ARTHRITIS AND \\ OTHER DISEASES}

Arco Verhoeven \& Maarten Boers

Journal of Rheumatology 1997; 24: 1495-503. 


\section{Limited Bone Loss Due to Corticosteroids; a Systematic Review of Prospective Studies in Rheumatoid Arthritis and Other} Diseases

\section{Abstract}

Objective: To clarify the relation between changes in bone density, the treated disease and dose of corticosteroids prescribed.

Methods: MEDLINE database (1966-95) and bibliographic searches selected cohorts of RA patients and non-RA patients, studied by reliable serial bone density measurements.

Results: Two randomised controlled trials in early RA found greater lumbar bone loss after corticosteroid treatment (pooled effect size at 6 months 3.9\%; 95\% CI: 1.9; 6.0\%). The other studies included $66 \mathrm{RA}$ patients on mean $7 \mathrm{mg}$ prednisone/day; 371 'untreated' RA patients; and 216 non-RA patients on mean $20 \mathrm{mg}$ prednisone/day. Lumbar bone mass changed (weighted mean) $0.0 \%(-0.6 ; 0.7 \%)$ per year in steroid-treated RA, $-0.6 \%(-0.9 ;-0.2 \%)$ in 'untreated' RA and $-4.7 \%(-5.2 ;-4.3 \%)$ in non-RA. Femoral neck changed $-3.0 \%(-4.2 ;-1.8 \%),-0.7 \%(-1.0 ;-0.3 \%)$ and $-1.5 \%(-2.5 ;-0.4 \%)$, respectively. In RA, most bone was lost in the first half year, and in early or uncontrolled disease.

Conclusion: In RA patients bone loss is limited, influenced by the interaction of disease characteristics and low-dose corticosteroid therapy. In contrast, non-RA patients on higher doses of corticosteroids may lose clinically relevant amounts of bone (i.e. $>5 \%$ ) within 1 year. 


\section{Introduction}

Osteoporosis is a common finding in patients with rheumatoid arthritis (RA), especially in women over 50. It is unclear how much of this loss is attributable to treatment, specifically corticosteroids, and how much to the disease itself.

RA is a systemic disease characterized by polyarthritis that impairs mobility and physical function, and a catabolic state. The polyarthritis, most frequently situated in the smaller joints of hands and feet, causes juxta-articular bone loss of the affected joints. In addition, there is often considerable generalized loss of bone mass that predisposes to fractures of vertebrae, hips, wrists and ribs. Beside the effects of corticosteroids and possibly other drugs, the disease itself can cause generalized bone loss, evidenced in untreated RA patients. Potential disease-related causes for this bone loss include systemic actions of inflammatory products associated with disease activity, and changes in circulating hormones, in calcium metabolism, and in load bearing of the skeleton. The interplay of all factors is complex: for example, bone loss correlates independently both with markers of disease activity and with disability. On the other hand, corticosteroid treatment may reduce disease activity and increase mobility, which might offset the negative direct drug effect on bone.

Corticosteroids decrease bone mass by impairing intestinal calcium uptake, thus possibly causing secondary hyperparathyroidism; in addition, corticosteroids depress osteoblast activity and may also directly stimulate osteoclast activity. Chronic corticosteroid therapy can result in significant loss of bone mass, especially in skeletal sites with high proportions of trabecular bone, where bone turnover is highest. For example, vertebral wedge and crush fractures are a frequent complication of long-term steroid-treatment in asthmatic patients; another important site at risk is the proximal femur. The fracture risk correlates with the duration and dosage of corticosteroid therapy and is related to low bone density.

Both in RA and in other diseases it remains unclear how large the effects of corticosteroids on bone really are. The extensive literature on this subject comprises mostly cross-sectional and case-control studies, prone to bias by design. Recently the number of prospective studies has increased. In addition, precise and reliable measurement techniques such as dual energy quantitative computer tomography (DQCT), dual photon absorptiometry (DPA) and dual energy $\mathrm{X}$-ray absorptiometry (DXA) have replaced less reliable techniques such as single photon absorptiometry (SPA) and single energy quantitative computer tomography (SQCT). 


\section{Methods}

\section{Identification of studies}

To locate the primary studies of interest, i.e. those with repeated bone density measurements, we performed computer searches in the MEDLINE database (1966 to 31 December 1995). We defined 3 search clusters, termed osteoporosis, corticosteroids and $R A$.

The cluster osteoporosis comprises all citations containing any of the text or thesaurus words 'osteoporosis', 'osteopenia', 'bone density', 'bone mass', 'densitometry', 'absorptiometry' and 'fractures' (all trees and exploded, all subheadings). Similarly the cluster corticosteroids comprises all citations containing any of the text words 'predniso*', 'corticoster"', 'glucocort"' (* indicates a wild card) or thesaurus words 'anti-inflammatory-agents-steroidal' or 'glucocorticoids-synthetic'. Finally the cluster $R A$ comprises the combined text words 'rheumatoid arthritis' and the thesaurus word 'arthritis, rheumatoid' (not exploded). To obtain all studies on corticosteroid induced osteoporosis (RA and non-RA) we intersected the osteoporosis and corticosteroids cluster. To obtain all studies on osteoporosis in RA we intersected the osteoporosis and RA cluster.

\section{Selection of study cohorts}

First screen; appropriate studies

We excluded animal studies, editorials, letters and reviews, retrospective results and crosssectional studies, studies employing single measurements, studies employing bone mineral density/content measurements other than DQCT, DPA or DXA, and studies without data on either lumbar spine or femoral neck. We also excluded studies on diseases, treatments, or situations that affect bone mass, other than RA and corticosteroids. This includes Cushing's disease, hypogonadism, hyper(para)thyroidism, chronic liver disease, insulin dependent diabetes mellitus, renal insufficiency, anorexia, cancer, Addison's disease and malabsorption syndromes including inflammatory bowel diseases, kidney or liver transplantation, and patients under 18 years.

Second screen; appropriate cohorts

Our study analyzes changes in bone mass over time in 3 types of cohorts: RA patients on systemic corticosteroid treatment, RA patients not on corticosteroid treatment, and non-RA patients on corticosteroid treatment. We excluded any cohort that received treatment other than calcium for osteoporosis, e.g. a treatment arm in a controlled trial. On the other hand we would include the control group receiving placebo treatment in such a trial. We excluded studies that did not provide a clear description of amount, kind and duration of corticosteroid treatment and studies that did not explicitly distinguish between patient groups that did or did not have corticosteroid therapy, or between RA and non-RA patients. To be included, studied RA patients had to meet the American College of Rheumatology classification criteria for RA. We screened titles and abstracts from the search and any study that appeared potentially relevant to at least one of us was retrieved. There were no restrictions towards the language of the articles. The same criteria as noted above were used to screen retrieved articles. 
The bibliographies of the selected articles in this selection and six key review articles on corticosteroid induced osteoporosis were scrutinized for additional studies.

\section{Assessment of study quality}

No formal standardized assessment of the quality of the eligible cohorts was done apart from the selection criteria of prospective data acquirement and clear description of the group treatment. Data derived from phacebo-controlled double blind randomised trials (RCTs) on the bone effects of corticosteroid therapy in RA patients were judged best (class a), and considered separately. Next, data from a control group in a randomised controlled trial was perceived to be of higher quality (class b) than data from any other prospectively followed cohort (class c).

\section{Data extraction}

Once the articles were collected and chosen on the basis of the selection criteria, the data extraction was done by both authors separately. Any discrepancies were resolved by mutual agreement. Where a cohort was reported more than once, the most extensive report was used.

\section{Analysis and statistical techniques}

Primary outcome is the mean loss of bone density expressed as percentage of the initial measurement. In the tables, cohorts are ordered according to the underlying disease (that is RA or non-RA), according to treatment with corticosteroids (yes or no) and prior treatment with corticosteroids, according to validity class ( $\mathrm{b}$ or $\mathrm{c}$ ) and according to daily corticosteroid dose.

We retrieved data on a one year follow-up period from text, tables and figures. In 3 studies we used a linearly interpolation from 2-year follow-up data to obtain a one-year estimate. In one instance, we linearly extrapolated from half year data. Confidence intervals were calculated from the retrieved standard errors of the mean. Most studies reported data on bone density (grams per surface unit), some only on grams per volume of a circumscribed area (content).

When no mean percentage of bone loss was reported, we approximated this value by dividing the absolute change in bone mass in a group by its baseline value. When only percent difference in $Z$-scores was reported (i.e. the percentage of percentage difference in standard deviation units from the distribution of bone density in age- and sex-matched normal subjects), we used these as approximate.

Reported corticosteroid doses represent prednisone equivalents; we weighted the mean corticosteroid dose in each of the cohorts by its sample size to obtain the mean dose of the 2 treated cohort groups (RA and non-RA).

We calculated a pooled effect size of the RCTs on the effects of corticosteroids in RA. Similarly, for each of the 3 main groups of cohorts, we calculated mean weighted changes in lumbar spine and femoral neck. To obtain this mean, we weighted each individual change score $\left(x_{i}\right)$ through multiplication with the inverse of its variance; $\left(w_{i}=1 /\right.$ var $\left.x_{i}\right)$, and then summed the results. The weighted mean is this sum divided by the sum of weights $\Sigma\left[w_{i} x_{j}\right] \Sigma w_{i}$, with variance $\sum \mathrm{w}_{\mathrm{i}}$. 
Table 5.1: Bone loss in the lumbar spine and femoral neck in two randomized controlled trials on the effect of corticosteroids in RA.

\begin{tabular}{|c|c|c|c|c|c|c|c|c|c|c|c|c|}
\hline study & & cohort & & & & & & & corticosteroids & bone mass ch & & \\
\hline first author & [ref] & $\begin{array}{l}\mathbf{n}^{\dagger} \text { wo- post- } \\
\text { men menop. }{ }^{t} \\
(\%) \quad(\%)\end{array}$ & $\begin{array}{l}\text { mean disease } \\
\text { age duration } \\
\text { (yrs) (mo) }\end{array}$ & FI & $\underset{1}{\text { DMARD }}$ & $\begin{array}{l}\text { tech- } \\
\text { nique' } \\
\text { (type) }\end{array}$ & $\begin{array}{l}\text { preci- } \\
\text { sion } \\
(\%)\end{array}$ & $\begin{array}{c}\text { Ca- } \\
\text { suppl. }\end{array}$ & $\begin{array}{ccc}\text { prior peak daily } & \text { dose dose } & \text { dose } \\
\text { use } & \text { d } \\
\left(\mathrm{mg} / \mathrm{d}^{t t}\right) & \left(\mathrm{mg} / \mathrm{d}^{t t}\right)\end{array}$ & $\begin{array}{l}6 \text { months } \\
\text { lumbar spine } \\
(\Delta \%) 95 \% \text { c.i. }\end{array}$ & $\begin{array}{l}1 \text { year } \\
\text { lumbar spine } \\
(\Delta \%) 95 \% \text { c.i. }\end{array}$ & $\begin{array}{l}1 \text { year } \\
\text { femoral neck } \\
(\Delta \%) 95 \% \text { c.i. }\end{array}$ \\
\hline
\end{tabular}

\section{$R A$ patients on corticosteroids}

\begin{tabular}{|c|c|c|c|c|c|c|c|c|c|c|c|c|c|c|c|c|c|c|}
\hline Laan & [22] & 20 & 70 & 45 & 53 & 22 & 0.7 & AU & DQCT/C & 6.1 & - & - & 10 & 7.5 & $-8.2-12.7,-3.7$ & n.a. & n.a. & \\
\hline In Schaardenburg & & 27 & 71 & 71 & 69 & 11 & 1.7 & - & DXA /C & 1.9 & + & - & 15 & 8.0 & $-3,3-4.8-18$ & $-38-58-18$ & -4.1 & $-5.7,-2.6$ \\
\hline
\end{tabular}

RA patients not on corticosteroids

\begin{tabular}{|c|c|c|c|c|c|c|c|c|c|c|c|c|c|c|c|c|c|c|c|}
\hline Laan & 19 & 70 & 30 & 56 & 30 & 0.6 & $\mathrm{AU}$ & DQCT/C & 6.1 & - & - & 0 & $\mathbf{0}$ & +1.3 & $-3.1,5.7$ & n.a. & & n.a. & \\
\hline van Schaardenburg [23] & 20 & 43 & 43 & 70 & 10 & 1.6 & CQ & DXA /C & 1.9 & + & - & 0 & $\mathbf{0}$ & -0.1 & $-1.8,1.6$ & -2.0 & $-4.5,0.5$ & -2.6 & $-4.8,-0.4$ \\
\hline \multicolumn{20}{|c|}{ extra loss in RA patients on corticosteroids } \\
\hline Laan & & & & & & & & & & & & & & 9.5 & $3.4,15.6$ & & & & \\
\hline van Schaardenburg & & & & & & & & & & & & & & 3.2 & $1.0,5.4$ & 1.8 & $-1.3,4.9$ & 1.5 & $-1.1,4.1$ \\
\hline pooled effect size & & & & & & & & & & & & & & 3.9 & $1.9,6.0$ & n.a. & & n.a. & \\
\hline
\end{tabular}

* ; percentage change in absolute (Laan) or Z-score (van Schaardenburg) compared to baseline, negative numbers indicate bone loss. n.a.: not available.

$\dagger ; \mathbf{n}$ : number of patients at the end of follow-up.

¥ ; post-menop.: percentage of post-menopausal women in the total cohort.

II ; FI: functional index, Dutch Health Assessment Questionnaire (range 0-3, 3 signifies severe disability).

$\$$; DMARD; concomitant disease modifying anti-rheumatic drug; AU: intramuscular gold salts, CQ: chloroquine.

I ; DQCT: dual energy quantitative computer tomography in a trabecular region of interest; DXA: dual energy x-ray absorptiometry,

/C: bone mineral content (van Schaardenburg measured bone mineral density in the femoral neck).

** ; precision is defined as the coefficient of variation for repeated measurements.

†† ; Ca-suppl.: +: standard supplement of $500 \mathrm{mg}$ calcium per day. -: no calcium supplement.

$\ddagger \ddagger$; in $\mathrm{mg} /$ day prednisone equivalent. 


\section{Results}

The search yielded 2057 citations. A total of 110 studies were retrieved of which 23 were included. The bibliographic search of $6 \mathrm{key}$ reviews and citations in the selected studies and inquiry among colleagues added one relevant study. One potentially relevant abstract in English pointed to a study in Japanese. This study was not included. The final selection comprises 24 studies, 23 in English and one in German [22-45]. Two studies provided two cohorts. Two others provided three: Gough et al distinguished high-dose steroid users, intermediate dose users and non-users, Hall et al followed an extra cohort in addition to 2 cohorts in a trial on hormone replacement therapy.

We present the extracted data in 4 parts; first the data from cohorts in randomised trials that studied the effect of corticosteroid therapy on bone mass in RA patients (Table 5.1); subsequently cohorts with RA patients on corticosteroid therapy (Table 5.2, upper part), cohorts with RA patients not on corticosteroid therapy (Table 5.2, lower part) and finally cohorts with 'non-RA patients' on corticosteroid therapy (Table 5.3).

\section{RCTs on corticosteroid-induced osteoporosis in RA}

Two randomised trials studied the effect of corticosteroid therapy on bone mass in RA. These trials report on 39 and 47 RA patients, studied for one and 2 years respectively (Table 5.1). Laan et al compared the bone content of early RA patients on low-dose corticosteroids or placebo by DQCT. The authors assessed areas of trabecular and cortical bone in the lumbar spine separately; in trabecular bone they found a difference between the groups after 6 months of $9.5 \%$ (95\% confidence interval [95\%CI] 3.4; 15.6\%; $P=0.003$ ): $-8.2 \%$ (loss) in the corticosteroid group, compared to $1.3 \%$ (gain) in the placebo group. Of note was that this loss was largely reversible after tapering the corticosteroids in the second half year $(5.3 \%$ gain; $P=0.03$ in the corticosteroid group versus $1.5 \%$ loss; $P=$ n.s. in the placebo group).

Van Schaardenburg et al studied spine and femoral bone mass in elderly, mostly early RA patients by DXA. In the lumbar spine they found a difference between corticosteroid and 'placebo' group of $3.2 \%$ (95\% CI: $1.0 ; 5.4 \%, P<0.006)$ after 6 months: $-3.3 \%$ (loss) in the corticosteroid group, compared to $-0.1 \%$ in the 'placebo' group (this group received chloroquine as an antirheumatic drug) (Table 5.1). At one year the difference between the groups in this study had decreased to $1.8 \%(P=$ n.s. $):-3.8 \%$ in the corticoid group and $-2.0 \%$ in the placebo group. In the second year of follow-up, neither group lost bone (data not shown).

The pooled effect size of these 2 studies is $3.9 \%$ (95\% CI: $1.9 ; 6.0 \%$ ), corresponding to the extra bone loss in the lumbar spine of RA patients due to treatment with corticosteroids for 6 months. This figure should be interpreted with caution due to heterogeneity in study populations and the differences between the outcome measures (method and unit of measurement). Van Schaardenburg et al also studied the femoral neck. At this site both treatment groups lost bone mass, especially the corticosteroid group; the difference of $1.5 \%$ between the groups was not significant (Table 5.1). In contrast to the lumbar spine, femoral bone loss continued at the same rate in both groups in year 2 . 
Table 5.2: One-year bone loss in the lumbar spine and femoral neck in 17 cohorts of RA patients, treated with corticosteroids (upper part) or not treated with corticosteroids (lower part).

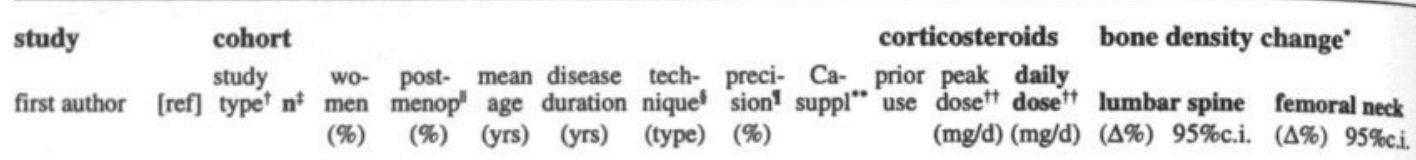

\section{RA patients on corticosteroids}

\begin{tabular}{|c|c|c|c|c|c|c|c|c|c|c|c|c|c|c|c|c|c|}
\hline Messina & [24] & b & 8 & 100 & 0 & 37 & 2 & DXA/C & 1.5 & - & - & 10 & 10 & [+2.2] & n.a. & {$[-7.0]$} & n.a. \\
\hline Gough & [25] & c & 7 & $67^{\text {tt }}$ & 47 & n.a. & $1^{\text {H }}$ & DXA/D & 0.8 & - & - & 5 & $1-5$ & -2.4 & $-6.0,1.2$ & -3.6 & $-8.5,1.3$ \\
\hline Gough & [25] & c & 10 & $67^{\text {tt }}$ & 47 & n.a. & $1^{\# t}$ & DXA/D & 0.8 & - & - & $>5$ & $>5$ & -0.6 & $-3.4,2.4$ & -4.8 & $-8.5,-1.1$ \\
\hline Hall & [26] & b & 16 & 100 & 100 & 56 & 14 & DXA/D & 0.9 & + & + & n.a. & 6 & -0.1 & $-4.5,4.3$ & -0.3 & $-1.2,0.6$ \\
\hline Sambrook '92 & [27] & c & 10 & 100 & 100 & 59 & 17 & DPA/D & 1.8 & n.a. & + & 10 & 6 & -0.5 & $-2.6,1.6$ & -2.5 & $-6.2,1.2$ \\
\hline Sambrook '89 & [28] & c & 15 & 100 & n.a & 55 & 15 & DPA/D & 2.6 & - & + & 10 & 7 & +0.2 & $-0.7,1.1$ & -2.0 & $-3.8,-0.2$ \\
\hline aggregate & & & 66 & & & & & & & & & & 7 & 0.0 & $-0.6,0.7$ & -3.0 & $-4.2,-1.8$ \\
\hline
\end{tabular}

\section{RA patients not on corticosteroids}

\begin{tabular}{|c|c|c|c|c|c|c|c|c|c|c|c|c|c|c|c|}
\hline MacDonald & [29] & b & 13 & 100 & 100 & 55 & 17 & DPA/D & 2.0 & - & 0 & -0.5 & n.a. & n.a. & \\
\hline van den Brink & [30] & b & 18 & 100 & 100 & 63 & 9 & DXA/D & 1.0 & - & 0 & -2.0 & $-8.3,4.3$ & -1.6 & $-11.4,8.2$ \\
\hline Eggelmeijer & [31] & b & 44 & 71 & 25 & 49 & 4 & DXA/D & 1.4 & - & 0 & +0.1 & $-1.8,2.0$ & -2.9 & $-4.5,-1.3$ \\
\hline Sileghem & [32] & b & 10 & 100 & 50 & 54 & 10 & DPA/D & 1.8 & - & 0 & -2.0 & $-3.5,-0.5$ & n.a. & \\
\hline Hall & [26] & b & 67 & 100 & 100 & 56 & 12 & DXA/D & 1.6 & + & 0 & +0.2 & $-1.0,0.6$ & -0.3 & $-1.9,1.1$ \\
\hline Hall & [26] & c & 18 & 100 & 100 & n.a & 12 & $\mathrm{DXA} / \mathrm{D}$ & 1.6 & - & 0 & -0.9 & $-2.0,0.2$ & -0.8 & $-2.3,0.6$ \\
\hline Sambrook ' 85 & [33] & c & 17 & 100 & 71 & 55 & 1 & DPA /C & 1.8 & - & 0 & -1.0 & $-2.2,0.0$ & n.a. & \\
\hline Gough & [25] & c & 85 & $67^{\text {th }}$ & 47 & n.a & $1^{\text {tt }}$ & DXA/D & 0.8 & - & $<1$ & -1.1 & $-1.9,-0.3$ & -1.4 & $-2.5,-0.3$ \\
\hline Sambrook ' 92 & [27] & c & 17 & 100 & 100 & 59 & 11 & DPA/D & 1.8 & n.a. & 0 & +0.2 & $-1.5,1.9$ & -1.1 & $-2.6,0.4$ \\
\hline Sambrook '89 & [28] & c & 15 & 100 & n.a & 55 & 11 & DPA/D & 2.6 & - & 0 & +0.1 & $-0.9,1.1$ & -1.9 & $-3.3,-0.5$ \\
\hline Shenstone & [34] & c & 67 & 60 & 36 & 60 & 2 & DXA/D & n. a. & - & 0 & -1.4 & $-2.9,0.1$ & -0.2 & $-1.3,0.9$ \\
\hline aggregate & & & 371 & & & & & & & & & -0.6 & $-0.9,-0.2$ & -0.7 & $-1.0,-0.3$ \\
\hline
\end{tabular}

\footnotetext{
* ; change in absolute or Z-score (MacDonald) compared to baseline. Negative numbers indicate bone loss. change scores approximated from group means between brackets.

$\dagger$; see methods. ref: reference number, n.a.: not available.

$\ddagger$; number of patients at the end of follow-up (with repeated measurement of lumbar spine at one year follow-up).

$\|$; p-m.w.; percentage of post-menopausal women in the total cohort.

$\$$; DPA: dual photon absorptiometry, DXA: dual energy x-ray absorptiometry, /D: bone mineral density, /C: - content.

I : precision is defined as the coefficient of variation for repeated measurements.

** ; +: standard supplement of 500 or $1000 \mathrm{mg}$ calcium per day, -; no calcium supplement.

$\dagger \dagger$; mg prednisone equivalent per day.

$\ddagger \ddagger$; data refer to the initial group that had baseline assessments ( $\mathrm{n}=154), 2$ women on hormone replacement therapy.
} 


\section{Cohorts with RA patients on corticosteroid therapy}

The RA cohorts on corticosteroid therapy comprise a total of 66 patients (in 6 cohorts), mostly post-menopausal women (Table 5.2, upper part). Patients with both an early and advanced stage of RA were treated with low-dose corticosteroids: mean 7.0, range 1-10 mg prednisone equivalent per day. Changes in bone mass in the lumbar spine ranged between -2.4 (loss) and $2.2 \%$ (gain); weighted mean $0.0 \%$ ( $95 \%$ CI: $-0.6 ; 0.7)$. Bone loss in the femoral neck ranged between -7.0 and $-0.3 \%$; weighted mean $-3.0 \%$ (95\%CI: $-4.2 ;-1.8)$. Gough et al reported that loss of bone occurred preferentially in RA patients with a daily prednisone dose between 1 and 5 $\mathrm{mg}$, as opposed to patients with a dose higher than $5 \mathrm{mg}$ per day.

\section{Cohorts with RA patients not on corticosteroid therapy}

The RA cohorts not on corticosteroid therapy comprise a total of 371 men and women (in 11 cohorts; Table 5.2, lower part). Again, both early and late RA patients were studied. Changes in the lumbar spine ranged between -2.0 and $0.2 \%$; weighted mean $-0.6 \%$ (95\%CI: $-0.9 ;-0.2$ ). Changes in the femoral neck ranged between -2.9 and $-0.2 \%$; weighted mean $-0.7 \%$ (95\%CI: $1.0 ;-0.3)$. Comparison of the weighted means of the 2 cohort groups shows no significant difference in the lumbar spine, but $2.3 \%$ extra femoral bone loss in the steroid cohorts $(P=0.02)$.

\section{Cohorts with non-RA patients on corticosteroid therapy}

The non-RA patient cohorts comprise a total of 216 men and women (in 11 cohorts), treated with corticosteroids for various diseases, including asthma, autoimmune diseases, and heart transplantation (Table 5.3). Approximately half of the patients had previously had corticosteroids or were on maintenance therapy. Peak (median: $50 \mathrm{mg} / \mathrm{d}$ ) and daily dose (mean: $20 \mathrm{mg} / \mathrm{d}$ ) were markedly higher than in the RA cohorts. One-year changes in bone mass in the lumbar spine ranged between -12.5 and $2.4 \%$; weighted mean $-4.7 \%$ (95\%CI: $-5.2 ;-4.3)$. One year changes in the femoral neck ranged between -4.3 and $0.6 \%$; weighted mean $-1.5 \%$ (95\%CI: $-2.5 ;-0.4$ ).

Five studies in non-RA patients presented data on follow-up exceeding one year (data not shown). In giant cell arteritis patients Nordborg et al noted partial reversibility of bone loss on tapering the corticosteroids after one year. Rizzoli et al reported a further increase in lumbar spine density up to $+3.6 \%$ at one and a half year of follow-up in 21 patients. Nelson et al rescanned 8 patients at 2 years of follow-up and reported continued loss of bone density in the lumbar spine. Bijlsma et al reported only 2 year follow-up assessments in 10 patients (interpolated to a one year period in Table 5.3); they found no significant change in lumbar and femoral bone mass. Sambrook et al presented data based on linear regression equations (6 measurements within 2 years in 12 women on chronic steroid therapy); they found stable lumbar bone densities throughout the 2 years in this cohort. In the same study they report on another cohort that started with corticosteroid therapy. This cohort was not included in our review because it did not distinguish between the RA and non-RA patients. However, it is noteworthy that in this cohort with higher baseline values, bone density loss was $-1.7 \%$ per year over 2 years in the lumbar spine, and $-4.6 \%$ in the femoral neck. These losses are larger than in their 'chronic' cohort (Table 5.3) $(P$ $=$ n.s.). 


\section{Discussion}

Our review based on cohorts suggests that short-term ( $\leq 1$ year), low-dose corticosteroid treatment ( $<10 \mathrm{mg}$ prednisone/day) in RA leads to bone loss that is limited in the femoral neck, and negligible in the lumbar spine. Higher dose corticosteroid treatment in non-RA patients led to more marked bone loss in the lumbar spine, but comparable losses in the femoral neck (only 4 series available).

In view of the large body of literature on this subject, we are disappointed with the paucity of prospective data. We were amazed to see that the RA group was twice as large as the non-RA group. Our conclusions must remain tentative in view of the low number of patients in heterogenous settings regarding disease treated, disease activity, previous corticosteroid therapy, corticosteroid dosage, other risk factors (e.g. age and menopausal state), and measurement technique. The relative low number of RA patients on corticosteroid therapy as opposed to RA without steroids and non-RA, is reflected in a wider confidence interval for the estimates of bone loss.

In RA patients Laan et al and van Schaardenburg et al reported significant corticosteroidinduced spinal loss of bone in the only 2 randomised, placebo-controlled trials on the effects of steroid treatment on bone mass. In addition, van Schaardenburg reported significant losses in the femoral neck. The interpretation of the pooled estimate of bone loss is not straightforward possibly even invalid - as the estimates from the 2 studies differ greatly, both in magnitude and in precision. A likely explanation for this discrepancy is method of measurement, another may be age difference. Although both studies reported bone mineral content (instead of bone density), Laan used DQCT and focused on the trabecular bone within the lumbar spine, whereas van Schaardenburg used DXA. DQCT is in itself a less precise method than DXA; in addition, corticosteroid treatment increases marrow fat, spuriously indicating a fall in mineral content as measured by DQCT. Thus the results of van Schaardenburg et al. more likely reflect true bone mass changes.

Our review supports the observation that RA itself can cause discrete bone loss. On aggregate the small losses in spine and femur in the RA patients not on corticosteroids are compatible with normal ageing. However, most cohorts or subgroups with early disease, with active disease, or with more disability showed more marked loss, especially in the femur. In addition, Gough et al showed that patients with early RA, treated with $1-5 \mathrm{mg}$ prednisone daily, lost more bone than patients daily doses above $5 \mathrm{mg}$. This suggests that in early disease, bone loss due to insufficiently controlled RA disease activity can be more important than bone loss due to corticosteroids. Although both Laan $e t$ al and van Schaardenburg et al reported only a short-term beneficial effect of low-dose corticosteroid treatment on RA disease activity, this beneficial effect might still offset the direct detrimental effects of corticosteroids on bone. Compared to the RA cohorts, the nonRA cohorts showed larger spinal bone loss and similar femoral bone loss (4 series) at higher corticosteroid doses. 
Important issues to discuss are the reversibility of treatment effects and the pertinence of short-term prospective follow-up in a situation of longer term treatment. It is heartening that one study in RA patients and two in non-RA noted complete or partial reversibility of bone mass loss after stopping corticosteroids. Regarding long term follow-up, the non-RA patients on corticosteroids showed further loss after intermediate dose corticosteroids, but the RA groups on low-dose corticosteroids ( $<10 \mathrm{mg} / \mathrm{d}$ ) showed stabilization of bone mass in year 2 or 3 . Further, Reid who determined bone loss in RA and non-RA patients by measurement of total body calcium has also concluded that bone loss occurs early in the course of corticosteroid therapy and stabilizes thereafter. Such findings do need confirmation in larger studies. We do not have enough data to discern such a differential effect in our cohorts.

Individual patient factors such as age, sex, menopausal status, disease, disease duration, current and previous corticosteroid dose probably contribute heavily to loss of bone. The design of this review precludes meaningful analysis of risk factors. However, we hope to interest the authors of these and other studies to cooperate with us: we could then pool individual patient data and perform a 'mega-analysis'.

Structured reviews are of necessity out of date by the time they appear in print. For example, Buckley et al. recently published a trial demonstrating that supplementation of calcium together with vitamin D3 prevents spinal bone loss in rheumatoid arthritis patients who were treated with low-dose corticosteroids. One of their control arms would have increased our RA on corticosteroids group by $50 \%$ ( $n=35$, mean daily prednisone dose: $5.6 \mathrm{mg}, 77 \%$ women, $57 \%$ menopausal women, mean age: 54 years, mean disease duration: 8 years). Annual bone losses were $-1.3 \%$ in the lumbar spine and only "tenths of percents" in the femoral neck. Including these findings in our study would have changed our estimates of bone loss only slightly (data not shown).

Loss of bone density in relation to risk of fractures is probably better described by the proportion of the population that suffers from a clinically relevant (that is potentially hazardous) loss of bone density, than by the population's mean decrease. The relation between bone density and fracture risk has only been studied in senile osteoporosis; in this setting bone mineral content may or may not be a better predictor than bone mineral density. In senile osteoporosis, keeping in mind the accuracy of current measurement techniques, one might put the minimum clinically relevant loss of bone density (not bone content) at the femoral neck at $5 \%$, because at this level individuals may have a $50 \%$ increase of their fracture risk. A cohort mean change of $5 \%$ (i.e., half of the individuals within the cohort subject to an additive risk of $50 \%$ or more) was only reached in cohorts of non-RA patients who used more than $10 \mathrm{mg}$ prednisone per day. However, it must be considered that bone quality may be affected differently by RA or corticosteroid treatment than by senile osteoporosis, leading to an increased fracture risk at the same density level. In addition, some RA patients, especially early cases and those with continuously active disease, may lose $5 \%$ bone mass or more regardless of corticosteroid therapy. 
In conclusion, our review of the avalable prospective data suggests that over the course of one year, RA patients may lose discrete amounts of bone mass, especially in early or active dikease; This effect is augmented in the fermur by low-dose corticosteroid therapy. However, assessing the net effect of corticosteroids on bone remains difficult in RA because of beneficial effects on disease activity. In non-RA patients on higher doses of corticosteroids significant losses occur, especially in the spine. In view of the potential reversibility of corticosteroid effects after tapering and stabilization on continued therapy, these findings are probably only clinically relevant in patients where high dose corticosteroid therapy ( $\geq 10 \mathrm{mg} /$ day) is continued for longer periods of time. 


\section{References}

1. Saville PD, Kharmosh O. Osteoporosis of rheumatoid arthritis: influence of age, sex and corticosteroids. Arthritis Rheum 1967; 10:423-30.

2. Peel NF, Spittlehouse AJ, Bax DE, Eastell R. Bone mineral density of the hand in rheumatoid arthritis. Arthritis Rheum 1994; 37:983-91.

3. Bhalla AK, Shenstone B. Bone densitometry measurements in early inflammatory disease. Baillière's Cin Rheumatol 1992; 6:405-14.

4. Laan RFJM, Buijs WCAM, Verbeek ALM, et al. Bone mineral density in patients with recent onset rheumatoid arthritis: influence of disease activity and functional capacity. Ann Rheum Dis 1993; 52:21-6.

5. Lukert BP, Raisz LG. Glucocorticoid-induced osteoporosis: pathogenesis and management. Ann Int Med 1990; 112:352-64.

6. Reid IR: Pathogenesis and treatment of steroid osteoporosis. Cin Endocrinol 1989; 30:83-103.

7. Baylink DJ: Glucocorticoid-induced osteoporosis. NEngl J Med 1983; 309:306-8.

8. Schaadt $\mathrm{O}, \mathrm{Bohr} \mathrm{H}$. Bone mineral in lumbar spine, femoral neck and femoral shaft measured by dual photon absorptiometry with 153-gadolineum in prednisone treatment. Adv Exp Med Biol 1984; 171:201-8.

9. Adinoff $\mathrm{AD}$, Hollister JR. Steroid-induced fractures and bone loss in patients with asthma. NEngl J Med 1983; 309:265-8.

10. Cooper C, Coupland C, Mitchell M. Rheumatoid arthritis, corticosteroid therapy and hip fracture. Ann Rheum Dis 1995; 54:49-52.

11. Cooper C, Barker DJP, Wickham C. Physical activity, muscle strength and calcium intake in fracture of the proximal femur in Britain. Br Med J 1988;297:1443-6.

12. Michel BA, Block DA, Fries JF. Predictors of fractures in early rheumatoid arthritis. J Rheumatol 1991;18:804-8.

13. Laan RFJM, van Riel PLCM, van de Putte LBA. Bone mass in patients with rheumatoid arthritis. Ann Rheum Dis 1992; 51:826-32.

14. Karatanas AH, Kalef-Ezra J, Glaros D. Limitations of quantitative CT in corticosteroid induced osteoporosis. Acta Radiol 1991; 32:339-41.

15. Johnston OC, Slemenda CW, Melton LJ III. Cinical use of bone densitometry. NEngl J Med 1991; 324:1105-9.

16. Arnett FC, Edworthy SM, Bloch DA, et al. The American Rheumatism Association 1987 revised criteria for the classification of rheumatoid arthritis. Arthritis Rheum 1988; 31:315-24.

17. Olbricht T, Benker G. Glucocorticoid-induced osteoporosis: pathogenesis, prevention and treatment, with special regard to the rheumatic diseases. J Internal Med 1993; 234:237-44.

18. Sambrook PN, Jones G. Corticosteroid osteoporosis. Br J Rheumatol 1995; 34:8-12.

19. Dequeker J, Westhovens R. Low-dose corticosteroid associated osteoporosis in rheumatoid arthritis and its prophylaxis and treatment: bones of contention. J Rheumatol 1995; 22:1013-9.

20. DerSimonian R, Laird N. Meta-analysis in clinical trials. Controlled Cin Trials 1986; 7:177-88.

21. Doi Y, Suzuki K, Tanaka M, et al. Measurement of bone mineral density using dual energy X-ray absorptiometry in asthmatic patients receiving prednisolone therapy. Nippon Kyobu Shikkan Gakkai Zasshi 1993; 31:1385-9.

22. Laan RFJM, van Riel PLCM, van de Putte LBA, van Eming LJTO, van ' $t$ Hof MA, Lemmens JAM. Low-dose prednisone induces rapid reversible axial bone loss in patients with rheumatoid arthritis. A randomized, controlled study. Ann Intern Med 1993; 119:963-8.

23. van Schaardenburg D, Valkema R, Dijkmans BAC, et al. Prednisone treatment of elderly-onset RA; disease activity and bone mass in comparison with chloroquine treatment. Arthritis Rheum $1995 ; 38: 334-42$. 
24. Messina OD, Barrein JC, Zanchetta JR, et al. Effect of low doses of deflazacort vs prednisone on bone mineral content in premenopausal theumatoid arthrits. J Rheurnatol 1992; 19:1520-6.

25. Gough AKS, Lilley], Eyre S, Holder RL, Emery P. Generalised bone loss in patients with early rheumatoid arthritis. I ancet $1994 ; 344: 23,7$.

26. Hall GM, Daniels M, Doyle DV, Spector TD, Effect of hormone replacement therapy on bone mass in rheumatoid arthritis patients treated with and without steroids. Arthrits Rherm 199, 37,1499.505.

27. Sambrook P, Birmingham ], Champion GD, et al. Postmenopausal bone loss in theumatoid arthritis: effects of estrogens and androgens. J Rheurnatol 1992; 19:357-61.

28. Sambrook PN, Cohen MI, Eisman JA, Pocock NA, Champion GD, Yates MG. Effects of lowe dose corticosteroids on bone mass in rheumatoid arthritis: a longitudinal study. Ann Rheum Dis 1989; 48:535-8.

29. MacDonald AG, Murphy EA, Capell HA, Bankowsa UZ, Ralston SH Effects of hormone replacement therapy in rheumatoid arthritis: a double blind placebo-controlled study. Ann Rheum Dis $1994 ; 53: 547$.

30. van den Brink HR, Lems WF, van Everdingen AA, Bijlsma JWJ. Adjuvant cestrogen treatment increases bone mineral density in postmenopausal women with rheumatoid arthritis. Ann Rheurn Dis $1993 ; 52: 302-5$.

31. Eggelmeijer F, Papapoulos SE, van Paassen HC, et al. Increased bone mass with pamidronate treatment in rheumatoid arthritis: results of a 3-year randomized, double-blind trial. Arthritis Rheum $1996 ; 39: 396-402$.

32. Sileghem A, Geusens P, Dequeker J. Intranasal calcitonin for the prevention of bone erosion and bone loss in rheumatoid arthritis. Ann Rheum Dis 1992; 51:761-4.

33. Sambrook PN, Ansell BM, Foster S, Gumpel JM, Hesp R, Reeve J. Bone tumover in early meumatoid arthritis. 2. Longitudinal bone density studies. Ann Rheum Dis 1985; 44:580-4.

34. Shenstone BD, Mahmoud A, Woodwand $\mathrm{R}$, et al. Longitudinal bone mineral density changes in carly rheumatoid arthritis. BrJ Rheumatol 1994; 33:341-5.

35. Mulder $\mathrm{H}$, Struys A. Intermittent cyclical etidronate in the prevention of corticosteroid-induced bone loss. Br J Rheumatol 1994; 33:348-50.

36. Olgaard K, Storm T, van Wowern N, et al. Glucocorticoid-induced osteoporosis in the lumbar spine, forearm, and mandible of nephrotic patients: a double-blind study on the high-dose, long-term effects of prednisone versus deflazacort. Calcif Tissue Int 1992; 50:490-7.

37. Nordborg E, Hansson T, Jonson R, Szücs J, Bengtsson B ̊. Bone mineral content of the third lumbar vertebra during 18 month of prednisolone treatment for giant cell arteritis. Cin Rheumatol 1993; 12:455-60.

38. Sambrook PN, Kelly PJ, Keogh AM, et al. Bone loss after heart transplantation: a prospective study. J Heart Lung Transplant 1994; 13:116-20.

39. Luengo M, Picado C, Del Rio L, Guañabens N, Montserrat JM, Setoain J. Treatment of steroidinduced osteopenia with calcitonin in corticosteroid-dependent asthma. A one-year follow-up study. Am Rev Respir Dis 1990; 142:104-7.

40. Nelson HS, Hamilos DL, Corsello PR, Levesque NV, Buchmeier AD, Bucher BL. A double-blind study of troleandomycin and methylprednisolone in asthmatic subjects who require daily corticosteroids. Am Rev Respir Dis 1993; 147:398-404.

41. Bijlsma JWJ, Raymakers JA, Mosch C, et al. Effect of oral calcium and vitamin D on glucocorticoidinduced osteopenia. Cin Exp Rheumatol 1988; 6:113-9.

42. Böhning W, Ringe JD, Welzel D, Bode V. Intranasales Lachscalcitonin zur Prophylaxe des Knochenmineral-verlustes bei Steroid-bedürftigen, chronisch-obstruktiven Atemwegserkrankungen. Arzneim Forsch 1990; 40:1000-3.

43. Worth $\mathrm{H}$, Stammen D, Keck E. Therapy of steroid-induced bone loss in adult asthmatics with calcium, vitamin D, and a diphosphonate. Am J Respir Crit Care Med 1994; 150:394-7. 
44. Rizzoli R, Chevally T, Slosman DO, Bonjour J. Sodium monofluorphosphate increases vertebral bone mineral density in patients with corticosteroid-induced osteoporosis. Osteoporosis Int 1995;5:39-46.

45. Sambrook P, Birmingham J, Kempler S, et al. Corticosteroid effects on proximal femur bone loss. J Bone Miner Res 1990; 5:1211-6.

46. Sambrook P, KellyP, Eisman J. Bone mass and ageing. Baillière's Cin Rheumatol 1993; 7:445-57.

47. Reid DM, Kennedy NS, Smith MA, et al: Bone loss in rheumatoid arthritis and primary generalized osteoarthrosis; effects of corticosteroids, suppressive antirheumatic drugs and calcium supplements. Br J Rheumatol 1986; 25:253-9.

48. Buckley LM, Leib ES, Cartularo KS, Vacek PM, Cooper SM. Calcium and vitamin D3 supplementation prevents bone loss in the spine secondary to low-dose corticosteroids in patients with rheumatoid arthritis; a randomized, double-blind, placebo-controlled trial. Ann Intern Med 1996; 125:961-8.

49. Valkema R, van de Berg R, Camps JAJ, et al. Precision of dual photon absorptiometry measurements: comparison of three different methods of selection of the region of interest. Eur J Nucl Med 1989; 15:183-8.

50. Cummings SR, Black DM, Nevitt MC, et al. Bone density at various sites for prediction of hip fractures. The Study of Osteoporotic Fracture Research Group. Lancet 1993; 341:72-5.

51. Peel NFA, Moore DJ, Barrington NA, Bax DE, Eastell R. Risk of vertebral fracture and relationship to bone mineral density in steroid treated rheumatoid arthritis. Ann Rheum Dis 1995; 54:801-6. 


\section{CHAPTER 6}

\section{RELIABILITY OF SPOT SAMPLES}

FOR ASSESSMENT OF URINARY

EXCRETION OF PYRIDINOLINE

IN PATIENTS WITH RHEUMATOID

\section{ARTHRITIS}

Arco Verhoeven, Maarten Boers, Johan te Koppele, Willemijn van der Laan, Jeroen de Roos, Sjef van der Linden

Clinical and Experimental Rheumatology 2001; 19: 82-4. 


\section{Reliability of Spot Samples for Assessment of Urinary Excretion of Pyridinoline in Patients with Rheumatoid Arthritis}

\section{Abstract}

Objective: To determine how well a spot urine sample of patients with active rheumatoid arthritis (RA) can predict 24-hour urinary pyridinoline and deoxypyridinoline excretion.

Methods: Urine samples of 11 hospitalized RA patients taken on 2 consecutive days at $8 \mathrm{a} . \mathrm{m}$. and 4 p.m. were compared with samples from 24-hour collections (gold standard). High-performance liquid chromatography was used to measure the collagen crosslink concentrations.

Results: Sampling time was the only significant factor (repeated measurement ANOVA). Significant differences were found between morning and 24-hour samples and between morning and afternoon samples, but not between afternoon and 24-hour samples.

Conclusions: Samples collected in the afternoon ( 4 p.m.) give the best approximation of 24-hour urinary pyridinoline excretion in patients with active rheumatoid arthritis. In longitudinal studies the sampling time should be fixed. 


\section{Introduction}

The pyridinium derivatives pyridinoline (PYD = hydroxylysylpyridinoline) and deoxypyridinoline (DPD = lysylpyridinoline) are collagen crosslinks in bone, cartilage and skin. When bone is resorbed, these pyridinium crosslinks are released into the circulation and subsequently excreted in the urine. DPD is present in type I collagen which is the main protein component of bone and dentine. Most excreted DPD comes from bone as this tissue has quick remodelling. Therefore, urinary excretion of DPD is a marker of bone resorption. PYD is more widely distributed and is present in considerable amounts in cartilage-specific collagens: types II, IX and XI. The excretion of PYD and DPD is not influenced by diet or physical exercise. However, a circadian rhythmicity in bone remodelling has been shown for the excretion of pyridinium crosslinks in healthy premenopausal females [1]. It is as yet unclear to what extent a circadian rhythm is present at higher excretion levels of PYD and DPD [2].

Rheumatoid arthritis (RA) is a systemic disease with destruction of joints in hands and feet as a hallmark. This destruction of collagen in bone and cartilage in RA can be accompanied by an increase in PYD and DPD excretion, reflecting disease activity [2]. To date, 24-hour collection to assess excretion is the standard. For practical reasons, spot sampling (or collection of urine during a part of the day) is desirable [3]. The aim of this study was to determine how well a sample of urine of patients with active RA can predict 24-hour pyridinium crosslink excretion.

\section{Methods}

\section{Study design}

Urinary samples were collected from 17 RA patients (ACR 1987 criteria [4]) hospitalized in the Department of Internal Medicine of the Maastricht University Hospital for exacerbation of disease activity. There were no dietary restrictions. Concentrations of PYD, DPD and creatinine $(\mathrm{Cr})$ as well as 24-hour urinary volumes were measured.

Urine collection started at 8 a.m.; a first spot sample was taken at 4 p.m., and a second one the next day at 8 a.m just before completion of 24-hour collection. Ten milliliter aliquots of each sample were stored at $-2^{\circ} \mathrm{C}$. A third aliquot was sampled from the 24-hour collection (after stirring). This procedure was conducted in each patient on 2 consecutive days.

For each patient sex, birth date, RA disease duration (from date of diagnosis), weight, height, erythrocyte sedimentation rate (ESR), medication and serum creatinine were noted. As criterion for appropriate urine collection, we used the ratio of total creatinine excretion on day 1 and day 2; this ratio had to be between 0.8 and 1.25 (not more than 20\% discrepancy between the 2 collections) [4]. 


\section{Pyridinoline and deoxypyridinoline chromatographic analysis}

The total amount of excreted PYD and DPD was measured by high-performance liquid chromatography (HPLC) [5]. Urine samples were hydrolysed in $6 \mathrm{M} \mathrm{HC}$, dried and reconstituted in 50\% acetic acid and injected onto a HPLC system with on-line purification on OC31 cellulose using a Prospekt solid-phase extractor (Separations, The Netherlands). The retained crosslinks were eluted from the $\mathrm{OC} 31$ material and chromatographed on-line on a cation exchange column (Whatman Partisil SCX). Eluted crosslinks were detected by a Jasco fluorometer (Model FP-920, Separations, The Netherlands). The PYD/DPD HPLC Calibrator (Metra, Palo Alto, CA) was used as the standard. Intra- and interassay coefficients of variation were $<3 \%$ and $<5 \%$, respectively, for the PYD and DPD measurements. Urinary excretion rates are expressed as $\mathrm{nmol} / \mathrm{mmol}$ creatinine. Creatinine was measured by the Kodak Ektachem Cinical Chemistry Slide (CREA; Eastman Kodak Company, Rochester, NY, USA).

\section{Statistical analysis}

The PYD/Gr or DPD/Gr ratios in the sample taken from the 24-hour urinary collection ('day sample') were taken as the gold standard. Results are expressed as means with standard deviations. Agreement between each spot sample and the 24-hour collection was expressed as an intra-class correlation (ICC). PYD/Cr and DPD/Cr were analyzed separately. A full repeated measurement ANOVA model quantified variance associated with differences between individuals, between sampling days (day 1 or $2 ; \mathrm{df}=1$ ), between sampling time each day ( 8 a.m., 4 p.m. or 24 -hour collection; $\mathrm{df}=2)$, as well as the interaction between day and sampling time $(\mathrm{df}=2)$ with correction according to Bonferroni-Dunn for repeated testing. Post-hoc contrasts between each spot and 24-hour sample, and between the two samples were calculated.

Table 6.1: Patient characteristics and laboratory values ( $n=11 ; 7$ females and 4 males).

\begin{tabular}{lrrr} 
& & median & $(\min -\max )$ \\
\hline age & $(\mathrm{yrs})$ & 64 & $(42-75)$ \\
disease duration & $(\mathrm{yrs})$ & 4 & $(0.3-34)$ \\
body weight & $(\mathrm{kg})$ & 67 & $(59-98)$ \\
serum creatinine & $(\mathrm{mmol} /)$ & 67 & $(57-152)$ \\
erythrocyte sedimentation rate & $(\mathrm{mm} / 1 \mathrm{st} \mathrm{h})$ & 65 & $(9-95)$ \\
pyridinoline excretion & $(\mathrm{nmol} / 24 \mathrm{~h})$ & 321 & $(140-1015)$ \\
deoxypyridinoline excretion & $(\mathrm{nmol} / 24 \mathrm{~h})$ & 74 & $(32-191)$ \\
creatinine excretion & $(\mathrm{mmol} / 24 \mathrm{~h})$ & 7.7 & $(5.3-11.0)$ \\
pyridinoline/ creatinine excretion & $(\mathrm{nmol} / \mathrm{mmol})$ & 50 & $(23-126)$ \\
desoxypyridinoline/ creatinine excretion & $(\mathrm{nmol} / \mathrm{mmol})$ & 9.5 & $(3.9-18.9)$
\end{tabular}




\section{Results}

Data from 3 patients was excluded because the urinary collection was not accurate (see Patients and Methods). Data from 3 other patients was excluded because PYD/Cr measurements were not available at all 6 points of time. Therefore, data from $11 \mathrm{RA}$ patients was analyzed; 7 women and 4 men. Their mean age was 61 years (range 42-75). They were of normal stature and weight (mean body mass index $26 \mathrm{~kg} / \mathrm{m}^{2}$ ), the mean disease duration was 8 years (median 4) and the ESR was $54 \mathrm{~mm} / \mathrm{hr}$ (median 65). Three patients were on oral corticosteroids (maximum 12.5 $\mathrm{mg}$ prednisone/day). Excretions of PYD and DPD were increased both in absolute terms and when expressed as the ratio crosslinks to creatinine (Table 6.1). The PYD/DPD ratio was similar in the spot and 24-hour samples (mean 5.9, median 5.4).

In the full ANOVA model, sampling time was a significant factor: PYD/Cr (mean \pm SD) was $71 \mathrm{nmol} / \mathrm{mmol} \pm 45$ in samples taken at 8 a.m., $55 \pm 35$ in samples taken at 4 p.m., and $57 \pm 33$ in 24-hour collections $(P=0.01)$. There was no evidence for an effect of measurement day (day 1 or day 2; $P=0.93)$, or for an interaction between day and sampling time $(P=0.72)$. The same applied to $\mathrm{DPD} / \mathrm{Cr}: 14.6 \pm 7.5$ in samples taken at 8 a.m., $10.9 \pm 5.9$ in samples taken at 4 p.m., and 11.5 \pm 5.6 in 24-hour collections $(P=0.02)$. Here again there was no evidence for an effect of the measurement day $(P=0.93)$ or an interaction between the day and sampling time $(P=0.26)$.
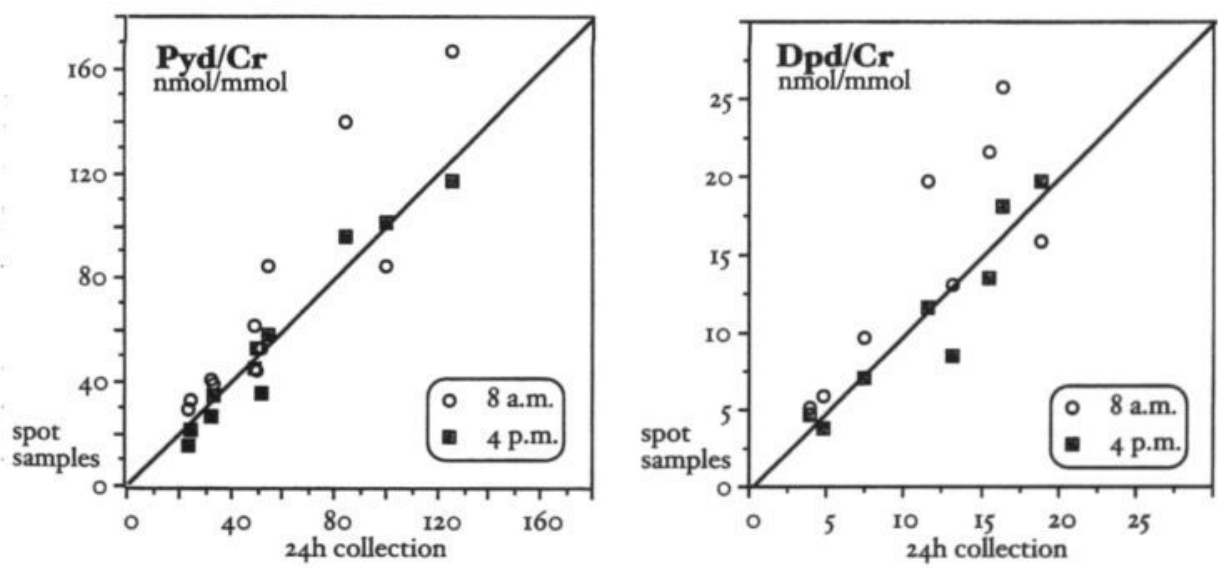

Figure 6.1: Pyridinoline to creatinine ratios (left) and deoxypyridinoline to creatinine ratios (right) in 24-hour urinary collections compared to ratios measured in spot samples taken at 8 a.m. and 4 p.m. All results represent the mean of 2 samples per patient taken on 2 consecutive days. The line of identity is shown for reference. 
Post-hoc contrasts revealed significant differences between morning and 24-hour samples (PYD/Cr, $P=0.02, \mathrm{DPD} / \mathrm{Cr}, P=0.03$ ), and between morning and afternoon samples (PYD/Cr, $P$ $=0.007, \mathrm{DPD} / \mathrm{Cr}, P=0.012$ ), but not between afternoon and 24-hour samples (PYD $/ \mathrm{Gr}, P=0.69$, $\mathrm{DPD} / \mathrm{Cr}, P=0.67$ ). Agreement analysis confirmed these results: $\mathrm{PYD} / \mathrm{Cr}$ in samples taken at 4 p.m. agreed well with values from 24-hour collection samples; the mean difference with the day sample ratio $14 \%$, with an intraclass correlation of 0.96 . For DPD/Cr the mean difference was $16 \%$, intraclass correlation 0.93. Samples taken at 8 a.m. agreed less well: for PYD/Cr, the mean difference with the day sample ratio was $34 \%$ and the intraclass correlation was 0.81 ; for DPD/Cr the mean difference was $37 \%$ and the intraclass correlation was 0.79 . Figure 6.1 shows the 4 p.m. samples close to the line of identity with the day samples (slight underestimation); this contrasts with the overestimation in the 8 a.m. samples.

\section{Discussion}

In rheumatoid arthritis patients with active disease, we observed a circadian rhythm in crosslink excretion at an elevated level. Morning sample values reflect elevated excretion during the night, as has already been observed before in individuals without RA. Therefore morning samples may overestimated 24-hour excretion, whereas afternoon samples are more accurate. Day-to-day variation is small.

Previous studies on circadian rhythms in crosslink excretion were performed in healthy volunteers using 24-hour collection or morning samples taken before breakfast as the gold standard. Consistently, none of these studies found PYD/Gr and DPD/Gr higher than 40 and 8 $\mathrm{nmol} / \mathrm{mmol}$, respectively [6,7]. The hospitalized arthritis patients in this study had modestly raised levels of PYD and DPD excretion level. The specific laboratory methods used may have influenced our results, but the similar PYD/DPD ratios in the spot samples and the 24-hour excretion samples suggest the reliability of the measurement techniques used. Differences in urinary frequency and subsequently the amount of urine in the bladder at moments of sampling may have increased the variance. We applied correction for this by calculating the ratios creatinine excretion but in the experimental settings the timed sampling of portions is preferred (with fasting before morning samples). This sampling schedule is hardly feasible in the routine follow-up of patients, and as our findings were meant to be generalized to the follow-up of ambulant as well as hospitalized patients, we chose a relatively simple sampling method. Despite the small number of patients studied, significant differences were revealed.

In conclusion, the collection of spot samples for the assessment of pyridinoline excretion is best performed in the afternoon ( 4 p.m.), when accurate approximation of average day excretions is the goal. In longitudinal studies, at the very least the sampling time should be fixed. 


\section{References}

1. Fincato G, Bartucci F, Rigoldi M, Abbiati G, Colombo M, Bartolini O, Brandi ML, de Leonardis V. Urinary excretion of pyridinoline and desoxypyridinoline: Circadian rhythm in healthy premenopausal women. J Interdiscpl Cycle Res 1993; 24:72-8.

2. Gough AKS, Peel NFA, Eastell R, Holder RL, Lilley J, Emery P. Excretion of pyridium crosslinks correlates with disease activity and appendicular bone loss in early rheumatoid arthritis. Ann Rheum Dis 1994; 53:14-7.

3. McLaren AM, Isdale $\mathrm{AH}$, Whitting $\mathrm{PH}$, Bird HA, Robins SP. Physiological variations in the urinary excretion of pyridinium crosslinks of collagen. Br J Rheumatol 1993; 32:307-12.

4. Arnett FC, Edworthy SM, Bloch DA, et al. The American Rheumatism Association revised criteria for the classification of rheumatoid arthritis. Arthritis Rheum 1988; 31:315-24.

5. Eyre DR, Koob TJ, van Ness KP. Quantification of hydroxypyridium in cross-links of collagen by high-performance liquid chromatography. Anal Biochem 1984; 137:380-8.

6. Beadsworth LJ, Eyre DR, Dickson IR Changes with age in the urinary excretion of lysyl- and hydroxylysylpyridinoline, two new markers of bone collagen turnover. J Bone Min Res 1990; 5:671-6.

7. Bollen A, Martin MD, Leroux BG, Eyre DR Gircadian variation in urinary excretion of bone collagen cross-links. J Bone Min Res 1995; 10:1885-90. 


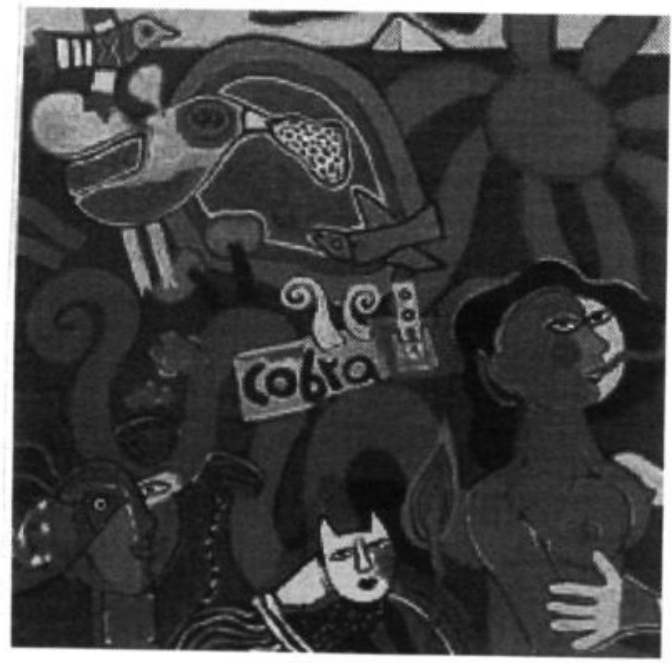




\section{CHAPTER 7}

\section{BONE TURNOVER, JOINT DAMAGE AND BONE MINERAL DENSITY}

IN EARLY RHEUMATOID ARTHRITIS TREATED WITH COMBINATION THERAPY INCLUDING HIGH-DOSE CORTICOSTEROIDS

Arco Verhoeven, Maarten Boers, Johan te Koppele, Willemijn van der Laan, Harry Markusse, Piet Geusens, Sjef van der Linden

Rheumatology 2001; 40: 1231-7. 


\title{
Bone Turnover, Joint Damage and Bone Mineral Density in Early Rheumatoid Arthritis Treated with Combination Therapy Including High-Dose Corticosteroids
}

\begin{abstract}
Objectives: Exploration of bone metabolism changes at different levels of disease activity, both with and without oral corticosteroid therapy, and prediction of changes in joint damage and changes in bone density from the observed changes in markers of bone turnover.
\end{abstract}

Methods: Data analysis from a randomized clinical trial with 155 rheumatoid arthritis (RA) patients; median age 50, early and active disease (diagnosis $<2 \mathrm{yr}$ ); one group treated with a combination of sulphasalazine (SSZ; $2000 \mathrm{mg} /$ day), methotrexate (MTX; $7.5 \mathrm{mg} /$ week) and prednisolone (initially $60 \mathrm{mg} /$ day, tapered in six weekly steps to $7.5 \mathrm{mg} /$ day), the other group with SSZ alone. Prednisolone and MTX were tapered and stopped after week 28 and week 40, respectively, while SSZ was continued. Urine and serum samples were collected at baseline and weeks 16, 28, 40 and 56. Measurements of urinary pyridinoline (PYD) and deoxypyridinoline (DPD) and serum alkaline phosphatase (tAP) and osteocalcin (OC) were performed, as well as standard clinimetry and bone densitometry.

Results: Over time and in both treatment groups, bone formation and bone resorption markers showed a pattern similar to erythrocyte sedimentation index (ESR): A significant decrease compared with baseline and a larger decrease with combined treatment at weeks 16 and 28. PYD excretion, $\mathrm{tAP}, \mathrm{OC}$, and joint damage scores were significant lower in the combined-treatment group. Changes in bone density (of spine and hips) did not significantly differ between treatment groups. Mainly cumulative ESR explained progression of joint damage.

Conclusions: Prednisolone and disease-modifying anti-rheumatic drug therapy in patients with early and active RA are both independently associated with decreased levels of urinary excretion of bone collagen resorption markers: PYD and DPD. Markers of bone formation and resorption closely followed changes in ESR in both treatment groups. Reduced bone resorption together with reduced bone formation - initially at a somewhat faster pace - resulted in less bone turnover and explain the observed (non-significant and partially reversible) extra bone loss in the lumbar spine associated with prednisolone (combined treatment). 


\section{Introduction}

Rheumatoid arthritis (RA) is a systemic disease with inflammation and destruction of joints in hands and feet as a hallmark. As a result of pathological destruction of collagen in bone and cartilage, crosslinks in mature collagen are resorbed more rapidly. This causes a rise in circulating collagen crosslink levels and their urinary excretion. In RA, apart from the crosslink resorption at the site of inflamed joints, there might be increased resorption due to general bone loss associated with disease activity $[1,2]$.

The most important collagen crosslinks of bone and cartilage are the pyridinium derivatives, pyridinoline (PYD or hydroxylysylpyridinoline) and deoxypyridinoline (DPD or lysylpyridinoline). Most DPD is present in type I collagen which is the main protein component of bone and dentine. Most excreted DPD comes from bone as this tissue undergoes relatively rapid remodelling. Therefore, urinary excretion of DPD is considered a more or less specific estimate of resorption of bone by osteoclasts. PYD is more widely distributed and present in considerable amounts in cartilage specific collagen: types II, IX and XI; it may present a marker of destruction in cartilage and other soft tissues [3]. Diet or physical exercise do not influence excretion of PYD and DPD. Because of circadian rhythm in bone remodelling [4,5], excretion in 24-hours collection is widely considered the standard, but samples collected during a fixed day time with concentrations corrected for creatinine ( $\mathrm{Cr}$ ) reflect $24 \mathrm{~h}$ excretion levels as well [6].

Treatment in RA traditionally starts with non-steroidal anti-inflammatory drugs (NSAIDs), and continues if necessary with a sequence of progressively toxic so called disease-modifying antirheumatic drugs (DMARDs) [7]. Some DMARDs and corticosteroids provide a degree of disease control, i.e. they decrease disease activity, maintain physical function and attenuate joint damage visible on radiographs [8-11]. Recent studies suggest that early introduction of DMARDs and corticosteroids may be beneficial for patients with recently diagnosed RA [12,13]. However, the use of corticosteroids is controversial due to side-effects, including osteoporosis. The COBRA study [13] (COmbinatietherapie $\mathrm{Bij}$ Reumatoïde Artritis) aimed at rapid disease control in early RA patients by a step-down bridge combination therapy with agents that have overlapping windows of efficacy onset; the regimen comprised a short period of high-dose oral prednisolone. The other components of the combination were methotrexate (MTX) and sulphasalazine (SSZ) as an anchor drug to remain when the other two drugs were tapered and stopped after 6 months to prevent adverse effects while retaining disease control. The combination therapy regimen demonstrated excellent clinical response, comparatively few adverse effects, and less progression in radiographic joint damage.

This report has two main goals. First, to clarify the changes in bone metabolism at different levels of improvement in disease activity over a 56-week follow-up period, both with and without oral corticosteroid therapy. Second, to investigate the possibility to predict changes in joint damage and bone mineral density (BMD) from the observed changes in markers of bone turnover. 


\section{Patients and Methods}

\section{Patients, clinical efficacy measures and adverse effects}

All measures were done in the setting of the COBRA study [13]. This concerned a 1-yr clinical trial with 155 RA patients (1987 American College of Rheumatology (ACR) criteria [14]) aged 1870 , who were randomly assigned to one of two treatment regimes. All patients had early, active disease (diagnosis $<2 \mathrm{yr}$ ). No prior treatment with second-line anti-rheumatic medication apart from anti-malarials was allowed. One group was treated with a combination of SSZ (2000 $\mathrm{mg} /$ day), MTX (7.5 mg/week) and prednisolone (initially $60 \mathrm{mg} /$ day, tapered in six weekly step to $7.5 \mathrm{mg} /$ day). The other group was treated with SSZ and double placebo. Prednisolone and MTX (or the placebos) were tapered and stopped after weeks 28 and week 40, respectively, while SSZ was continued. The total amount of prednisolone prescribed according to the study protocol was $2345 \mathrm{mg}$, the mean dose over the first 28 weeks of follow-up $12 \mathrm{mg} /$ day. All patients had calcium supplementation $(1 \mathrm{~g} /$ day) prescribed for as long as they used prednisolone, and folic acid (1 $\mathrm{mg} /$ day) as long as they used MTX. Vitamin D supplements were given when vitamin D deficiency was present at baseline.

Cinical improvement was expressed by the preliminary ACR criteria [15] that require a minimum of $20 \%$ improvement in tender and swollen joint counts plus a similar improvement in at least three of five remaining core set measures: patient and physician global assessment, pain, acute phase response, and physical function. We also report the number of core set measures with $20 \%$ improvement, and change in the Disease Activity Score (DAS) [16], a composite outcome measure containing the Ritchie tender joint index (RAI) [17], swollen joint count (48 joints assessed), Westergren's erythrocyte sedimentation rate (ESR), and patient's global assessment (10 cm visual analogue scale $)=0.54 \cdot \sqrt{R} \mathrm{AI}+0.065 \cdot$ swollen joint count $+0.33 \cdot \ln ($ ESR $)+0.07 \cdot$ patient global assessment. Improvement in physical function was reflected by a validated Dutch version of the Health Assessment Questionnaire (HAQ) $[18,19]$ completed by the patients.

Bone-related outcomes were joint damage and BMD. Progression in joint damage was assessed by van der Heijde's [8] modification of Sharp's scoring method; a quantitative score for erosions and joint space narrowing visible on radiographs of hands and feet. Radiographs taken at baseline and weeks 28 and 56 were read in sequence by two trained observers, unaware of treatment allocation. BMD in lumbar spine and femoral neck was measured at baseline and weeks 28 and 56 in centres where dual energy X-ray absorptiometry was available. Changes in BMD $\left(\mathrm{g} / \mathrm{cm}^{2}\right)$ were reported by percentage compared with baseline; for the femoral neck, the mean of both sides was calculated. For further analysis, every patient's propensity to lose bone was categorized as male, pre-menopausal female, post-menopausal female, or post-menopausal female with hormonal replacement therapy.

The study protocol was approved by research and medical ethics committees in all participating hospitals (nine clinical centres in The Netherlands and one in Belgium). All patients gave written informed consent before they entered the study protocol between May 1993 and May 1995. 


\section{Biochemistry}

Urinary spot and serum samples were collected at baseline and weeks 16, 28, 40 and 56, and stored in $10 \mathrm{ml}$ aliquots at $-2^{\circ} \mathrm{C}$. The total amount of excreted PYD and DPD was measured by high-performance liquid chromatography (HPLC). Urine samples were hydrolysed in $6 \mathrm{M} \mathrm{HCl}$, dried and reconstituted in 50\% acetic acid and injected onto a HPLC column with on-line purification on CC31 cellulose using a Prospekt solid-phase extractor (Separations, The Netherlands). The retained crosslinks were eluded from the $\triangle C 31$ material and on-line chromatographed on a cation exchange column (Whatman Partisil SCX). Eluded crosslinks were detected by a Jasco fluorometer (Model FP-920, Separations). The PYD/DPD HPLC Calibrator (Metra, Palo Alto, CA, USA) was used as standard. The intra- and interassay coefficients of variation were $<3$ and $<5 \%$ for PYD/Cr as well as DPD/Cr, respectively. Urinary excretion rates are expressed as $\mathrm{nmol} / \mathrm{mmol} \mathrm{Cr}$. $\mathrm{Cr}$ was measured in the same urine samples by the Kodak Ektachem Cinical Chemistry Slide (CREA; Eastman Kodak Incorporate, Rochester, NY). Bone formation was calculated using total serum alkaline phosphatase (tAP). These were routinely assessed and registered by standard laboratory techniques in each clinical centre at every monitoring visit. Thus, they were available more frequently than every 16 or 12 weeks. Finally, serum osteocalcin (OC) was measured in collected serum samples by a commercial kit (N-MidOsteo; Osteometer, Copenhagen, Denmark) and expressed as $\mathrm{ng} / \mathrm{ml}$. Intra- and interassay coefficients of variation of these measurements were $<5$ and $<8 \%$.

\section{Statistical analysis}

Change in comparison to baseline values was calculated for all variables. In the case of a nonGaussian distribution, change scores were transformed (with natural logarithm). Paired and unpaired Student $t$ tests were used for comparisons of changes with baseline and between groups, respectively. Post-hoc subgroup analysis was performed for hormonal status. The ratio of tAP and natural log-transformed PYD excretion (i.e. $t A P / \ln [\mathrm{PYD} / \mathrm{Cr}]$ ) is presented to reflect changes in the balance between osteoblast and osteoclast activity.

To elucidate further the relationships between PYD excretion and disease activity, we calculated Pearson's moment correlation between changes in PYD/Cr and DPD/Cr at week 28, changes in all ACR core set measures, the number of ACR core set measures with at least $20 \%$ improvement, and change in DAS. From the literature, as well as from primary analyses, acute phase reactants such as ESR emerge as adequate measures to reflect disease activity [15,16]. Timeintegrated values of ESR were calculated, as this representation of cumulative acute phase reaction is known to correlate well with progression in joint damage [20]. Time-integrated PYD/Cr, $\mathrm{DPD} / \mathrm{Cr}, \mathrm{ESR}$ and tAP were correlated with progression in joint damage score, and with changes in $\mathrm{BMD}$ of spine and hips.

To determine if the changes in pyridinoline excretion can be explained solely by glucocorticoid-induced changes in disease activity, a multiple regression analysis was carried out with PYD excretion at week 28 as a dependent variable, and ESR change, treatment group allocation and interaction between group and ESR as independent variables. 
The potential contribution of pyridinoline excretion as a predictor of outcome independent of ESR was evaluated by stepwise multiple regression analyses in which natural log-transformed change in joint damage and changes in bone density served as dependent variables. Their respective baseline values, time-integrated ESR and PYD excretion, and treatment served as predictors (independent variables); $F$ to enter $>4.0$.

Table 7.1: Baseline characteristics median (minimum-maximum) or count (\%) of study patients according to treatment group

\begin{tabular}{|c|c|c|c|c|}
\hline \multirow{2}{*}{$\begin{array}{l}\text { Characteristic } \\
\text { Age (yrs) }\end{array}$} & \multicolumn{2}{|c|}{$\begin{array}{c}\text { Combined } \\
\text { Treatment } \\
(n=76)\end{array}$} & \multicolumn{2}{|c|}{$\begin{array}{l}\text { Sulpha- } \\
\text { salazine } \\
(n=79)\end{array}$} \\
\hline & 51 & $23-70$ & 51 & $24-70$ \\
\hline Female & 50 & $66 \%$ & 41 & $52 \%$ \\
\hline pre-menopausal/ post- / HRT & \multicolumn{2}{|c|}{$25 / 17 / 8$} & \multicolumn{2}{|c|}{$21 / 20 / 1$} \\
\hline Disease duration (months) & 4 & $1-24$ & 4 & $1-23$ \\
\hline Previous treatment with antimalarials & 16 & $21 \%$ & 19 & $24 \%$ \\
\hline Hospitalised for RA at baseline & 9 & $12 \%$ & 11 & $14 \%$ \\
\hline Positive IgM rheumatoid factor & 59 & $78 \%$ & 57 & $72 \%$ \\
\hline HLA-DR4 positive * & 44 & $60 \%$ & 39 & $56 \%$ \\
\hline HLA-DR2 positive * & 16 & $22 \%$ & 12 & $16 \%$ \\
\hline $\operatorname{ESR}(\mathrm{mm} / 1 \mathrm{~h})$ & 51 & $2-136$ & 47 & $2-118$ \\
\hline $\mathrm{PYD} / \mathrm{Cr}(\mathrm{nmol} / \mathrm{mmol})$ & 78 & $6-261$ & 80 & $9-441$ \\
\hline $\mathrm{DPD} / \mathrm{Cr}$ (nmol/mmol) & 21 & $1-61$ & 20 & $1-86$ \\
\hline Alkaline Phosphatase (IU/ L) & 85 & $49-343$ & 85 & $44-394$ \\
\hline Osteocalcin (ng/ml) & 6.1 & $1-25$ & 7.9 & $1-44$ \\
\hline Erosions on hand or foot radiographs ${ }^{\dagger}$ & 55 & $74 \%$ & 59 & $79 \%$ \\
\hline Lumbar spine $\mathrm{BMD}\left(\mathrm{g} / \mathrm{m}^{2}\right)^{\ddagger}$ & 1.13 & $.74-1.46$ & 1.11 & $.66-1.54$ \\
\hline Femoral neck BMD $\left(\mathrm{g} / \mathrm{m}^{2}\right)^{S}$ & .93 & $.66-1.31$ & .92 & $.58-1.25$ \\
\hline
\end{tabular}

HRT; hormonal replacement therapy, ESR; erythrocyte sedimentation rate, PYD; pyridinoline, DPD; deoxypyridinoline, $\mathrm{Cr}$, creatinine.

*; Human leukocyte antigen; homozygotic or heterozygotic DR type, assessed in 143 patients.

†; Baseline radiographs available; combined-treatment group $n=74$, sulphasalazine (SSZ) group $n=75$.

† BMD; bone mineral density; combined-treatment group $n=64, \operatorname{SSZ}$ group $n=62$.

f; combined-treatment group $n=61, \mathrm{SSZ}$ group $n=59$. 


\section{Results}

Cinical effects have been reported elsewhere [13]. Briefly; at week 16, a substantial and significant improvement in almost all measures was observed in both treatment groups. Improvement stabilized or increased towards week 28 , with improvement in the combinedtreatment group being almost double that in the SSZ group. After 28 weeks of follow-up, the mean decrease in ESR was $40 \mathrm{~mm} / \mathrm{h}$ in the combined-treatment group vs $27 \mathrm{~mm} / \mathrm{h}$ in the SSZ group $(P=0.0022$; Figure 7.1$)$. Radiographic damage scores in both groups were similar at baseline (Table 7.1). After 56 weeks, the median progression of the radiographic damage score was two points in the combined-treatment group $v s$ six in the $\operatorname{SSZ}$ group $(P=0.004)$. During the first 28 weeks, BMD in the lumbar spine decreased mean $1.2 \%$ in the combined-treatment group $(n=$ 63) vs $0.0 \%(n=62)$ in the SSZ group $(P=0.06)$. In the femoral neck, the corresponding changes were $-0.3 \%$ vs $-0.7 \%(n=59$ and 57$)$. Significantly fewer patients in the combined-treatment group stopped protocol medication because of adverse events or lack of therapy effect; six $v s 23$ in the SSZ group; $P=0.0008$. Withdrawal occurred later in the combined-treatment than in the SSZ group.

Changes in PYD/Gr and DPD/Gr were calculated in 115 patients; 55 in the combinedtreatment group and 60 in the SSZ group. These changes showed a similar pattern to those observed in the other outcome measures such as ESR. At weeks 16 and 28, PYD excretion decreased significantly compared with baseline values (all $P<0.0001$ ). There was a rapid decrease up to week 16 and a slower decrease up to week 28 , with a significant difference between treatment groups at week $28 ; \mathrm{PYD} / \mathrm{Cr} 45 \%$ vs $18 \%$ reduction in the combined-treatment and SSZ groups $(P=0.02)$; DPD/Cr $33 \%$ vs $15 \%$ reduction $(P=0.11$; Figure 7.1$)$. Also, the PYD/DPD ratio showed a decrease in the combined-treatment group at weeks 16 and 28 (between-treatment differences at weeks 16 and 28: $P=0.04$ and 0.02 ).

Change in tAP agreed with the general pattern of decreased disease activity in reaction to treatment (Figure 7.1). At baseline, mean tAP was slightly elevated in both groups. Change scores on tAP were available for 74 patients in the combined-treatment group and 73 in the SSZ group. Similar to ESR, tAP dropped rapidly in the combined-treatment group (within 4 weeks). At week 16 , there was a significant decrease in both groups $(P<0.001)$ and a significantly larger decrease in the combined-treatment group (difference: $21 \mathrm{IU} / \mathrm{ml}, P<0.001$; $95 \%$ confidence interval (CI) 11$31 ; 31 \%$ vs $11 \%$ reduction). In contrast with the changes observed in PYD/Cr and DPD/Cr, the between-group difference in tAP did not increase between weeks 16 and 28 of follow-up. At weeks 16 and 28, serum OC concentrations in the combined-treatment group $(n=56$; SSZ group $n=62)$ showed a significant decrease (all $P<0.0001)$. Maximum and significant between-group contrast occurred at week $16(P<0.0001$; Figure 7.1). This corresponds with a maximum between-group contrast in $\mathrm{tAP} / \ln [\mathrm{PYD} / \mathrm{Gr}]$ ratio at that time $(P=0.09)$.

Post-menopausal women $(n=39)$ tended to have larger reductions in PYD excretion, as well as in tAP, especially in the combined-treatment group (not significant). This might be due to initially higher values along with greater disease activity in these patients. 

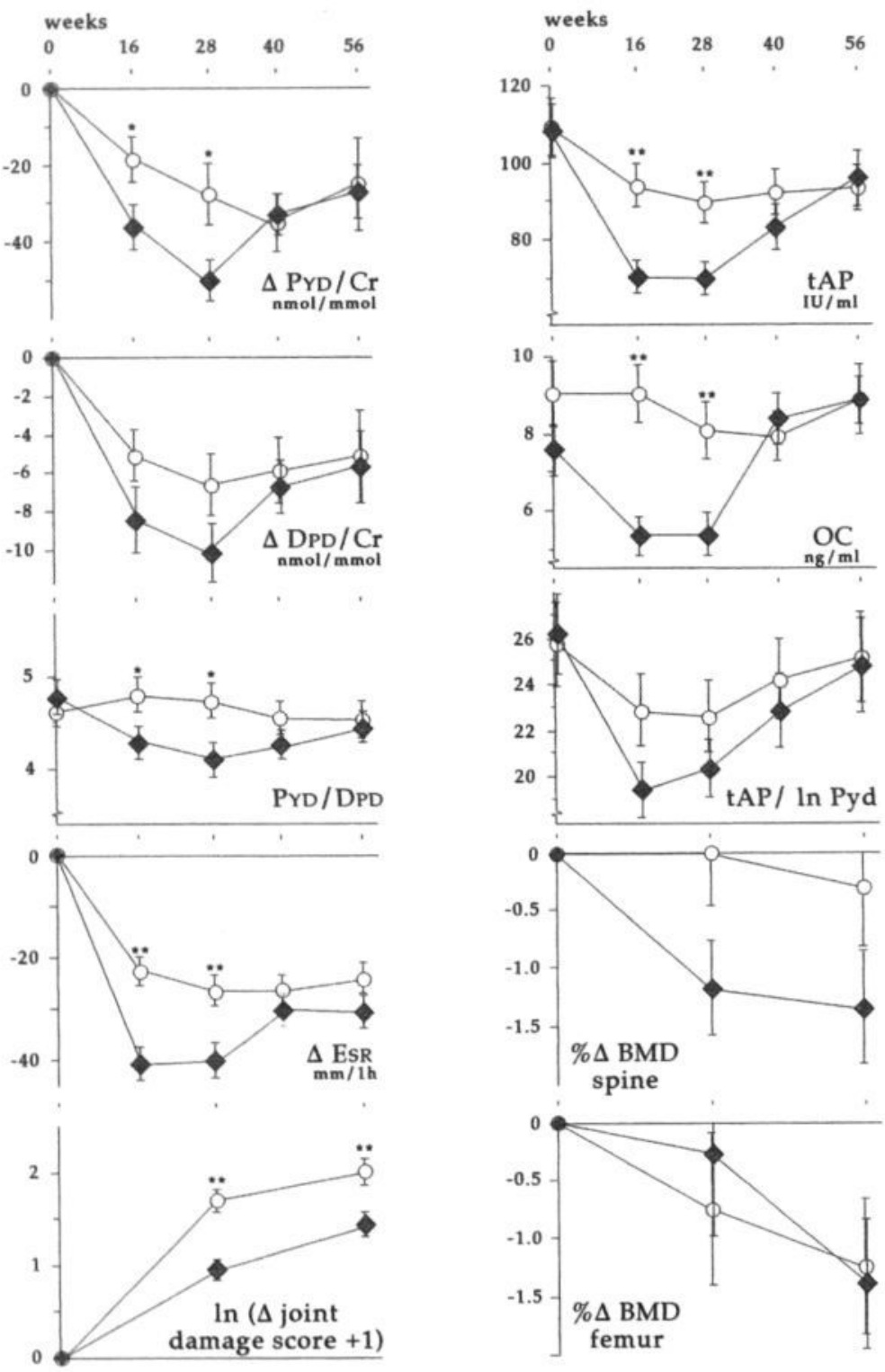

Figure 7.1: Parameters of bone metabolism, acute phase reaction (ESR), joint destruction, and $\mathrm{BMD}$, per treatment group at baseline and follow-up at weeks $16,28,40$, and 56 ; week 40 is after the tapering and withdrawal of prednisolone in the combined-treatment group, week 56 is after the withdrawal of methotrexate. Open circles: SSZ treatment; dark diamonds: combined-treatment. 
Continued legend: Mean \pm S.E.M *: unpaired $t$ test $P<0.05$, **: $P<0.01$

$\triangle \mathrm{PYD} / \mathrm{Cr}$. changes compared with baseline in urinary excretion of pyridinoline/creatinine ratio (nmol/mmol), $\triangle \mathrm{DPD} / \mathrm{Cr}$. changes in urinary excretion of deoxypyridinoline/creatinine ratio, PYD/DPD: ratio, $\triangle E S R$ : change compared with baseline in erythrocytes' sedimentation rate, $\ln (\Delta$ joint damage score +1$)$ : natural logarithm transformed joint damage change score plus one [Sharp/van der Heijde method; erosions and joint space narrowing in hands and feet], tAP: total serum alkaline phosphatase (international units $/ \mathrm{ml})$, OC: serum osteocalcin (ng/ml), tAP/lnPyd: ratio, \% $\%$ BMDspine: percents change compared to baseline in bone mineral density $\left(\mathrm{g} / \mathrm{cm}^{2}\right)$ measured by dual energy $\mathrm{x}$-ray absorptiometry in the lumbar spine, $\% \Delta \mathrm{BMDfemur}$ - in the femoral neck.

Table 7.2 shows correlations of changes in PYD excretion with ACR outcome measures and DAS. Of all core set measures, ESR shows the highest correlation with PYD excretion. Changes in tAP also show significant correlation with changes in PYD excretion. Calculations per treatment group show similar correlations (data not shown).

Table 7.2: Correlations between changes in PYD excretion and changes in other outcome measures at 28 weeks of follow-up

$$
\begin{array}{cc}
\Delta \mathrm{PYD} / \mathrm{Gr} & \Delta \mathrm{DPD} / \mathrm{Gr} \\
(n=115) & (n=108)
\end{array}
$$

$\triangle \mathrm{ESR}$

$\Delta$ global assessment observer

$\Delta$ global assessment patient

$\Delta$ pain

$\Delta$ tender joint count

$\Delta$ swollen joint count

$\triangle \mathrm{HAQ}$

\# ACR 20\%

$\Delta$ Disease Activity Score

$\triangle \mathrm{tAP}$

$\triangle \mathrm{OC}$

.56 ***
$.50 * * \%$
$.25 *$
.20
.22
.15
$.46^{* * *}$
-.36 **
$.33^{* *}$
$.31 *$
.12

.12

\section{$.54^{* * * \%}$}

$.51^{* * *}$

.25

$.25 \%$

.24

.11

$.45^{\text {*** }}$

$-.35^{\text {** }}$

$.31^{*}$

$.33 * \%$

.25

$\Delta$; change score i.e. difference compared to baseline, $\mathrm{HAQ}$; health assessment questionnaire, \# ACR20\%; number of core set measures with $\geq 20 \%$ improvement, OC; osteocalcin, tAP; total serum alkaline phosphate, ${ }^{* * *} P<0.0001$, **; $P<0.001$, *; $P<0.01$.

Change scores for joint damage showed significant correlation with time-integrated pyridinoline excretion: 0.37 and 0.33 for PYD/Cr and DPD/Cr, respectively. Time-integrated ESR and OC yielded larger correlation coefficients. Changes in BMD of the lumbar spine did not yield a significant correlation with PYD excretion. Bone loss in the femoral neck, on the contrary did show a weak and significant association with time-integrated pyridinoline excretion (Table 7.3). 
In multiple regression analyses with change in PYD/Gr and DPD/Gr over 28 weeks as dependent variables, the change in ESR was the only significant attributing variable $(P=0.002$ and 0.011 , respectively, $R^{2}=31 \%$ and $29 \%$ ). Treatment group and interaction between treatment and change in ESR did not significantly increase the proportion of explained variance.

Table 7.3: Spearman's rank correlations of 'bony outcomes' with time-integrated PYD and DPD excretion, ESR, tAP and OC at 28 weeks of follow-up

$\underset{(n=142)}{\Delta \mathrm{X}} \quad \underset{(n=125)}{\Delta \mathrm{BMD}_{\mathrm{LS}}} \quad \underset{(n=116)}{\Delta \mathrm{BMD}_{\mathrm{FN}}}$

$\begin{array}{llrc}\mathrm{PYD} / \mathrm{Cr}_{\mathrm{AUC}} & .37 * * * & -.06 & -.31 * \\ \mathrm{DPD} / \mathrm{Cr}_{\mathrm{AUC}} & .33 * * & -.06 & -.33 * \\ \mathrm{ESR}_{\mathrm{AUC}} & .49 * * * & -.05 & -.15 \\ \mathrm{tAP}_{\mathrm{AUC}} & .11 & .01 & .10 \\ \mathrm{OC}_{\mathrm{AUC}} & .38 * * * & -.11 & -.17\end{array}$

$\Delta$; difference compared to baseline, AUG; area under the curve (time-integrated), $\mathrm{X}$, van der Heijde's modification of Sharp's radiographic joint damage score; $\mathrm{BMD}_{\mathrm{LS}}$; bone mineral density lumbar spine, $\mathrm{BMD}_{\mathrm{FN}}$; bone mineral density femoral neck. *** $P<0.0001$, ** $P<0.001, * P<0.01$.

In a stepwise regression analysis with (log-transformed) progression in joint damage at week 28 as the dependent variable, time-integrated ESR, log-transformed baseline joint damage score, and treatment group entered the model. The proportion of variance explained $\left(R^{2}\right)$ was $53 \%$. Time-integrated PYD excretion did not enter this model. At week 28 of follow-up, changes in $\mathrm{BMD}$ of the lumbar spine and femoral neck proved to be less easy to predict with $R^{2}=5 \%$ and $10 \%$, respectively. For the prediction of spinal bone loss the only variable which was selected was treatment group; for the prediction of changes in femoral bone density, only log-transformed time-integrated $\mathrm{PYD} / \mathrm{Cr}$ was selected. 


\section{Discussion}

Over time and in both treatment groups, PYD excretion showed a pattern similar to the other variables assessed in the COBRA study. Combined treatment with step-down prednisolone, MTX and SSZ caused significantly larger reductions in initially elevated urinary PYD excretion than treatment with SSZ alone. The decreased levels of bone resorption together with decreased bone formation point to a state with less bone turnover. Treatment with SSZ alone also led to a decrease of bone turnover, but this effect was smaller. Markers of bone formation and bone resorption reflect overall disease activity, but the measurable response of osteoblasts (i.e. bone formation) was quicker than that of osteoclasts. A rapid decrease in osteoblast activity - compared to a slower decrease in osteoclast activity - can explain the 'early phase' of bone loss observed in RA patients who start treatment with corticosteroids [21].

Alkaline phosphatase is produced in other cells than osteoblasts and a direct effect of trial medication on this marker cannot be excluded. However, as simultaneously measured transaminase and bilirubin levels, as well as afterwards measured $\gamma$-glutamyl transpeptidase levels, did not show significant changes during prednisolone therapy, we can be rather confident that the changes measured in tAP reflect mainly AP's bone fraction. We prefer tAP instead of OC as a marker of bone formation, as $\mathrm{OC}$ is more properly a marker of bone turnover rather than a specific marker of bone formation [22]. Over time, serum OC levels in both treatment groups also mimicked $\mathrm{tAP} / \ln (\mathrm{PYD} / \mathrm{Cr})$ ratios that served as proxy for bone turnover rates - even with storage conditions that were not optimal for OC.

The level of disease control during the first 28 weeks of follow-up achieved with combined treatment was substantial. Markers of bone resorption (PYD and DPD urinary excretion) reflect this, as well as 'proxy' bone formation marker tAP, and clinical outcome measures. In the correlation analysis, changes in bone resorption markers were strongly related with acute phase reaction and functional ability (i.e. HAQ) and to a lesser extent to markers of bone formation. The observed changes in pyridinoline excretion may be mediated by reduced disease activity alone. An effect of prednisolone on bone turnover at doses as prescribed, separate from altered disease activity, was not demonstrable; treatment group allocation did not significantly improve prediction of changes in PYD excretion once changes in ESR were taken into the regression model.

In the prediction of progression in joint damage in this patient group with early disease, the contribution of observed changes in bone resorption turned out to be modest, especially when markers of acute phase reaction, such as ESR, were available. The relatively high correlation coefficients between cumulative ESR and joint damage score in the SSZ group might be explained by larger variation in therapy effects with less adequate suppression of disease activity through single SSZ therapy. The modified Sharp score comprises joint erosions as well as joint-space narrowing. Bone-specific DPD levels were expected to correlate less with narrowing scores than PYD, because narrowing reflects loss of (joint's) cartilage. This however, could not be demonstrated. 
Decreasing PYD/DPD ratios reflect relatively large resorption of bone as compared with other collagenous tissues. Decreasing levels of PYD, DPD, and PYD/DPD together, as seen in the combined-treatment group, suggest a beneficial effect on predominantly non-bone collagenous tissue; presumably cartilage. In contrast, Sharif et al [23] could not show changes in putatively markers of cartilage loss such as 5D4 keratan sulfate epitope in a RA patient group treated with prednisolone, although there were clear signs of reduced synovitis and bone turnover.

We observed a small extra decrease in spinal BMD in the combined-treatment group $(P=$ $0.06)$. This was most probably a prednisolone effect. After prednisolone was tapered and stopped, the cumulative bone loss stabilized [13]. At this site there was no significant correlation between BMD and cumulative PYD excretion (or disease activity). In contrast, in the femoral neck no difference in BMD loss between the groups emerged, but there was a significant correlation between time-integrated PYD excretion and BMD change. Gough et al earlier described an association between urinary PYD excretion and bone loss at the femoral neck [1]. These BMD changes appear not to be directly mediated by cumulative disease activity, as the correlation with time-integrated ESR is low, and furthermore time-integrated $\mathrm{PYD} / \mathrm{Cr}$ proved to be the only significant predictor for bone loss in the hip. It may be that specifically at the site of the femoral neck corticosteroid-induced bone loss has been balanced by the bone-saving effect of prednisolone through reduction of disease activity [24].

Many pathways of bone metabolism, such as inhibition of matrix metalloproteinases, remain to be clarified. PYD and DPD are valuable markers to show actual bone resorption, especially mechanisms that are not mediated by cytokines or take action at a different level. As an example, those and future bone-specific markers may be necessary to further elucidate the complex relationships between bone metabolism at different sites, RA disease activity and its suppression e.g. by corticosteroids.

In conclusion, the changes in bone metabolism in reaction to treatment with and without corticosteroids, are predominantly mediated by therapy-induced reduction of disease activity. In early RA, the contribution of urinary PYD excretion in the prediction of disease outcome may be modest, especially when markers of acute phase reaction are readily available. Nonetheless, PYD may give us insight into bone metabolism and help us to understand better the actual changes in bone and cartilage caused by RA and its treatment. 


\section{References}

1. Gough AKS, Peel NFA, Eastell R, Holder RL, Lilley J, Emery P. Excretion of pyridinium cross-links correlates with disease activity and appendicular bone loss in early rheumatoid arthritis. Ann Rheum Dis 1994; 53:14-7.

2. Black D, Marabani M, Sturrock RD, Robins SP. Urinary excretion of hydroxy-pyridinium cross-links of collagen in patients with rheumatoid arthritis. Ann Rheum Dis 1989; 48:41-4.

3. Eyre DR, Koob TJ, van Ness KP. Quantification of hydroxypyridinium in cross-links of collagen by high-performance liquid chromatography. Anal Biochem 1984; 137:380-8.

4. McLaren AM, Isdale $\mathrm{AH}$, Whitting $\mathrm{PH}$, Bird HA, Robins SP. Physiological variations in the urinary excretion of pyridinium cross-links of collagen. Br J Rheumatol 1993; 32:307-12.

5. Bollen A, Martin MD, Leroux BG, Eyre DR. Gircadian variation in urinary excretion of bone collagen cross-links. J Bone Min Res 1995; 10:1885-90.

6. Verhoeven AC, Boers M, te Koppele JM, van der Laan WH, de Roos J, van der Linden S. Reliability of spot samples for assessment of urinary excretion of pyridinoline in patients with rheumatoid arthritis. Cin Exp Rheumatol 2001; 19:78-80.

7. Harris ED Jr. Management of rheumatoid arthritis. In: Kelley WN, Harris ED, Ruddy S, Sledge CB, eds. Textbook of Rheumatology, Ch 53. Philadelphia: WB Saunders Company, 1993: 912-23

8. van der Heijde DMFM, van Riel PL, Nuver-Zwart IH, Gribnau FW, van de Putte LBA. Effects of hydroxychloroquine and sulphasalazine on progression of joint damage in theumatoid arthritis. Lancet 1989; i:1036-8.

9. Lopez-Mendez A, Daniel WW, Reading JC, Ward JR, Alarcon GS. Radiographic assessment of disease progression in rheumatoid arthritis patients enrolled in the cooperative systematic disease program randomized clinical trial of methotrexate, auranofin or a combination of the two. Arthritis Rheum 1993; 36:1364-9.

10. Kirwan JR, and The Arthritis and Rheumatism Council Low-dose Glucocorticoid Study Group. The effect of glucocorticoids on joint destruction in rheumatoid arthritis. N Engl J Med 1995; 333:142-6.

11. Edmonds JP, Scott DL, Furst DE, Brooks P, Paulus HE. Antirheumatic drugs: a proposed new classification. Arthritis Rheum 1993; 36:336-9.

12. van der Heide A, Jacobs JWG, Bijlsma JWJ, et al. The effectiveness of early treatment with 'secondline' antirheumatic drugs, a randomized, controlled trial. Ann Int Med 1996; 124:699-707.

13. Boers M, Verhoeven AC, Markusse HM, et al. randomised comparison of combined step-down prednisolone, methotrexate and sulphasalazine with sulphasalazine alone in early rheumatoid arthritis. Lancet 1997; 350:309-18.

14. Arnett FC, Edworthy SM, Bloch DA, et al. The American Rheumatism Association revised criteria for the classification of rheumatoid arthritis. Arthritis Rheum 1988; 31:315-24.

15. Felson DT, Anderson JJ, Boers M, et al. American College of Rheumatology preliminary definition of improvement in rheumatoid arthritis. Arthritis Rheum 1995; 38:727-35.

16. van der Heijde DMFM, van ' $t$ Hof MA, van Riel PLCM, et al. Judging disease activity in clinical practice in rheumatoid arthritis: first step in the development of a disease activity score. Ann Rheum Dis 1990 ; 49:916-20.

17. Ritchie DM, Boyle JA, McInnes JM, et al. Cinical studies with an articular index for the assessment of joint tenderness in patients with rheumatoid arthritis. QJ Med 1968: 147:393-406.

18. Fries JF, Spitz PW, Young DY. The dimensions of health outcome: the Health Assessment Questionnaire, disability and pain scales. J Rheumatol 1982; 9:789-93.

19. Siegert CEH, Vleming LJ, Vanderbroucke JP, Cats A. Measurement of disability in Dutch rheumatoid arthritis patients. Cin Rheumatol 1984; 3:305-9.

20. van Leeuwen MA, van der Heijde DMFM, van Rijswijk MH, et al. Interrelationship of outcome measures and process variables in early rheumatoid arthritis. A comparison of radiological damage, physical disability, joint counts, and acute phase reactants. J Rheumatol 1994; 21:425-9. 
21. Laan RF, van Riel PL, van de Putte LB, van Eming LJ, van ' $t$ Hof MA, Lemmens JA. Low dose prednisone induces rapid reversible axial bone loss in patients with reumatoid arthritis: A randomized, controlled trial. Ann Int Med 1993; 119:963-8.

22. Khosla S, Kleerkoper M. Biochemical markers of bone turnover. In: Favus MJ, ed. Primer on the metabolic bone diseases and disorders of mineral metabolism, Ch 22, 4th ed. Philadelphia: Lippincott, Williams \& Wilkins, 1999: 128-34.

23. Sharif M, Salisbury C, Taylor DJ, Kirwan JR. Changes in biochemical markers of joint tissue metabolism in a randomized controlled trial of glucocorticoid in early rheumatoid arthritis. Arthritis Rheum 1998; 41:1203-9.

24. Verhoeven AC, Boers M. Limited bone loss due to corticosteroids; a systematic review of prospective studies in rheumatoid arthritis and other diseases. J Rheumatol 1997; 24:1495-503. 


\section{CHAPTER 8}

\section{FACTORS PREDICTING RESPONSE}

\section{TO TREATMENT IN RHEUMATOID}

\section{ARTHRITIS: THE IMPORTANCE OF DISEASE DURATION}

Jennifer Anderson, George Wells, Arco Verhoeven, David Felson

Arthritis \& Rheumatism 2000; 43: 22-9. 


\section{Factors Predicting Response to Treatment in Rheumatoid Arthritis: the Importance of Disease Duration}

\section{Abstract}

Objective. To use individual patient data from rheumatoid arthritis (RA) clinical trials to identify factors that affect the response to treatment as defined by the American College of Rheumatology (ACR) criteria for improvement (the "ACR response").

Methods. Primary trial data from 14 diverse, randomized, controlled trials of second-line drugs or devices in RA were analyzed. The trials included 11 methotrexate (MTX) trials (5 placebo controlled and 6 comparative, of which 2 were unpublished), 1 combination trial of cyclosporin plus MTX, 1 induction trial of a combination treatment in early RA (the COBRA trial), and 1 placebo-controlled trial of a new device (Prosorba). Both patient factors and disease activity measures (primarily, items from the ACR core criteria set) were available.

Results. A total of 1,435 patients (549 in placebo-controlled trials, 886 in comparative trials) were studied. In both active treatment and placebo groups, disease duration had a strong effect on the likelihood of patient response (e.g., with any active treatment, the response rate was $53 \%$ for patients with $\leq 1$ year of disease, $43 \%$ for $1-2$ years' disease duration, $44 \%$ for $2-5$ years, $38 \%$ for $5-10$ years, and $35 \%$ for $>10$ years; $P=0.001$ ). Decreasing response with greater disease duration was seen during treatment with most of the individual active drugs, as well as with placebo. Other factors decreasing the rate of response to treatment included any prior use of a disease-modifying anti-rheumatic drug (DMARD), higher disease functional class (according to the Steinbrocker criteria), low disease activity (according to patient's global assessment), and female sex. Each ACR core set variable exhibited a diminished response to treatment in patients with long-standing disease. The difference between active treatment and placebo response rates was not affected by disease duration nor by other factors associated with the ACR response.

Conclusion. RA patients with longer disease duration do not respond as well to treatment compared with patients with early disease, and female sex, prior DMARD use, disease functional class, and disease activity also have effects on the likelihood of patient response to treatment. This has implications for trial interpretation and for the clinical expectations of RA patients. 


\section{Introduction}

It is unknown why some patients with rheumatoid arthritis (RA) respond to treatment better than others. It has been suggested that patients with more severe disease may be less likely to show a response, and that patients who have previously been treated unsuccessfully with second-line drugs also may have more recalcitrant disease [1]. In addition, there are indications that the biologic process of RA changes early in the disease, so that patients may be less responsive to treatment over time [2-5]. The interval in early disease during which response may more readily occur is not clearly defined, so that the relationship between disease duration and response to treatment is of considerable interest. It would also appear that patients with especially active disease are more likely to respond to treatment, particularly in a clinical trial context. Better response in these patients would represent regression to the mean.

An understanding of which types of patients with RA are most likely to respond to treatment has implications for the aggressiveness of treatment of individual patients. In addition, there may be implications for the design of clinical trials, in that the anticipated power of a trial may be a function of the particular mix of patients who participate in the trial. To address these questions, we have made use of patient data from a variety of clinical trials of second-line drugs and devices in RA that have been conducted since the early 1980 s. We selected only trials in which patient response, as defined by the American College of Rheumatology (ACR) criteria for improvement [6] (referred to herein as the "ACR response"), could be determined, and in which individual patient and disease factors were available.

\section{Patients and methods}

We analyzed primary trial data from randomized, controlled trials of second-line drugs or devices in RA. Criteria for selection of the trials included treatment with second-line drugs and the availability of individual patient data on baseline and outcome variables, so that the response rate (according to the ACR 20\% improvement criteria) [6] could be calculated. The ACR improvement criteria require that a patient improve by at least $20 \%$ in each of his or her tender joint count and swollen joint count during a clinical trial, and also improve by at least $20 \%$ in at least 3 of the other 5 core set measures (patient's assessments of pain and physical function, patient's and physician's global assessments, and an acute-phase reactant measure).

We evaluated whether each subject met the ACR criteria for improvement at the end of the trial by an intent-to-treat approach using the last observation carried forward. We did not otherwise make use of any intermediate assessments in assessing whether a patient satisfied the ACR response criteria. In the 3 most recent trials, the Health Assessment Questionnaire (HAQ) [7] disability score was the physical function measure used in the calculation of improvement, while in the earlier trials, grip strength was available as the physical function measure for this calculation. We have previously shown a moderate correlation between change in grip strength and change in self-reported functional status [6]. 
We used logistic regression to analyze the factors affecting the likelihood of patient response, with initial tests performed on each of the candidate factors separately. These factors fell into 2 groups: patient factors (age, sex, disease duration, Steinbrocker functional class [8], any prior use of second-line drugs, and any prednisone use during the trial) and disease activity factors (each of the ACR core set items evaluated at baseline). In all logistic regression analyses, we controlled for treatment, with an indicator variable for each non-placebo treatment. We did not include the trial, or "study," as an effect in these analyses because some active treatments were studied in only 1 trial. It was therefore not possible to control for both study and treatment. We retained, for subsequent multivariate analyses, any patient or disease activity factor with a $P$ value less than 0.15 in its statistical association with the ACR response.

Because of missing data on several items in some trials, we constructed 2 data sets for the multivariate analyses. These were an "all variable" data set, consisting of the trials in which all retained candidate variables were available, and an "all trial" data set, from which some of the retained variables were missing and therefore not analyzable. In the multivariate analyses, we assessed the simultaneous associations of candidate factors with response. Data on the strength of the association identified in the multivariate logistic regressions are expressed as an adjusted odds ratio $\left(\mathrm{OR}_{\mathrm{ed}}\right)$ that represents the odds ratio per unit increase in a variable when other variables are also in the model.

For factors found to have an association with response to treatment, we looked for possible effects on the statistical power of trials. We used logistic regression to test the interaction of active treatment versus placebo with the factor to determine whether the difference in response between active treatment and placebo varied with factor level. In further logistic regressions, we also examined the association between the response ( $\geq 20 \%$ improvement) in individual ACR core set items and these same factors. 


\section{Results}

We analyzed data from 14 RA trials. These included 11 methotrexate (MTX) trials (5 placebo controlled and 6 comparative, of which 2 were unpublished) that were conducted in the $1980 \mathrm{~s}$ and assembled into an archive [9-18] and unpublished observations (from Schmid FR and from Wilke WS). Three additional trials from the 1990 s were also studied: 1 combination trial of cyclosporine plus MTX [19], 1 induction trial of a combination treatment in early RA [20] (referred to as the COBRA trial), and 1 placebo-controlled trial of a new device (Prosorba) [21]. Table 8.1 lists the 14 trials with their publication dates and treatments, as well as the sizes and ACR response rates for each treatment group. The treatments included MTX, auranofin (AUR), the combination of MTX and AUR, sodium aurothiomalate (injectable gold), azathioprine (AZA), cyclosporin, sulphasalazine (SSZ), the combination of MTX, SSZ, and prednisolone, and Prosorba.

We studied 1,435 patients (549 participating in the placebo-controlled trials, 886 in the comparative trials). Overall response rates were higher in the comparative trials (ranging from $38 \%$ to $53 \%$ ) than in the placebo-controlled trials (5\% to $37 \%$ ).

Table 8.2 shows the mean values and range of means for the patient factors and disease activity measures in the 14 trials. In some respects, the trials were similar, in that each had a majority of female patients and the average age of the patients ranged from 50 years to 60 years. The trials varied considerably, however, in average disease duration, which ranged from 0.5 years in the COBRA trial [20] to 15.5 years in the Prosorba trial [21] to 17.7 years in 1 of the small trials of MTX versus placebo (Schmidt FR: unpublished observations). The trials also varied in the distribution of patients according to Steinbrocker functional class (12-47\% of patients in class III per trial), the extent of prior use of disease-modifying antirheumatic drugs (DMARDs) (10-100\% of patients per trial), and the baseline disease activity (for example, the average number of swollen joints ranged from 15 to 30 across the 14 trials).

The "all variable" data set did not include 4 comparative trials because 1 or more of the variables of prior DMARD use, physician's global assessment, or Steinbrocker functional class was either not assessed or not recorded in these 4 trials. The largest of these trials was the COBRA trial, in which only the Steinbrocker functional class was not available for our analyses.

As shown in Table 8.3 , the univariate analyses identified 4 patient factors associated with a reduced likelihood of treatment response: female sex, longer disease duration, higher Steinbrocker functional class, and prior DMARD use. The univariate effects persisted in the multivariate analyses. In multivariate analyses using the "all variable" data set, the odds of response to treatment for women were 0.72 times those for men. Similarly, the odds of response were only 0.60 for patients in Steinbrocker functional class III versus those in functional class II, and prior DMARD use was associated with a similar reduction in the odds of response, to 0.62 . The disease duration effect on odds of response was 0.74 when expressed per 15-year increase in disease duration ( 0.98 per extra year of disease duration). Despite the possibility of strong correlations between these patient factors, particularly between prior DMARD use and disease duration, each maintained an independent effect in the multivariate analysis. 
Table 8.1: Trials included in study of factors affecting response to second-line drug treatment in rheumatoid arthritis*

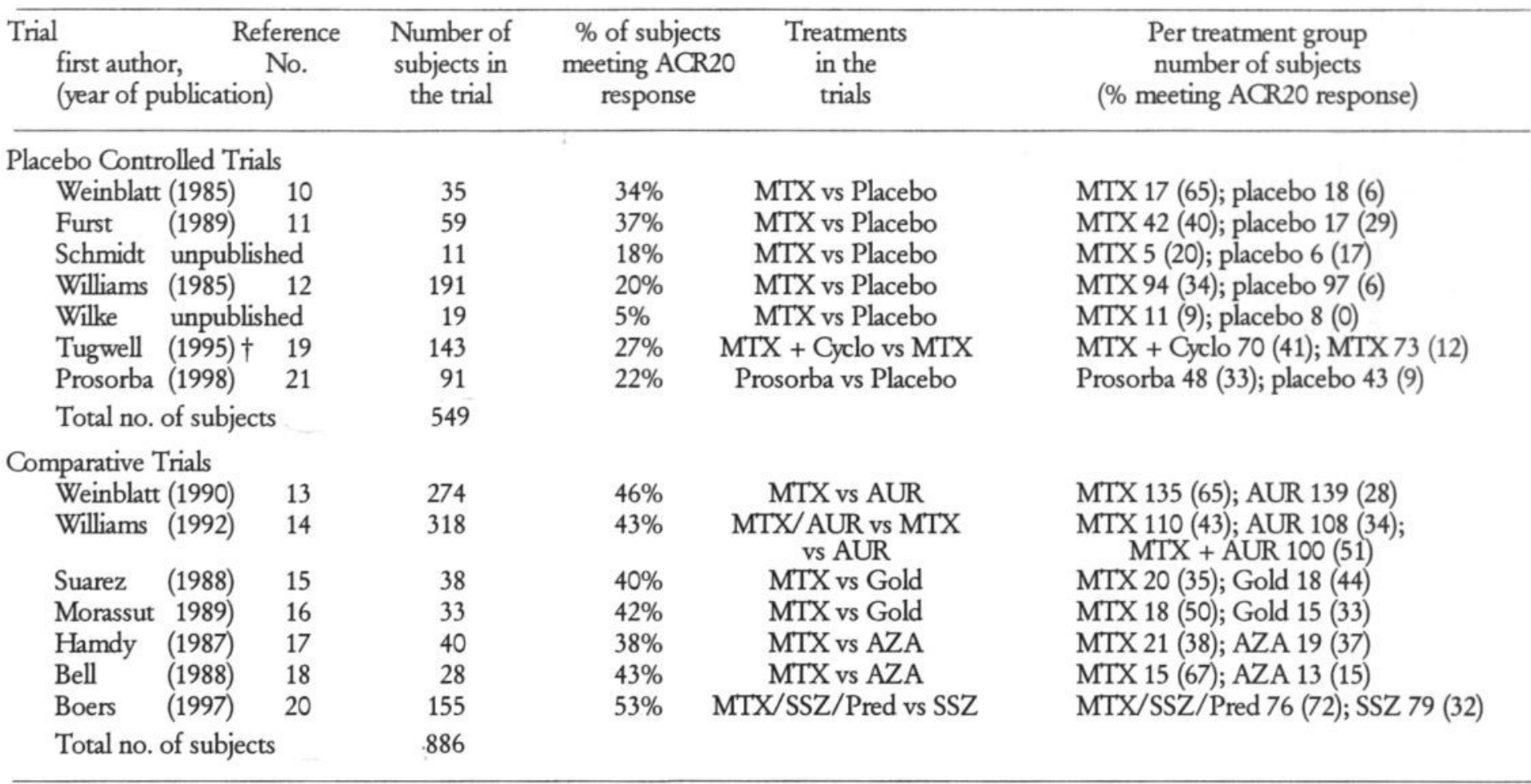

* ACR20 = American College of Rheumatology 20\% improvement criteria [6]; MTX=methotrexate; Cyclo =cyclosporin; $\mathrm{AUR}=$ auronofin; Gold = sodium aurothiomalate; $\mathrm{AZA}=$ azathioprine; $\mathrm{SSZ}=$ sulphasalazine; Pred $=$ Prednisolone.

$\dagger$ In this trial, all patients were being treated with MTX before the trial began, so it was included as a placebo-controlled trial of cyclosporin. 
Among the disease activity measures associated with response were the patient's assessment of pain and the swollen joint count, as well as the patient's and the physician's global assessments. Each of these factors increased the likelihood of response, while the opposite trend occurred for physical function as measured by the HAQ score. In the univariate analyses, the effect of the HAQ score appeared substantial, at 0.77 per unit on the 0-3 scale used for the HAQ. The test of significance had low power, however, with $P=0.15$, because HAQ assessments were available in only 3 of the trials. In the multivariate analysis of the "all variable" data set, the patient's global assessment was the sole significant disease activity variable affecting the likelihood of response.

Table 8.2: Patient factors and disease activity measures across the 14 trials

Overall mean Range of means $\quad \begin{gathered}\text { No. of trials } \\ \text { missing }\end{gathered}$

\section{Patient factors}

\section{Age, years}

Female, \%

Disease duration, years

Steinbocker class III, \%

Prior use of DMARDs, \%

Prednisone use, \%

Disease activity measures

Pain assessment (0-100 scale)

Swollen joint count (of 66 joints)

Tender joint count (of 68 joints)

ESR, $\mathrm{mm} /$ hour

Grip strength, $\mathrm{mm} \mathrm{Hg}$

HAQ score (0-3)

Physician's global assessment ( $0-100$ scale) 56

$\begin{array}{ccc}53 & 50-60 & 0 \\ 69 & 55-91 & 0 \\ 7.8 & 0.5-17.7 & 0 \\ 20 & 12-47 & 4 \\ 45 & 10-100 & 1 \\ 31 & 3-82 & 1\end{array}$

53

22.5

29.7

44

94

1.5

56

58
43- 76

14.8- 30.3

19.5- 37.4

34- 67

52- 140

1.4- 1.9

49. 75

47. 80

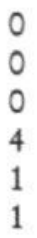

0

4

1

DMARDs: disease-modifying anti-rheumatic drugs; ESR: erythrocyte sedimentation rate; HAQ: Health Assessment Questionnaire.

In the final multivariate analysis, summarised in Table 8.3, among the variables in the "all trial" data set, the results for the variables in common were similar to those for the variables in the "all variable" data set, except that the effect of sex was less strong $\left(\mathrm{OR}_{\mathrm{adj}} 0.81\right.$ versus 0.72$)$ and the disease duration effect was stronger $\left(\mathrm{OR}_{\mathrm{dj}} 0.97\right.$ per year, which translates into 0.63 per 15 years, versus an $\mathrm{OR}_{\mathrm{dj}}$ of 0.98 per year, corresponding to 0.74 per 15 years). 
Table 8.3: Association of patient and disease activity factors with the likelihood of patient improvement *

Multivariate models

\begin{tabular}{|c|c|c|c|c|c|}
\hline \multicolumn{2}{|c|}{ Univariate analyses } & \multicolumn{2}{|c|}{$\begin{array}{c}\text { "All variable" data set } \\
\text { of } 10 \text { trials } \\
(\mathrm{n}=985)\end{array}$} & \multicolumn{2}{|c|}{$\begin{array}{l}\text { "All trial" data set } \\
\text { of } 14 \text { trials } \\
(\mathrm{n}=1,404)\end{array}$} \\
\hline $\mathrm{OR}_{\mathrm{adj}}$ & $P$ & $\mathrm{OR}_{\mathrm{adj}}$ & $P$ & $\mathrm{OR}_{\text {adj }}$ & $P$ \\
\hline
\end{tabular}

\section{Patient factors}

Age (per year)

$\operatorname{Sex}(\mathrm{F})$

Disease duration (per year)

Steinbrocker functional class (per 1)

Any prior DMARDs (yes)

Any prednisone during trial (yes)

$\begin{array}{ll}.99 & .341 \\ .79 & .053 \\ .97 & .001 \\ .66 & .007 \\ .59 & .001 \\ .98 & .871\end{array}$

Baseline disease activity measures

ESR (per mm/hour)

Pain assessment ( $0-10$ scale) (per unit)

$1.00 \quad .512$

Tender joint count (0-68) (per unit)

$1.05 \quad .085$

Swollen joint count (0-66) (per unit)

$1.00 \quad .813$

Grip strength (per $\mathrm{mm} \mathrm{Hg}$ )

$1.01 \quad .064$

HAQ (0-3 scale) (per unit)

$1.00 \quad .419$

Patient's global assessment ( $0-10$ scale) (per unit)

$0.77 \quad .145$

Physician's global assessment ( $0-10$ scale) (per unit)

$1.10 \quad .001$

$1.08 \quad .030$

$\begin{array}{ll}.72 & .040 \\ .98 & .028 \\ .60 & .004 \\ .62 & .008\end{array}$

$.81 \quad .095$

$.97 \quad .001$

$\quad .60 \quad .004$

Alla

All analyses adjusted for treatment. $\mathrm{OR}_{\mathrm{adj}}=$ adjusted odds ratio (see Table 8.2 for other definitions). 
The strong effect of disease duration on response to treatment for most of the different treatments can be seen in Figure 8.1. Among the patients receiving active treatment, there was a response rate of $53 \%$ for those with a disease duration under 1 year, $43 \%$ for a disease duration between 1 year and 2 years, $44 \%$ for 2-5 years' disease duration, $38 \%$ for $5-10$ years' disease duration, and $35 \%$ for patients with a disease duration exceeding 10 years. The pattern of response was similar for the different DMARD treatments, particularly for the treatments in the COBRA trial, in which all but $10 \%$ of participants had $<1$ year of disease. Because there were only 9 subjects treated with Prosorba who had a disease duration under 5 years, the response rate estimate of $22 \%$ ( 2 of 9 subjects) for that range was unstable, but the response was steady at $33 \%$ and $35 \%$, respectively, in the longer ranges of disease duration.

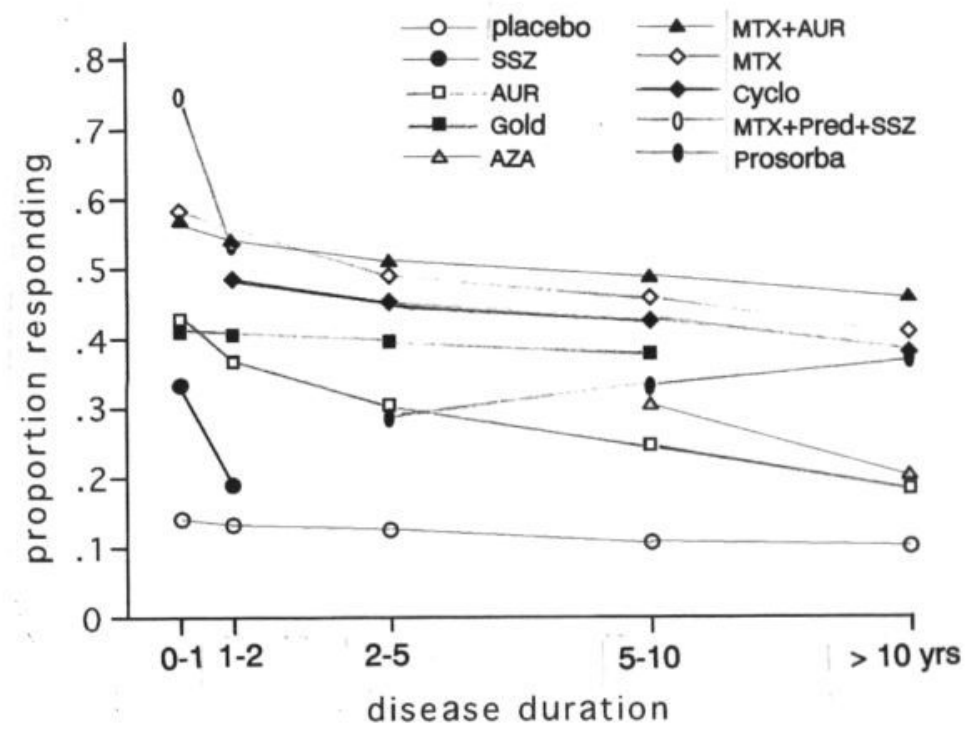

Figure 8.1: Proportion of patients in 14 trials having a response satisfying the ACR-20\% improvement criteria [6], within each combination of treatment and disease duration category. Treatments are placebo, sulphasalazine (SSZ), auranofin (AUR), sodium aurothiomalate (Gold), azathioprine (AZA), combination of methotrexate and auranofin (MTX + AUR), methotrexate (MTX), cyclosporin (Cyclo), combination of methotrexate, sulphasalazine, and step-down prednisolone (MTX + Pred + SSZ), and an immunoadsorption column, apheresis-based treatment containing Staphylococcal protein A (Prosorba). 
The difference between active treatment and placebo response rates was not affected by disease duration or the other factors. Table 8.4 shows response rates for active treatment and placebo-treated patients in all of the trials by categories of disease duration, Steinbrocker functional class, sex, and patient global assessment. The differences in response were generally $\sim 30 \%$ regardless of category, and did not vary significantly across the strata $(P>0.05$ in each case). Too few placebo-treated subjects had not had prior DMARD use $(n=6)$ to evaluate the response rates for this factor.

Table 8.4: ACR response rates for active treatment and placebo-treated patients within the strata of factors related to response

\begin{tabular}{|c|c|c|c|c|c|}
\hline & \multicolumn{2}{|c|}{ Active treatment } & \multicolumn{2}{|c|}{ Placebo treated } & \multirow[b]{2}{*}{$\begin{array}{l}\text { Active-placebo } \\
\text { difference, \% }\end{array}$} \\
\hline & No. & $\begin{array}{c}\mathrm{ACR} \\
\text { response, \% }\end{array}$ & No. & $\begin{array}{c}\mathrm{ACR} \\
\text { response, \% }\end{array}$ & \\
\hline Overall & 1178 & 43 & 262 & 10 & 33 \\
\hline \multicolumn{6}{|l|}{ By sex } \\
\hline Male & 371 & 46 & 72 & 13 & 31 \\
\hline Female & 807 & 41 & 190 & 8 & 33 \\
\hline \multicolumn{6}{|l|}{$\begin{array}{l}\text { By baseline patient's } \\
\text { global assessment }\end{array}$} \\
\hline$<5$ & 222 & 39 & 42 & 5 & 34 \\
\hline$\geq 5$ & 943 & 44 & 212 & 11 & 33 \\
\hline \multicolumn{6}{|c|}{ By Steinbocker functional class } \\
\hline I or II & 710 & 43 & 166 & 13 & 30 \\
\hline III & 194 & 38 & 96 & 4 & 34 \\
\hline \multicolumn{6}{|l|}{ By disease duration } \\
\hline$\leq 1$ year & 312 & 52 & 10 & 20 & 32 \\
\hline$>1, \leq 2$ years & 148 & 43 & 9 & 22 & 21 \\
\hline$>2, \leq 5$ years & 245 & 44 & 36 & 14 & 30 \\
\hline$>5, \leq 10$ years & 213 & 39 & 72 & 7 & 32 \\
\hline$>10$ years & 260 & 35 & 135 & 9 & 26 \\
\hline
\end{tabular}

We examined the response by disease duration for each of the core set components separately. For each core set measure, we found lower proportions of patients who improved by at least $20 \%$ with longer disease duration. The response in tender joint count and swollen joint count dropped off more steeply than did the response in other core set items (Figure 8.2), yet these 2 items, both of which must improve by at least $20 \%$ for the ACR improvement criteria to be satisfied, remained more likely to improve than did any of the other 5 items in each of the 5 intervals of disease duration. The drop-off in response with disease duration was fairly uniform across all components of the ACR core set. 


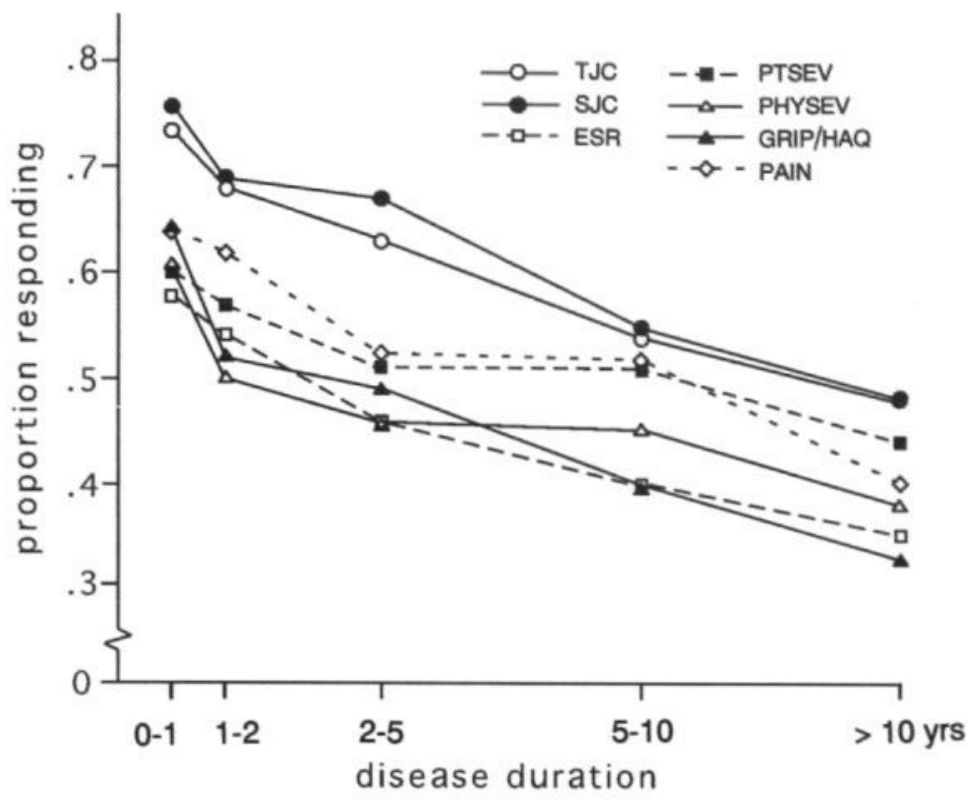

Figure 8.2: Proportion of patients in 14 trials improving by at least $20 \%$ in each of 7 ACR coreset items, within each disease duration category.

ACR core-set items are tender joint count (TJC), swollen joint count (SJC), erythrocyte sedimentation rate (ESR), patient's assessment of disease severity (PTSEV), physician's assessment of disease severity (PHYSEV), grip strength (in 11 trials) or health assessment questionnaire score (in 3 trials) (GRIP/HAQ), and patient's assessment of pain (PAIN). 


\section{Discussion}

The major findings of our analyses are that disease duration has a considerable impact on response to treatment, as do the Steinbrocker functional class and previous DMARD use. Understanding the magnitude of these effects and of the difference in response for male and female patients can be useful to both patients and physicians in anticipating the likelihood of response to treatment with any of these DMARDs. In these trials, we saw a net $5 \%$ greater response probability for male versus female patients and between Steinbrocker functional class II and functional class III patients, as well as a $10 \%$ greater response probability among patients who had not previously been treated with DMARDs.

Some effect of disease activity is to be expected in patients entering a clinical trial. We found a $5 \%$ difference in response for patients with high versus low baseline disease activity levels as measured using the patient global assessment. In part, this may represent regression to the mean, since the usual requirement of a certain level of disease activity for patients entering a clinical trial generally results in some improvement in disease activity during the trial, independent of any effects of medication. However, the effect of disease activity seen in these data was substantial, as large as the difference seen between male and female patients and between the 2 levels of disease severity.

A limitation of this study was the absence, from the trial data available to us, of information on rheumatoid factor, which may affect response to treatment $[22,23]$. In addition, only 3 trials had HAQ data available as a measure of physical function, with the remainder of trials providing grip strength data. It is possible that we could have found stronger results for the independent effects of physical function on response to treatment if a more comprehensive measure had been available in a greater number of trials.

A lesser limitation is that 3 of the trials whose data were included did not have 1 of the ACR core set measures available, specifically, the physician's global assessment. This meant that it was slightly more difficult for the patients in these trials to achieve the ACR response compared with patients in other trials. To satisfy the response criteria, the patients in these trials were required to improve by at least $20 \%$ in 3 of 4 items rather than 3 of the 5 available to a patient in whom all core set items had been measured. The trials affected were both of the MTX versus injectable gold trials and the larger of the 2 MTX versus AZA trials. The effects may be seen in Figure 8.1, where the response to injectable gold was consistently less than the response to cyclosporine or to MTX for each disease duration category. Similarly, the AZA response in these data was relatively low, being only slightly higher than that of patients with similar disease duration who were treated with AUR.

The disease duration effect was strongest in patients with a shorter disease duration. When the COBRA trial, with its very short disease duration, was not included in the multivariate analysis (using the 10-trial "all variable" data set), the disease duration effect, though still statistically significant, was attenuated $\left(\mathrm{OR}_{\mathrm{dj}} 0.74\right.$ per 15 years versus $O \mathrm{R}_{\mathrm{adj}} 0.63$ in the "all trial" data set). Also, there was no disease duration trend for the small number of Prosorba-treated participants, of whom more than $80 \%$ ( 39 of 48 ) had substantial disease duration of $\geqslant 5$ years. 
The most widely used treatments for RA are represented in our data, but we did not have trial data on major new agents for RA, especially those targeting tumor necrosis factor $\alpha$. It is not clear whether response rates also diminish with longer disease duration when these agents are used.

We found an $-30 \%$ difference between active and placebo treatment response that varied little across the strata of disease duration, Steinbrocker functional class, disease activity, and sex. An implication of this finding is that the distribution of patients in a trial with respect to levels of these factors should have little effect on the statistical power of the trial. Nevertheless, the interpretability of trial results in general would be enhanced by the explicit use of disease duration strata in particular. Many trials have already planned to include or exclude patients on the basis of disease duration, prior DMARD use, Steinbrocker functional class, or sex. When trials include patients in multiple strata, the presentation of trial results by these strata would facilitate comparisons of results across trials.

A problem with the interpretation of our finding of a reduced likelihood of response to treatment with greater duration of disease would arise if the disease duration effect were an artifact of the way response is measured. It could be that some components of the core set are less responsive than others for patients with greater disease duration, and thus arguably unsuited for use as part of a response criterion in longer-standing disease. In particular, it might be expected that the presence of fixed residual swelling of joints would reduce responsiveness of the swollen joint count, and that physical function might also be intrinsically less responsive in patients with longer-standing and more advanced disease. Our examination of the relationship between disease duration and response for each separate ACR core set item showed a fairly uniform drop-off in response with increasing disease duration. This suggests that there is a real decline in disease responsiveness with greater duration of disease, and that the decrease is not an artifact of measurement.

Patients with longer disease duration who enter clinical trials are likely to have prior DMARD exposure, and these DMARDs may not have worked well for them, otherwise it is unlikely that they would be participating in a clinical trial. Our data show independent effects of disease duration, disease severity, and prior DMARD exposure with respect to ACR response, which suggests that the patients who are least likely to respond are those who have long-standing, refractory disease.

In summary, we have found, from an analysis of patient-level data in a set of 14 clinical trials in RA, that patients with longer disease duration do not respond as well to treatment in RA as do those with earlier disease, and that female sex, prior DMARD use, and worse functional class also, simultaneously, reduce the likelihood of patient response. There is no evidence that the ACR improvement criteria are less valid in longer-standing disease, nor should the power of trials be adversely affected by any particular distribution of patients across the various strata of factors related to response. The implications for trial interpretation and for clinical expectations of patients, however, are that the response can be expected to vary according to which group a patient is in with respect to each of the variables of disease duration, sex, prior DMARD use, and disease severity. In particular, there is a window of opportunity for effective treatment of RA during the early stages of the disease. 


\section{References}

1. American College of Rheumatology Ad Hoc Committee on Cinical Guidelines. Guidelines for the management of rheumatoid arthritis. Arthritis Rheum 1996; 39:713-22.

2. Emery P, Salmon M. Early rheumatoid arthritis: time to aim for remission? Ann Rheum Dis 1995; 54:944-7.

3. Harris ED Jr. Rheumatoid arthritis: pathophysiology and implications for therapy. N Engl J Med 1990; 322:1277-89.

4. van der Heide A, Remme CA, Hofman DM, Jacobs JWG, Bijlsma JWJ. Prediction of progression of radiologic damage in newly diagnosed rheumatoid arthritis. Arthritis Rheum 1995; 38:1466-74.

5. Egsmose C, Lund B, Borg G, et al. Patients with rheumatoid arthritis benefit from early 2nd line therapy: 5 year followup of a prospective double-blind placebo controlled study. J Rheumatol 1995; 22:2208-13.

6. Felson DT, Anderson JJ, Boers M, et al. American College of Rheumatology preliminary definition of improvement in rheumatoid arthritis. Arthritis Rheum 1995; 38:727-35.

7. Fries JF, Spitz PW, Kraines RG, Holman HR. Measurement of patient outcome in arthritis. Arthritis Rheum 1980; 23:137-45.

8. Steinbrocker O, Traeger CH, Batterman RC. Therapeutic criteria in rheumatoid arthritis. JAMA 1949; 140:659-62.

9. Chernoff MC, Wang M, Anderson JJ, Felson DT. Problems and suggested solutions in creating an archive of clinical trials data in rheumatoid arthritis: an example of methotrexate trials in rheumatoid arthritis. Control Cin Trials 1995; 16:342-55.

10. Weinblatt ME, Coblyn JS, Fox DA, et al. Efficacy of low-dose methotrexate in rheumatoid arthritis. NEngl J Med 1985; 312:818-22.

11. Furst DE, Koehnke R, Burmeister LF, Kohler J, Cargill I. Increasing methotrexate effect with increasing dose in the treatment of resistant rheumatoid arthritis. J Rheumatol 1989; 16:313-20.

12. Williams HJ, Willkens RF, Samuelson CO Jr, et al. Comparison of low-dose oral pulse methotrexate and placebo in the treatment of rheumatoid arthritis: a controlled clinical trial. Arthritis Rheum 1985; 28:721-30.

13. Weinblatt ME, Kaplan H, Germain BF, et al. Low-dose methotrexate compared with auranofin in adult rheumatoid arthritis: a thirty-six-week, double-blind trial. Arthritis Rheum 1990; 33:330-8.

14. Williams HJ, Ward JR, Reading JC, et al. Comparison of auranofin, methotrexate, and the combination of both in the treatment of rheumatoid arthritis: a controlled clinical trial. Arthritis Rheum 1992; 35:259-69.

15. Suarez-Almazor ME, Fitzgerald A, Grace M, Russell AS. A randomized controlled trial of parenteral methotrexate compared with sodium aurothiomalate (myochrysine) in the treatment of rheumatoid arthritis. J Rheumatol 1988; 15:753-6.

16. Morassut P, Goldstein R, Cyr M, Karsh J, McKendry RJR. Gold sodium thiomalate compared to lowdose methotrexate in the treatment of rheumatoid arthritis - a randomized double-blind 26-week trial. J Rheumatol 1989; 16:302-6.

17. Hamdy H, McKendry RJR, Mierins E, Liver JA. Low-dose methotrexate compared with azathioprine in the treatment of rheumatoid arthritis: a twenty-four-week controlled clinical trial. Arthritis Rheum 1987; 30:361-8.

18. Bell M, Chang C.H, Keystone EC, Urowitz MB, Gladman D, Ching CF. A double-blind randomized controlled trial of azathioprine and methotrexate in the treatment of rheumatoid arthritis. Arthritis Rheum 1988; 31 suppl 4:S114.

19. Tugwell $P$, Pincus T, Yocum D, et al. Combination therapy with cyclosporin and methotrexate in severe rheumatoid arthritis. NEngl J Med 1995; 333:137-41. 
20. Boers M, Verhoeven AC, Markusse HM, et al. Randomised comparison of combined step-down prednisolone, methotrexate and sulphasalazine with sulphasalazine alone in early rheumatoid arthritis. Lancet 1997; 350:309-18.

21. Felson DT, LaValley MP, Baldassare AR, et al. The Prosorba column for treatment of refractory rheumatoid arthritis: a randomized, double-blind sham-controlled trial. Arthritis Rheum 1999; 42:2153-9.

22. Carson DA, Pasquali JL, Tsoukas CD, et al. Physiology and pathology of rheumatoid factors. Springer Semin Immunopathol 1981; 4:161-79.

23. Koopman WJ, Schrohenloher RE. Rheumatoid factor. In: Usinger PD, Zvaifler NJ, Erlich GE, eds. Rheumatoid arthritis: etiology, diagnosis, management. Philadelphia: Lippincott; 1985. p 217-42. 


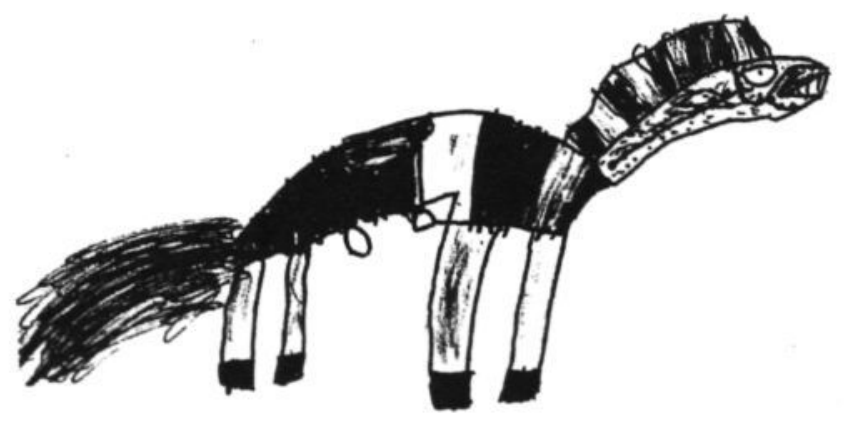




\section{CHAPTER 9}

\section{RESPONSIVENESS OF}

THE CORE SET, RESPONSE CRITERIA AND UTILITY MEASURES IN EARLY RHEUMATOID ARTHRITIS

Arco Verhoeven, Maarten Boers, Sjef van der Linden

Annals of the Rheumatic Diseases 2000; 59: 966-74. 


\section{Responsiveness of the Core Set, Response Criteria and Utility Measures in Early Rheumatoid Arthritis}

\section{Abstract}

Objective: Validation of responsiveness and discriminative power of the World Health Organisation/ International League of Associations for Rheumatology (WHO/ILAR) core set, the American College of Rheumatology (ACR) and European League Against Rheumatism (EULAR) criteria for improvement/response, and other single and combined measures (indices) in a trial with early rheumatoid arthritis (RA).

Methods: Ranking of measures by response (standardised response means and effect sizes) and between-group discrimination (unpaired $t$ test and $\chi^{2}$ values) at two time points in the COBRA study. This study included 155 patients with early RA randomly allocated to two treatment groups with distinct levels of expected response: combined treatment, high response; sulphasalazine treatment, moderate response.

Results: At week 16, standardised response means of core set measures ranged between 0.8 and 3.5 for combined treatment and between 0.4 and 1.2 for sulphasalazine treatment ( $95 \%$ confidence interval \pm 0.25 ). Performance of patient oriented measures (for example, pain, global assessment) was best when the questions were focused on the disease. The most responsive single measure was the patient's assessment of change in disease activity, at 3.5. Patient utility, a generic health status measure was moderately (rating scale) to poorly (standard gamble) responsive. Response means of most indices (combined measures) exceeded 2.0, the simple count of core set measures improved by $20 \%$ was most responsive at 4.1 . Discrimination performance yielded similar but not identical results: best discrimination between treatment groups was achieved by the EULAR response and ACR improvement criteria (at $20 \%$ and other percentage levels), the pooled index, and the disease activity score (DAS), but also by the Health Assessment Questionnaire (HAQ) and grip strength.

Conclusions: Responsiveness and discrimination between levels of response are not identical concepts, and need separate study. The WHO/ILAR core set comprises responsive measures that discriminate well between different levels of response in early RA. However, the performance of patient oriented measures is highly dependent on their format. The excellent performance of indices such as the ACR improvement and EULAR response criteria confirms they are the preferred primary endpoint in RA clinical trials. 


\section{Introduction}

Many end point measures are available to assess treatment efficacy in rheumatoid arthritis (RA). The OMERACT consensus on end point measures in RA facilitates comparison of results from different trials in treatment evaluation [1]. The OMERACT initiative has called for further validation of the measures included in the World Health Organisation/International League of Associations for Rheumatology (WHO/ILAR) core set (also known as American College of Rheumatology (ACR) core set [2] and combined measures (indices) such as improvement and response criteria $[3,4]$. To determine the applicability of a measure in a certain setting, the OMERACT filter has been proposed, containing three elements: truth, discrimination, and feasibility [5]. The first two elements capture classic validity concepts. The topic of this study is discrimination. To be discriminative in trials, a measure has to detect clinically relevant change; moreover, it has to detect clinically relevant differences in change between treatment groups. Highly responsive measures are preferred because they allow clinical trials to be done with fewer patients, and also because they facilitate detection of small - but potentially important differences in treatment effect [6]. For the clinician, applying responsive measures in patient care will allow better tailoring of individual treatment. However, individual patient care may require another selection of measures than those used in trials: for example, assessment of morning stiffness and disease activity in the feet joints remain useful in the clinic despite their exclusion from the core set [7].

As every measure is bound to pick up some noise together with the intended signal, its responsiveness is determined by the ratio of treatment effect to its variability (signal-to-noise ratio). Two classes of responsiveness statistics can be distinguished: the first is based on measurement of change in the course of a therapeutic intervention with known efficacy (external criterion, gold standard); the second is directed at the correlation of change in the tested measure with change in a "criterion measure". However, as this last class of responsiveness statistics is based on the variability between subjects, regardless of whether group changes occur, they yield little information about the ability of a measure to detect treatment effects [8].

The purpose of this study was to validate further the responsiveness of the core set, of the ACR improvement [3] and European League Against Rheumatism (EULAR) response criteria [4], and of other single measures and indices with data from a recent trial. The COBRA study [9] (Dutch acronym: COmbinatietherapie Bij Reumatoïde Artritis) was a randomised controlled trial in patients with early RA that showed excellent clinical response, low toxicity, and less progression of radiographic joint damage with treatment of combined step-down prednisolone, methotrexate and sulphasalazine, compared with treatment with sulphasalazine alone. The trial allowed us to create one "gold" and one "silver" standard for relevant response against which to validate performance of end point measures: we proposed the hypothesis a priori that patients in the combination group would on average show large and, certainly, relevant improvements at week 16 owing to the corticosteroid pulse (gold standard). Also, we assumed on the basis of the well known effects of sulphasalazine on disease activity that patients in the sulphasalazine-only group would also show relevant improvements, but to a lesser degree (silver standard). 
We then ranked the end point measures and indices used in the COBRA study by their relative responsiveness, and also by their ability to discriminate between the (changes in the) treatment groups. Ultimately, this discriminatory ranking yields the most relevant results. However, one must realise that this ranking is post-hoc: as the difference in response between the groups was the primary study question of the trial, the presence and extent of such a difference was unknown before the start of the trial.

\section{Patients and methods}

\section{The COBRA study}

The COBRA study [9] was a 56 week clinical trial that randomly assigned 155 patients with RA (ACR criteria [10]), aged 23-70, to one of two treatments. All patients had early, active disease (diagnosis $<2$ years). No prior treatment with disease-modifying anti-rheumatic drugs, apart from anti-malarials, was allowed. One group was treated with a combination of sulphasalazine, methotrexate, and, initially, high dose oral prednisolone, the other group with sulphasalazine and double placebo. The prednisolone dose was $60 \mathrm{mg}$ daily in the first week, tapered in weekly steps to the maintenance dose of $7.5 \mathrm{mg}$ in week 7. Prednisolone and methotrexate (or the placebos) were tapered and stopped after weeks 28 and 40, respectively, while sulphasalazine was continued.

\section{Core set measures}

A broad variety of endpoints was assessed, including all disease activity measures of the WHO/ILAR core set [1]. This comprises tender and swollen joint count (68 and 48 joints, respectively [11]), pain, assessor's and patient's global assessment (on $10 \mathrm{~cm}$ visual analogue scales [VAS], acute phase reactant (that is, erythrocyte sedimentation rate, Westergren method [ESR] or $C$ reactive protein [CRP]), and physical function (by Health Assessment Questionnaire; Dutch HAQ[12,13]).

\section{Non-core set measures}

Non-core set measures included other joint counts and score such as the Ritchie index, grip strength (by vigorimetry; Martin, Tottlingen, Germany, mean of medians of three measurements in both hands [14]), Arthritis Impact Measurement Scale (AIMS) [15] - a modified and validated Dutch version with scales for mobility, pain, and self-efficacy, and the McMaster Toronto Arthritis patient preference questionnaire (MACTAR) [16]. The MACTAR is an instrument that follows improvement in five impaired activities, elicited and ranked in priority by the patient at baseline, together with changes in quality of life, psychological, social, and emotional wellbeing. Its scores increase as functional ability improves and vary from 11 (maximum deterioration) to 47 (maximum improvement). In its original format the baseline scores differ from the follow-up scores because items inquiring about change are not included. To make these scores directly comparable mock change items were added at baseline and scored as "unchanged". To compare the responsiveness and discriminatory power of different formats of patient global assessment (see Appendix), two items from the MACTAR interview (change in disease activity by seven point Likert scale, and physical function by two questions with a six point scale), and a question on the actual state of 
disease activity (from a monitoring questionnaire; five point Likert scale) were evaluated together with the patient's global assessment of health indicated on a $10 \mathrm{~cm}$ VAS.

\section{Generic measures}

Whereas these disease-specific measures are sensitive to clinical change in RA, other - generic - measures yield a broader picture of patients' health status and allow comparison across a range of conditions [17]. Utility served as the central concept of generic measures in the COBRA study [18]. Utility is a single value or preference that patients assign to a particular health state. This value is expressed on a scale ranging from 1 (perfect health) to 0 (death) and takes into account both the positive treatment effects and negative side-effects. The rating scale and standard gamble methods assessed utility; the rating scale method derives utilities directly by asking the patients to place health states on a thermometer scale (that is, vertical VAS), the standard gamble method derives utilities from the patients' responses to decision situations under risk $[19,20]$. Utility scores were assessed at baseline, and weeks 28 and 56.

\section{Indices}

Various indices (that is, composites from several measures) were assessed in the COBRA study (Table 9.1). In fact, a pooled index of five measures (composite measure to reflect each patient's standardized improvement) - was the assigned primary outcome. Pooling is a validated method to increase responsiveness of separate measures [21]. To obtain a patient's pooled index score, the standardised change score was calculated by dividing change in one measure by its pooled standard deviation of change per treatment group at week 28. This procedure was repeated for five measures; the pooled index is the mean of standardised scores. Finally, a constant was added so that all index values started with a zero value at baseline. To obtain pooled index values for other time points than week 28 , change scores at that point were divided by the same factor (the SD of change of the measure at week 28). The trial was designed before the conception of the WHO/ILAR core set [1]. Recommendations at that time were to select five measures for maximum sensitivity to change [22]: tender joint count, global assessment by an independent assessor, grip strength, and MACTAR. The original Disease Activity Score (DAS) [23] was also calculated. This index contains the Ritchie tender joint index, swollen joint count,, and patient's global assessment

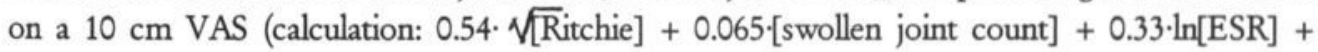
0.072 [patient's global].

\section{Remission, improvement and response}

Improvement in individual patients was also assessed in several ways: the ACR [24] and DAS remission criteria [25], ACR improvement [3] and EULAR response [4] criteria, and count of improved core set measures [26] (Table 9.1). Because fatigue was not measured in the trial "probable remission" described instances in which a patient would be in remission when absence of fatigue was assumed. Modified ACR improvement criteria and counts of improved core measures were also calculated with improvement thresholds varying from 0 to $70 \%$ (Table 9.1). To calculate percentage improvement, values were recoded where necessary to ensure that all scales decreased on improvement. 


\section{Follow-up}

Initially, grip strength, and patient's assessment of disease activity (five point Likert scale) were registered weekly by research nurses, later at least every four weeks. All other reported assessments - with exception of utilities - were made at baseline and at weeks 16, 28, (40, and 56) by trained independent assessors who contacted the patients only at these times. This way, the assessors were unaware of effects of high-dose prednisolone during the first six weeks of the protocol. Utility scores were assessed biannually; thus for these measures only 28 week follow-up measures are reported.

\section{Statistical analysis}

All analyses were based on intention to treat: only five patients $(3 \%$, all in the sulphasalazine group) were lost to follow-up before week 56 of the trial. The primary statistic of responsiveness was the standardised response mean (SRM): mean observed change from baseline divided by the standard deviation of this change [27]. The effect size (ES) [28]: the mean change from baseline divided by the standard deviation (SD) of baseline scores was also calculated. Confidence intervals of SRMs were calculated with the assumption that its distribution is approximately Gaussian with mean zero and SD of one over the square root of the sample size [29]. From the confidence intervals, statistical difference between SRMs could be evaluated. With no correction made for multiple comparison these findings are solely informative. Most evaluated variables are on an ordinal rather than interval or ratio scale level. However, as the underlying phenomenon (disease activity) is on an interval scale, these measures can be analysed parametrically, if the sample size is large enough, as in the COBRA study database (central limit theorem).

Ceiling and floor effects may impair responsiveness when baseline values are found on the upper and lower end of the scale. We arbitrarily defined these extremes at the upper and lower one-sixth of the scale (comparable to baseline HAQ scores $<0.5$ or $>2.5$ ) and analysed the variables in the core set.

As stated in the introduction, statistics based on change from therapeutic interventions need a priori confidence that the treatment is effective - that is, that the mean improvement in the treated group is relevant. The a priori criterion was a large change from baseline in the combinedtreatment group at 16 weeks ("gold standard"); less change from baseline was expected in the group treated with sulphasalazine ("silver standard"). This proved to be true, though the change reached at 28 weeks was slightly larger, especially in the sulphasalazine-only group. Thus, the combined-treatment group SRM at week 16 was the primary statistic of responsiveness to form a league table for responsiveness. The SRMs in the sulphasalazine group can be used to assess the ability of a measure to detect smaller - but still meaningful - changes, or changes occurring in a smaller proportion of the treatment group. 
To indicate the discriminative power between groups the unpaired Student's $t$ test values are reported. $\chi^{2}$ values reflect between-group contrast (that is, discriminative power) in the nominal variables: improvement and remission criteria. Because the primary study question of the CORRA trial concerned contrast between treatment groups, this contrast could not be an a priori criterion such as improvement in the combined-treatment group as outlined above [30]. Consequently, the ranking based on discrimination must be interpreted with caution. The 28 week data are included to allow further exploration of trends in responsiveness and discriminatory power.

Table 9.1: Definition of indices.

Index [reference]

Pooled index (measures A, B, C...) [21]

Disease Activity Score (DAS) [23]

ACR remission [24]

COBRA, "probable" ACR remission [9]

DAS remission [25]

ACR improvement (20\%) [3]

Modified ACR improvement (\# \%)*

No of core measures improved (\# \%)* [26]

EULAR response [4]
Calculation

mean of [change in measure $A / S D_{\text {change }} A$; change in measure $\mathrm{B} / \mathrm{SD}_{\text {change }} \mathrm{B}$;

change in measure $\mathrm{C} / \mathrm{SD}_{\text {change }} \mathrm{C}$; etc.]

$0.54 \mathrm{~V}$ [Ritchie index] +0.065 [swollen joint count] $+0.33 \ln [$ ESR] +0.072 [patient global].

5 out of 6 , for $\geq 2$ months: morning stiffness $\leq 15 \mathrm{~min}$, no joint pain, no joint pain on exam, no joint swelling, ESR $<30 \mathrm{~mm}$ (men, $<20 \mathrm{~mm} / \mathrm{h}$ ), and no fatigue

4 out of 5 , absence of fatigue assumed (not assessed) DAS $\leq 1.6$

improvement by at least $20 \%$ in tender joint count and swollen joint count plus in at least 3 out of the remaining 5 core set measures: acute phase reactant, physical function, physician's global assessment, patient's global -, and pain

Improvement by at least $20 \%$ in tender joint count and swollen joint count plus in at least 3 out of the remaining 5 core set measures

count of core set measures improved by at least \# \% 'good': improvement in DAS $>1.2$ and final DAS $\leq 2.4$

'moderate' : not meeting criteria for "good", but improvement in DAS $>0.6$ and final DAS $\leq 3.7$

'non': remaining

* $\#$ \% = thresholds varying from 0 to $70 \%$. 


\section{Results}

The combined-treatment group included 76 and the sulphasalazine group 79 patients. The groups were balanced in important demographic and prognostic variables $[9,18]$. At week 16 the mean improvement in terms of the pooled index was 1.4 for the combined-treatment group and 0.7 for the sulphasalazine group $(P<0.0001)$. At week 28 these values were $1.5 \tau 0.8(P<$ 0.0001 ). In the combined-treatment group rates and rapidity of $A C R 20,50$, and 70 improvement were similar to those reported in recent trials on anti-tumour necrosis factor (antiTNF) treatment (see below).

At week 16, most measures indicated large improvement from baseline in both groups (SRM: 0.4-4.1, ES: 0.3-3.2), and all measures except patient's global assessment of health (VAS) significantly distinguished combined treatment from sulphasalazine (Table 9.2, Figure 9.1). Statistics of responsiveness were larger in the combined-treatment group than in the sulphasalazine group, confirming a priori assumptions of greater improvement in this group. The relative responsiveness ranking of measures was similar in both groups, suggesting the ranking is stable over a broad range of relevant response. However, in the sulphasalazine-only group the absolute differences in responsiveness between measures were less, in proportion to the overall decreased response. All indices (that is, pooled index, MACTAR, DAS and count of improved core set measures) were - in both treatment groups - considerably more responsive than single core set measures such as tender joint count. The only exception to this was the highly responsive single item patient's assessment of change in disease activity on a seven point Likert scale. The responsiveness of most single measures was satisfactory but not equal (for example, high responsiveness for pain and ESR, lower for tender joint count and CRP). A confidence interval smaller than 0.5 around the SRM estimates indicates that a difference between SRMs of 0.35 or greater would be significant when tested at the two sided 0.05 level. The results at week 28 were generally similar (Table 9.3, Figure 9.1).

The format of patient assessment of disease activity, physical impairment, and global wellbeing strongly influenced responsiveness (for a description of the formats see Appendix). The item in the MACTAR interview that asked for change of disease activity (seven point Likert scale) proved to be most responsive, patient's global assessment of health indicated on a VAS, least responsive. MACTAR, HAQ, some AIMS subscales and single item patient global assessment of physical function were not equally responsive. The utility rating scale showed responsiveness close to the patient global assessment of disease activity, whereas utility measured by standard gamble was the least responsive of all measures.

Analyses on floor and ceiling effects showed that, of the core set variables, ESR and tender and swollen joint count were vulnerable to a certain degree of floor effect, with respectively 17, 15, and $15 \%$ of the patients in the lowest one sixth segment of the scale. Global and pain assessments, and also the HAQ had fewer patients that scored at the extremes of the scale. 
Table 9.2: Indices of responsiveness at week 16 of follow-up, for each treatment group, ordered by standardised response mean (SRM) of the combination group

$\begin{gathered}\text { combined treatment } \\ (n=75)\end{gathered}$
$\begin{gathered}\text { mean } \\ \text { change } \mathrm{SE}_{\Delta} \mathrm{SRM} \quad \mathrm{ES}\end{gathered}$

$\frac{\begin{array}{c}\text { sulphasalazine } \\ (n=79)\end{array}}{\begin{array}{c}\text { mean } \\ \text { change SE } \mathrm{SE}_{\Delta} \mathrm{SRM} \text { ES }\end{array}}$

$t$

value
Count core set measures $\geq 20 \%$ improved

Change in disease activity

by patient, 7 point Likert

Pooled index

composite measure

MACTAR

interview

Count core set measures $\geq 50 \%$ improved

Disease Activity Score (DAS) AIMS composite measure

pain scale

Health Assessment

Questionnaire

Pain assessment by patient, VAS

Disease activity ESR by patient, 5 point Likert

(mm/1h)

Swollen joint count ARA 48

Grip strength $(\mathrm{kPa})$

Count core set measures $\geq 70 \%$ improved

Global assessment by observer, VAS

Physical function by patient, 6-point Likert

Tender joint count ARA 68

Ritchie Arthritis Index

CRP tender joints

(mg/L)

AIMS mobility scale

Global assessment AIMS by patient, VAS

self-efficacy scale $\begin{array}{lllllllllll}5.9 & 0.2 & 4.1 & - & 4.1 & 0.2 & 2.2 & - & & 5.7\end{array}$

$\begin{array}{lllllllllll}2.6 & 0.1 & 3.5 & - & 1.7 & 0.2 & 1.2 & - & 5.3\end{array}$

$\begin{array}{llllllllllll} & 1.4 & 0.1 & 2.4 & - & 0.7 & 0.1 & 1.1 & - & & 6.5\end{array}$

$\begin{array}{lllllllll}12 & 0.6 & 2.2 & 3.2 & 8.5 & 0.8 & 1.3 & 2.1 & 3.7\end{array}$

$\begin{array}{lllllllllll}4.6 & 0.2 & 2.2 & - & & 2.4 & 0.3 & 1.0 & - & & 6.0\end{array}$

$\begin{array}{lllllllll}-2.1 & 0.1 & 1.9 & 1.9 & -1.2 & 0.1 & 1.0 & 1.2 & 4.9\end{array}$

$\begin{array}{lllllllll}-8.4 & 0.5 & 1.8 & 2.3 & -5.7 & 0.5 & 1.2 & 1.6 & 3.6\end{array}$

$\begin{array}{lllllllll}-1.1 & 0.1 & 1.5 & 1.5 & -0.4 & 0.1 & 0.8 & 0.6 & 6.2\end{array}$

$\begin{array}{lllllllll}-3.3 & 0.3 & 1.5 & 1.6 & -1.8 & 0.3 & 0.7 & 0.8 & 4.0\end{array}$

$\begin{array}{lllllllll}-1.7 & 0.1 & 1.5 & 1.6 & -1.1 & 0.1 & 0.9 & 1.1 & 2.7\end{array}$

$\begin{array}{lllllllll}-41 & 3 & 1.4 & 1.2 & -23 & 3 & 0.9 & 0.7 & 4.1\end{array}$

$\begin{array}{lllllllll}-9 & 1 & 1.4 & 1.0 & -5 & 1 & 0.7 & 0.6 & 4.2\end{array}$

$\begin{array}{lllllllll}22 & 2 & 1.3 & 1.5 & 8 & 1 & 0.6 & 0.4 & \end{array}$

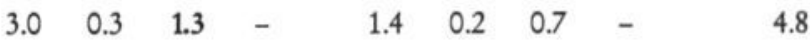

$\begin{array}{lllllllll}-30 & 3 & 1.3 & 1.3 & -14 & 3 & 0.6 & 0.7 & 4.3\end{array}$

$\begin{array}{lllllllll}1.8 & 0.2 & 1.3 & 1.7 & 1.3 & 0.2 & 0.9 & 1.2 & 2.2\end{array}$

$\begin{array}{lllllllll}-14 & 1 & 1.2 & 1.0 & -6 & 1 & 0.5 & 0.4 & 4.1\end{array}$

$\begin{array}{lllllllll}-10 & 1 & 1.2 & 1.0 & -7 & 1 & 0.8 & 0.6 & 2.5\end{array}$

$\begin{array}{lllllllll}-33 & 4 & 0.9 & 0.8 & -18 & 5 & 0.5 & 0.5 & 2.2\end{array}$

$\begin{array}{lllllllll}5.3 & 0.7 & 0.9 & 1.0 & 2.1 & 0.5 & 0.5 & 0.3 & 3.9\end{array}$

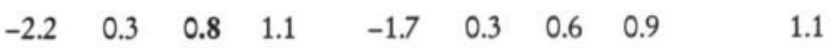

$\begin{array}{lllllllll}5.1 & 0.8 & 0.8 & 0.9 & 2.4 & 0.6 & 0.4 & 0.4 & 2.7\end{array}$

$\mathrm{SE}_{\Delta}=$ standard error of change; $\mathrm{SRM}=$ standardized response mean; ES = effect size; $t$ value unpaired (i.e., between group). SRM 95\% confidence intervals have a width of $<0.5$ around the listed value, between-SRMs differences $\geq 0.35$ are significant (two sided $P<0.05$, no correction for multiple comparison) 
Table 9.3: Indices of responsiveness at week 28 of follow-up, for each treatment group*

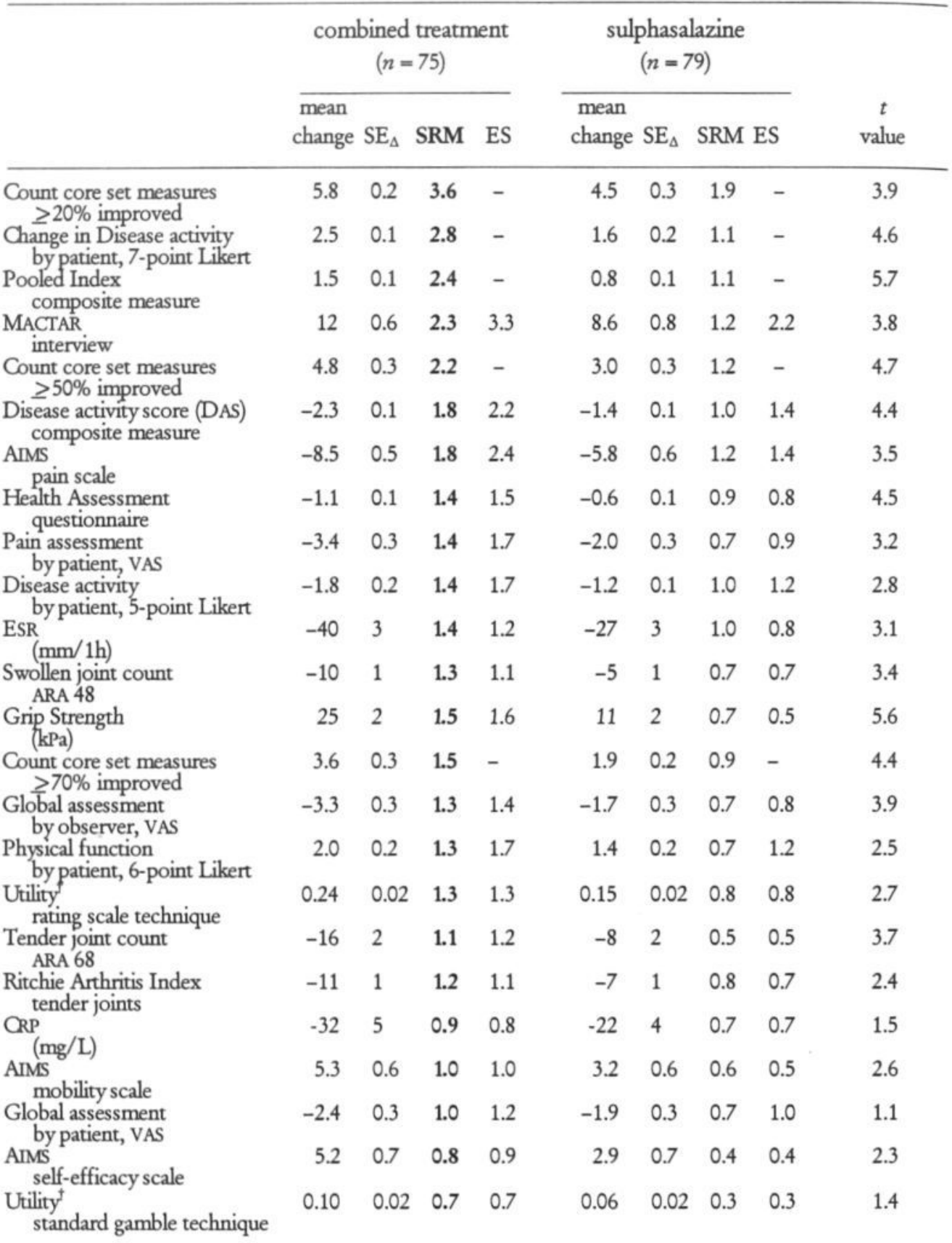

* Abbreviations, see Table 9.2. SRM 95\% confidence intervals have a width of $<0.5$ around the listed value, between-SRMs differences $\geq 0.35$ are significant (two sided $P<0.05$, no correction for multiple comparison). $†$ Utilities were only assessed at week 28. 
The ranking for between-group discrimination showed interesting trends. This is best seen in Figure 9.1: highest $t$ values (that is, most discriminative power) were found for pooled index, count of core set set measures improved by $50 \%$, DAS, HAQ but, also, grip strength. Between the two assessments a catch-up effect is seen in the sulphasalazine group: whereas improvements in the combination group were already maximum at week 16 , the sulphasalazine group improved further between week 16 and week 28, resulting in a smaller between-group difference (and thus a smaller $t$ value).

At 16 weeks, ACR 20\% improvement and EULAR response criteria showed large $\chi^{2}$ values, consistent with significant differences in response between treatment groups (Table 9.4). The discriminatory performance of these criteria ranks high among all the measures tested (Figure 9.4): based on $P$ values a $\chi^{2}$ value of 8 roughly corresponds with a $t$ value of 3 ; similarly, a $\chi^{2}$ value of 12 corresponds with a $t$ value of 4 , and a $\chi^{2}$ value of 25 with a $t$ value of 5 . At week 28 , the differences between the groups were smaller. At week 16 , modification of the percentage value in the ACR improvement criterion between $0 \%$ (no improvement, no worsening) and $50 \%$ did not change its discriminatory capacity, at week 28 , this was also true for the $70 \%$ cut off point (Table 9.4). The ACR and DAS remission criteria did not show a significant between-group difference.

Table 9.4: Discriminative ability of the preliminary ACR improvement criteria at different thresholds, EULAR response criterion, ACR and DAS remission criteria to distinguish between two treatment groups at two moments of follow-up

\begin{tabular}{|c|c|c|c|c|c|c|c|}
\hline \multirow[b]{2}{*}{ Criterion } & \multicolumn{4}{|c|}{ week 16} & \multicolumn{3}{|c|}{ week 28} \\
\hline & \multicolumn{2}{|c|}{ improved * } & \multirow[t]{2}{*}{$\chi^{2}$} & \multirow[t]{2}{*}{$P$} & improved * & \multirow[t]{2}{*}{$\chi^{2}$} & \multirow[t]{2}{*}{$P$} \\
\hline \multicolumn{4}{|l|}{ ACR improvement } & & & & \\
\hline O\% threshold & $88 v$ & $63 \%$ & 12.9 & .0003 & $82 u \quad 62 \%$ & 7.3 & .007 \\
\hline $10 \%$ threshold & $78 u$ & $44 \%$ & 18.0 & $<.0001$ & 74 us $49 \%$ & 9.7 & .002 \\
\hline $20 \%$ threshold & $72 v s$ & $32 \%$ & 25.7 & $<.0001$ & 72 ขs $49 \%$ & 8.6 & .003 \\
\hline $30 \%$ threshold & $64 v$ & $24 \%$ & 25.7 & $<.0001$ & 67 ws $39 \%$ & 12.1 & .0005 \\
\hline $40 \%$ threshold & $58 \tau s$ & $16 \%$ & 28.6 & $<.0001$ & 55 us $30 \%$ & 9.8 & .002 \\
\hline $50 \%$ threshold & $43 \tau s$ & $14 \%$ & 16.6 & $<.0001$ & 49 ขs $27 \%$ & 8.1 & .004 \\
\hline $60 \%$ threshold & $26 u s$ & $8 \%$ & 9.7 & .002 & 37 us $22 \%$ & 4.4 & .04 \\
\hline $70 \%$ threshold & $16 \tau$ & $6 \%$ & 3.6 & .06 & 29 vs $10 \%$ & 8.8 & .003 \\
\hline \multirow{3}{*}{$\begin{array}{l}\text { EULAR response; } \\
\text { good + moderate } \\
\text { good }\end{array}$} & & & \multirow[t]{3}{*}{20.2} & \multirow[t]{3}{*}{$<.0001$} & & \multirow[t]{3}{*}{13.4} & \multirow[t]{3}{*}{.001} \\
\hline & 86 vs & $56 \%$ & & & 86 vs 63\% & & \\
\hline & $37 \tau$ & $15 \%$ & & & 47 vs $24 \%$ & & \\
\hline \multicolumn{8}{|l|}{ ACR remission } \\
\hline 'probable' & $12 v s$ & $6 \%$ & 1.4 & .23 & 21 us $11 \%$ & 2.7 & .10 \\
\hline \multicolumn{8}{|l|}{ DAS remission } \\
\hline (DAS <1.6) & $12 \tau$ & $9 \%$ & 0.4 & .54 & 17 us $10 \%$ & 1.6 & .20 \\
\hline
\end{tabular}

*; combined-treatment $(n=76)$ vs sulphasalazine treatment $(n=79)$ group. 


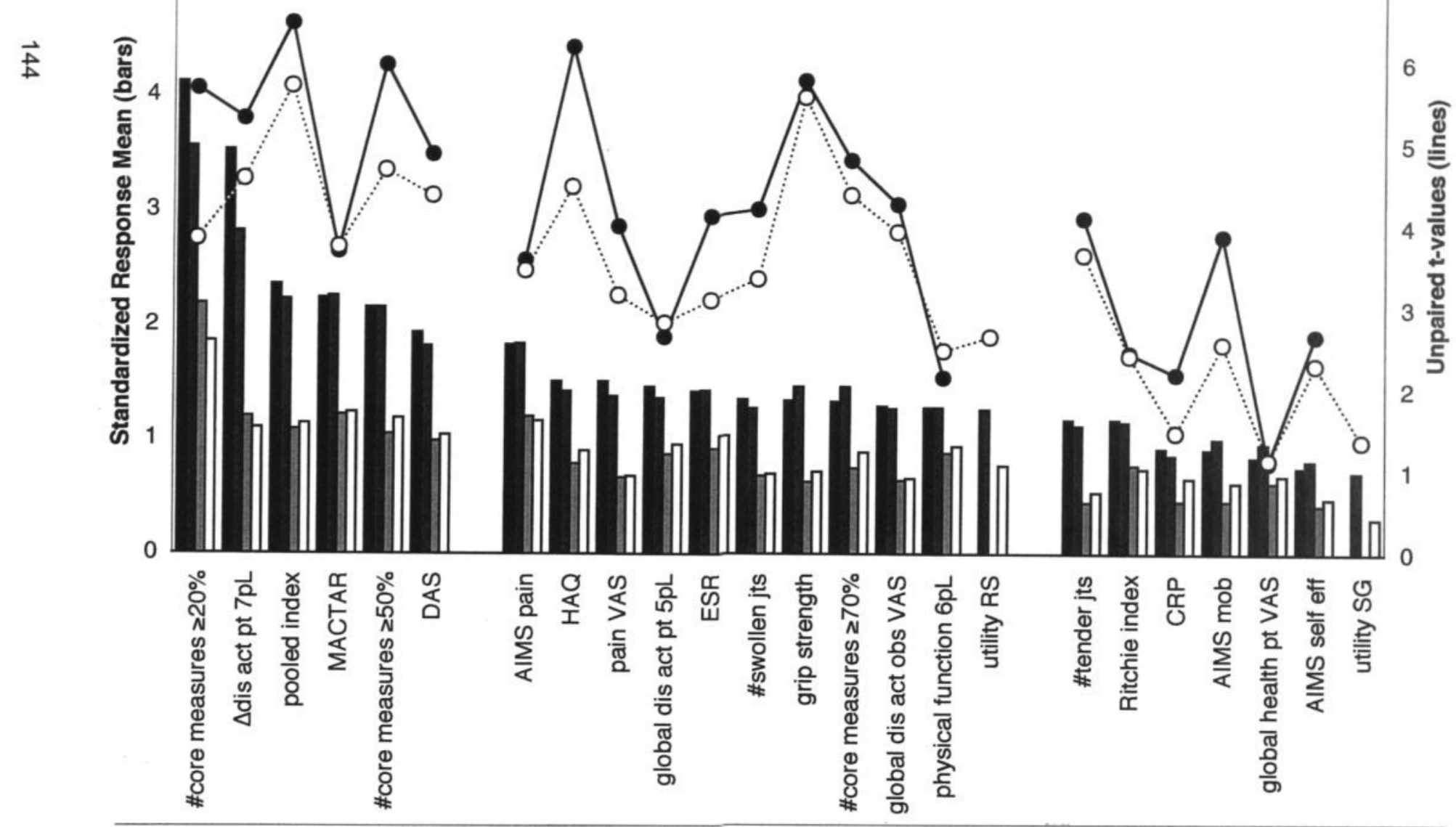

Figure 9.1: Comparison of responsiveness and discrimination performance of end points.

The order of the measures evaluated corresponds with that of Table 9.3. Dark bars: standardised response means of the combined-treatment group at weeks 16 (black) and 28 (dark grey). Light bars: SRMs of the sulphasalazine group at 16 (light grey) and 28 (white) weeks. Dots: unpaired $t$ values of the between-group comparison at 16 (black) and 28 weeks (white). $\mathrm{pL}=$ points Likert scale; MACTAR $=$ McMaster Toronto Arthritis patient preference questionnaire; $\mathrm{DAS}=$ disease activity score; $\mathrm{AIMS}=$ arthritis impact measurement scale; $\mathrm{HAQ}=$ health assessment questionnaire; $\mathrm{VAS}=$ visual analogue scale; $\mathrm{ESR}=$ erythrocyte sedimentation rate; $C R P=\mathrm{C}$ reactive proteine; $\mathrm{RS}=$ rating scale; $\mathrm{SG}=$ standard gamble. 


\section{Discussion}

This study is the first independent confirmation of the responsiveness of the WHO/ILAR core set measures and response criteria in a trial of early RA patients. In addition, it lends strong support to the use of other indices in such a trial. The conclusions are strong because they are based on the findings in two groups of patients with a high and moderate level of expected response. They extend the validity of both the core set and the ACR response criteria, because these had initially been selected, designed, and tested mainly in the setting of placebo controlled studies.

The fact that indices are more responsive than most single measures is not surprising, as combining measures (or items in a questionnaire) reduces scatter. Even a simple count of improved core set outcome variables proved to be a very responsive index, especially at the $20 \%$ threshold. Directly asking for change can also reduce scatter, even though the answer may be biased towards the current condition. Evidenced for this is shown by the high responsiveness of the patient change question and the MACTAR (that incorporates many change items). The responsiveness of functional scales may be partly explained by the fact that they generally comprise several items in a multi-item questionnaire. Nevertheless, a set of two physical function questions on a six point Likert scale was also responsive.

The strong influence of format and content of the patient's global assessment questions on responsiveness is worrying. Similarly, the responsiveness of pain as a measure depends on the format. It is likely that the focus of doctors (or other assessors) is on the patients' disease, but this seems not always be the case for the patients themselves. Although not specified in great detail in the original formulation of the WHO/ILAR core set, we advocate focusing the format of patient oriented instruments on the disease, and paying close attention to the exact wording of the question(s).

Utility scores are advocated as a generic measure of therapy benefits. The two methods to derive utilities proved to have quite different levels of responsiveness. The rating scale (which is a patient preference rather than a true utility) performed adequately (comparable to observer's global assessment on VAS). However, the standard gamble method (a true utility because choices are made in a situation of uncertainty) showed low responsiveness. Economists prefer standard gamble because it conforms better to theoretical principles, but in practice its application was hindered by limited comprehension of the method by our patients and their risk aversive attitude. This phenomenon has been seen before in patient groups with a non-fatal or chronic disease $[31,32]$. 
The data on between-group discrimination must be interpreted with caution. The extent of differences between the groups was not known before the trial, and may have been large in comparison with expected differences in current and future head-to-head trials. Nevertheless, the results are unique and extremely interesting as they indicate that responsiveness, the ability to detect change, may not parallel the capacity to discriminate between different levels of response. Both the ACR improvement criteria (at various percentage levels) and the EULAR response criterion showed excellent discriminatory capacity. This is at odds with the other trials in the review of Felson et al [32], who concluded that $20 \%$ remained the best cut off point for the ACR criteria. A possible explanation is the relatively large contrast between treatment groups in the COBRA study [34]. Other indices were also better discriminators than most single measures, with the exception of grip strength. In contrast, the discriminatory capacity of the MACTAR, though good, was less than expected based on its excellent responsiveness. This difference in performance between the HAQ and the MACTAR is hard to explain, and will need replication in other studies. Grip strength was included in the design of the trial based on the work of Anderson et al [22]. Despite its good performance in trials up to 1989 , it was eventually excluded in the core set for reasons of redundancy. Nevertheless, in early RA the fact that it is a composite measure of hand function with pain, swelling, stiffness, and muscle strength may contribute to its excellent performance. Muscle strength, particularly, may be a physical function variable with potency in early and established RA [35].

From published reports we know that different responsiveness statistics - also those that are solely based on change from therapeutic intervention - may [36] or may not [37] yield different rank orders. In general, rankings based on paired Student's $t$ test values and SRM will only be discrepant when different sample sizes are used for different measures. SRM is least influenced by sample size as it avoids the use of standard error of the mean in the denominator. Sample size was not an issue in this report as few values were missing. ES and SRM generally yield similar ranks, though discrepancies occur when the within-group $\mathrm{SD}$ at baseline (the denominator in the ES calculation) differs much from the SD of within-group change (the denominator in the SRM calculation). Obviously, ES cannot be calculated for measures that directly evaluate change, because their do not have a baseline variance. It may be a typical feature of these transitional measures - and indices that include change questions, such as the MACTAR - to pair a large SRM with a relatively small unpaired $t$ value.

Despite their strong evidence, the data represent only one study in one subgroup - that is, early RA. The generalisability of our findings may be slightly limited, as the effects of treatment in the combined-treatment arm were large compared with many other trials in RA, but similar to those seen in recent anti-TNF trials. A meta-analysis of effectiveness of low-dose corticosteroids in RA reported somewhat smaller ES in measurements of grip strength, swollen and joint tender count, and (0.4-1.0) than we did; in particular, the ES values found in the corticosteroid treatment arms were smaller [38]. However, the effect in the sulphasalazine group resembles that found in trials of methotrexate and intramuscular gold [39-42]. Thus for studies of such moderately effective drugs, the ranking of the sulphasalazine group might be more appropriate. 
Analyses on floor and ceiling effects showed that ESR and tender and swollen joint count were vulnerable to a certain degree of floor effect. The study's inclusion criteria towards disease duration and disease activity, with evaluation based on ESR and joint counts, probably prevented a serious floor effect in the study group. With global and pain assessments on a visual analogue scale, people tend to put their mark somewhere at the middle of the scale.

Buchbinder et al [43] studied the ability of end points to discriminate between treatment effects in a placebo controlled trial of cyclosporin in RA. As the difference between cyclosporin and placebo was the primary study question of that trial, their approach is similar to the post hoc discrimination tests between treatment groups in this report. Compared with the COBRA study, differences between treatment groups were smaller for and swollen joint counts but similar in other measures. They found physician's and patient's global measures (measured as a change question), as well as the AIMS pain subscale to be most discriminatory, and and pain (five point scale) to be least discriminatory, with all other core set measures, including another physician's global question, the HAQ and a modification of the MACTAR (that is, PET), falling in between. These results agree with our observation on the importance of the exact format of the questions. The discrepancy found in the is expected: lack of responsiveness of is well known during treatment with cyclosporin. More surprising is the relatively poor performance of the physical function questionnaires. It may be that the cyclosporin trial included patients with longstanding disease and more fixed disability that was less likely to respond to treatment. Patients in the COBRA study had a median disease duration of only 4 months.

Differences in responsiveness, and especially discrimination, have important implications for trial design. The use of responsive and discriminative measures allows reduced patient numbers or detection of smaller - yet relevant - differences between groups. This is important especially in trials of early RA. Simpler trial design through use of a limited number of measures saves costs and effort, and facilitates interpretation of the results. In routine patient care, use of a limited number of highly responsive measures facilitates the collection and interpretation of long-term follow-up data. Obviously, additional measures should be applied according to the characteristics of the individual patient.

In summary, this study convincingly shows that responsiveness and the ability to discriminate between different levels of response are not identical concepts. The data provide strong evidence for the responsiveness and discriminatory capacity of the WHO/ILAR core set as well as the ACR and EULAR response criteria in the study of moderately and strongly effective drugs in early RA. However, where information is elicited from the patient, researchers should select and focus their instruments on the disease, as performance is strongly dependent on the exact format of questions. 


\section{References}

1. Boers M, Tugwell P, Felson DT, et al. World Health Organisation and International League of Associations for Rheumatology core endpoints for symptom modifying antirheumatic drugs in rheumatoid arthritis clinical trials. J Rheumatol 1994; 41 suppl:86-9.

2. Felson DT, Anderson J, Boers M, et al. The American College of Rheumatology preliminary core set of disease activity measures for rheumatoid arthritis clinical trials. The Committee on Outcome Measures in Rheumatoid Arthritis Cinical Trials. Arthritis Rheum 1993; 36:729-40.

3. Felson DT, Anderson JJ, Boers M, et al. American College of Rheumatology preliminary definition of improvement in rheumatoid arthritis. Arthritis Rheum 1995; 38:727-35.

4. van Gestel AM, Prevoo MLL, van ' $t$ Hof MA, van Rijswijk MH, van de Putte LBA, van Riel PLCM. Development and validation of the European League Against Rheumatism response criteria for rheumatoid arthritis. Arthritis Rheum 1996; 39:34-40.

5. Boers M, Brooks P, Strand CV, Tugwell P. The OMERACT Filter for outcome measures. J Rheumatol 1998; 25:198-9.

6. Liang MH. Evaluating measurement responsiveness. J Rheumatol 1995; 22:1191-2.

7. Norman GR, Stratford P, Regehr G. Methodological problems in the retrospective computation of responsiveness to change: The lesson of Cronbach. J Cin Epidemiol 1997; 50:869-79.

8. Pincus T, Stein OM. What is the best source of useful data on the treatment of rheumatoid arthritis: clinical trials, clinical observations, or clinical protocols? J Rheumatol 1995; 22:1611-7.

9. Boers M, Verhoeven AC, Markusse HM, et al. Randomised comparison of combined step-down prednisolone, methotrexate and sulphasalazine with sulphasalazine alone in early rheumatoid arthritis. Lancet 1997; 350:309-18.

10. Arnett FC, Edworthy SM, Bloch DA, et al. The American Rheumatism Association revised criteria for the classification of rheumatoid arthritis. Arthritis Rheum 1988; 31:315-24.

11. The cooperating clinics committee of the American Rheumatism Association. A seven-day variability study of 499 patients with peripheral rheumatoid arthritis. Arthritis Rheum 1965; 8:302-34.

12. Fries JF, Spitz PW, Young DY. The dimensions of health outcome: the Health Assessment Questionnaire, disability and pain scales. J Rheumatol 1982; 9:789-93.

13. Siegert CEH, Vleming LJ, Vanderbroucke JP, Cats A. Measurement of disability in Dutch rheumatoid arthritis patients. Cin Rheumatol 1984; 3:305-9.

14. Jones E, Hanly JG, Mooney R, et al. Strength and function in the normal and rheumatoid hand. J Rheumatol 1991; 18:1313-8.

15. Beaton DE, Hogg-Johnson S, Bombardier C. Evaluating changes in health status: reliability and responsiveness of five generic health status measures in workers with musculoskeletal disorders. J Cin Epidemiol 1997:

16. Tugwell P, Bombardier C, Buchanan WW, Goldsmith C, Grace E, Hanna B. The MACTAR patient preference disability questionnaire: an individualized functional prionity approach for assessing improvement in physical disability in clinical trials in rheumatoid arthritis. J Rheumatol 1987; 14:446-51.

17. Fitzpatrick R, Ziebland S, Jenkinson C, Mowat A. A comparison of the sensitivity to change of several health status instruments in rheumatoid arthritis. J Rheumatol 1993; 20:429-36.

18. Verhoeven AC, Bibo JC, Boers M, Engel GL, van der Linden S. Cost- effectiveness and cost-utility of combination therapy in early rheumatoid arthritis: randomized comparison of combined step-down prednisolone, methotrexate, and sulphasalazine with sulphasalazine alone. Br J Rheumatol 1998; 37:1102-9.

19. Bennett K, Torrance GR, Tugwell P: Methodological challenges in the development of utility measure of health-related quality of life in rheumatoid arthritis. Control Cin Trials 1991; 12 suppl:118-28. 
20. Bakker CH, Rutten-van Mölken MPMH, van Doorslaer EKA, Bennet $\mathrm{K}$, van der Linden S. Health related urility assessment by rating scale and standard gamble in patients with ankylosing spondylitis or fibromyalgia. Patient Educ Counsel 1993; 20:145-52.

21. Smythe HA, Helewa A, Goldsmith CH. 'Independent assessor' and 'pooled index' as techniques for measuring treatment effects in rheumatoid arthritis. J Rheurnatol 1977; 4:144-52.

22. Anderson JJ, Felson DT, Meenan RF, Williams HJ. Which traditional measures should be used in rheumatoid arthritis clinical trials? Arthritis Rheum 1989; 32:1093-9.

23. Van der Heijde DMFM, van ' $t$ Hof MA, van Riel PLCM, et al. Judging disease activity in clinical practice in rheumatoid arthritis: first step in the development of a disease activity score. Ann Rheum Dis 1990; 49:916-20.

24. Pinals RS, Masi AT, Larsen RA. Preliminary criteria for clinical remission in rheumatoid arthritis. Arthritis Rheum 1981; 24:1308-15.

25. Prevoo MLL, van Gestel AM, van 't Hof MA, van Rijswijk MH, van de Putte LBA, van Riel PLQM. Remission in a prospective study of patients with rheumatoid arthritis. American Rheumatism Association preliminary remission criteria in relation to the Disease Activity Score. Br J Rheumatol 1996; 35:1101-5.

26. Tugwell $\mathrm{P}$, Pincus $\mathrm{T}$, Yocum $\mathrm{D}$, et al. Combination therapy with cyclosporine and methotrexate in severe rheumatoid arthritis. NEngl J Med 1995; 333:137-41.

27. Liang MH, Fossel AH, Larson MG. Comparisons of five health status instruments for orthopedic evaluation. Med Care 1990; 28:632-42.

28. Kazis L, Anderson J, Meenan R. Effect sizes for interpreting changes in health status. Med Care 1989; 27:S178-9.

29. Beaton BE, Hogg-Johnson S, Bombardier C Evaluating changes in health status: Reliability and responsiveness of five generic health status measures in workers with musculoskeletal disorders. J Cin Epidemiology 1997; 50:79-93.

30. Streiner DL, Norman GR. Health measurement scales. A practical guide to their development and use. 2nd ed. Oxford: Oxford University Press, 1995; 164-6.

31. Bakker CH, Rutten-van Mölken MPMH, van Doorslaer EKA, Bennet $\mathrm{K}$, van der Linden S. Feasibility of utility assessment by rating scale and standard gamble in patients with ankylosing spondylitis or fibromyalgia. J Rheumatol 1994;21:269-74.

32. Goossens MEJB, Rutten-van Mölken MPMH, Leidl RMJ, Bos SGPM, Vlaeyen JWS, Teeken-Gruben NJG. Cognitive-educational treatment of fibromyalgia: Randomized clinical trial. II Economic evaluation. J Rheumatol 1996; 23:1246-54.

33. Felson DT, Anderson JJ, Lange MLM, Wells G, LaValley MP. Should improvement in rheumatoid arthritis clinical trials be defined as fifty percent or seventy percent improvement in core set measures, rather than twenty percent? Arthritis Rheum 1998; 41:1564-70.

34. Anderson JJA, Wells G, Verhoeven AC, Felson DT. Factors predicting response to treatment in rheumatoid arthritis: the importance of disease duration. Arthritis Rheum 2000; 43:22-9.

35. Stucki G, Bruhlmann P, Stucki S, Michel BA. Isometric muscle strength is an indicator of self-reported physical functional disability in patients with rheumatoid arthritis. Br J Rheumatol 1998; 37:643-8.

36. Wright JG, Young NL. A comparison of different indices of responsiveness. J Cin Epidemiol 1997: 50:239-46.

37. Stucki G, Liang MH, Fossel AH, Katz JN. Relative responsiveness of condition-specific and generic health status measures in degenerative lumbar spine stenosis. J Cin Epidemiol 1995; 48:1369-78.

38. Saag KG, Lindsey AC, Sems K, Nettleman MD, Kolluri S. Low-dose corticosteroids in rheumatoid arthritis. A meta-analysis of their moderate-term effectiveness. Arthritis Rheum 1996; 39:1818-25.

39. Weinblatt ME, Kaplan H, Germain BF, et al. Low-dose methotrexate compared with auranofin in adult rheumatoid arthritis; a thirty-six-week, double-blind trial. Arthritis Rheum 1990; 33:330-8.

40. Ward JR, Williams HJ, Egger MJ, et al. Comparison of auranofin, gold sodium thiomalate, and placebo in the treatment of rheumatoid arthritis; a controlled clinical trial. Arthritis Rheum 1983; 26:1303-15. 
41. Rau R, Herborn G, Karger T, Menninger H, Elhardt D, Schmitt J. A double blind randomized parallel trial of intramuscular methotrexate and gold sodium thiomalate in early erosive rheumatoid arthritis. J Rheumatol 1991; 18:328-33.

42. Jeurissen MEC, Boerbooms AMT, van de Putte LBA, et al. Methotrexate versus azathioprine in the treatment of rheumatoid arthritis. A forty-eight-week randomized double-blind parallel trial. Arthritis Rheum 1991; 34:961-72.

43. Buchbinder R, Bombardier C, Yeung M, Tugwell $P$. Which outcome measures should be used in rheumatoid arthritis clinical trials? Cinical and quality-of-life measures' responsiveness to treatment in a randomized controlled trial. Arthritis Rheum 1995; 38:1568-80.

\section{Appendix}

Formats of items with patient assessment:

Assessment of change in disease activity (7 point Likert scale)

Q. When you think of your arthritis during the two weeks before the first interview, how much better or worse overall has your arthritis become? $\quad \square_{7}$ a great deal better

$\square_{6}$ moderately better

$\square_{5}$ slightly better

$\square_{4}$ no change

$\square_{3}$ slightly worse

$\square_{2}$ moderately worse

$\square_{1}$ a great deal worse

Assessment of disease activity (5 point Likert scale)

Q. When you think of your arthritis,

how would you say your condition has been over the past week ? ${ }_{5}$ good

$\begin{array}{ll}\square_{4} & \text { reasonably good } \\ a_{3} & \text { moderate } \\ \square_{2} & \text { poor } \\ \square_{1} & \text { very poor }\end{array}$

Assessment of physical function (two questions, 6 point Likert scale)

Qa. How would you say your overall physical functioning has been ?

Over the last week you think of it as ...

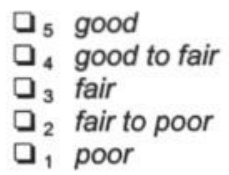

Qb. Is your physical function not as good as it might be because of your arthritis?

$a_{0}$ yes $a_{1}$ no

Global assessment (10 cm visual analogue scale)

Q. How has your general health been during the past week?

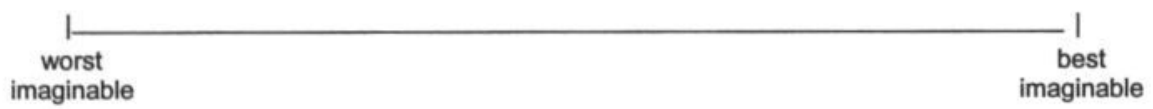

Pain assessment (10 cm visual analogue scale)

Q. How much pain did you have during the past week ?

no pain
at all $\quad \begin{gathered}\text { worst pain } \\ \text { imaginable }\end{gathered}$




\section{CHAPTER 10}

\section{AMERICAN COLLEGE OF}

RHEUMATOLOGY CRITERIA FOR

IMPROVEMENT IN RHEUMATOID

ARTHRITIS SHOULD ONLY BE

CALCULATED FROM SCORES THAT

DECREASE ON IMPROVEMENT

Maarten Boers, Arco Verhoeven, Sjef van der Linden

Arthritis \& Rheumatism 2001; 44: 1052-5. 


\title{
American College of Rheumatology Criteria For Improvement In Rheumatoid Arthritis Should Only Be Calculated From Scores That Decrease On Improvement
}

\begin{abstract}
Objective: Change in a patient's condition is expressed as a percentage of the baseline value for a core set of measures in the American College of Rheumatology (ACR) improvement criteria for rheumatoid arthritis (RA), and this is used as the basis to decide whether a patient has improved. The result is dependent on whether the underlying measure has a score that increases or decreases on improvement. We examined the importance of this effect in the application of the ACR improvement criteria.

Methods: Data were obtained from the COBRA trial, in which 155 patients with early active RA had been randomised to receive either combination treatment with step-down prednisolone, methotrexate, and sulphasalazine or sulphasalazine alone. Patient and physician global assessments were recoded to reflect decreasing scores on improvement. The effects of this difference in scoring systems were compared among 3 response criteria levels $(20 \%, 50 \%$, and $70 \%)$ that are currently being used to assess improvement in RA clinical trials.

Results: Analyses showed that the effects of a decreasing, versus increasing, score on the designation of improvement cannot be ignored, especially at higher percentages of improvement (e.g., $50 \%, 70 \%$ ).

Conclusion: We recommend that percentage improvement in RA be calculated only on scores that decrease on improvement. When necessary, raw data should be recoded before the ACR improvement criteria are applied.
\end{abstract}




\section{Introduction}

Selection and assessment of outcomes in rheumatology remain of particular interest to clinicians. The OMERACT ("Outcome Measures in Rheumatology") initiative provided a forum for international consensus on a core set of end points to be used in rheumatoid arthritis (RA) clinical trials [1]. Subsequently, improvement criteria were formulated by the American College for Rheumatology (ACR) and the European League Against Rheumatism (EULAR) $[2,3]$. The current ACR improvement criteria for RA are designed to reflect a level of $20 \%$ improvement from baseline in the core set measures, and this is used as the basis to decide whether a patient has improved. We present a hitherto unrecognized set of problems in the interpretation of the criteria, a problem we feel becomes more pertinent now that the onginal percentage $(20 \%)$ is frequently replaced by higher percentages of improvement, such as 50 and 70 .

Two distinct problems can arise in the application and interpretation of the ACR criteria, which comprise, in effect, an index composed of ratio measures. 1) When the denominator (the baseline score) in one or more of the component measures is small, the percentage change exaggerates the absolute change. The impact of the exaggeration is dependent on whether the score decreases or increases on improvement. 2) With measures that increase on improvement, the end of the scale limits the possibilities to improve by a certain percentage.

Furthermore, the combination of several ratios into one index compounds the problem; for example, in one patient, some scores may be (much) lower initially than others, leading to differential weighting of the components. Also, the index can combine measures that decrease on improvement with those that increase on improvement.

In most core set measures (joint counts, pain assessments, and acute-phase reactant levels), scores decrease when the patient improves. However, in three of the core set measures (patient and physician global assessment and functional index), the direction of improvement depends on choices made by the investigator. For example, in a global assessment, the direction of the score is determined by the anchors used on the scale. Several interesting measures that are currently not include in the core set (e.g., the Medical Outcomes Study 36-item short form health survey, and utility measures) have scores that increase on improvement. Furthermore, a non-core measure such as grip strength not only increases on improvement, but also has no set maximum. 


\section{Methods}

We analysed the importance of these considerations in the dataset of the COBRA trial [4]. In this trial, 155 patients with early active RA had been randomised to receive either combination treatment with step-down prednisolone, methotrexate, and sulphasalazine (COBRA) or sulphasalazine alone. Both the patient and physician global assessments were initially scored on a 0-10 point scale that increased on improvement. In the final (published) analysis, these assessments were recoded to decrease in score on improvement. We compared the effects of using an increasing versus a decreasing score for improvement in core set measures, according to the levels of improvement currently being used in RA cliniacal trials: $20 \%, 50 \%$, and $70 \%$.

\section{Results}

The relationship between the initial score and end score (Figures 10.1A and 10.1B) and between the initial score and the change in the initial score (Figures 10.1C and 10.1D) for a measure that increases on improvement (Figures 10.1A and 10.1C) and a measure that decreases on improvement (Figures 10.1B and 10.1D) was compared among the three different improvement criteria levels. In the case of a score that increases on improvement, patients deemed severe (starting with low scores) could easily improve by more than $100 \%$, but patients considered to be mild or moderately severe on initial scores were limited in their percentage improvement by the end of the scale. Thus, a patient reaching the optimum score (in this case, 10) may not be classified as improved.

In contrast, in measures that decrease on improvement (Figures $10.1 \mathrm{~B}$ and D), the relation between the initial score and end score (or the change) remains interpretable at every percentage. The disadvantage of exaggeration at low initial scores (in this case, in patients with mild disease) is still a factor, however, a patient can never improve by more than $100 \%$. At $100 \%$ improvement, the score is always at its optimum (zero). Thus, a patient with an initial score of 1 can reach $20 \%$ improvement through a decrease of 0.2 points, a change that is probably not clinically relevant; $100 \%$ improvement is reached when the final score is zero.

Table 10.1 shows the total number of occurrences of improvement according to the physician's global assessment (summed over the four assessment times; thus, each patient could contribute a maximum of four occurrences of improvement), according to treatment group and percentage improvement among the patients in the COBRA trial. We included in our analysis the occurences of improvement that were either added or removed, as well as the net change, after recoding the global assessements to reflect decreasing scores with increasing improvement, which gave us the final count for comparison. A substantial number of occurrences of improvement was affected by the recoding. Because some of the effects cancelled out, our results show that recoding has a moderate-to-small positive net effect on the number of occurrences of impovement on the basis of the $20 \%$ and $50 \%$ improvement criteria, and a more prominent negative effect on the number of occurences meeting an improvement level of $70 \%$. Moreover, the effects were not completely equal across treatment groups. Recoding the patient global assessments had similar effects (data not shown). 
scores increase on improvement

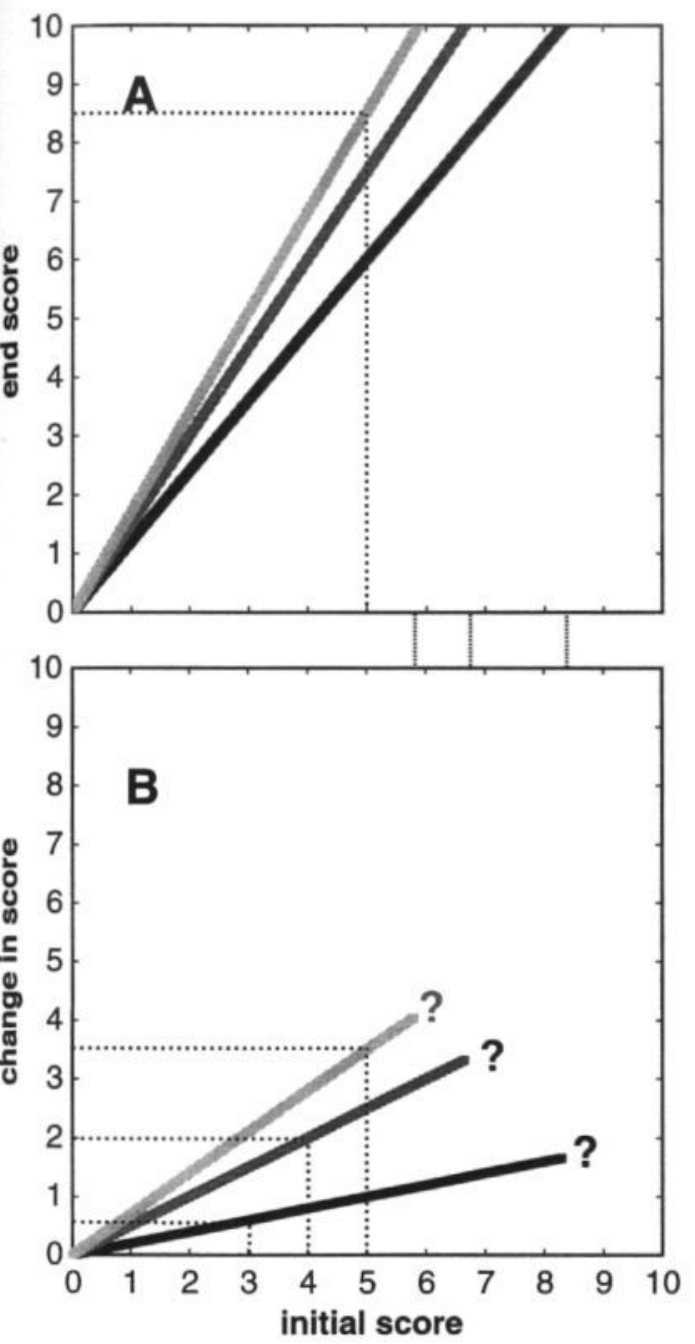

worst score scores decrease on improvement
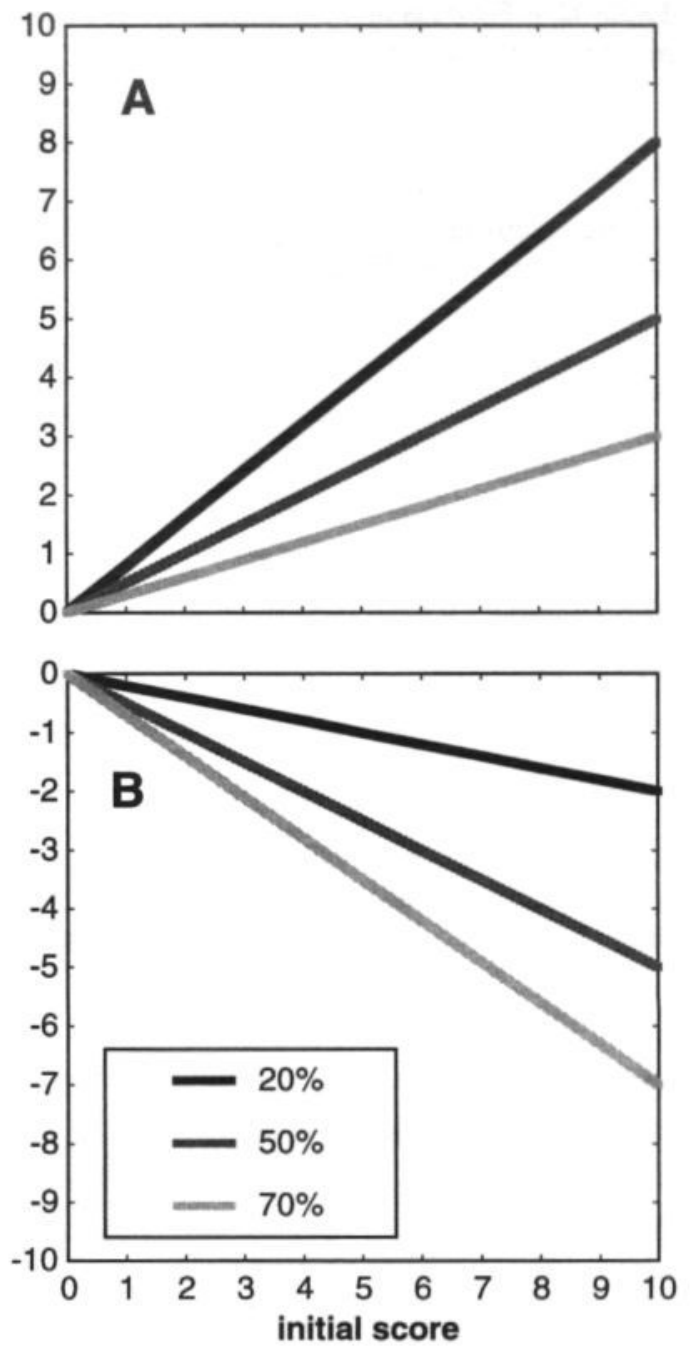

best score

worst score

Figure 10.1: The relationship between the initial score and end score (A and $\mathbf{B})$ and the change in the initial score (C and $\mathrm{D})$ for a measure that increases on improvement $(\mathrm{A}$ and $\mathrm{C})$ and a measure that decreases on improvement (B and D). For example, a patient with an initial score of 5 who improves by $70 \%$ will increase the score by 3.5 (C) to end with a score of 8.5 (A) (see dotted lines; examples for $50 \%$ improvement at an initial score of 4 , and $20 \%$ at an initial score of 3 are also indicated). In the case of an increas in score on improvement, patients cannot improve beyond the maximum score; therefore, lines in $\mathrm{C}$ are truncated at that point. Thus, patients with intermediate or high initial scores (corresponding to intermediate-to-low disease activity) can go into remission (maximum score) without satisfying the improvement criterion. 
Table 10.1: Total occurences of improvement in physician global assessment during the COBRA trial, by ACR criterion level and treatment group*

\begin{tabular}{|c|c|c|c|c|c|c|c|c|c|c|}
\hline \multirow{2}{*}{$\begin{array}{c}\text { ACR } \\
\text { improvement } \\
\text { criteria }\end{array}$} & \multicolumn{5}{|c|}{$\begin{array}{l}\text { Combined treatment } \\
\qquad(n=76)\end{array}$} & \multicolumn{5}{|c|}{$\begin{array}{l}\text { Sulphasalazine } \\
\qquad(n=79)\end{array}$} \\
\hline & Original & + & - & Net (\%) & Final & Original & + & - & Net (\%) & Final \\
\hline $20 \%$ & 217 & 25 & 4 & $21(10)$ & 238 & 178 & 23 & 13 & $10(6)$ & 188 \\
\hline $50 \%$ & 170 & 41 & 31 & $10(6)$ & 180 & 117 & 32 & 26 & $6(5)$ & 123 \\
\hline $70 \%$ & 134 & 34 & 53 & $-19(14)$ & 115 & 91 & 23 & 39 & $-16(18)$ & 75 \\
\hline \multicolumn{11}{|c|}{$\begin{array}{l}\text { * Each patient can contribute up to four occurrences of improvement for each percentage level of } \\
\text { American College of Rheumatology (ACR) improvement. The original count is on scores that increase on } \\
\text { improvement. On recoding scores to reflect a decrease in score on improvement, new occurrences of } \\
\text { improvement were added ( }+ \text { ) and "no longer occurrences of improvement" were subtracted }(-) \text { to obtain } \\
\text { the final count. Net change is shown as an absolute count and as a percentage of the original count. }\end{array}$} \\
\hline
\end{tabular}

Table 10.2 shows the effect of recoding both the physician and the patient global assessments on the total number of patients classified as improved in each treatment group, for two cardinal time points: 28 weeks (the last assessment before prednisolone was tapered and stopped) and 56 weeks (the end of the trial). As previously reported, at 28 weeks the difference between treatment groups was maximal; whereas at 56 weeks it was gone [4]. Again, the net effect of recoding is rather modest, mostly because patients who were not classified as improved on recoding were replaced by others who newly classify as improved. However, at the $70 \%$ improvement criteria level, the effects are more pronounced. 
Table 10.2: Total occurences of improvement in physician global assessment during the COBRA trial, by ACR criterion level and treatment group*

Time point

ACR

improvement criteria
Combined treatment

$$
(n=76)
$$

Sulphasalazine

$(n=79)$

Original +- Net Final

Original +- Net Final

28 weeks

$\begin{array}{rrrrrrrrrrr}20 \% & 68 & 4 & 0 & 4 & 72 & 48 & 1 & 0 & 1 & 49 \\ 50 \% & 47 & 2 & 0 & 2 & 49 & 23 & 4 & 0 & 4 & 27 \\ 70 \% & 29 & 4 & 4 & 0 & 29 & 8 & 5 & 3 & 2 & 10\end{array}$

56 weeks

$\begin{array}{lllllllllll}20 \% & 46 & 3 & 0 & 3 & 49 & 46 & 2 & 0 & 2 & 48 \\ 50 \% & 21 & 5 & 1 & 4 & 25 & 25 & 4 & 0 & 4 & 29 \\ 70 \% & 11 & 1 & 1 & 0 & 11 & 11 & 3 & 3 & 0 & 11\end{array}$

* Each patient was assessed at 28 and 56 weeks for improvement. The original ACR criterion is based on scores of physician and patient global assessment that increased on improvement. On recoding to reflect a decrease in score on improvement, new patients who met the criterion of improvement were added $(+)$ and patients who no longer met the criterion were subtracted $(-)$, to obtain the final percentage.

\section{Discussion}

On the basis of methodological considerations and the above results, but also to facilitate comparisons between trials, we recommend that percentage improvement be calculated only on scores that decrease on improvement. When necessary, raw data should be recoded before the ACR criteria are applied. In practice, the effect of disregarding this advice seems modest for the ACR 20\% improvement criteria, but results may be biased when higher percentages of improvement are used. 


\section{References}

1. Boers M, Tugwell P, Felson DT, et al. World Health Organisation and International League of Associations for Rheumatology core endpoints for symptom modifying antirheumatic drugs in rheumatoid arthritis clinical trials. J Rheumatol Suppl 1994; 41:86-9.

2. Felson DT, Anderson J, Boers M, et al. American College of Rheumatology preliminary definition of improvement in rheumatoid arthritis. Arthritis Rheum 1995; 38:727-35.

3. van Gestel AM, Prevoo MLL, van ' $t$ Hof MA, van Rijswijk MH, van de Putte LBA, van Riel PLCM. Development and validation of the European League Against Rheumatism response criteria for rheumatoid arthritis. Arthritis Rheum 1996; 39:34-40.

4. Boers M, Verhoeven AC, Markusse HM, et al. Randomised comparison of combined step-down prednisolone, methotrexate and sulphasalazine with sulphasalazine alone in early rheumatoid arthritis. Lancet 1997; 350:309-18. 


\section{CHAPTER 11}

\section{VALIDITY OF THE MACTAR QUESTIONNAIRE AS \\ FUNCTIONAL INDEX \\ IN A RHEUMATOID ARTHRITIS \\ CLINICAL TRIAL}

Arco Verhoeven, Maarten Boers, Sjef van der Linden

Journal of Rheumatology 2000; 27: 2801-9. 


\section{Validity of MACTAR Questionnaire as Functional Index in a Rheumatoid Arthritis Clinical Trial}

\section{Abstract}

Objectives. The McMaster Toronto Arthritis patient preference questionnaire (MACTAR) is a functional index that measures change in impaired activities selected by each patient in a baseline interview, and change in rheumatoid arthritis (RA) disease activity. In addition, it contains questions on the state of physical, social, and emotional function, overall health, and their relation to RA. We evaluated MACTAR's feasibility and validity (content validity, construct validity, and responsiveness).

Methods. A randomised trial of combined treatment in 155 patients with early RA; patients' mean age at baseline was 50 years and median disease duration since diagnosis was 4 months.

Results. Feasibility: MACTAR requires trained interviewers. In the trial, interviews took about 15 minutes. In longer-term follow-up, activities selected at baseline may become less relevant as the pattern of disability changes. Follow-up from 153 patients (99\%) was available. At least 5 impaired activities were identified and ranked by 147 patients (95\%), interviewers could follow $99 \%$ of these. The scoring system proved complex and required amendments. Content validity: Although its main focus is physical function, the MACTAR also contains generic questions; $75 \%$ of the patients named at least one impaired activity from the category "mobility". Only $48 \%$ were covered by Health Assessment Questionnaire (HAQ) items. Construct validity: MACTAR scores correlate highly with other functional indices and with measures of disease activity. Responsizemess: At 16 weeks the standardised response mean for the total MACTAR score in the combinedtreatment group was excellent, at 2.2. Items that directly address change were even more responsive.

Conclusion. The MACTAR interview is a valid and highly responsive instrument to assess change in functional ability of patients with early RA with active disease. It provides insight into problems - mainly of physical function - that really matter to patients. For standard clinical trials and clinical care, feasibility of the MACTAR is limited and the simpler HAQ remains the instrument of choice. 


\section{Introduction}

Physical function, an important outcome in rheumatoid arthritis (RA) is included in the World Health Organization/ International League of Associations for Rheumatology (WHO/ILAR) core set for assessment of this ubiquitous disease [1]. Examples of widely used questionnaires to measure physical function are the Stanford Health Assessment Questionnaire (HAQ) [2] and Arthritis Impact Measurement Scale (AIMS) [3]. However, these measures do not include patient priorities. Including such priorities may lead to better face validity and responsiveness [4]. An example of a functional scale that takes account of patient priorities is the McMaster Toronto Arthritis patient preference disability questionnaire (MACTAR) [5]. Its developers noted good responsiveness in RA patients in a controlled trial that demonstrated clinically important change. In the MACTAR, an interviewer elicits which 5 impaired activities are most important to the individual patient, and follows their changes over time. The MACTAR also includes questions on social and emotional functioning and probes whether any problems are due to arthritis (see Appendix). Because of this format, the MACTAR concept of function may be more comprehensive than that of traditional fixed-item questionnaires such as the HAQ, and reveal issues that really matter to the patient.

The recent COBRA study compared combined treatment of step-down prednisolone, methotrexate, and sulphasalazine with single sulphasalazine treatment in early RA patients with active disease [6]. In the setting of this multicentre randomised trial, we studied feasibility, content validity (comprehensiveness), construct validity (correlation with other measures) and responsiveness (discriminatory validity) of the MACTAR interview.

\section{Patients and Methods}

In the COBRA multicenter randomised controlled trial enrollment of RA patients (ACR criteria) with early, active disease, took place in 10 clinical centers in The Netherlands and Belgium between May 1993 and May 1995. Patients were randomised to combination therapy of diseasemodifying anti-rheumatic drugs with step-down prednisolone, methotrexate and sulphasalazine ( $n$ $=76$ ) or single therapy with sulphasalazine $(n=79)$. Methotrexate and sulphasalazine doses were fixed at $7.5 \mathrm{mg}$ per week and $2000 \mathrm{mg}$ per day, respectively, but daily prednisolone doses were 60 $\mathrm{mg}$ in week $1,40 \mathrm{mg}$ in week $2,25 \mathrm{mg}$ in week $3,20 \mathrm{mg}$ in week $4,15 \mathrm{mg}$ in week $5,10 \mathrm{mg}$ in week 6 , and $7.5 \mathrm{mg}$ from week 7 onwards. At baseline patients' mean age was 50 years and median disease duration (since ACR diagnosis [7D 4 months. Apart from the MACTAR questionnaire several other measures of function and disease activity were assessed at baseline, and in weeks 16, 28, 40 and 56. This included validated Dutch versions of the HAQ [8] and AIMS [9-10], and grip strength assessed by a vigorimeter [11].

A priori, the largest improvement was expected at the first major assessment (after 16 weeks) in the combined-treatment group, and a smaller - but also clinically relevant improvement - in the sulphasalazine group. 


\section{The MACTAR instrument}

The term 'semi-structured interview' rather than questionnaire, best describes the MACTAR because interviewers fill out the answers on standard forms. The MACTAR comprises two parts (see Appendix for a 'back translation' of the Dutch version we used). The first part - which we refer to as 'the transitional questions' - starts with a question about patient perceived change in arthritis activity (7 point Likert scale; question 1d). It is complemented with questions aimed at the change in ability to perform 5 impaired activities, elicited and priority ranked by the patient during a baseline interview (question 2). Patients are asked to consider daily routine problems they face as a result of their disease. Once they finish identifying problems spontaneously, the interviewer reads a series of 'probes' to assist the patient. These probes are open-ended questions covering broad areas of function: domestic care, self-care, professional activities, leisure activities, sexuality, social interaction and roles. Patients are allowed to identify up to 10 problems. Subsequently, they are asked to identify and rank the 5 most important problems, i.e. the activities they most eagerly wish to perform without pain or discomfort. The second part of the interview - which we refer to as 'the status questions' - elicits the patient's health status by one question each on general health (question 1a), satisfaction with life (3 point Likert scales; question 3a), psychological, social and emotional well being ( 5 point Likert scales; questions $4 a$, $5 a$ and $6 a$ respectizely). When the questions reveal a less than optimal status, a second question probes whether this is due to arthritis (questions $3 b, 4 b, 5 b$, and $6 b$ ). Presumably, the appreciation of any adverse event due to treatment of the disease is implicitly reflected in these status questions.

Higher scores on the MACTAR reflect improvement. Scores vary from 11 to 47. In its original format, the baseline interview has no scores for the transitional question part. To allow easier interpretation of change between baseline and follow-up we added mock (or dummy) scores (14 points) consistent with a 'no change situation', to baseline total scores. With this adjustment, baseline scores vary from 19 to 39 . Thus, theoretically a change score can vary from -28 (maximum deterioration, with a baseline score of 39 subtracted from a follow-up score of 11) to +28 (maximum improvement, with a baseline score of 19 subtracted from a follow-up score of 47). Also in instances with no available follow-up score, a mock score indicating 'no change' was assigned.

The MACTAR offers the possibility of weighting the impaired activities (in question 2) by an assigned priority rank. The developers suggest weighting the change score in the activity with the highest priority 5 times, the second highest 4 times, et cetera. To evaluate visual analogue scales compared to Likert scales, an extension to the original format of the interview was made by having patients mark perceived change on a 10-centimeter visual analogue scale after they answered the 3-point Likert scale item (question 1a; improved/ no change/ worsened).

The MACTAR interview was translated into Dutch by one of the authors (AV). Next, a rheumatologist $(\mathrm{MB})$ and 2 health scientists compared the translation with the original to verify cross-cultural equivalence. The first version was pre-tested in a selection of volunteers with arthritis. 


\section{Validity issues}

Blinding of the patient and interviewer to treatment allocation is an important validity issue in randomised controlled trials, especially when subjective impressions of patients and assessors are reflected in outcomes. Obviously, the MACTAR scoring reflects such subjective impressions. Optimum blinding was guaranteed by the study design in which assessors did not contact patients between measurements at baseline, and follow-up at week $16,28,40$, and 56 . This was especially pertinent during the first 6 weeks of the protocol, when potential (side) effects of high-dosed prednisolone would be most apparent. Also, the assessors were asked not to discuss disease activity or the blinded treatment effects with the patients

Listing the requirements and time needed for the interviewing assessed feasibility. Also the response rate and loss to follow-up was noted. A second 'baseline interview' was taken at week 56 to repeat the elicitation of impaired activities; the purpose was to evaluate the feasibility of the MACTAR interview in extended follow-up of patients with treated and therefore less active disease. Besides feasibility, aspects of validity that have been studied are content validity (comprehensiveness), construct validity, and responsiveness.

The content validity (comprehensiveness) of the MACTAR was appraised by listing the impaired activities elicited by the patients and matching these with items from the HAQ. Two assessors did this because of the qualitative nature of it. Cohen's $\mathrm{K}$ (Kappa) reflects inter-rater reliability.

Construct validity was assessed by correlating MACTAR scores with other functional indices: we used Pearson's moment correlation with HAQ, AIMS, and grip strength at week 16 of follow-up. As all these indices have the construct 'functional ability' in common, correlations are expected to be high, and specifically higher than correlations between MACTAR and measures of other dimensions of the burden of disease. Tender and swollen joint count (68 and 48 joints [12], respectively), erythrocyte sedimentation rate (ESR), patient's and assessor's global assessments (5 point Likert scale and $10 \mathrm{~cm}$ visual analogue scale, respectively), and patient's pain assessment (10 $\mathrm{cm}$ visual analogue scale), served as measures of such dimensions.

Within-group responsiveness of separate items and parts of the MACTAR was evaluated by standardresponse means (SRMs) [13]. This is the mean observed change within one treatment group divided by the standard deviation of this change. In each treatment group, SRMs at week 16 of MACTAR and other functional, as well as non-functional outcome measures were compared. Confidence intervals of SRMs were calculated under the assumption that its distribution is approximately Gaussian with mean zero and SD of one over the square root of the sample size [14]. To get an impression of between-group discriminative power we also report $t$ values for between-treatment group comparisons at week 16 of follow-up. However, these unpaired $t$ values should be interpreted with caution, as between-group comparison constituted the primary study question. They are less suited for a direct evaluation of outcomes' comparative discriminatory capacity [15]. 


\section{Results}

The main findings of the COBRA trial have been published [6]. Briefly, combined treatment caused almost immediate and strong improvement in disease activity, resulting in similarly strong and significant functional improvement assessed at week 16. Single treatment with sulphasalazine caused lesser but also significant improvement at week 16. Both treatment groups improved further up to week 28. Most of the clinical difference between the groups disappeared when prednisolone was subsequently withdrawn, with no further change after withdrawal of methotrexate. At week 28, the combination therapy group had significantly less progression of radiographic joint damage compared to the sulphasalazine group $(P<0.0001)$, this difference persisted up at least to week 80 , i.e., 1 year after withdrawal of prednisolone.

During the trial, baseline and follow-up MACTAR interviews were taken from 153 patients ( $99 \%$ follow-up). The duration of the interviews at baseline and follow-up was about 15 minutes. No interviews were broken off. The mean number of impaired activities identified by a single patient at baseline was 6.6 (SD: 2.2); 147 patients (95\%) were at that time able to identify and rank at least 5 impaired activities (one patient identified 2, and 7 patients identified 4 activities). Of the total of 765 activities, the interviewers were able to follow $754(99 \%)$ in the follow-up interview at week 16; at week 28, 40 and 56, these numbers were 743 (97\%), 706 (92\%) and 715 (93\%), respectively. In the repeated baseline interview at week 56, data from 83 patients were available. At that moment, with reduced disease activity compared to baseline in the majority of patients, only 55 patients $(66 \%)$ were able to name 5 or more impaired activities. The number of elicited activities per patient at week 56 was not influenced by the (previous) treatment allocation (unpaired $t$ test: $P=0.97$ ). Of the total number of 392 activities named at week 56,339 had a priority rank of 1 to $5 ; 124$ (37\%) were equal or very similar to 'top-five' priority activities named by the same patient at baseline.

The majority of impaired activities ranked as one of 5 most important were in the category 'mobility' with paraphrases referring to bicycling (peddle, brake, get on and off the bicycle), driving (steer, use gear, park, go in and out the car, open doors), walking, climbing stairs, getting up, ability to be in the same position for long, to kneel down, bend over, reach down or get out of bed; these activities were named 171 of 763 times (22\%). Hundred-and-seventeen patients $(75 \%)$ mentioned at least one 'top-five' activity that was assigned to the category "mobility". Other categories are listed in Table 11.1. The comprehensiveness of the MACTAR is also illustrated by the incomplete coverage of patient elicited items by the HAQ. Both assessors rated that $48 \%$ of the phrases in patient elicited activities were also covered by items from the HAQ (inter-rater agreement was good: Cohen's $\mathrm{K}=0.81$ ).

High correlations were found between total MACTAR score, its partitions and other functional indices, in descending order: HAQ, AIMS mobility, and grip strength (all $P<0.0001$, Table 11.2). The correlation between scores on the item with which the patients were asked to rate change in disease activity (question 1d) and change scores in five high-priority activities (questions 2a-e) together forming the transitional part of the MACTAR - was 0.72 at week 16 . At week 16, 
correlation between total MACTAR and 'non-functional' outcomes (i.e., ESR, various pain scales, joint counts, and global assessments) was also high, ranging from 0.38 to 0.77 (all $P<0.0001$ ).

Table 11.1: Activities elicited by the MACTAR interview, that the patients wanted to improve most eagerly (max. 5 activities per patient, 2 treatment groups with 155 patients, total of 765 activities elicited).

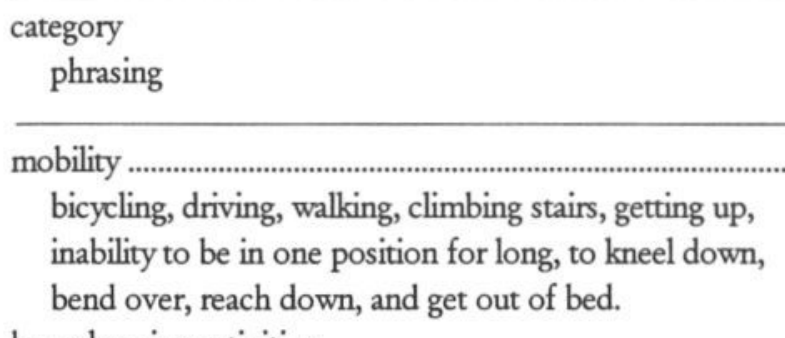

housekeeping activities

vacuum-cleaning, cooking with heavy pans, mopping,

washing windows or dishes, shopping, peeling potatoes,

doing small repairs, tying up garbage bags, and lifting buckets.

self care activities

$123(16 \%)$

$94(61 \%)$

washing body and hair, dressing (especially socks,

zippers, bra and sweater), combing, shaving, drying hair,

wiping, get off the toilet, or get out the bathtub.

leisure activities

$111(15 \%)$

$87(56 \%)$

dancing, sports including jogging, tennis, aerobics, billiards

and swimming, gardening, knitting, making music, woodcarving,

bird raising, painting, going out to theater or cinema.

profession

$37 \quad(5 \%)$

$33(21 \%)$

eating and drinking

$34(4 \%)$

$30(19 \%)$

cutting meat or bread, open milk pack or bottle, pour from bottle.

family role (in relation to spouse, partner or parents)

child care

miscellaneous, upper extremities

$23(3 \%)$

shaking hands, turning keys, writing, leaning on wrists,

lifting unspecified items, using pair of scissors, reaching up.

social roles

$22(3 \%)$

$22(14 \%)$

sexuality

$18 \quad(2 \%)$

$18(12 \%)$

$8 \quad(1 \%)$

$7 \quad(5 \%)$

not activities but complaints like stiffness and fatigue.

sleeping and resting.

$6(1 \%)$ 
Compared to other outcome measures, the total MACTAR interview showed high responsiveness as reflected by SRMs (Table 11.3). The MACTAR with weighted scores had slightly better responsiveness. Questions that ask directly for change (so-called transitional questions) showed best responsiveness. The responsiveness of 5 summarised $10 \mathrm{~cm}$ visual analogue scales elucidating changes in impaired activities, was not better than the corresponding summarised scores from the original questions with 3 response categories (data not shown). In this trial with large between-group differences at week 16 , all function measures were capable to discriminate significantly between the treatment groups. HAQ, grip strength, and MACTAR (especially the part with transitional questions) served best for this matter.

Table 11.2: Correlations between outcomes at week 16 follow-up ( $n=153$ ).

$\begin{array}{cccc}\text { total } & \text { status } & \text { transitional } & \text { transitional } \\ \text { MACTAR } & \text { questions } & \text { questions } & \text { questions } \\ \text { score } & \text { part * } & \text { part } \dagger & \text { part weighted }\end{array}$

parts of the MACTAR

status questions

transitional questions

transitional questions, weighted

functional outcomes

HAQ

AIMS mobility scale

grip strength

other outcomes

global assessment assessor VAS

global assessment patient 5-point Likert

ArMs pain scale

pain VAS

tender joint count

AIMS self efficacy scale

erythrocyte sedimentation rate

swollen joint count

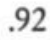

.84

.82

$-.73$

.61

.46

$-.77$

$-.74$

$-.71$

$-.68$

$-.62$

.55

$-.39$

$-.38$

92

8

56

.54

$-.62$

.55

.38

$-.67$

$-.67$

$-.59$

$-.57$

$-.61$

$-.59$

$-.60$

$-.59$

$-.51$

$-.51$

.52

.51

.47

$-.29$

$-.42$

$-.37$

$-.33$

$-.32$

Pearson's correlations (without correction for multiple comparisons); $\mathrm{r} \geq 0.29$ have $P<0.0003$.

*; Status questions have numbers $1 \mathrm{a}, 3 \mathrm{a}, 3 \mathrm{~b}, 4 \mathrm{a}, 4 \mathrm{~b}, 5 \mathrm{a}, 5 \mathrm{~b}, 6 \mathrm{a}$, and $6 \mathrm{~b}$, in baseline and follow-up interviews (see Appendix).

$\dagger$; Transitional questions have numbers $1 \mathrm{~d}$ and $2 \mathrm{a}-\mathrm{e}$, and are not included in the baseline interview. 
Table 11.3: Responsiveness and between-group discrimination of MACTAR and a selection of other outcome measures at week 16. Upper part: comparison MACTAR interview items and total scores. Lower part: other functional and non-functional core set measures.

\begin{tabular}{|c|c|c|c|c|c|c|c|c|c|}
\hline & \multirow[b]{2}{*}{$\begin{array}{l}\text { question } \\
\text { number* }\end{array}$} & \multirow[b]{2}{*}{$\begin{array}{l}\text { range } \\
\text { of scale }\end{array}$} & \multicolumn{3}{|c|}{$\begin{array}{c}\text { combined } \\
\text { treatment } \\
(n=75)\end{array}$} & \multicolumn{3}{|c|}{$\begin{array}{c}\text { sulphasalazine } \\
\text { treatment } \\
(n=78)\end{array}$} & \multirow[b]{2}{*}{$\begin{array}{c}t \\
\text { value }\end{array}$} \\
\hline & & & $\begin{array}{l}\text { mean } \\
\text { changes }\end{array}$ & $\mathrm{SD}_{\Delta} \mathrm{s}$ & SRM & $\begin{array}{c}\text { mean } \\
\text { change }\end{array}$ & $\mathrm{SD}_{\Delta}$ & SRM & \\
\hline \multicolumn{2}{|l|}{ MACTAR status questions } & $5-25$ & 5.5 & 2.7 & 1.2 & 4.2 & 4.7 & 0.9 & 1.8 \\
\hline general health status & $1 \mathrm{a}$ & $1-3$ & 0.8 & 0.7 & 1.1 & .6 & 0.7 & 0.8 & 2.2 \\
\hline satisfaction with life & $3 a b$ & $1-4$ & 0.8 & 1.1 & 0.7 & .6 & 1.0 & 0.6 & 1.0 \\
\hline physical well-being & $4 \mathrm{ab}$ & $1-6$ & 1.8 & 1.4 & 1.3 & 1.3 & 1.5 & 0.9 & 2.2 \\
\hline social well-being & $5 \mathrm{ab}$ & $1-6$ & 0.8 & 1.5 & 0.6 & .8 & 1.7 & 0.5 & 0.2 \\
\hline emotional well-being & $6 \mathrm{ab}$ & $1-6$ & 1.2 & 1.3 & 0.9 & .9 & 1.7 & 0.5 & 1.4 \\
\hline \multicolumn{2}{|l|}{ MACTAR transitional questions } & $6-22$ & 6.6 & 1.9 & 3.5 & 4.3 & 3.5 & 1.2 & 5.4 \\
\hline change in disease activity & $1 \mathrm{~d}$ & $1-7$ & 2.6 & 0.7 & 3.9 & 1.7 & 1.4 & 1.2 & 5.3 \\
\hline change in function & 2 a-e & $5-15$ & 4.0 & 1.4 & 2.8 & 2.6 & 2.3 & 1.1 & 4.5 \\
\hline - with weighting & 2 a-e & $15 \cdot 45$ & 12.0 & 4.2 & 2.9 & 7.8 & 7.0 & 1.1 & 5.0 \\
\hline \multicolumn{2}{|l|}{ MACTAR total score } & $11-47$ & 12.2 & 5.4 & 2.2 & 8.5 & 6.9 & 1.2 & 3.7 \\
\hline - with weighting & & $21-77$ & 20.3 & 7.4 & 2.8 & 13.8 & 11.0 & 1.3 & 4.3 \\
\hline \multicolumn{10}{|l|}{ other functional outcomes } \\
\hline \multicolumn{2}{|l|}{ HAQ } & $0-3$ & -1.1 & 0.7 & 1.5 & -0.4 & 0.6 & 0.8 & 6.2 \\
\hline \multicolumn{2}{|l|}{ grip strength $(\mathrm{kPa})$} & $0-120$ & 22 & 17 & 1.3 & 8 & 13 & 0.6 & 5.8 \\
\hline \multicolumn{2}{|l|}{ AIMS mobility scale } & $7-28$ & 5.3 & 5.8 & 0.9 & 2.1 & 4.5 & 0.5 & 3.9 \\
\hline \multicolumn{10}{|l|}{ core-set outcomes } \\
\hline \multicolumn{2}{|l|}{ pain VAS } & $0-10$ & -3.3 & 2.2 & 1.5 & -1.8 & 2.6 & 0.7 & 4.0 \\
\hline \multicolumn{2}{|l|}{ global assessment patient } & 0.4 & -1.7 & 1.1 & 1.5 & -1.1 & 1.3 & 0.9 & 2.7 \\
\hline \multicolumn{2}{|l|}{$\operatorname{ESR}(\mathrm{mm} / \mathrm{h})$} & $0-150$ & -41 & 29 & 1.4 & -23 & 25 & 0.9 & 4.1 \\
\hline \multicolumn{2}{|c|}{ swollen joint count } & $0-48$ & -9.1 & 6.7 & 1.4 & -4.0 & 6.7 & 0.7 & 4.2 \\
\hline \multicolumn{2}{|c|}{ global assessment assessor VAS } & $0-10$ & -3.0 & 2.3 & 1.3 & -1.4 & 2.3 & 0.6 & 4.3 \\
\hline \multicolumn{2}{|c|}{ tender joint count } & 0.68 & -14 & 12 & 1.2 & -6 & 13 & 0.5 & 4.1 \\
\hline
\end{tabular}

$\mathrm{SD}_{\Delta}$ : standard deviation of changes. SRM: Standardised Response Mean: average change divided by $\mathrm{SD}_{\Delta}$. MACTAR: McMaster Toronto Arthritis questionnaire, VAS: visual analogue scale, HAQ: health assessment questionnaire, AIMS: arthritis impact measurement scale, ESR: erythrocyte sedimentation rate. "; see Appendix. 


\section{Discussion}

This study confirms that the MACTAR interview is a useful extension to the arsenal of functional indices. Over a one-year period, it was valid and highly responsive in patients with early and active RA that started disease-modifying antirheumatic treatment. Changes in disease status and patients' adaptation to their limitations will shift patient priorities and probably make longterm follow-up by MACTAR less useful. This is illustrated by the results of the second baseline interview after one year: 2 thirds of prioritised impaired activities were new, and many patients were unable to name at least 5 impaired activities.

The MACTAR's unconventional method of calculating change and a lack of knowledge on reliability and stability of patient preferences during a stable functional period have been noted as limitations [16]. Interviewing implies extra requirements: interviewers need to be well trained to secure standard implementation. In addition, adequate and accessible administration of baseline records is mandatory. Therefore, is it important that the same interviewer asks questions at baseline as well as follow-up. Specific attention has to be paid to sufficiently circumscribed definition of the elicited and followed impaired activities. Especially with longer follow-up periods, the activities should not be associated with a season. The MACTAR scoring method is somewhat awkward, due to the combination of status and transitional questions. The many different answering formats may cause confusion; also, summarising improvement in scores that increase as patients improve can be bothersome [17]. We recommend the use of baseline mock scores for transitional questions to facilitate comparison between baseline and follow-up scores.

The results of this analysis suggest that the MACTAR's comprehensiveness is indeed better than that of the HAQ, another widely accepted measure; It includes questions on non-physical aspects of function that are not included in the HAQ - but are covered by the AIMS. More importantly, the HAQ only covered half of the items named by patients. The follow-up of impaired functions that are most relevant to the patient is an important part of the interview. Not only do these questions provide insight into patients' priorities that otherwise stay hidden, but - together with the patients' assessment of change in disease activity - they comprise the part of the MACTAR that is exceptionally responsive $(\mathrm{SRM}=3.5)$.

With respect to construct validity, the performances of different parts of the interview were approximately the same. The moderate or high correlation between the total MACTAR score and any of the other functional indices are reassuring (Table 11.2). All these variables seem to reflect one common construct. Presumably this construct is 'functional ability'. The correlation with grip strength is relatively small, but obviously this measure only monitors one aspect of hand function, whereas the MACTAR interview reflects a much broader concept of function. The highest correlations with 'non-function' outcomes are found for global assessments and various painscales. Probably, these are more closely linked to function than process parameters of inflammation such as ESR and swollen joint count. All correlations are significant. This suggests that all reported outcome measures have a construct in common: most likely, this is 'disease activity'. In other words: at least in early RA, functional ability is to a large extent a disease activity measure, rather than a measure of irreversible damage. 
Functional scales are among the most responsive, although other traditional measures also perform adequately. Responsiveness of the MACTAR is large (Table 11.3). The part with transitional questions shows excellent responsiveness in a situation where other measures also indicated significant improvement from baseline in both treatment groups, together with significant differences in improvement between the combined-treatment and sulphasalazine group. The rank order of SRMs is similar in both treatment groups with their different levels of response: high response exemplified by the combined-treatment group, traditional response (similar to that of methotrexate) exemplified by the sulphasalazine group. Both response levels are currently very relevant, as trials with active treatment control are becoming the standard. Consistent ranking of responsiveness at these 2 levels suggests MACTAR will also be responsive at other levels, but we can offer no direct proof of this.

Recording of change with the help of visual analogue scales did not improve responsiveness a great deal, nor did weighting changes in function by assigned priority ranks. Excellent responsiveness of weighted scores for the patient-specific impaired function items was reported by Wright and Young $(\mathrm{SRM}=4.9)$ in patients undergoing elective hip arthroplasty [18]. The COBRA trial also showed a large SRM $=2.9$ for these items (Table 11.3). The slightly better responsiveness of the weighted MACTAR can be explained by the larger contribution to the total score of the more responsive 'transitional questions'. Not surprisingly, the questions that ask directly for change showed the best responsiveness. This type of questioning rules out some of the scatter associated with scoring twice (and calculating change afterwards) [19]. Admittedly, transitional questions may be more prone to socially desirable answering and recall bias but this is not necessarily a problem in randomised trials with adequate blinding of treatment allocation. The range of between-group $t$ values confirms the discriminatory capacity of the MACTAR. However, validation of between group discriminatory capacity requires comparisons with response levels known a priori.

Application of a highly responsive and discriminative functional index is especially useful when it is difficult to include enough patients in a clinical trial during a short period, and when rather small between-group contrasts are to be expected. A priori, the COBRA trial with RA patients with early and active disease, no previous second-line treatment, and active treatment in the control group, was a clear example of such a situation, although the response in the combinedtreatment group clearly exceeded our expectations. In our opinion, when considering the MACTAR there is a strong case to use just the best performing transitional questions part in future trials where high responsiveness of a functional index is a prerequisite to guarantee sufficient statistical power with comparatively small sample size. However, for large trials, for long-term follow-up of existing cohorts, as well as in clinical practice, simpler methods that demand less time and effort, such as the HAQ seem more suited. Indeed, a trade-off has to be made between more concise and more informative but also more laborious measurements to assess function.

In conclusion, the MACTAR is a valid and highly responsive instrument to assess changes in physical function that are relevant to RA patients. Increased relevance comes at a price of reduced feasibility compared to instruments such as the HAQ. 


\section{References}

1. Boers M, Tugwell P, Felson DT, et al. World Health Organization and International League of Associations for Rheumatology core endpoints for symptom modifying antirheumatic drugs in rheumatoid arthritis clinical trials. J Rheumatol 1994; 41 suppl:86-9.

2. Fries JF, Spitz PW, Young DY. Dimensions of health outcome: the Health Assessment Questionnaire. Disability and pain scales. J Rheumatol 1982; 9:727-35.

3. Meenan RF, Gertman PM, Mason JH Measuring health status in arthritis; the Arthritis Impact Measurement Scale. Arthritis Rheum 1980; 23:146-52.

4. Tugwell $P$, Bombardier $C$, Buchanan W, et al. Impact on quality of life assessed by traditional standard-item and individualized patient preference health status questionnaires. Arch Intern Med 1990; 150:59-62.

5. Tugwell P, Bombardier C, Buchanan WW, Goldsmith CH, Grace E, Hanna B. The MACTAR patient preference disability questionnaire: an individualized functional priority approach for assessing improvement in physical disability in clinical trials in rheumatoid arthritis. J Rheumatol 1987; $14: 446-51$.

6. Boers M, Verhoeven AC, Markusse HM, et al. Randomised comparison of combined step-down prednisolone, methotrexate and sulphasalazine with sulphasalazine alone in early rheumatoid arthritis. Lancet 1997; 350:309-18.

7. Amett FG, Edworthy SM, Bloch DA, et al. The American Rheumatism Association revised criteria for rheumatoid arthritis. Arthritis Rheum 1988; 31:315-24.

8. Siegert CEH, Vleming LJ, Vanderbroucke JP, Cats A. Measurement of disability in Dutch rheumatoid arthritis patients. Cin Rheumatol 1984; 3:305-9.

9. Meenan RF, Mason JH, Anderson JJ, Guccione AA, Kazis LE. AIMS2, the content and properties of a revised and expanded arthritis measurement scales health status questionnaire. Arthritis Rheum 1992; 35:1-10.

10. Borstlap M, van de Laar M, Zant J, van der Korst J. Components of health: an analysis in rheumatoid arthritis using quality of life questionnaires and clinical and laboratory variables. Ann Rheum Dis 1993; 52:650-4.

11. Jones E, Hanley JG, Mooney R, Rand LL, Spurway PM, Eastwood BJ, Jones JV. Strength and hand function in the normal and rheumatoid hand. J Rheumatol 1991; 18:1313-8.

12. The cooperating clinics committee of the American Rheumatism Association. A seven-day variability study of 499 patients with peripheral rheumatoid arthritis. Arthritis Rheum 1965; 8:302-34 .

13. Liang $\mathrm{MH}$, Fossel $\mathrm{AH}$, Larson MG. Comparison of five health status instruments for orthopedic evaluation. Med Care 1990; 28:632-42.

14. Beaton BE, Hogg-Johnson S, Bombardier C Evaluating changes in health status: Reliability and responsiveness of five generic health status measures in workers with musculoskeletal disorders. J Cin Epidemiol 1997; 50:79-93.

15. Norman GR, Stratford P, Regehr G. Methodological problems in the retrospective computation of responsiveness to change: The lesson of Cronbach. J Cin Epidemiol 1997; 50:869-79.

16. Bell MJ, Bombardier C, Tugwell P. Measurement of functional status, quality of life, and utility in rheumatoid arthritis. Arthritis Rheum 1990; 33:591-601.

17. Verhoeven AC, Boers M, van der Linden S. Calculating improvement in rheumatoid arthritis by ACR criteria is less straightforward than it looks. Br J Rheum 1997; 36 suppl:151.

18. Wright JG, Young NL. A comparison of different indices of responsiveness. J Cin Epidemiol 1997: 50:239-46.

19. Guyatt G, Berman LB, Townsend N, Taylor DW. Should study objects see their previous responses? J Chron Dis 1985; 38:1003-7. 


\section{Appendix}

\section{MACTAR}

Note: Questions $1 \mathrm{~d}$ and 2 in the follow-up interview are "transitional" questions that measure change. The other questions measure a state; in the article they are referred to as "status" questions.

\section{Baseline interview}

Interviewers note: Read the questions and response categories provided. Tick the response given.

1a. How would you say your overall health has been during the last 2 weeks? You think of it as.

$\begin{array}{ll}\square_{3} & \text { very good } \\ \square_{2} & \text { pretty good } \\ \square_{1} & \text { not too good }\end{array}$

2. Arthritis may cause restrictions in several areas of your daily life. For different people the impact of arthritis is also different. We will ask you to name activities in which you experience difficulties because of your arthritis. What matters here, is what your personal experience has been.

Please, think of what became a problem, now that you have arthritis.

Interviewers note:

In order to elicit a comprehensive list of activities: First, give the patient opportunity to react spontaneously.

Then read the probes. Record the exact phrases of the patient on the lines hereunder.

To support you in naming any problems caused by arthritis, I will read you a number of areas of your daily life that might be affected.

Does your arthritis limit...

- any (other) activities around the house such as cooking, housework etc.?

- any activity related to dressing such as buttoning, pulling a sweater over your head etc.?

- any (other) activities at your work (outside the home), drive a car or other transportation?

- any (other) leisure activities. Either sport such as bowling, swimming, golf, etc. or non-sports such as needlework, woodwork etc.?

- any (other) social activities. Such as visiting, playing cards, going to church etc.?

- sexuality?

Are there changes in the relationship with your family?

If you live together with a husband/ wife/ partner, are there changes in the relation with him/her? If you have children living at home, are there changes in your relationship with them?

\section{The line above is printed 10 times}

Interviewers note: To rank the activities in order of importance to the patient, ask the following questions:

Which of these activities would you most like to be able to do without pain or discomfort of your arthritis? Show and read the list to the patient. Write ' 1 ' next to the activity the patient chose.

After (read activity 1)

which activity would you next most like to be able to do without pain or discomfort of your arthritis?

Show and read the list to the patient, with exception of the activity with priority 1.

Write ' 2 ' next to the activity the patient chose.

After (read activity 1 and 2 )

which activity would you next most like to be able to do without pain or discomfort of your arthritis?

Show and read the list to the patient, with exception of the activity with priority 1 and 2 .

Write ' 3 ' next to the activity the patient chose.

Continue like this, until all activities are ranked.

The 5 with the highest priority will return in the follow-up interview.

3a. In general, how satisfying do you find the way you spend your life ? Over the last week you think of it as...

3b. Is your life not completely satisfying
because of your arthritis?
$\square_{0}$ yes
$\square_{1} \quad$ no

4a. How would you say your overall physical functioning has been ? Over the last week you think of it as...

4b. Is your physical function not as good as it might be because of your arthritis? not very satisfying

$\begin{array}{ll}\square_{5} & \text { good } \\ \square_{4} & \text { fair to good } \\ \square_{3} & \text { fair } \\ \square_{2} & \text { fair to poor } \\ \square_{1} & \text { poor }\end{array}$


5a. How would you say your overall social functioning has been ? Over the last week you think of it as...

5b. Is your social functioning not as well as it might be

$\begin{array}{ccccc}\text { because of your arthritis? } & \square_{0} & \text { yes } & \square_{1} & \text { no }\end{array}$

6a. How would you say your overall emotional functioning has been ? Over the last week you think of it as ...

6b. Is your emotional functioning not as well as it might be

$\begin{array}{ll}\square_{5} & \text { good } \\ \square_{4} & \text { fair to good } \\ \square_{3} & \text { fair } \\ \square_{2} & \text { fair to poor } \\ \square_{1} & \text { poor }\end{array}$

? good

$\mathrm{a}_{4}$ fair to good

$\mathrm{O}_{3}$ fair

$\mathrm{O}_{2}$ fair to poor

$\square_{1}$ poor $\rightarrow$ go to Q6a

$\rightarrow$ stop here
because of your arthritis?
$\square_{0}$ yes
$\square_{1}$ no

\section{Follow-up interview}

1a. How would you say your overall health has been during the last 2 weeks ? You think of it as...

$$
\begin{array}{ll}
\square_{3} & \text { very good } \\
\square_{2} & \text { pretty good } \\
\square_{2} & \text { not too good }
\end{array}
$$

1b. Have you noticed any change in your arthritis since we talked during the first interview?

1c. Please describe how your arthritis has changed ?

$$
\square \text { yes } \square \text { no } \rightarrow \text { 'no change' at Q1d, go to Q2a }
$$

1d. When you think of your arthritis during the two weeks before the first interview. How much better or worse overall has your arthritis become?

$$
\begin{array}{ll}
\square_{7} & \text { a great deal better } \\
\square_{6} & \text { moderately better } \\
\square_{5} & \text { slightly better } \\
\square_{4} & \text { no change } \\
\square_{3} & \text { slightly worse } \\
\square_{2} & \text { moderately worse } \\
\square_{1} & \text { a great deal worse }
\end{array}
$$

You may remember the first time we spoke. You told me which activities were at that time problems due to your arthritis. I will ask you again about the five most important.

2a. Since the first interview, have you noticed any change in your ability to (activity 1 )?
It is ...
$\square_{3}$ less of a problem
$\square_{2}$ the same
$\square_{1}$ more of a problem

2b. Since the first interview, have you noticed any change in your ability to (activity 2 )?
It is ...
$\mathrm{Q}_{3}$ less of a problem
$\square_{2}$ the same
$\square_{1}$ more of a problem

2c. Since the first interview, have you noticed any change in your ability to (activity 3 )?
It is ...
$\square_{3}$ less of a problem
$\square_{2}$ the same
$\square_{1}$ more of a problem

2d. Since the first interview, have you noticed any change in your ability to (activity 4 )? It is

$$
\begin{array}{ll}
\square_{3} & \text { less of a problem } \\
\square_{2} & \text { the same } \\
\square_{1} & \text { more of a problem }
\end{array}
$$

2e. Since the first interview have you noticed any change in your ability to (activity 5 )? It is...

$$
\begin{array}{ll}
\square_{3} & \text { less of a problem } \\
\square_{2} & \text { the same } \\
\square_{1} & \text { more of a problem }
\end{array}
$$

Questions $3 a$ to $6 \mathrm{~b}$ are equal to the equally numbered questions in the baseline interview. 


\section{CHAPTER 12}

\section{SUMMARY AND \\ GENERAL DISCUSSION}

Arco Verhoeven 


\section{Summary and general discussion}

The systematic review in chapter 2 summarizes the many developments in the combined drug treatment of rheumatoid arthritis (RA). We concluded that in early RA patients, step-down bridge therapy with oral corticosteroids leads to enhanced efficacy at acceptable or low toxicity (see Chapter 3). In patients with established disease, cyclosporin improves suboptimal clinical response to methotrexate (MTX) [1], and the triple combination of MTX, sulphasalazine (SSZ), and hydroxychloroquine appears to be clinically better than these single components [2]. Other combinations are either untested, tested at low sample size, or show negative interaction. In view of the low volume of evidence, it was stated that most studies needed confirmation by replication. Since then, confirmation for the usefulness of corticosteroids in early and active disease has been provided by the FINRACO study [3,4]. An important issue is the alleged progression of joint damage after corticosteroid therapy has been withdrawn [5]. Hickling et al found progression resumed once the low-dose prednisone added to standard disease-modifying anti-rheumatic drug (DMARD) therapy was stopped [5,6]. Other researchers even speak of an rebound effect which might implicate that patients are in the end worse off due to the use of steroids before [7]. Data from the COBRA trial did not confirm such an effect, on the contrary, the protective effects of combined-therapy - presumably the prednisolone component in it - persisted up to 3 years after their withdrawal according to trial protocol [8].

The randomised clinical COBRA trial in chapter 3 shows the value of intensive combination therapy by comparing the clinical outcomes of combined step-down prednisolone, MTX and SSZ with SSZ alone in patients with early and active RA. Combined therapy immediately suppressed damage progression, whereas SSZ did less effectively and with a lag of 6 to 12 months. Notably, there were fewer withdrawals in the combined-therapy than the SSZ group, and these occurred later. The combined-therapy regimen thus offers additional disease control [9] over and above that of SSZ alone and persists for at least a year after corticosteroids are stopped.

The design of the COBRA trial does not allow separate evaluation of MTX and prednisolone effects, as both drugs were part of a triple therapy compared to single SSZ. However, the timely pattern of reduced disease activity - with convergent effects directly after the withdrawal of prednisolone and no change after withdrawal of MTX - suggests that MTX does not have a large protective - nor harmful - effect on bone in addition to the effect that had already been achieved by $7.5 \mathrm{mg} /$ day prednisolone and $2000 \mathrm{mg} /$ day SSZ. This view is supported by findings of Haagsma et al's trial with no significant difference in clinical or radiological outcomes between treatment groups with SSZ, MTX or their combination [10]. 
Presently, other studies and long-term follow-up are available to prove the soundness of aggressive treatment of early and active rheumatoid arthritis as well as the persistence of beneficial effects over many years $[5,6,8]$. Notably, the follow-up of patients in the COBRA trial shows an persisting beneficial effect on progression of joint damage as visible on radiographs; 3 and more years after medication dictated by the study protocol has been stopped there are still significant differences in progression of RA related joint damage (erosions in small joints of hands and feet). Analysis on follow-up data on erosion scores show regression lines per treatment group that diverge, instead of converge as might be expected with shrinking between-group differences in medication after withdrawal of short-term trial medication by protocol [8].

Recently, a new class of biological anti-tumor necrosis factor alfa (anti-TNF $\alpha$ ) has come available with drugs named: etanercept and infliximab. These are a recombinant human TNF receptor and a chimeric human-murine monoclonal TNF antibody that bind to and thereby inhibits the biological activity of TNF $\alpha$. Corticosteroids are presumably less precise in their action' as they inflict a series of systemic effects - including adverse like osteoporosis. The first trials with etanercept and infliximab used in combination are published [11,12]. The results are very promising but high costs and concerns about toxicity profiles presently limit their use. Postmarketing surveillance is important as various serious adverse effects of etanercept and infliximab have been reported [13-15]. With others we urge the scientific community to compare the effects and (long-term) toxicity of parental anti-TNF $\alpha$ factors with those of oral corticosteroids in initially high doses [16]. The COBRA medication protocol might serve as a model for this 'aggressive' corticosteroid approach [17].

Chapter 4 demonstrates the cost-effectiveness and cost-utility of early intervention in RA patients, with combined step-down prednisolone, MTX and SSZ, compared to SSZ alone. This conclusion is based on enhanced efficacy at lower or equal direct costs: Cinical, radiographic and functional outcomes significantly favored combined treatment at week 28 (radiography also at week 56 and 80 ) and utility scores measured by rating scale were significantly better at week 28 . Based on, among other factors, fewer days spent in the hospital, direct costs of combined therapy were lower, although the level of significance was not reached. Notably, this is a regular phenomena in - so-called 'piggy-back' - economic evaluations linked to clinical trials; a priori power calculations based on clinical outcomes, and a skewed distribution of costs (caused by a small number of patients with extreme high costs) often have make it hard to reveal more than a 'trend' in lower cost with one of the treatment options under evaluation [18]. Moreover, the narrow timeframe of just over one year made it also difficult to find a between-treatment group differences in costs and QALYs. Nevertheless, our report was the first state-of-the-art costeffectivity analysis of therapy for rheumatoid arthritis based on prospective data (previous studies were based on models or rates instead of 'real-time' measurements and costprices) [19]. We were nor the first nor the only research group to conclude that in patients with a chronic condition (such as arthritis) utility scores measured with the rating scale technique are more responsive than with standard gamble [20]. Indirect costs were not included in this first publication but the trial design comprised patient dairies and questionnaires to evaluate indirect costs (manuscript submitted; Korthals-de Bos IBC et al). 
After initially positive experiences with corticosteroids for RA during the fifties, the recognition of important side effects of high doses and lower doses in established disease caused a communis opinio that this should not be done. However, these observations may be biased by the fact that corticosteroids are usually prescribed in patients with established and therapy-resistant disease. These patients are especially vulnerable to all kind of adverse effects that are associated with corticosteroids. Corticosteroids can play an important role in the reduction of disease activity in RA patients with early and active disease as illustrated by the COBRA trial as well as other studies. Therefore they should not be considered as a class of drugs that merely reduce symptoms but as DCARDs; disease-controlling antirheumatic drugs [9]. The systematic review in chapter 5 showed that bone loss due to corticosteroids in the spine and hips of RA patients is in general more limited than in patients with other diseases. This conclusion was based on data from cohorts in papers published between 1966 and 1995 but is confirmed and accepted by recent publications $[21,22]$. Non-RA patients on high doses of corticosteroids regularly lose clinically relevant amounts of bone (arbitrarily set at $5 \%$ within 1 year), but this is rare in patients with RA. Most bone is lost in early or uncontrolled disease. Disease control with corticosteroids neutralises - at least partially - the corticosteroid induced bone loss. Patients in the combination therapy group of the COBRA trial - to whom $2345 \mathrm{mg}$ prednisolone was subscribed during 28 weeks - 8 of 64 patients lost more than $5 \%$ of spinal bone (versus 6 of 62 in the SSZ group). Mean bone loss in the lumbar spine was indeed larger in the combination group $(P=0.06)$ but this stabilized after the withdrawal of prednisolone.

In RA the pathological destruction of collagen in bone and cartilage, causes the crosslink bridges in mature collagen to be resorbed more rapidly than normal. These collagen crosslinks are excreted in urine. Apart from the crosslink resorption at the site of inflamed joints, there might be increased resorption due to general bone loss associated with RA disease activity. Important collagen crosslinks and markers of destruction of bone and cartilage are pyridinoline (PYD) and deoxypyridinoline (DPD). Because of circadian rhythm in bone remodelling excretion in 24-hours collection is the standard. The findings described in chapter 6 support that in longitudinal studies also urinary spot samples collected during a fixed day time (with concentrations corrected for creatinine) reflect 24-hour excretion levels well. In chapter 7 we conclude from the available data in the COBRA trial, that prednisolone and DMARD therapy in these patients with early and active RA were both independently associated with decreased levels of urinary excretion of the bone collagen resorption markers PYD and DPD. In early RA the markers of bone formation and resorption closely followed changes in disease activity measure such as ESR in both treatment groups [23]. Reduced bone resorption together with reduced bone formation - reduction of formation initially at a somewhat faster pace - resulted in less bone turnover and may explain the observed small and partially reversible extra bone loss in the lumbar spine associated with prednisolone. 
Chapter 8 confirms that patients with longer disease duration do not respond as well to treatment in RA, and female gender, prior DMARD use, ARA class and disease activity also have effects on the likelihood of patient response to treatment. This has implications for trial interpretation and for clinical expectations of patients. It also supports the view that there is a 'window of opportunity' in the treatment of RA. Aggressive suppression of disease activity in the early phase of active disease (with apparent inflammation of small joints of hands and feet) is mandatory to prevent joint damage. Association of cumulative disease activity with joint erosions and function loss at later stages stresses the importance of disease duration. The opportunity to intervene early with second-line antirheumatic drugs relies heavily on the early diagnosis and rapid referral of patients; health care systems should be specifically organized to facilitate this process [24].

The analysis described in chapter 9 focussed on the validation of responsiveness and discriminative power of the World Health Organization/ International League of Associations for Rheumatology (WHO/ILAR) core set, together with the American College of Rheumatology (ACR) and European League Against Rheumatism (EULAR) criteria for improvement/response, and other single and combined measures (indices) in a trial in patients with early RA. It shows that at the moment of maximum between-group difference standardized response means (as sign for responsiveness) for various core set measures differs largely. Performance of patient oriented measures (for example, pain, global assessment) is best when the questions were focused on the disease. The most responsive single measure is the patient's assessment of change in disease activity. Patient utility, a generic health status measure was moderately (rating scale) to poorly responsive (standard gamble). Combined measures (indices) no matter how simple did better than most single measures; the simple count of core set measures improved by $20 \%$ was the most responsive. Discrimination performance yielded similar but not identical results: best discrimination between treatment groups was achieved by the EULAR response and ACR improvement criteria (at 20\% and other percentage levels), the pooled index, and the disease activity score (DAS), but also by the Health Assessment Questionnaire (HAQ) and grip strength. We concluded that responsiveness and discrimination between levels of response are not identical concepts. As a matter of fact they need separate study. The WHO/ILAR core set comprises responsive measures that discriminate well between different levels of response in early RA. However, the performance of patient oriented measures is highly dependent on their format. The excellent performance of indices such as the ACR improvement and EULAR response criteria confirms they are the preferred primary endpoint in RA clinical trials.

When ACR improvement criteria are applied, measured scores should decrease on improvement. This, not only on the basis of methodological considerations (illustrated by data examples from the COBRA trial in chapter 10 of this thesis) but also to facilitate comparisons between trials. Where necessary, raw data should be recoded before the ACR criteria are applied. This advice seems to gain importance with thresholds for improvement larger then the classical $20 \%$. 
The MACTAR interview evaluated in chapter 11 (indeed an interview rather than a questionnaire) is a valid and highly responsive instrument to assess change in functional ability of early RA patients with active disease. The items that directly address change were among the most responsive of all. The MACTAR interview provides insight into problems - mainly of physical function - that really matter to patients. The feasibility of the MACTAR in standard clinical trials as well as clinical care, is considered to be limited compared with widely used instruments for physical disability such as the Health Assessment Questionnaire [25].

Patients that are willing to participate in clinical studies are rare and in almost every instance more rare than was expected. Our patients were prepared to be interviewed, touched, squeezed, and punctured. For this they deserve compassion and deepest respect; their participation is an act of philanthropy aimed at the benefit of future fellow-patients. In this context, valid and sensitive measurement, sound methodology, and careful and 'economic' use of eligible patients should be a matter of course. The quest for - the most - valid and responsive measures should never stop. Both disease-specific and generic measures of function and health-related quality of life are capable to detect improvements in RA patients. Using both types of measures for evaluating therapies will identify discernible changes that are important to patients, and will facilitate comparisons across different disease states [26]. 


\section{References}

1. Tugwell P, Pincus T, Yocum D, Stein M, Gluck O, Kraag G, McKendry R, Tesser J, Baker P, Wells G. Combination with cyclosporine and methotrexate in severe rheumatoid arthritis. N Engl J Med 1995; 333:137-41.

2. ODell JR, Haire CE, Erikson N, Drymalski W, Palmer W, Eckhoff PJ, Garwood V, Maloley P, Klassen LW, Wees S, Klein H, Moore GF. Treatment of rheumatoid arthritis with methotrexate alone, sulfasalazine and hydroxychloroquine, or a combination of all three medications. NEngl J Med 1996; 334: 1287-91.

3. Möttönen T, Hannonen P, Leirisalo-Repo M, Nissila M, Kautiainen H, Korpela M, Laasonen L, Julkunen H, Luukkainen R, Vuori K, Paimela L, Blafield H, Hakala M, Ilva K, Yli-Kerttula U, Puolakka K, Jarvinen P, Hakola M, P̈̈rainen H, Ahonen J, Palvimaki I, Forsberg S, Koota K, Friman C. Comparison of combination therapy with single-drug therapy in early rheumatoid arthritis: a randomised trial. FIN-RACo trial group. Lancet 1999; 353:1568-73..

4. Neva MH, Kauppi MJ, Kautiainen H, Luukkainen R, Hannonen P, Leirisalo-Repo M, Nissila M, Möttönen T. FIN-RACo Trial Group Combination drug therapy retards the development of rheumatoid atlantoaxial subluxations. Arthritis Rheum 2000; 43:2397-401.

5. Hickling P, Jacoby RK, Kirwan JR Joint destruction after glucocorticoids are withdrawn in early rheumatoid arthritis. Arthritis and Rheumatism Council Low-dose Glucocorticoid Study Group. Br J Rheumatol 1998; 37:930-6.

6. Kirwan JR. The effect of glucocorticoids on joint destruction in rheumatoid arthritis. Arthritis and Rheumatism Council Low-dose Glucocorticoid Study Group. N Engl J Med 1995; 333:142-6.

7. van Gestel AM, Laan RF, Haagsma CJ, van de Putte LB, van Riel PL. Oral steroids as bridge therapy in rheumatoid arthritis patients starting with parenteral gold. A randomized double-blind placebocontrolled trial. Br J Rheumatol 1995; 34:347-51

8. Landewé RB, Boers M, Verhoeven AC, Westhovens R, van de Laar MA, Markusse HM, van Denderen JC, Westedt ML, Peeters AJ, Dijkmans BA, Jacobs P, Boonen A, van der Heijde DM, van der Linden S. COBRA combination therapy in patients with early rheumatoid arthritis: Long-term structural benefits of a brief intervention. Arthritis Rheum 2002; 45:347-56.

9. Edmonds JP, Scott DL, Furst DE, Brooks P, Paulus HE. Antirheumatic drugs: a proposed new classification. Arthritis Rheum 1993; 36:336-9.

10. Haagsma CJ, van Riel PLCM, de Jong AJL, van de Putte LBA. Combination of sulphasalazine and methotrexate versus single components in early rheumatoid arthritis: a randomized, controlled, double blind, 52 week clinical trial. Br J Rheumatol 1997; 36:1082-8.

11. Moreland LW, Schiff MH, Baumgartner SW, Tindall EA, Fleischmann RM, Bulpitt KJ, Weaver AL, Keystone EC, Furst DE, Mease PJ, Ruderman EM, Horwitz DA, Arkfeld DG, Garrison L, Burge DJ, Blosch CM, Lange ML, McDonnell ND, Weinblatt ME. Etanercept therapy in rheumatoid arthritis. A randomized, controlled trial. Ann Intern Med 1999; 130:478-86.

12. Bathon JM, Martin RW, Fleischmann RM, Tesser JR, Schiff MH, Keystone EC, Genovese MC, Wasko MC, Moreland LW, Weaver AL, Markenson J, Finck BK. A comparison of etanercept and methotrexate in patients with early rheumatoid arthritis. NEngl J Med 2000; 343:1586-93.

13. Lipsky PE, van der Heijde DM, St Cair EW, Furst DE, Breedveld FC, Kalden JR, Smolen JS, Weisman M, Emery P, Feldmann M, Harriman GR, Maini RN. Infliximab and methotrexate in the treatment of rheumatoid arthritis. Anti-Tumor necrosis factor Trial in Rheumatoid Arthritis with Concomitant Therapy study group. N Engl J Med 2000; 343:1594-602.

14. European Agency for the Evaluation of Medicinal Products: Revised EMEA public statement on etanercept (Enbrel) - serious hematological reactions and demyelination disorders. Oct 3, 2000, EMEA/H/30871/. (www.emea.eu.int/pdfs/human/press/pus/3087100en).

15. European Agency for the Evaluation of Medicinal Products: EMEA public statement on infliximab (Remicade) - reports of tubercolosis infections. Dec 20, 2000, EMEA/CPMP/4445/00. (www.emea.eu.int/pdfs/human/press/pus/444500en). 
16. Smith MD, Ahem MJ, Roberts-Thomson PJ, Youssef PP. Similar effects of pulse corticosteroid and tumor necrosis factor $\alpha$ blockade in rheumatoid arthritis: comment on the article by Taylor et al. Arthritis Rheum 2001; 44:25.

17. Goekoop YPM, Allaart CFR, Breedveld FC, Dijkmans BAC. Combination therapy in rheumatoid arthritis. Gur Opin Rheumatol 2001; 13:177-83.

18. Groot CA, Touw CR. Economic evaluation of cancer treatments: methodological treatment: methodological and practical issues. Anticancer Drugs 1998; 9:835-41.

19. Bosi Ferraz M, Maetzel A, Bombardier C. Critical appraisal of economic evaluation published in the field of rheumatology and related disciplines. Arthritis Rheum 1997; 40:1587-93.

20. Goossens ME, Vlaeyen JW, Rutten-van Mölken MP, van der Linden SM. Patient utilities in chronic musculoskeletal pain: how useful is the standard gamble method? Pain 1999; 80:365-75.

21. Solomon DH, Kuntz KM. Should postmenopausal women with rheumatoid arthritis who are starting corticosteroid treatment be screened for osteoporosis? A cost-effectiveness analysis. Arthritis Rheum 2000; 43:1967-75.

22. Criswell LA, Saag KG, Sems KM, Welch V, Shea B, Wells G, Suarez-Almazor ME. Moderate-term, low-dose corticosteroids for rheumatoid arthritis. Cochrane Database Syst Rev 2000; 2: CD001158.

23. Westhovens R, Dequeker J. Rheumatoid arthritis and osteoporosis. Z Rheumatol 2000; 59 suppl:33-8.

24. van der Horst-Bruinsma IE, Speyer I, Visser H, Breedveld FC, Hazes JM. Diagnosis and course of early-onset arthritis: results of a special early arthritis clinic compared to routine patient care. Br J Rheumatol 1998; 37:1084-8.

25. Pincus T, Wolfe F. An infrastructure of patient questionnaires at each rheumatology visit: improving efficiency and documenting care. J Rheumatol 2000; 27:2727-30.

26. Tugwell P, Wells G, Strand V, Maetzel A, Bombardier C, Crawford B, Dorrier C, Thompson A. Leflunomide Rheumatoid Arthritis Investigators Group. Cinical improvement as reflected in measures of function and health-related quality of life following treatment with leflunomide compared with methotrexate in patients with rheumatoid arthritis: sensitivity and relative efficiency to detect a treatment effect in a twelve-month, placebo-controlled trial. Arthritis Rheum 2000; 43:506-14. 


\section{SAMENVATTING}




\section{Samenvatting}

Reumatoïde artritis (RA) is een veel voorkomende aandoening met onbekende oorzaak die gekenmerkt wordt door een chronische ontsteking van de kleine gewrichten van handen en voeten. De patiënt of patiënte - de meeste patiënten zijn vrouw - heeft pijnlijke en gezwollen gewrichten, er is ochtendstijfheid en vermoeidheid. Ook op andere manieren kan de ziekte zich manifesteren, bijvoorbeeld met reumaknobbeltjes of bloedvatontsteking. Soms is het beloop van de ziekte vrij mild en gaat het als vanzelf beter met de patiënt maar meestal wisselen goede en slechte perioden elkaar af. Vaak zijn na verloop van tijd - soms al kort nadat de ziekte is vastgesteld - afwijkingen te zien op röntgenfoto's; gaatjes in het bot bij de kleine gewrichten. Pijn, zwelling en stijfheid zijn uitingen die wel beschreven worden als de ziekteactiviteit; naarmate deze heviger is en langer bestaat, is het waarschijnlijker dat meer schade aan de gewrichten optreedt. Op den duur ontstaan de kenmerkende en invaliderende standsafwijkingen. De ziekte bedreigt de kwaliteit van leven van patiënten en vermindert zelfs hun levensverwachting.

De behandeling van RA bestaat van oudsher uit leefregels, fysiotherapie en ontstekingsremmende middelen uit de groep van zogenaamde niet-sterö̈de anti-inflammatoire middelen (NSAIDs). Deze medicijnen worden beschouwd als relatief onschuldig maar zij kunnen het reumatoïde ontstekingproces niet werkelijk remmen. Wel hebben zij een effect op pijn en stijfheid.

Bij patiënten die onvoldoende baat vinden bij de ingestelde therapie wordt behandeling gestart met een tweedelijns antireumatica (geneesmiddelen zoals goud, sulfasalazine en methotrexaat). Deze middelen hebben soms ernstige bijwerkingen maar zij kunnen wel de schade afremmen die de reumatoïde ziekteactiviteit aanricht. De werking van deze middelen laat vaak enige tijd op zich wachten. In het geval van bijwerkingen of - na verloop van tijd - bij vaststelling van onvoldoende effect, wordt een ander middel geprobeerd. Patiënten met de meer hardnekkige vormen van RA werden aldus over jaren behandeld met steeds andere, maar ook steeds zwaardere middelen; men spreekt van de therapeutische piramide (zie figuur).

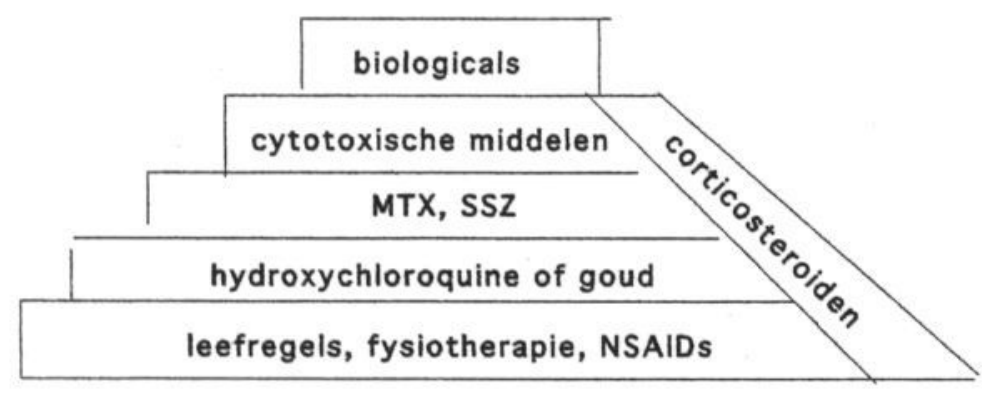

Figuur: De klassieke therapeutische piramide voor de behandeling van reumatoïde artritis 
Corticosteroïden vormen een aparte groep van middelen die veel gebruikt wordt in de reumatologie. Kort na de introductie in de vijftiger jaren was het enthousiasme groot. Met het bredere gebruik werden ook de bijwerkingen duidelijker. Onder het publiek is vrij algemeen bekend dat bijvoorbeeld het middel prednison is verbonden met gewichtstoename en botontkalking. Hoewel corticosteroïden veel worden gebruikt bij met name langer bestaande RA, menen veel reumatologen dat zij alleen ingezet mogen worden als een laatste redmiddel.

Eind jaren negentig groeide het ongenoegen onder reumatologen over de traditionele behandeling van RA. Het werd duidelijk dat de hevigheid van de reumatoïde ontstekingen samenhangt met de schade die de ziekte aanricht aan de gewrichten. Tevens bleek dat de meeste beschadigingen in de eerste ziektejaren optreden - vooral bij patiënten met een hevige ziekteactiviteit. Met de voorzichtige stap voor stap strategie gaat veel tijd verloren om een therapie te vinden die min of meer adequaat de ziekteactiviteit kan onderdrukken. Omdat behandelaars het idee hadden in veel gevallen achter de feiten (lees gewrichtsschade) aan te lopen ontstond de roep om sneller te behandelen met zwaardere middelen en met combinaties.

Door medicijnen te combineren hoopte men effecten te bereiken die even groot, of zelfs groter zouden zijn dan de opgetelde effecten van de middelen afzonderlijk. Ook hoopte men op minder bijwerkingen lagere doses te gebruiken. Hoewel diverse combinatie-therapieën reeds frequent werden toegepast ontbrak het wetenschappelijk bewijs ter rechtvaardiging van deze praktijk nog. Het beperkte onderzoek dat wel was gedaan gaf weinig reden tot optimisme en met name over de rol van corticosteroïden in combinatie met antireumatica bestond onduidelijkheid.

De basis voor dit proefschrift werd gelegd binnen Ontwikkelingsgeneeskunde. Dit initiatief van de Ziekenfondsraad was gericht op het beoordelen van de waarde van nieuwe technieken of benaderingen op het gebied van diagnostiek of therapie te evalueren. Evaluatie van kosteneffectiviteit maakte een integraal onderdeel uit van alle projecten. In ons project werd de werkzaamheid onderzocht van combinatietherapie (met tweedelijns antireumatica) bij vroege reumatoïde a artritis (het COBRA-onderzoek). Het geven van hoge doses prednisolon bij patiënten met kort bestaande RA, was op het moment van starten van het onderzoek uiterst controversieel.

Dit proefschrift bevat 10 hoofdstukken die eerder als artikelen in internationale medische vaktijdschriften gepubliceerd zijn. Na de algemene inleiding (hoofdstuk 1) volgen drie delen:

- Het eerste deel omvat een systematisch overzicht van onderzoeken naar combinatietherapie met tweedelijns antireumatica bij RA. Daarna handelen twee hoofdstukken over de klinische uitkomsten en kosten-effectiviteit van de combinatie-therapie zoals die toegepast is binnen het COBRA-onderzoek (hoofdstukken 2, 3 en 4).

- Het tweede deel handelt over de invloeden van corticosteroïden op de botstofwisseling bij RA-patiënten (hoofdstukken 5, 6 en 7).

- Het derde deel gaat onder andere over onderzoekstechnische aspecten van het vaststellen van uitkomsten bij RA-patiënten (hoofdstukken 8, 9, 10 en 11). 
Het beperkte aantal beschikbare antireumatica biedt gelegenheid tot een enorme diversiteit van combinaties. Hoofdstuk 2 geeft een systematisch overzicht van hoge-kwaliteit onderzoeken naar combinatietherapie bij RA. In patiëntengroepen met kort bestaande RA bleek de combinatietherapie uit het COBRA-onderzoek (zie hoofdstuk 3) de klinische uitkomsten belangrijk te verbeteren zonder onacceptabele bijwerkingen. Bij patiënten met langer bestaande RA bleek het middel cyclosporine gegeven naast methotrexaat de klinische uitkomsten te verbeteren. Ook de combinatie van methotrexaat, sulfasalazine en hydroxychloroquine liet betere klinische uitkomsten zien dan elk van deze 3 middelen afzonderlijk. Veel combinaties zijn niet onderzocht, andere bleken te zijn getoest in te kleine groepen, of de combinaties toonden een effect dat geringer was dan verwacht werd van elk van de middelen afzonderlijk.

In het COBRA-onderzoek beschreven in hoofdstuk 3 zijn twee groepen van patiënten vergeleken. Eén groep kreeg de combinatie van drie middelen: sulfasalazine, methotrexaat en prednisolon. De andere groep kreeg alleen sulfasalazine. Prednisolon werd na 28 weken gestopt, methotrexaat na 40 weken. De ziekteactiviteit (ontstoken gewrichten, bloedbezinking, etc.) verminderde in de groep met combinatietherapie vrijwel onmiddellijk; de verbetering was bijna tweemaal zo groot als die door alleen sulfasalazine. $\mathrm{Na}$ het stoppen van prednisolon nam het verschil tussen de groepen af (en was niet langer significant). Het stoppen van methotrexaat (het tweede middel uit de combinatie) deed vervolgens niets toe of af aan het verschil. Uit het onderzoek van röntgenfoto's van handen en voeten bleek dat in de groep met combinatietherapie veel minder nieuwe gewrichtschade was ontstaan. Dit significante verschil bestond na een half jaar combinatietherapie maar bleek te blijven bestaan tot tenminste een jaar na het stoppen van de prednisolon. Er werden geen ernstige bijwerkingen gezien in de patiëntengroep met combinatietherapie; het aantal uitvallers was gering en significant minder dan in de sulfasalazinegroep. Ook de verwachte botontkalking door prednisolon bleek gering.

Verrassend genoeg bleek bij het volgen van de patiënten en hun röntgenfoto's (tot meer dan 4 jaren na aanvang van het onderzoek) dat de verschillen in gewrichtsschade tussen de oorspronkelijke twee behandelgroepen bleven bestaan. Dit duidt op de noodzakelijkheid van het vroegtijdig onderdrukken van de ziekteactiviteit; zonder deze harde aanpak gaat de kans op voorkómen van gewrichtsschade voorbij! Sinds kort is voor de behandeling van RA een nieuwe klasse medicijnen beschikbaar: anti-TNF $\alpha$-middelen (in het Engels ook wel aangeduid als 'biologicals'). De extreem hoge kosten en onzekerheid over bijwerkingen op korte en lange termijn maken ruime toepassing van deze middelen vooralsnog problematisch. Een directe vergelijking van de effecten van deze middelen met die van corticosteroïden ligt voor de hand.

De financiële gevolgen van RA zijn groot door o.a. medische kosten en arbeidsongeschiktheid. Bij analyse van de behandelkosten beschreven in hoofdstuk 4 bleek de combinatietherapie uit het COBRA-onderzoek iets goedkoper. De extra medicatie en verrichtingen voor controle van o.a. botdichtheid resulteerden in een bescheiden toename van de kosten, maar daar tegenover stonden besparingen in o.a. doktersbezoek en ziekenhuisopnames. De vergelijkbare kosten en superieure effectiviteit samen maken dat combinatietherapie meer kosten-effectief is dan alleen sulfasalazine - bij patiëntengroepen zoals in dit onderzoek. 
Het tweede deel van dit proefschrift met de hoofdstukken 5, 6 en 7 draait om de invloeden van corticosteroïden op botstofwisseling bij RA-patiënten.

In hoofdstuk 5 is gepoogd de invloed van corticosteroïden op botdichtheid uit te drukken in maat en getal. Uit de gecombineerde gegevens van prospectieve onderzoeken bleek de door corticosteroïden veroorzaakte botontkalking in de wervelkolom en heupen van RA-patiënten doorgaans beperkter dan bij andere ziekten. Bij RA treedt het meeste botverlies op vroeg in het beloop van de ziekte en bij hoge ziekteactiviteit. Corticosteroïdengebruik leidt enerzijds tot een verminderde botdichtheid, deze wordt echter anderzijds tenminste gedeeltelijk gecompenseerd door een betere beheersing van de ziekteactiviteit.

Bij RA is er ontsteking van de gewrichtskapsels met de kenmerkende botontkalking rond de gewrichten. Ook in de rest van het skelet is de botstofwisseling veranderd door RA. Diverse stofwisselingproducten in het bloed en de urine kunnen een inzicht geven in het niveau van botaanmaak en botafbraak onder invloed van ziekte en medicatie. In hoofdstuk 6 wordt aangetoond dat urinemonsters die zijn verzameld op een vast tijdstip een 24-uurs urineverzameling kunnen vervangen bij de bepaling van merkstoffen van botafbraak. In hoofdstuk 7 is wederom gebruik gemaakt van de gegevens uit het COBRA-onderzoek. Hieruit volgt de conclusie dat gebruik van prednisolon en sulfasalazine beide zijn verbonden met afgenomen uitscheiding van de merkstoffen voor botafbraak. De stofwisseling onder invloed van medicatie lieten een afname zien van zowel botafbraak als botaanmaak vergelijkbaar met de patronen die werden waargenomen bij maten voor ziekteactiviteit. Het voorbijgaande extra botverlies in de wervelkolom in de groep met combinatietherapie (o.a. prednisolon) is te verklaren uit de waarneming dat in deze groep de botaanmaak aanvankelijk meer werd geremd dan de botafbraak.

Het derde en laatste deel van dit proefschrift met de hoofdstukken 8, 9, 10 en 11 gaat onder meer over de onderzoektechnische (methodologische) aspecten van vaststellen van uitkomsten. Omdat er erg veel uitkomstmaten een rol spelen bij RA is dit een ingewikkeld - maar ook interessant onderzoeksveld.

Hoofdstuk 8 gaat over de rol van ziekteduur bij de behandeling van RA. Gegevens van 14 onderzoeken (waaronder het COBRA-onderzoek) zijn gebruikt in een analyse. Naast geslacht, ziekteactiviteit, voorafgaand medicijngebruik en functiebeperking, blijkt ziekteduur bepalend voor de mogelijk bereiken klinische verbeteringen. Ten einde gewrichtschade op termijn te voorkomen is het van eminent belang dat de ziekteactiviteit (die tot uitdrukking komt in onder ander pijnlijke en gezwollen gewrichten) snel wordt onderdrukt met medicijnen. Vroege herkenning en verwijzing van patiënten naar de reumatoloog is noodzakelijk.

Patiënten werven voor een klinisch onderzoek blijkt telkens weer moeilijker dan verwacht, bovendien komen belangrijke onderzoeksresultaten nooit te vroeg. Daarom is het van groot belang - ook in dit opzicht - zuinig om te gaan met patiënten en te kiezen voor onderzoeksmethoden en uitkomstmaten die in zo kort mogelijke tijd en met medewerking van zo weinig mogelijk patiënten leiden tot eenduidige uitslagen. Hoofdstuk 9 bevat een analyse van de eigenschappen van de uitkomstmaten die gebruikt worden in het onderzoek naar RA. Aan de hand van deze analyse kunnen bruikbare en minder bruikbare uitkomstmaten worden onderscheiden. 
Met gegevens uit het COBRA-onderzoek is het vermogen van uitkomstmaten om verbeteringen binnen patiënten en tussen patiëntgroepen op te sporen onderzocht. Samengestelde uitkomstmaten (indices) bleken bijna altijd beter te presteren dan enkelvoudige maten. De meest gebruikte indices voor verbetering bleken goed te voldoen. Bij het bevragen van de patiënt is het beter te informeren naar veranderingen in het ziektebeeld dan naar algemene gezondheid of welbevinden.

$\mathrm{Bij}$ het rapporteren van veranderingen uitgedrukt in percentages, stuitten wij op de haken en ogen die hieraan vastzitten. Dit vormde de aanleiding voor hoofdstuk 10 met de aanbeveling om voorafgaand aan de berekening van relatieve veranderingen, de data zo te bewerken dat scores afnemen wanneer de patiënt vooruit gaat.

Hoofdstuk 11 behandelt de bruikbaarheid en waarde van een vragenlijst die ook ingezet werd bij het COBRA-onderzoek - als één van de 5 belangrijkste uitkomstmaten. Deze door de onderzoekers afgenomen MACTAR-lijst meet veranderingen in functionele beperkingen, die door de patiënten werden benoemd als belangrijk. Uit het onderzoek kwam de MACTAR-lijst naar voren als erg geschikt voor het opsporen van verschillen tussen patiëntgroepen. Daarnaast verschaft hij inzicht in - meest lichamelijke - beperkingen die belangrijk zijn vanuit het gezichtspunt van de patiënt. Voor de alledaagse patiëntenzorg blijft het gebruik van meer gangbare lijsten (bijvoorbeeld de HAQ) aan te bevelen omdat deze eenvoudiger zijn af te nemen en te interpreteren.

Hoofdstuk 12 is de engelstalige samenvatting. Hierin worden conclusies getrokken en aanbevelingen gedaan die - in hopelijk begrijpelijkere termen - ook dit nederlandstalige hoofdstuk zijn te lezen.

\section{Verder lezen ?}

Speyer I, van der Horst-Bruinsma IE, Breedveld FC, Hazes JM. Diagnose en beloop van vroege artritis; onderzoek naar 'early arthritis clinic'. Ned Tijdschr Geneeskd 1996; 140: 882-5.

Boers M, Verhoeven AC, van der Linden S. Combinatietherapie bij vroege reumatoïde artritis: Het COBRA-onderzoek. Ned Tijdschr Geneeskd 1997; 141: 2428-32.

van den Burg MPG, van Loenhout JWA. Combinatietherapie bij reumatoïde artritis (COBRA); rapport uitgebracht door het College voor Zorgverzekeringen aan de minister van Volksgezondheid, Welzijn en Sport d.d. 28 oktober 1999.

Verhoeven AC, Boers M. Ingezonden brief in reactie op: Tijhuis GJ, van de Putte LB, Breedveld FC. Behandeling van reumatoïde artritis door blokkade van tumornecrosisfactor met infliximab of etanercept, (2001: 1880-5), Ned Tijdschr Geneeskd 2001; 145; 2294-5. 
DANKWOORD 


\section{Dankwoord}

Een proefschrift afscheiden over een zo omvangrijk patiëntgebonden onderzoek als het COBRA-project bleek in de praktijk een langdurig proces met menig moment van wanhoop. Hoewel ik het op momenten als een eenzame tocht ervaren heb weet ik dat dat gevoel niet terecht is want zonder de welwillende betrokkenheid van zeer velen was het nooit tot een goed eind gekomen.

Mijn dank gaat op de allereerste plaats uit naar de moedige patiënten die bereid waren deel te nemen. Voor de vooruitgang van mijn particulier of het algemeen wetenschappelijk inzicht waren zij bereid zich te laten prikken, knijpen, en uitgebreid te laten bevragen. Ik dank $U$ allen!

Nella Houtman, dank voor de waardevol gebleken introductie in de reumatologie en het concept van de therapeutische piramide, 11 jaar geleden.

Maarten Boers is de bedenker en projectleider van het COBRA-onderzoek. In oktober 1992 mocht ik op een juist rijdende trein springen om meteen full-time in de locomotief mee te doen; de richting van het spoor lag vast maar waar het zou eindigen was toen nog onduidelijk, belangrijke wissels werden snel genomen en de gang werd er flink ingezet. Achteraf bekeken zijn de bereikte reisdoelen van een onverwachte pracht. Beste Maarten, ik heb veel aan je te danken, het tempo en de kwaliteit van je altijd opbouwende kritiek is ongeëvenaard, ik heb ontelbare zaken van je mogen leren met misschien wel als belangrijkste les; geef nooit op! Ik zal nooit spijt krijgen van mijn keus eertijds in het mooie Maastricht mijn geluk te beproeven.

Mijn promotor Sjef van der Linden vanaf het begin: beste Sjef, op cruciale momenten wist je de juiste toon te treffen om mij het einddoel niet uit het oog te laten verliezen. Dank daarvoor.

Mijn reisgenoten van het 'COBRA-team' waren mij tot grote steun: Marjolein Braeken, onderzoekassistente en collega van het eerste uur, ik heb je grote betrokkenheid bij 'onze' patiënten en ons onderzoeksproject bijzonder gewaardeerd. Lily Heusschen-Houben, secretaresse en data-entry manager: het was een groot voorrecht om met je te mogen werken. Samen waren jullie een meer dan voortreffelijke steun en toeverlaat bij het overkomen van enorme datastromen; ik herinner mij bergen van CRFs, zilvermijnen van röntgenfoto's en gletschers van rap ontdooiende urine- en serummonsters die in de late avonduren in de vreemdste oorden llees ziekenhuizen) zijn overwonnen. Jack Bibo, Carla Bakker en Monique Seuren bedankt voor jullie "kostelijke" en uiterst "utiele" bijdragen!

Op het een gevaar af gewaardeerde reisgenoten te vergeten, ga ik toch proberen allen die direct bij het COBRA-onderzoek waren betrokken te noemen: Mijn grote dank gaat uit naar de reumatologen en onderzoeksassistenten: Joke de Boer en Hans van de Brink in het Medisch Centrum Alk maar; Eugenie Bergmans, Jos van der Burg, Christiaan van Denderen, Lina Heynen, Ina Peyrot en Jan van Willigen in het Jan van Breemen Instituut te $A$ msterdam; André Peeters in het Reinier de Graaf Gasthuis te Delft; Annet ter Avest, Mart van de Laar, Huub Overmars en Diny Somhorst in het Medisch Spectrum Twente te Enschede en het Twenteborg Ziekenhuis te A lmelo; Marie-Louise Westedt en Jendé van Zeben in het Bronovo Ziekenhuis te 's Gravenhage; 
Marian Bertens, Irna Driece, Ben Dijkmans, Mieke Hazes (sic), Nel Plukkel, Annemiek Versluys, Egbert de Vries en Liesbeth van der Weerdt in het Academisch Ziekenhuis Leiden (thans LUMC en in de rest van de 'STROregio'); Violette Demeester, Heidie Fonderie, Goedele Romanus en René Westhovens in het Pellenberg Ziekenhuis van de Katholieke Universiteit te Leuven, België; Lou Cauberg en Alex Essers in het Academisch Ziekenhuis Maastricht; Jolanda Hendriks, Piet Jacobs, en Marga Janssen in het Sint Laurentius Ziekenhuis te Roermond; en Harry Markusse in de Daniël den Hoed Kliniek (thans Zuiderziekenhuis) te Rotterdam. Allen bedankt voor jullie toegewijde en deskundige medewerking.

Ik dank de leden van de veiligheidscommissie Ab Beysens Nol Cats, Hans Rasker en Hubert Schouten voor hun welwillende, kritische en deskundige betrokkenheid bij het onderzoek. Hubert nogmaals en Marion de Leeuw voor de ondersteuning bij randomisatie en statistische analyses. Ik dank de ziekenhuisapothekers en medewerkers van het azM: Monique van der Westerlaken, Francine de Stoppelaar, Tiny Raadschilders, Els van Hulten en Yvonne Gloudemans voor het verzorgen van de gerandomiseerde medicatie. René Wouters bedankt voor botdichtheidsmetingen bij de Maastrichtse patiënten. Medewerkers van afdelingen repro, fotografie en financiën in het azM bedankt. Ella van de Berg-Loonen en medewerkers van Weefseltypering in het azM, Gertjan van der Sluys Veer en Ellen Kirsten van het Klinisch Laboratorium in het Medisch Spectrum Twente, Jan Steggink in het Twenteborgziekenhuis, Johan te Koppele, Jeroen de Roos en Willemijn van der Laan van TNO-PG, Leiden en de medewerkers van het Reumatologie-lab in het LUMC, dank voor het uitvoeren van talloze bepalingen. Paul van Hove uit Sint Gillis-Waas en zijn medewerkers, bedankt voor het bouwen en onderhouden van onze imposante relationele database.

Annelies Boonen en Désirée van der Heijde wil ik speciaal bedanken voor hun grote betrokkenheid bij (het trainen en uitvoeren van) de röntgenologische scoring van gewrichtsschade. Ook andere (ex-)medewerkers, arts-assistenten en onderzoekers van de werkgroep reumatologie: Peggy Remkins-Lampkin, Yolanda Soons, Marijke van Santen-Hoeufft, Paulien Bolwijn, Alita Hidding, Ineke Blaauw, Zuzana de Jong, Christine Langenaken, Peter Dubbeld, Liesbeth Hoefft, Erik de Klerk, Simone Gorter, Piet Geusens, Robert Landewé en Karin Bruynesteyn. Bedankt voor de collegiale en stimulerende werkomgeving die jullie mij boden.

Leden van de beoordelingscommissie: Professoren van de Brandt, van Engelshoven, Hazes, en Severens; zeer bedankt voor uw bereidheid om mijn werk te beoordelen.

Paranimfen Anneke Spoorenberg, lieve collega forever to be, en Roesja, allerliefste zus, bedankt dat jullie deze rol op jullie willen nemen nu deze reis bijna is volbracht.

Lieve familie, vrienden en (ex-)collega's, bedankt voor jullie niet aflatende belangstelling naar mijn vorderingen met dit boekje. Het heeft mij meer gesterkt dan jullie zullen vermoeden.

Ellen, Jana en Corneel: bedankt voor wie jullie voor mij waren, voor wie jullie zijn, en voor wie jullie zullen zijn, I'm gonna love you - till the wheels come off - ob yea. 


\section{Publicaties}

Verhoeven AC, Boers M. Limited bone loss due to corticosteroids; a systematic review of prospective studies in rheumatoid arthritis and other diseases. J Rheumatol 1997; 24: 1495-503.

Boers M, Verhoeven AC, Markusse HM, van de Laar MAFJ, Westhovens R, van Denderen JC, van Zeben D, Dijkmans BAC, Peeters AJ, Jacobs P, van den Brink HR, Schouten HJA, van der Heijde DMFM, Boonen A, van der Linden S. Randomised comparison of combined step-down prednisolone, methotrexate and sulphasalazine with sulphasalazine alone in early rheumatoid arthritis. Lancet 1997; 350: 309-18.

Boers M, Verhoeven AC, van der Linden S. Combinatietherapie bij vroege reumatoïde artritis: het COBRA-onderzoek. Ned Tijdschr Geneeskd 1997; 141: 2428-32.

Verhoeven AC, Boers M, Tugwell P. Combination therapy in rheumatoid arthritis: up-dated systematic review. Br J Rheumatol 1998; 37: 612-9.

Verhoeven AC, Bibo JC, Boers M, Engel GL, and van der Linden S for the COBRA trial group. Cost-effectiveness and cost-utility of combination therapy in early rheumatoid arthritis:

randomized comparison of combined step-down prednisolone, methotrexate and sulphasalazine with sulphasalazine alone. Br J Rheumatol 1998; 37: 1102-9.

Giltay EJ, Verhoeven AC, van Schaardenburg D, Popp-Snijders C, Boers M, Gooren LJ, Dijkmans BAC. Serum dehydroepiandrosterone sulphate levels in patients with early rheumatoid arthritis: positive association with $\mathrm{G}$-reactive protein, but not with HLA-DR genotype. $\mathrm{Br} J$ Rheumatol 1998; 37: 1254-6.

Pham B, Cranney A, Boers M, Verhoeven AC, Wells G, Tugwell P. Validity of area-under-thecurve analysis to summarize effect in rheumatoid arthritis clinical trials. J Rheumatol 1999; 26: 712-6.

Lassere M, Boers M, van der Heijde D, Boonen A, Edmonds J, Saudan A, Verhoeven AC. Smallest detectable difference in radiological progression. J Rheumatol 1999; 26: 731-9.

Anderson JJ, Wells G, Verhoeven AC, Felson DT. Factors predicting response to treatment in rheumatoid arthritis: the importance of disease duration. A rthritis Rheum 2000; 43: 22-9.

Verhoeven AC, Boers M, van der Linden S. Responsiveness of the core-set, response criteria and utility measures in rheumatoid arthritis. A nn Rheum Dis 2000; 59: 966-74.

Verhoeven AC, Boers M, van der Linden S. Validity of the MACTAR questionnaire as functional index in a rheumatoid arthritis clinical trial. J Rheumatol 2000; 27: 2801-9.

Verhoeven AC, Boers M, te Koppele JM, van der Laan WH, de Roos J, van der Linden S. Reliability of spot samples for assessment of urinary excretion of pyridinoline in patients with rheumatoid arthritis. Clin Exp Rheum 2001; 19: 82-4. 
Lassere MN, van der Heijde D, Johnson K, Bruynesteyn K, Molenaar E, Boonen A, Verhoeven A, Emery P, Boers M. Robustness and generalizability of smallest detectable difference in radiological progression. J Rheumatol 2001; 28: 911-3.

Boers M, Verhoeven AC, van der Linden S. American College of Rheumatology criteria for improvement in rheumatoid arthritis should only be calculated from scores that decrease on improvement. A rthritis Rheum 2001; 44: 1052-5.

Boers M, Kostense PJ, Verhoeven AC, van der Linden S. In early rheumatoid arthritis, inflammation and damage in an individual joint predict further damage in that joint. A rthritis Rheum 2001, 44: 2242-6.

Verhoeven AC, Boers M, te Koppele JM, van der Laan WH, Markusse HM, Geusens P, van der Linden S. Bone turnover, joint-damage and bone mineral density in early rheumatoid arthritis treated with combination therapy including high-dose corticosteroids. Rheumatology 2001, 40: $1231-7$.

Verhoeven AC, Boers M. Behandeling van reumatoïde artritis door blokkade van tumornecrosisfactor met infliximab of etanercept. (Ingezonden). Ned Tijdschr Geneeskd 2001; 145: 2294-5.

Landewé RBM, Boers M, Verhoeven AC, Westhovens R, van de Laar MAFJ, Markusse HM, van Denderen JC, Westedt ML, Peeters AJ, Dijkmans BAC, Jacobs P, Boonen A, van der Heijde DMFM, van der Linden S. COBRA combination therapy in patients with early rheumatoid arthritis: long-term structural benefits of a brief intervention. A rthritis Rheum 2002, 45: 347-56.

Lard LR, Boers M, Verhoeven AC, Vos K, Visser H, Hazes JMW, Z winderman AH, Schreuder GMT, Breedveld FC, de Vries RRP, van der Linden S, Zanelli E, Huizinga TWJ. Early and aggressive treatment of rheumatoid arthritis patients affects the association of HLA class II antigens with progression of joint damage $A$ rthritis $R$ heum 2002, 45: in press.

Knijff-Dutmer EAJ, Drossaers-Bakker W, Verhoeven AC, van der Sluijs Veer GJ, Boers M, van der Linden S, van de Laar MA. Rheumatoid factor measured by fluoro-immunoassay: a responsive measure of rheumatoid arthritis disease activity that is associated with joint damage. $A$ nn Rheum Dis 2002; 61: in press.

Bruynesteyn K, van der Heijde D, Boers M, Verhoeven A, Boonen A, van der Linden S for the COBRA-trial group. Contribution of progression of erosive damage in previously eroded joints in early RA trials; COBRA-trial as an example. A rthritis Rheum 2002, in press. 


\section{Curriculum Vitæ}

De auteur van dit proefschrift werd op 20 juli 1961 geboren te Deventer. Na het behalen van het atheneum- $\beta$ diploma in 1980 aan het Geert Groote College te Deventer starte hij met Psychologie aan de Rijksuniversiteit Groningen. In 1981 begon hij aldaar met Geneeskunde.

$\mathrm{Na}$ student-assistentschappen bij Sociale en Organisatie Psychologie en Interne Geneeskunde en co-schappen op Guraçao ontving hij het arts-diploma in 1990. Na aanstellingen bij de Stichting Nieuw Hoog-Hullen en bij Interne Geneeskunde/Reumatologie in het Academisch Ziekenhuis Groningen, was hij van oktober 1992 tot en met december 1996 verbonden aan de werkgroep Reumatologie (hoofd: prof. dr Sjef van der Linden) in het Academisch Ziekenhuis Maastricht en betrokken bij het door prof. dr Maarten Boers geïnitieerde COBRA-onderzoek (COmbinatietherapie $\mathrm{Bij}$ vroege Reumatoïde Artritis).

$\mathrm{Na}$ een dienstverband als arts-assistent bij Interne Geneeskunde in het Sint Antonius Ziekenhuis te Nieuwegein, werkt hij sinds 1999 als arts voor arbeid en gezondheid bij ArboUnie Oost-Nederland. Sinds 2001 is hij tevens als wetenschappelijk medewerker verbonden aan het Nederlands Kenniscentrum voor Arbeid en Klachten Bewegingsapparaat bij het Erasmus MC in Rotterdam.

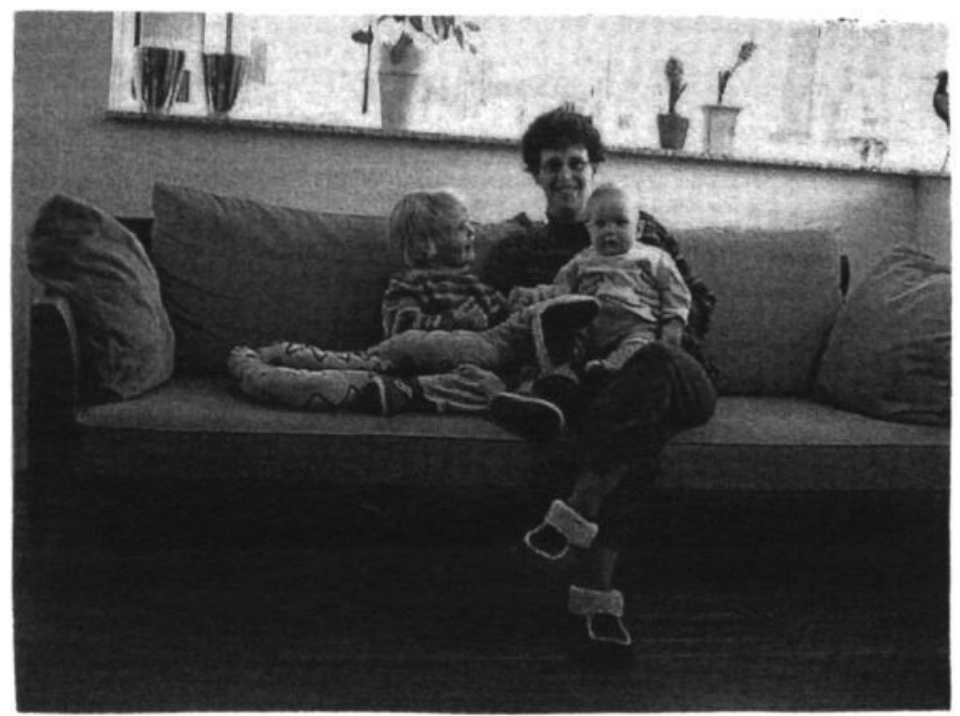


